\title{
Targeting Drug Chemo-Resistance in Cancer Using Natural Products
}

\author{
Wamidh H. Talib *(D), Ahmad Riyad Alsayed, Muna Barakat (D), May Ibrahim Abu-Taha (D) \\ and Asma Ismail Mahmod (iD
}

Department of Clinical Pharmacy and Therapeutic, Applied Science Private University, Amman 11931-166, Jordan; a_alsayed@asu.edu.jo (A.R.A.);m_barakat@asu.edu.jo (M.B.); m_abutaha@asu.edu.jo (M.I.A.-T.); asmamahmod1212@gmail.com (A.I.M.)

* Correspondence: w_talib@asu.edu.jo

Citation: Talib, W.H.; Alsayed, A.R.; Barakat, M.; Abu-Taha, M.I.;

Mahmod, A.I. Targeting Drug Chemo-Resistance in Cancer Using Natural Products. Biomedicines 2021, 9, 1353. https://doi.org/10.3390/ biomedicines 9101353

Academic Editors: Beata Pajak and Anna Jaśkiewicz

Received: 21 August 2021

Accepted: 23 September 2021

Published: 29 September 2021

Publisher's Note: MDPI stays neutral with regard to jurisdictional claims in published maps and institutional affiliations.

Copyright: (C) 2021 by the authors Licensee MDPI, Basel, Switzerland. This article is an open access article distributed under the terms and conditions of the Creative Commons Attribution (CC BY) license (https:// creativecommons.org/licenses/by/ $4.0 /)$.

\begin{abstract}
Cancer is one of the leading causes of death globally. The development of drug resistance is the main contributor to cancer-related mortality. Cancer cells exploit multiple mechanisms to reduce the therapeutic effects of anticancer drugs, thereby causing chemotherapy failure. Natural products are accessible, inexpensive, and less toxic sources of chemotherapeutic agents. Additionally, they have multiple mechanisms of action to inhibit various targets involved in the development of drug resistance. In this review, we have summarized the basic research and clinical applications of natural products as possible inhibitors for drug resistance in cancer. The molecular targets and the mechanisms of action of each natural product are also explained. Diverse drug resistance biomarkers were sensitive to natural products. P-glycoprotein and breast cancer resistance protein can be targeted by a large number of natural products. On the other hand, protein kinase $C$ and topoisomerases were less sensitive to most of the studied natural products. The studies discussed in this review will provide a solid ground for scientists to explore the possible use of natural products in combination anticancer therapies to overcome drug resistance by targeting multiple drug resistance mechanisms.
\end{abstract}

Keywords: anticancer natural products; drug efflux; drug detoxification; plants derived natural products

\section{Introduction}

Cancer is the second most common cause of death after cardiovascular diseases. Statistics from the USA showed that the number of people diagnosed with cancer was 1.7 million in 2017 with 0.6 million deaths [1]. Ninety percent of deaths from cancer result from the development of drug resistance, which leads to the ineffectiveness of chemotherapeutic agents [2]. Drug resistance can be defined as the ability of cancer cells to reduce the potency and efficacy of chemotherapeutic agents [3]. In some types of cancer such as renal cancer and hepatocellular carcinoma, malignant cells start resistance without previous exposure to chemotherapeutic agents (intrinsic resistance) resulting in a poor response to initial treatment [4]. In other cases, cancer cells exhibit initial sensitivity to chemotherapy followed by a poor response due to the development of resistance (aquired resistance) [3]. Previous studies on cell lines and animal models revealed that drug resistance in cancer can be achieved by complex mechanisms, including drug efflux using ATP-binding cassette (ABC) transporter [5], altering the expression of proteins targeted by anticancer drugs [6], drug detoxification [7], augmenting repair mechanisms in DNA [8], and evasion of apoptosis [1]. The use of natural products in the treatment of diseases is very old. Historical documents show that the first use of natural products in medical treatment was reported in Mesopotamia and dates back to 2600 BC [9]. Extensive research was conducted to explore the potential of natural products in cancer therapy. These efforts resulted in the development of some effective drugs derived from natural phytochemicals [10]. Diverse approaches were tested to overcome drug resistance in cancer. However, natural products 
from medicinal plants and other natural sources represent a promising and cost-effective approach [11]. In this review, we summarize natural products that have the potential to overcome drug resistance in cancer. The target of each natural product was identified, and the mechanisms of action were discussed in experimental and clinical studies.

\section{Drug Chemo-Resistance in Cancer: Mechanistic Bases}

Nowadays, one of the most prominent challenges for cancer treatment is drug resistance as malignant cells persuade different mechanisms (Figure 1) to deviate from treatment and maintain their survival. Understanding these mechanisms may facilitate the development of novel drugs with new targeting strategies, which may have a promising clinical implication. In this part of the review, we will discuss several drug-resistance mechanisms that have clinical significance.

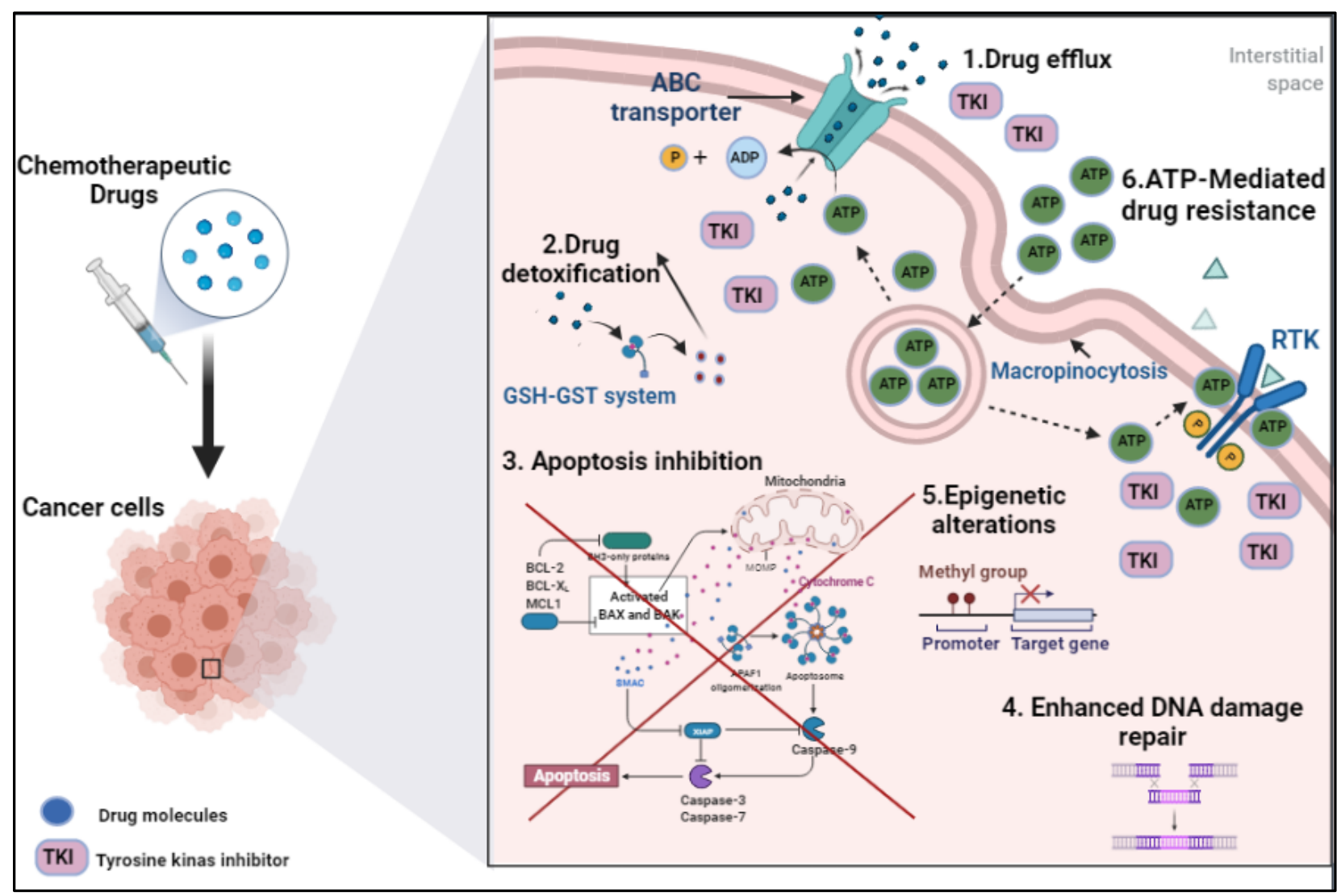

Figure 1. Illustration of drug chemoresistance mechanisms in cancer cells [1]. ABC: ATP binding cassette, RTK: receptor tyrosine kinase, TKI: tyrosine kinase inhibitors.

\subsection{Drug Efflux}

One of the primary mechanisms for chemotherapy resistance is drug efflux, which is defined as drug transportation from the intracellular milieu using energy-dependent pumps [12,13]. High rates of drug efflux in the cancer cells reduce internal drug accumulation and potentiate the cell capability to escape from the treatment [14-16]. This phenomenon could be either intrinsic or acquired; in other words, it either already exists in the cell before or develops post-drug administration [1].

Sophisticated transmembrane transporters direct drug efflux, mainly the ATP-binding cassette ( $\mathrm{ABC}$ ) family [17]. In humans, there are $48 \mathrm{ABC}$ transporters which are stratified into seven subdivisions (ABCA-ABCG) based on phylogenetic analysis $[1,17,18]$. They are involved in the exportation of endogenous substances, e.g., metabolites, vitamins and lipids, in addition to exogenous products such as toxins and drugs [18] Part of these 
transporters play a key role in acquiring multidrug resistance (MDR) characteristics to cancer chemotherapies, such as ABCB1, ABCC1, and ABCG2 [17].

$\mathrm{ABCB} 1$, also known as MDR1 or P-glycoprotein (P-gp), is one of the well-characterized transporters associated with drug resistance for several types of tumors such as leukemia and colorectal, kidney, and lung multiple myeloma $[3,19,20]$. For drug efflux, the cell is coupled with ATP hydrolysis and conformational changes in the transporter [21]. A wide variety of drugs can bind/be pumped through this transporter, such as vincristine, vinblastine, daunorubicin, doxorubicin, epirubicin, etoposide, paclitaxel, mitomycin c, and topotecan [22-24]. Thus, overexpression of ABCB1 potentiates cell competence to hamper the chemotherapy treatment [1].

ABCC1 overexpression, also known as a multidrug resistance-associated protein-1 (MRP1), plays a crucial role in the failure of chemotherapy in a number of malignant tumors, including prostate, breast, and lung cancers [22-25]. ABCC1 transporter can efflux different anticancer drugs such as anthracyclines, camptothecins, vinca alkaloids, a few kinase inhibitors, etoposide irinotecan, and methotrexate [26]. In addition, this type of transporter pumps organic anionic compounds, which are conjugated to either glutathione (GSH), glucuronide, or sulfate $[27,28]$.

ABCG2 is recognized as a primary breast cancer-efflux transporter known as breast cancer resistance protein (BCRP) [29,30]. ABCG2 is expressed in CD133-positive cancer stem cells (CSCs) from human colorectal tumors; accordingly, it is considered a marker for such types of CSCs cancers [31]. Additionally, overexpression of this transporter was notified in other kinds of cancers such as acute myeloid leukemia, endometrial carcinoma, lung cancer, and melanoma $[29,32]$. ABCG2 is capable of translocating a variety of anticancer drugs, including positively and negatively charged drugs, including topoisomerase inhibitors, tyrosine kinase inhibitors, antimetabolites, flavopiridol (cyclin-dependent kinase inhibitor), JNJ-7706621 (CDK and aurora kinases inhibitor), and bicalutamide (non-steroidal anti-androgen) $[29,30]$.

Furthermore, overexpression of $\mathrm{ABCC} 2$ and $\mathrm{ABCC} 3$ has a pivotal effect on the resistance of multiple cytotoxic drugs such as methotrexate, cisplatin, doxorubicin, and etoposide $[33,34]$. These were found to potentiate the drug resistance in breast, liver, and lung cancers [34-36]. Accordingly, a deep understanding of ABC transporters (structure, physiology, overexpression, and mutations) has a promising role in innovating clinically effective anticancer drugs.

\subsection{Drug Detoxification}

Drug detoxification is considered one of the prominent mechanisms to confront chemotherapy treatment. This process involves two main pathways. The first pathway (Phase I) is mediated by cytochrome P450 enzymes (CYP450), which encompasse hydrolysis and oxidation-reduction reactions $[37,38]$. The second pathway (Phase II) comprises primarily conjugation reactions, e.g., glutathionylation, glucuronidation, acetylation, methylation, and sulfonation reactions [39]. This phase is complimentary for the first pathway, as these reactions aim to enhance the hydrophilicity of the parent drug or Phase 1 metabolites in order to be excreted [15]. Moreover, ABC efflux transporters translocate the Phase II conjugated reactions outside the cell [27,28]. For instance, irinotecan (prodrug, topoisomerase- 1 inhibitor) is metabolized in the liver by carboxylesterases to the active 7-ethyl-10-hydroxycamptothecin (SN-38). Then, SN-38 will be exposed to glucuronidation conjugation and active effluxing through $\mathrm{ABC}$ transporters [40]. Therefore, the synergistic activity between the detoxification mechanisms and efflux transporters significantly suppresses the chemotherapeutic effectiveness [41].

One major drug-resistance conjugation pathway is glutathionylation, which is mediated by the GSH-GST system [39]. GST, a family of enzymes that conjugate GSH to the chemo drugs, increased its hydrophilicity and could easily be effluxed out of the cell [27,28]. It has been reported that levels of GSH and GST increase proportionally with the cancer stage; however, there is interindividual variability between patients, which limits its clinical 
implication [42]. On the other hand, a positive correlation was observed between the level of the type GST $\pi$ protein and cancer drug-resistance in variable neoplastic diseases [43-46]. At the gene level, there is a relationship between the GST gene polymorphism and tumor incidence [47] and the efficiency of chemotherapy [48,49].

Unfortunately, several chemotherapeutic drugs are substrates for detoxification processes. Therefore, focusing on the machinery of this area may help in overcoming the resistance problem.

\subsection{Apoptosis Inhibition}

Inhibition of cell death is a fundamental hallmark of cancer. Anticancer medications mainly target this mechanism by inducing programmed cell death, also called apoptosis [50]. Consequently, any alternation in the apoptotic machinery may contribute to drug resistance [15]. Apoptosis occurs through two main pathways: extrinsic and intrinsic [50]. Activation of the extrinsic pathway is mediated by the binding of the tumor necrosis factor family to their receptors on the cell surface, followed by activation of caspase-8, which will promote cell death initiation [51]. Stimulation of the intrinsic pathways is mitochondrially controlled by the imbalance between the pro (e.g., BAX and BAK) and anti-apoptotic proteins (e.g., BCL-2, BCL-XL, BCLw) $[52,53]$. Recruitment of proapoptotic signaling molecules primes the permeabilization of the mitochondrial outer membrane then triggers the release of cytochrome $c$ and leads to activation of series apoptotic reactions via caspases [50].

The disparity between the pro and anti-apoptotic molecules also plays a role in inducing resistance against anticancer therapy [54]. Hence, cancer cells overexpress the anti-apoptotic proteins (such as Bcl-2, Akt, and NF- $\mathrm{kB}$ ) and/or suppress or disturb the production of proapoptotic proteins such as BAX [54-56]. The positive association between the expression level of anti-apoptotic proteins and the ability of cancer cells to evade the treatment has been documented in different types of cancer such as breast cancer, acute myeloid leukemia, and non-Hodgkins lymphoma [57-59]. Presumably, elevated levels of Bcl-2 and Akt hinder cytochrome c release from the mitochondria; thus, the subsequent apoptotic cascade will be discouraged [56]. Activation of Akt is followed by NFKB phosphorylation, which impedes the apoptosis processes and promotes cancer survival. Both Akt and NFKB trigger Bcl-2 inhibitory activity and potentiate the cell resistance power [60]. In the clinical setting, the development of targeted therapy to control the pro or anti-apoptotic proteins may have a promising solution for cancer drug resistance and improve the clinical outcomes.

\subsection{Enhanced DNA Damage Repair}

Numerous chemotherapeutic drugs target DNA damage of the cancerous cells as the main mechanism of action, such as platinum-based drugs, alkylating agents, and anthracyclines [61]. However, this activity may be defended by cancer cell-DNA repair response, which reduces the drug efficacy and potentiates the resistance [62]. Multiple DNA repair mechanisms have been documented in the literature [62,63], including direct reversal, mismatch repair (MMR), nucleotide excision repair (NER), base excision repair (BER), homologous recombination (HR), and nonhomologous end joining (NHEJ). Many factors affect the pathway of the DNA restoration such as tissue location, nature of the DNAdrug adduct, and the involved proteins $[15,64,65]$. For instance, DNA repair endonuclease $\mathrm{XPF}$ and DNA excision repair protein ERCC1 play a vital role in the NER and inter-strand crosslink repair pathways [66]. Studies have shown a positive correlation between the overexpression of these proteins and the establishment of significant drug resistance, e.g., resistance for platinum-based drugs $[67,68]$.

On the other hand, it has been reported that the mortality rate was significantly reduced in patients receiving cisplatin-based chemotherapy with negative-ERCC1 non-small cell lung and breast tumors compared to ERCC1- positive tumors [64,69]. Another example, resistance to the alkylating chemotherapeutic agents, was significantly linked with the overexpression of the $\mathrm{O}_{6}$-methylguanine DNA methyltransferase (MGMT) repair enzyme, 
as glioblastoma patients with increased levels of MGMT showed poor treatment outcomes and higher mortality rates compared with the patients with reduced expression levels [70]. Therefore, such proteins could be a prognostic marker and auspicious therapeutic target for many anticancer drugs.

\subsection{Epigenetic Alterations}

Besides the previous resistance pathways, one of the prominent mechanisms is epigenetic alterations. These alternations mainly affect the function and expression of the cell gene, rather than causing mutations in the DNA sequence [65,71]. Epigenetic alterations could be present in different ways, including DNA methylation patterns, histone modification, chromatin remodeling, and noncoding RNA related alterations $[1,8]$.

DNA methylation is utilized during cell division by adding methyl-group covalently to DNA cytosine through DNA methyltransferases [72]. It has been reported that number of cancer genes are exposed to hypermethylation, which yields transcriptional silencing for the tumor suppressor genes (e.g., CpG promoter islands of tumor suppressor genes) [73,74]. For example, hypermethylation of gene promoters plays a pivotal role in the resistance of ovarian cancer cells towards cisplatin [73]. Conversely, demethylation or hypomethylation was known to affect the chemo-response of cancer cells and upregulate the expression of oncogenes. For instance, hypomethylation of the ABCB1 promoter leads to overexpression of the efflux $A B C B 1$ transporter, which potentiates drug resistance in esophageal squamous cancer cells [75]. Another study has revealed that DNA demethylation and histone modification at the promoter region enhances the overexpression of protein thymosin $\beta 4$ (Tß4), which contributes to drug resistance in hepatocellular carcinoma (HCC) cell line to VEGFR inhibitor sorafenib [76]. A study conducted by Bhatla et al. has demonstrated that suppression of DNA methylation and histone modification in acute lymphoblastic leukemia cells reverse the disease relapse and restore the cell chemosensitivity [77]. Therefore, defeating these resistance mechanisms may have a promising contribution in cancer therapy, as found in the management of resistant-heterogenous multiple myeloma [78].

Moreover, epigenetic modifications could also be present as chromatin remodeling and noncoding RNA-related alterations, including microRNAs (miRNAs) and long noncoding RNAs (lncRNAs) $[79,80]$. MiRNAs modulate the post-transcriptional gene expression and protein synthesis [81]. LncRNAs regulate gene expression through chromatin modification and hinder transcription activation $[79,80]$. Both noncoding RNAs affect the contribution to chemoresistance through modulation of protein production. Various studies have demonstrated overexpression and oncogenic activity of miRNA and lncRNA in different types of cancer such as lymphoma, lung, breast, stomach, colon, and pancreatic cancer [81-83]. Then again, these epigenetic alternations could be considered a futuristic target and have a role in cancer hallmarks.

\subsection{ATP-Mediated Drug Resistance}

Resistance to chemotherapy is also induced by ATP-mediated pathways, either intracellularly or extracellularly. Studies have shown that the intracellular level of ATP in malignant cells is usually more than healthy cells of the same source. In fact, that elevation in intracellular-ATP is mainly caused by increased glycolytic metabolism in a pathway called the Warburg effect [84]. This effect is considered a hallmark in approximately all cancer types $[85,86]$. Additionally, it was reported that drug-resistant cancer cells exhibit higher levels of intracellular ATP than the other tumor cells from the same tissue, which are required for cell survival under cytotoxic conditions [87,88]. For example, a study conducted by Zhou et al. has demonstrated that chemo-resistant colon cancer cell lines express higher levels (i.e., double) of intracellular ATP than non-resistant cells [87]. Contrarywise, sensitivity to chemotherapy was enhanced by diminishing intracellular ATP levels and suppressing the glycolysis process in the resistant cells (i.e., glycolysis, 3-bromopyruvate) [87]. 
Moreover, cancer cells are capable of extensively uptaking the extracellular ATP, subsequently increasing the intracellular ATP, and potentiating the cells' tendency for drug resistance and cancer cell survival [89]. In many cancer types, the extracellular ATP was $10^{3}$ to $10^{4}$ times more than the normal cells from the exact origin [89,90]. Studies have shown that the uptake of extracellular ATP can be utilized mainly through micropinocytosis $[90,91]$. Internalization of ATP to the cancer cell augments the activity of the drug efflux pathway (i.e., through $\mathrm{ABC}$ transporters), which diminishes the intracellular drug concentration and promotes and cancer persistence [89]. In addition, high levels of accumulated intracellular ATP compete with tyrosine kinase inhibitors (TKI) on its receptor (RTK) binding site, which activate phosphorylation and the cascade of cell signaling [92]. Increased ATP internalization promotes TKI translocation (in addition to chemo-drugs) through efflux transporter, which reduces the TKI accumulation inside the cell and increases RTK activity, cell machinery, and resistance [89]. Wang et al. also revealed that drug resistance in the cancer cells mediated by the ability of extracellular ATP molecules to enhance the activity and overexpression of efflux ABC transporters [89]. Shedding light on the blocking/inhibiting mechanisms of extracellular ATP internalization and expression/activity of $\mathrm{ABC}$ transporters might substantially affect the chemosensitivity of tumor cells.

\section{Targets of Natural Products in Cancer Chemo-Resistance}

When a specific cancer type exhibits drug resistance to many drugs, this is referred to as the development of multidrug resistance (MDR) [93]. A potential approach to overcome drug resistance is to target the mechanisms of resistance. The general mechanisms of resistance are currently well recognized; they include increased drug efflux and decreased drug influx, drug inactivation, processing of drug-induced damage, alterations in drug target, and evasion of apoptosis. Certain examples of specific mechanism are the expression of resistant transporters or genes that can enhance drug efflux [94]. Drug efflux, facilitated by membrane transport proteins, is associated with the development of MDR in tumor cells [95]. Overexpression of ATP-binding cassette (ABC) membrane transport proteins has been considered the leading contributor to resistance and chemotherapy failure in several types of cancer [96-98].

An elevated efflux of chemotherapeutic drugs from cancer cells leads to decreased intracellular drug concentrations by pumping drugs out of cells. Drug efflux transporters are mainly responsible for the development of MDR in cancer cells [7,99]. Membrane transport proteins can eliminate drugs from cells and promote drug redistribution. Drug redistribution reduces drug concentrations in the organelles below lethal concentrations, which further reinforces the drug resistance. Some known proteins related to MDR include P-glycoprotein (P-gp), multidrug resistance protein (MRP), breast cancer resistance protein (BCRP), and lung resistance-related protein (LRP) [7].

\subsection{P-Glycoprotein}

P-glycoprotein (P-gp) is also known as multidrug resistance protein-1 (MDR-1), an ABC sub-family-B member-1 encoded in the human body by the ABCB1 gene $[99,100]$. ATP binding causes activation of the ATP-binding domains and the hydrolysis of ATP, which will cause change in the transporter shape, essential for the functioning of the transporter and thus results in drug efflux [99]. P-gp has 12 transmembrane domains and two ATP binding sites on its transporter structure that can be inserted into the cell membrane and bind to a variety of chemotherapeutic drugs. P-gp can detect and bind drugs entering the plasma membrane [101-103]. Its normal function is to protect cells against xenobiotics and cellular toxicants and thus plays an important role in maintaining physiological homeostasis $[104,105]$. P-gp expression varies in various types of cancers. Colon, pancreas, liver, adrenal gland, and kidney cancers demonstrate highest levels of P-gp expression, while intermediate P-gp expression is seen in soft tissue carcinomas, neuroblastoma, and hematological malignancies. Breast, ovary, lung, and esophageal cancers initially display low P-gp levels, but the levels of P-gp efflux transporters in- 
crease after the cancer shows resistance to the chemotherapeutic treatment $[99,101]$. P-gp causes decreased intracellular drug concentration, and overexpression of P-gp is always related to MDR [7,101]. Several P-gp inhibitors generations were developed in hope of circumventing MDR, to block P-gp, and to improve the efficacy of chemotherapy in MDR tumors [102,106-108]. MDR chemosensitizers are P-gp modulators that administered in combination with cytotoxic agents, which are substrates of the efflux pump could restore their efficacy in resistant cancer cells [109].

First-generation drugs appear less potent, non-selective, and have a low P-gp binding affinity. High doses of these inhibitors are required to reverse MDR which can lead to toxic side effects. Second-generation P-gp inhibitors hinder metabolism and excretion of chemotherapeutic drugs by blocking the effects of P-gp. Some shortcomings of secondgeneration P-gp inhibitors, such as interaction with cytochrome P450, were overcome in third-generation P-gp inhibitors [99]. Unfortunately, the first three generations have several safety problems, such as unexpected systemic toxicities, non-targeted inhibition, and unpredictable pharmacokinetic interactions between chemotherapeutic agents and candidate P-gp inhibitors. For these reasons alternative strategies are being pursued by scientists to develop a fourth generation of P-gp inhibitors with safety advantages from natural products $[107,108,110,111]$.

Stemofoline, an alkaloid extracted from Stemona bukilli, was reported to increase the sensitivity of the chemotherapeutic of MDR leukemic cells and raise the accumulation of P-gp substrates (calcein-AM and rhodamine 123). However, it shows no effect in the P-gp expression according to the Western blot analysis [106]. Moreover, Chang et al. have investigated sesquiterpene pyridine alkaloids (wilforine) and their effect on P-gp expression and function. The study shows that wilforine was able to suppress the efflux activity of P-gp in a concentration-related mode along with re-sensitizing MDR cancer cells to chemotherapy agents [112]. Another study has suggested that tenulin and isotenulin, a natural sesquiterpene lactone, have the potential to be improved for synergistic treatment of MDR cancers. It shows significant prevention of the P-gp activity through triggering Pgp ATPase transporter [104]. Moreover, combination of polyphenols such as EGCG, tannic acid, and curcumin exhibited a high synergistic effect with doxorubicin via attenuating the P-gp function in human colon cancer and leukemia cell lines [102]. Moreover, the Western blot analysis shows a reduction in P-gp levels after applying curcumin treatment in K562/DOX cells as well as enhances the sensitivity of the cells to the chemotherapy [113]. Moreover, the expression of P-gp was decreased in A2780/Taxol cells when curcumin and piperine was combined in solid lipid nanoparticle form [114]. Teng et al. suggested that caffeic acid can reduces cancer MDR in human cervical cells (KB/VIN). It inhibited P-gp efflux via attaching to P-gp through GLU74 and TRY117 residues [103]. Recently, quercetin was also reported to have modulation effect on P-gp expression in HeLa and SiHa cells. According to the Western blot analysis, the co-treatment group (quercetin and cisplatin) showed lower levels of P-gp compared to the single-drug groups [107]. Moreover, other studies have shown a quercetin downregulation effect on P-gp efflux function $[108,115,116]$. Kaempferol is a natural flavonoid that was able to reverse the multidrug resistance in HepG2and N1S1 liver cancer cells via reducing P-gp overexpression [117]. Emodin is another natural compound that revealed anticancer activity and improved chemotherapy sensitivity in lung cancer (A549 and H460) via reducing P-gp expression [110]. It also reversed drug resistance and enhanced the sensitivity of cisplatin in A549/DDP cells [111]. Ecteinascidin 74, a marine natural product from Caribbean Sea squirts Ecteinascidia turbinate, can downregulate P-gp expression at a concentration of $0.1 \mathrm{nM}$. In addition, it increased the cellular accumulation of DOX/VCR in P-gp-overexpressed cervix cells [118]. Moreover, using a combination treatment of Sophocarpidine from Sophora flavescens with vincristine and Adriamycin lowered the expression of P-gp in KBV200 cells [7]. Piperine is an alkaloid found in black pepper (Piper nigrum). It has shown downregulation of P-gp, BCRP, MRPs, and $\mathrm{ABC}$ transporter genes (ABCB1, $\mathrm{ABCG} 2$, and $\mathrm{ABCC} 1)$, which may reverse MDR in tumor cells [119-122]. $\beta$-Carotene was also reported to modulate P-gp in resistant cancer 
cell lines (KB-vin and NCI-H460/MX20) and stimulate the basal ATPase activity in a concentration-dependent manner [101,119]. Schisandrin A (Deoxyschizandrin), isolated from Fructus Schizandrae, reversed P-gp-mediated DOX resistance in MCF-7/DOX cells by blocking P-gp, NF-кB, and Stat3 signaling [123]. Moreover, tanshinone microemulsion can significantly reverse drug resistance of K562/ADM cells by inhibiting the P-gp efflux pump effect and increasing the intracellular concentration of chemotherapeutic drugs [124]. Honokiol and magnolol are the main active ingredients in Magnolia officinali. They were able to suppress P-gp in NCI/ADR-RES cells and increase the accumulation of P-gp substrate (calcein) in cells. It was found that magnolol can reverse MDR in U937/ADR cells by inhibiting the activity of NF-KB p65 and by downregulating the expression of MDR1 and P-gp [7,125]. Cepharanthine, coumarins, cycloartanes, didehydrostemofolines, eudesmin, and euphocharacins A-L function as P-gp inhibitors in different cancer cell lines [119]. Other phytochemicals that exhibited an inhibition of P-gp are reported in Table 1.

\subsection{Multidrug Resistance Protein}

Multidrug resistance protein (MRP) belongs to the subfamily $\mathrm{C}$ in the ABC transporter superfamily of cell membrane transporters known to cause MDR. Multidrug resistance associated protein-1 (MRP-1), encoded in the human body by the ABCC2 gene, has been widely studied for its role in developing drug resistance in various cancers. A distinct feature of MRP1 is that it is a basolateral transporter. This implies that MRP1 activity results in the movement of compounds into cells that lie below the basement membrane. The transporter prevents drug absorption from the basolateral side and clears the drugs out of cells [99]. MRP demonstrated a substrate preference for negatively charged drugs and natural products, such as glutathione, glutathione conjugated leukotrienes, glucosylation, conjugation, sulfation, and glucuronylation [102,103,116,125]. This implies that the mechanism of transport of MRP1 is different from that of P-gp transport [99].

MRP1 is expressed ubiquitously in most of the body, including lung, testis, skeletal, and cardiac muscles. Thus, it is present in most of the tumors, including breast cancer, and plays an important role in MDR. Resistance due to MRP / ABCC members (MRPs 1-3) is often caused by an increased efflux and leads to decreased intracellular accumulation of anticancer drugs. Drug targeting of MRP transporters can help to overcome resistance associated with breast cancer cells $[99,100]$. The importance of MRPs in cancer therapy is also implied by their clinical insights. Modulating the function of MRPs to re-sensitize chemotherapeutic agents in cancer therapy shows great promise in cancer therapy; thus, multiple MRP inhibitors have been developed recently [126]. Inhibitors of MRP1 are useful to reverse or prevent acquired drug resistance and to sensitize drug-naïve untreated tumors to anticancer drugs [100]. Various natural products exhibit inhibition activity toward MRPs efflux function. Resveratrol, a polyphenol compound, has downregulated p-gp and MRP1 expression in multidrug-resistant human colon cancer (Caco-2) and CE/ ADR5000 cells. Moreover, it enhanced doxorubicin cytotoxic activity and increased cell sensitivity to chemotherapy [127]. Moreover, three doxorubicin-resistant cell lines of acute myeloid leukemia were treated with resveratrol, and the results exhibited inhibition of cell growth, a significant reduction in MRP1 expression, and an increased uptake of the MRP1 substrate into the cells [128]. Emodin is a natural compound that belongs to the anthraquinone family [129]. It shows anticancer activity and modulation of chemo-resistance of human bladder cancer cells to cisplatin repressing MRP1 [130]. Recently, Guo et al. demonstrated the effect of emodin on gemcitabine resistance in pancreatic cancer cells. The drug resistance associated proteins have been evaluated in PANC-1 cell xenograft in mice. It was revealed that emodin was able to suppress P-gp, MRP1, and MRP5 expression compared to the control group [131]. On the other hand, treating breast cancer cells resistant to tamoxifen with curcumin caused an enhancement in the sensitivity of cells to the chemotherapy mediated by inhibition of the MDR proteins, particularly MRP2 [132]. Moreover, curcumin was also able to reverse cisplatin chemo-resistance in cervical cancer cells via downregulation of MRP1 and P-gp1expression [133]. Quercetin is a natural polyphenol that has 
variety of pharmacological activities including the modulation of efflux transporters. It prevented the accumulation of P-gp, BCRP, and MRP2 in triple negative breast cancer cell lines (MDA-MB-231) [134]. Moreover, epigallocatechin-3 gallate (EGCG), a polyphenolic catechin, showed an impact on chemotherapy resistance mediated by the suppression of MDR-related proteins [135,136]. 7,3', $4^{\prime}$-trihydroxyisoflavone (THIF) is the major metabolite of daidzein. It downregulates the MDR1 promoter region and negatively modulates the MDR1 by controlling transcription factors and then generating new MDR. When THIF is combined with adriamycin, the mRNA expression of MRP, MDR1, and MRP2 was lower than that of adriamycin alone [7]. Moreover, strychnine was found to decrease the gene and protein expression of MRP, but not affect the expression of MDR1 [7]. Some other natural products are mentioned in Table 1.

\subsection{Breast Cancer Resistance Protein}

Breast cancer resistance protein (BCRP), encoded in the human body by the ABCG2 gene, was first identified in a drug-resistant human breast cancer cell line. BCRP belongs to the $\mathrm{ABCG}$ subfamily of $\mathrm{ABC}$ transporters. $\mathrm{BCRP}$ is a half-transporter and dimerization is essential to be functional $[99,101]$. $\mathrm{BCRP}$ has one adenosine $5^{\prime}$-triphosphate $\mathrm{ABC}$ and six transmembrane domains and is, therefore, a so-called half-ABC transporter; BCRP is likely to form a homodimer or homooligomer in order to obtain functional activity $[137,138]$. BCRP is mainly expressed in the cell membranes of multiple organs, including the gastrointestinal tract, liver, kidney, brain, endothelium, mammary tissue, testis, and placenta $[125,139]$. BCRP plays an important role in intercellular drug absorption, metabolism, and excretion, as well as toxicity [99]. BCRP has been extensively studied for its role as an efflux transporter of drugs, leading to drug resistance in target cells and decreased pharmacological effects of substrate drugs. Overexpression of BCRP has been regarded as one of the causes of MDR in different diseases [139]. It causes MDR in most of the types of cancers. In addition to cell membranes, BCRP is also expressed in intracellular vesicles. These vesicles generally retain drugs, but BCRP pumps the drugs out quickly [99]. $\mathrm{BCRP}$ actively extrudes a board range of endogenous and exogenous substrates across biological membranes, which include sulfate conjugates, taxanes, carcinogens, glutamated folates, and porphyrins [125]. This is another reason for increased drug resistance due to BCRP efflux transporter. BCRP is highly expressed in side-population cells in breast cancer [99]. A strong correlation between high ABCG2 expression and poor prognosis of leukemia patients has been described [140]. These cells possess stem cell-like properties and are mostly resistant to chemotherapy. BCRP/ABCG2 inhibitors can have additional benefits besides counteracting MDR [99]. Estrogens and antiestrogens have been shown to reverse cancer drug resistance mediated by BCRP [141]. Among many inhibitors, the most promising ones are bivalent flavonoids, which have shown broad-spectrum inhibitory activity as compared to other classes of compounds [99]. Unfortunately, few clinically useful inhibitors of BCRP have been developed. Therefore, a new, specific BCRP inhibitor is still needed to improve outcomes of drug treatment $[138,139]$. Harmine is a harmala alkaloid that has been used in folk medicine for anticancer therapy [142]. It reversed the resistance of methotrexate and cisplatin drugs in a cancer cell line with BCRP-mediated efflux with no effect on p-gp [132,133,138]. Acacetin, a flavonoid compound, was also reported to have strong reversing activity of BCRP-mediated drug resistance [119,125]. Moreover, apigenin increased the accumulation and inhibition of BCRP in combination with other flavonoids (biochanin A and chrysin) [138,143-145]. Biochanin A is an isoflavone found in red clover with antimutagenesis activity. It inhibited MDR-associated proteins including p-gp, MRP1, and BCRP $[132,133,138,143]$. Other flavonoids such as diosmetin, genistein, kaempferol, luteolin, naringenin-7-glucoside, and quercetin were also reported to have an inhibition effect on BCRP [125]. Recent studies suggested that tangeretin, a natural polymehoxyflavone, showed a potent inhibitory effect on BCRP along with significant suppression of other MDR markers [132,138,143,146]. Terpenoids, hesperetin, rotenoids, 
stilbenoids, daizein, and other phytochemicals that showed an impact on BCRP arelisted in Table 1.

\subsection{Lung Resistance Protein}

Lung resistance protein (LRP) is another transmembrane protein, which is encoded by the LRP gene [99]. It is also known as major vault protein (MVP or VAULT1); vaults are localized in nuclear pore complexes and are involved in nucleocytoplasmic transport and participate in compound transportation in the nucleoplasm $[147,148]$. It was first discovered in non-small cell lung cancer cell line SW-1573. The protein is found in the cytoplasm and in the nuclear membrane of tumor cells. It is not a member of the ABC superfamily of transporter proteins [99]. These vaults may play a role in MDR by regulating the nucleo-cytoplasmic movement of drugs. LRP protein is overexpressed in most cancers, which results in lower accumulation of anticancer drugs in the nucleus. LRP also causes resistance to compounds including alkaloids, anthracyclines, and epipodophyllotoxin. In addition to this, LRP also causes resistance to many drugs, which include doxorubicin (Dox), vincristine, cisplatin, and carboplatin [102,113,149]. In contrast to MRP and P-gp, the transmembrane transport region of LRP lacks the ATP-binding site specific to ABC transporters. This region is not associated with the cell membrane but with transport between the nucleus and cytoplasm [124]. Although the function of LRP is still not fully understood, its role in the formation of barrel-shaped vault organelles is recognized. Vaults transport different molecules between nucleus and cytoplasm. LRP is normally expressed in bone marrow. Positive or higher expression has been associated with adverse outcomes in leukemia as well as multiple solid tumors [148]. Many natural products have overcome chemotherapeutic resistance by downregulation of the lung resistance protein. Ginsenoside $\mathrm{Rg} 3$ is one of the main ginsenosides derived from ginseng. It effectively prevents tumor cell growth in animal models and cell lines as well as targets the MDR factors in resistant cells such as MDR1, MRP, and LRP [144,150,151]. Moreover, peimine, an alkaloid derived from Fritillaria, was able to reverse the MDR of A549/DDP cell line via suppression of ERCC1 mRNA and LRP expression [152]. Paeonol is another natural compound that mediates the inhibition of LRP, P-gp, MDR1, and MRP in multidrug resistance cells [7]. In gastric cancer patients, the expression level of LRP and MDR1 has been blocked following treatment with Chinese herbal medicine (Shen-qi-jian-wei Tang) [94].

\subsection{Protein Kinase C}

Protein kinase C (PKC) is a phospholipid-dependent, cytoplasmic, serine/threonine kinase with a family composed of at least 12 isozymes $[153,154]$. These isozymes are classified into three main groups: classical or conventional PKCs (cPKCs; PKC $\alpha$, PKC $\beta I$, PKX $\beta$ II and PKC $\gamma$ ), novel PKCs (nPKCs; PKC $\sigma, P K C \delta, P K C \varepsilon, P K C \eta$, and PKC $\theta$ ), and atypical PKCs (aPKCs; PKC $\zeta$, and PKC $\lambda$ ) $[155,156]$. Moreover, PKC isozymes have various biological activities including receptor desensitization, transcription modulation, immune signaling regulation, cell growth control, as well as learning memory [155]. In terms of cancer biology, PKC isozymes mediate different signal transduction of cell proliferation, differentiation, angiogenesis, and programmed cell death [157-159]. Tumorigenesis and drug resistance are associated with the interruption of protein kinase $C$ regulation [153]. Several preclinical studies have shown the effect of blocking PKC on drug resistance and the enhancement of the conventional chemotherapy cytotoxic activity [158-160]. Moreover, upregulation of PKC expression in the cytosolic and nuclear compartments was reported in particular MDR tumor cell lines compared to the parent cells [161-164]. Many phosphorylation reactions and binding of cofactors are controlling the activity of PKC [143]. PKC isozymes may be activated by $\mathrm{Ca}^{+2}$, diacylglycerol (DAG), and phospholipids [165]. Moreover, phorbol ester, a tumor promoter, is also able to activate PKCs as it mimics the action of DAG [144]. In MDR cancer cell lines, a study found a correlation between high PKC transduction signaling, particularly $\mathrm{CPKC}$ and $\mathrm{nPKC}$, and the upregulation of P-glycoprotein phosphorylation as well as induction of intracellular MDR phenotypes [158,166,167]. Plant-derived products 
showed great potential to reverse MDR in cancer cells through different mechanisms as inhibiting PKCs is one of these pathways [7]. Curcumin, a polyphenolic compound, was able to suppress the expression of PKC- $\alpha$ and $-\zeta$ in breast cancer cell lines (MCF-7 and MDA-MB-231), resulting in sensitizing tumor cells to the chemotherapeutic drugs [145]. Flavonoids such as quercetin also showed an inhibition effect on PKC transduction in hepatocellular carcinoma [146]. Due to the PKC isozyme's complex role in the cellular functions, inhibition or stimulation of these isoforms might lead to reducing multidrug resistance in cancer cells [168]. Russo et al. found that quercetin mediated CD95-resistant cell line apoptosis via activation of PKC $\alpha$ [169]. The effect of phorbol esters and other diterpenoids on PKCs has been reviewed extensively by Remy and Litaudon (1), demonstrating phorbols interaction with PKCs based on substitution pattern high potency. This interaction involves hydrogen bonding and hydrophobic links, which end up with complex formation with PKCs and reduce their activity [149-151]. On the other hand, tigliane diterpenoids are essential derivatives of the Euphorbiaceae family [170], particularly prostratin (a 12-deoxyphorbol ester), which has been reported to be a potent stimulator of PKCs without pro-tumoral activity [157]. Prostratin has potent antiviral and anticancer activities, especially against liver, breast, and gastric tumors [158].

\subsection{Glutathione Transferase}

Glutathione transferases (GSTs) are multifunctional enzymes known as phase II metabolic enzymes that function as cellular detoxifying agents. They can break down the glutathione part of non-polar xenobiotics and endogenous molecules converting them to more water-soluble compounds to ease their removal $[159,171,172]$. The GST family consists of different isozymes classes including $\alpha, \Sigma, Z, \Omega \mu, \pi$, and $\theta$, which are responsible for catalyzing a wide range of substances $[160,173]$. Moreover, it was found that a high level of GSTs are associated with developing MDR in cancer cells $[61,174,175]$. The antioxidant activity of GSTs mediates the chemo-resistance in tumor cells via detoxifying the anticancer drugs and, as a result, reducing cells' sensitivity to the treatment $[173,176]$. Several studies have shown the correlation between GST overexpression and chemo-resistance in various types of cancer, such as lung cancer [177-179], breast cancer [166,167,180], brain [181,182], and gastric cancer $[183,184]$. Thus, many synthesized and natural GST inhibitors have been investigated to control the multidrug resistance in cancer cells [159]. Curcumin has known for its antioxidant, anti-inflammatory, and chemopreventive activity $[173,185]$. It shows an impact on MDR markers by inhibiting GST $\pi$ in the non-small cell lung carcinoma cell line (NCI-H460/R) [186]. Moreover, it reduced drug resistance in melanoma cells by downregulation of GST and MRP1 [187]. Emodin is a type of natural anthraquinone presented in much herbal medicine [188]. It exhibited a reversal effect on multidrug-resistant promyelocytic leukemia (HL-60/ADR cells) and human oral squamous carcinoma (KBV200 cells) via many pathways' one of them was the reduction of GST $\pi[189,190]$. Majidinia et al. have also reported the suppression effect of emodin and quercetin on GST $\pi$ to overcome MDR in cancer cells [191]. Moreover, fisetin, a plant flavonol, was significantly able to downregulate GST expression in colorectal adenocarcinoma cells (Caco-2), which made fisetin a promising GST-targeted chemosensitizer for modulating MDR [192]. Yu Ping Feng San (YPFS) is a popular Chinese herbal combination formula composed of Astragali Radix, Atractylodis Macrocephalea Rhizoma, and Saposhnikoviae Radix. Du et al. have tested the activity of (YPFS) on cisplatin-resistant lung cancer (A549/DDP cells). It reduced MDR-associated proteins and enzymes such as ATP-binding cassette transporters and GSTs isozymes [178]. Another mixture of Chinese supplement energy and nourish lung (SENL) herbs consisting of ginsenoside Rg1, ginsenoside Rb1, ginsenoside Rg3, astragaloside IV, ophiopogonin D, and tetrandrine has been investigated in A549/DDP cells. It reduced the GST $\pi$ expression and reversed the cisplatin resistance in lung cancer xenografts [193]. Furthermore, ginger phytochemicals (6-gingerol, 10-gingerol, 4-shogaol, 6-shogaol, 10-shogaol, and 6-dehydrogingerdione) inhibited GST $\pi$ and MRP1 in docetaxel-resistant prostate cancer (PC3R) [194]. Another study suggested that oridonin, a tetracyclic diterpenoid extracted 
from Rabdosia labtea, stimulated the apoptosis-associated markers in gemcitabine-resistant PANC-1 pancreatic cancer cells. It suppressed the expression of GST $\pi$ and lipoprotein receptor protein 1 (LRP1) [195]. Natural phenols such as resveratrol have shown modulation of multidrug resistance in tumor cells. Treating doxorubicin-resistant Caco-2 cells with resveratrol revealed a significant reduction in GST mRNA levels along with various MDR markers [127]. Moreover, dietary carotenoids particularly fucoxanthin (FUC), a nonpro-vitamin A carotenoid found in brown seaweeds, have displayed antioxidant potential and improved many cancer cells' sensitivity toward chemotherapies [196,197]. Eid et al. demonstrated the effect of FUC on enhancing doxorubicin activity and mediated apoptosis via increasing caspases and p53 as well as downregulation of GST, CYP3A4, and PXR in resistant cancer cells [166].

\subsection{Topoisomerases}

DNA topoisomerases (topo) are enzymes found in the nucleus of cells. They regulate DNA replication, repair, and chromosomal segregation by converting DNA topology [198]. There are two kinds of topoisomerases: topo I and II, with different classes implementing various functions. Topo I catalyzes the breaking of single strands of DNA, while topo II cutting the double strands of DNA to relieve the supercoiling $[199,200]$. Cell-cycle arrest and cell death by apoptosis are the results of blocking one type of topoisomerase, while blocking the two types can highly improve the cytotoxicity toward cancer cells [201,202]. Many cancer cells have shown a high level of topo II expression, which makes it a target for new chemotherapy [203]. Topo II has two main isoforms: topo II $\alpha$ and topo II $\beta[204,205]$. Since topo II $\alpha$ has an important role in cell growth, it is highly expressed in fast-growing cancer cells. On the other hand, topo II $\beta$ is present in dormant cells in all kinds of tissues during the whole cell cycle $[205,206]$. Many powerful chemotherapy drugs such as doxorubicin, teniposide, and etoposide are topoisomerase II inhibitors [205]. However, serious side effects could result from using these drugs due to the lack of selectivity as well as the risk of drug resistance due to the enzymes' gene mutation or dysregulation of their expression in tumor cells [194,207-209]. Thus, looking for new phytochemicals that targeting topoisomerases enzyme is a promising branch in chemotherapy development. Many secondary metabolites have an impact on topoisomerase enzymes such as alkaloids, flavonoids, and triterpenes [201,210-213]. Emodin is an example of a natural product that reversed the multidrug resistance in promyelocytic leukemia (HL-60/ADR cells). It reduced the expression of MDR proteins including topo II $\beta$ and MRP1 along with increasing the intracellular accumulation of adriamycin (ADR) and daunorubicin (DNR) [189]. This effect was also reported in resistant human oral squamous carcinoma cells [190]. Moreover, curcumin was able to downregulate the topo II $\alpha$ in human non-small cell lung carcinoma cells (NCI-H460/R cells) [186]. Riccardin D is a macrocyclic bisbibenzyl extracted from the Chinese liverwort plant. It promoted apoptosis and reduce MDR in leukemia cells via inhibition of topoisomerase II and decreasing p-gp expression [214].

\subsection{Hypoxia-Inducible Factor}

Hypoxia usually developed in rapidly growing cancer cells. It is a major problem in achieving effective cancer chemotherapy $[215,216]$. Tumor hypoxia has been known to stimulate the expression of several genes correlated with drug resistance [217]. Hypoxiainducible factor- 1 is an oxygen-sensitive heterodimeric transcription factor composed of two subunits: $\alpha$ and $\beta$ [217-219]. It was reported that chemoresistance is associated with a high level of HIF- $1 \alpha$ expression in many cancer types including ovarian cancer, hepatocellular carcinoma, glioblastoma, and colorectal cancer [220-223]. Moreover, HIF-1 $\alpha$ can trigger more than 60 genes involved in tumor growth, metastasis, cellular metabolism, the reduction of apoptosis, and poor prognosis [224,225]. HIF- $1 \alpha$ follows different pathways to promote tumor drug resistance, and one of them is by regulating MDR-associated proteins such as p-gp and MRP1 [226,227]. Natural products and their derivatives are an abundant source of safe and effective resistance reversal agents [228]. Epigallocatechin-3-gallate 
(EGCG) is a polyphenol extracted from green tea and one of the MDR reversal modulators $[228,229]$. Wen et al. suggested that an EGCG derivative reduced drug resistance in doxorubicin-resistant human hepatocellular carcinoma cells (BEL-7404/DOX) via downregulation of HIF- $1 \alpha$ and p-gp [228]. Moreover, apigenin, a type of flavonoid, reversed paclitaxel resistance in hypoxic-liver tumor cells by inhibiting HIF-1 $\alpha$ [230]. Treating drugresistant prostate carcinoma cells (DU-145 cell line) with emodin improved the efficacy of cisplatin and attenuated MDR markers expression and suppressed HIF-1 $\alpha$ [207,231]. Interestingly, quercetin was able to inhibit HIF-1 $\alpha$ and MDR1 as a result of the enhanced cytotoxic activity of doxorubicin and gemcitabine in pancreatic and liver cancer cells [232]. On the other hand, resveratrol repressed the hypoxia-induced resistance to doxorubicin in MCF-7 cells via downregulation of HIF-1 $\alpha$ protein expression [208]. Nuciferine is an aromatic alkaloid extracted from lotus leaves that exhibited anti-inflammatory, antioxidant, and anticancer properties [209,233,234]. Recently, nuciferine has been applied in drug-resistant tumor cells, and it was able to regulate MDR proteins as well as reduce the activation of Nrf2 and HIF- $1 \alpha$ [235]. Modulation of HIF- $1 \alpha$ by curcumin was also reported $[236,237]$. Figure 2 displays the main natural products and their targets in cancer multidrug resistance.

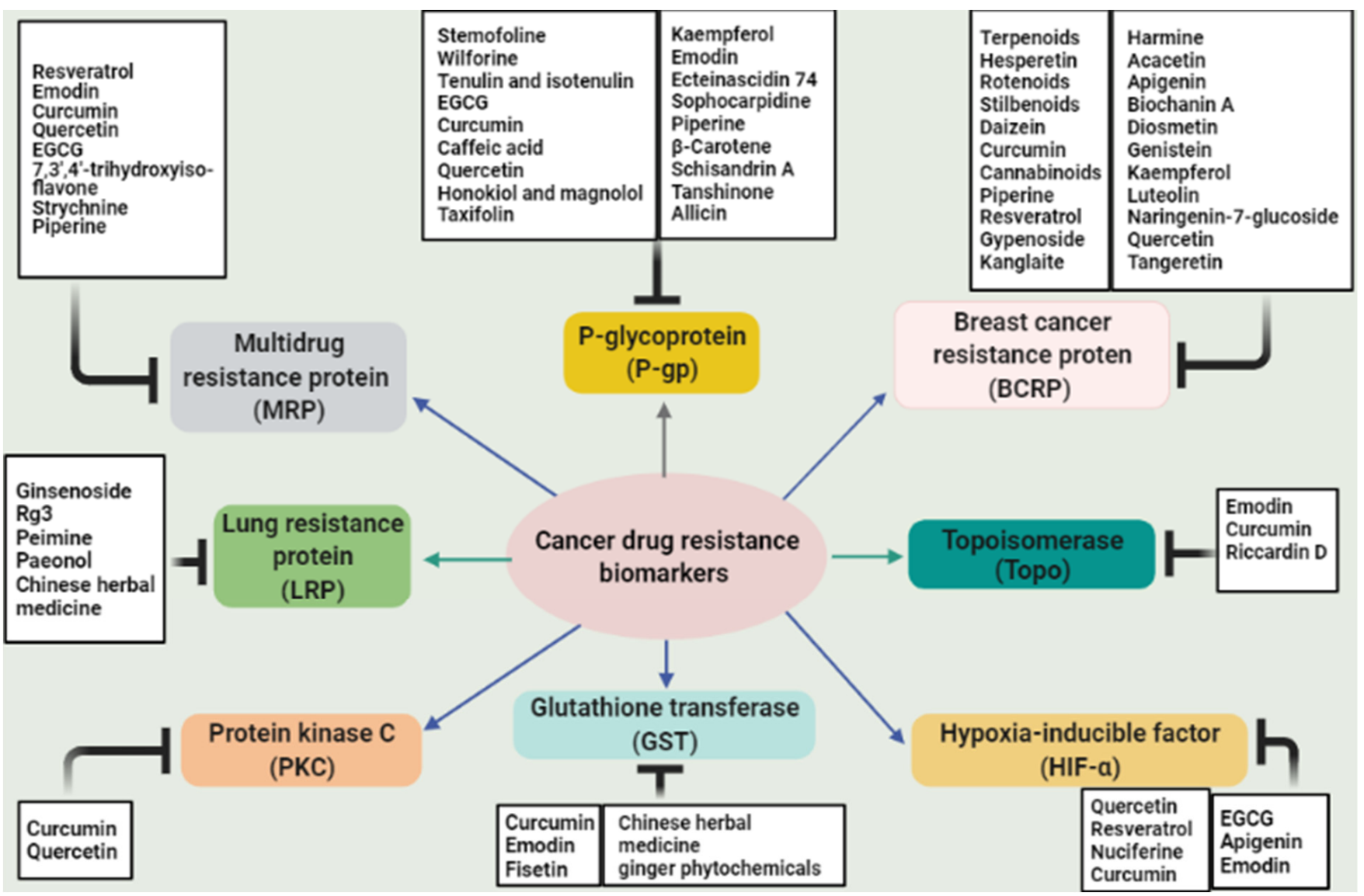

Figure 2. Summary of the main natural compounds targeting multidrug resistance biomarkers in cancer. 
Table 1. Natural products with their mechanisms of inhibition.

\begin{tabular}{|c|c|c|}
\hline Substance & Mechanism of Inhibition & References \\
\hline Dauriporphine & $\downarrow$ P-g expression & [125] \\
\hline Glaucine & $\begin{array}{l}\downarrow \text { P-g expression } \\
\quad \downarrow \text { MDR1 } \\
\quad \downarrow \text { MRP1 }\end{array}$ & [125] \\
\hline Hernandezine & $\downarrow$ P-g expression & [125] \\
\hline Antofine & $\begin{array}{l}\downarrow \text { P-g expression } \\
\downarrow \text { MDR1 mRNA }\end{array}$ & [125] \\
\hline Harmine & $\downarrow \mathrm{BCRP}$ & {$[132,133,138]$} \\
\hline Tryptanthrin & $\begin{array}{c}\downarrow \text { P-g expression } \\
\downarrow \text { MRP2 }\end{array}$ & [125] \\
\hline $\begin{array}{c}\text { Lobeline } \\
\text { (from Lobelia inflate) }\end{array}$ & $\downarrow$ P-g expression & {$[120,125]$} \\
\hline Tetramethylpyrazine & $\begin{array}{c}\downarrow \text { P-g expression } \\
\downarrow \text { MDR1 mRNA } \\
\downarrow \text { MRP1, MRP2, MRP3 }\end{array}$ & {$[105,138,144,238]$} \\
\hline $\begin{array}{l}\text { Danshensu and } \\
\text { tetramethylpyrazine (from the } \\
\text { Chinese herbs) }\end{array}$ & $\downarrow$ P-g expression & [239] \\
\hline Acrimarine E & $\downarrow$ P-g expression & [125] \\
\hline Gravacridonetriol & $\downarrow$ MDR1 mRNA & [125] \\
\hline 2-Methoxycitpressine I & $\downarrow$ P-g expression & [125] \\
\hline $\begin{array}{c}\text { Capsaicin } \\
\text { (extracted from Capsicum } \\
\text { annuum) }\end{array}$ & $\downarrow$ P-g expression & {$[100,125]$} \\
\hline Acacetin & $\begin{array}{l}\downarrow \mathrm{BCRP} \\
\downarrow \mathrm{MRP} 1\end{array}$ & {$[119,125]$} \\
\hline Amorphigenin & $\downarrow$ P-g expression & [125] \\
\hline Apigenin & $\begin{array}{c}\downarrow \text { BCRP } \\
\downarrow \text { MRP1 } \\
\downarrow \text { P-g expression } \\
\downarrow \text { HIF- } 1 \alpha\end{array}$ & {$[7,138,143,144,240]$} \\
\hline Ampelopsin & $\downarrow$ P-g expression & [125] \\
\hline Biochanin A & $\begin{array}{c}\downarrow \text { BCRP } \\
\downarrow \text { MRP1 } \\
\downarrow \text { P-g expression }\end{array}$ & {$[132,133,138,143]$} \\
\hline Catechin & $\begin{array}{l}\downarrow \text { ATPase activity } \\
\downarrow \text { P-g expression }\end{array}$ & [125] \\
\hline Chalcone & $\begin{array}{c}\downarrow \text { MRP1 } \\
\downarrow \text { P-g expression }\end{array}$ & $\begin{array}{l}{[125]} \\
{[141]}\end{array}$ \\
\hline Chrysin & $\begin{array}{c}\downarrow \text { BCRP } \\
\downarrow \text { P-g expression }\end{array}$ & $\begin{array}{c}{[125]} \\
{[119,141]}\end{array}$ \\
\hline Diosmetin & $\downarrow \mathrm{BCRP}$ & {$[125]$} \\
\hline $\begin{array}{l}\text { Green tea catechins (EGCG, } \\
\text { ECG, CG) }\end{array}$ & $\begin{array}{c}\downarrow \text { P-g expression } \\
\downarrow \text { MDR1 } \\
\downarrow \text { ATPase activity }\end{array}$ & {$[100,141]$} \\
\hline Epicatechin gallate & $\downarrow$ P-g expression & {$[118,125]$} \\
\hline
\end{tabular}


Table 1. Cont.

\begin{tabular}{|c|c|c|}
\hline Substance & Mechanism of Inhibition & References \\
\hline Epigallocatechin gallate & $\begin{array}{c}\downarrow \text { P-g expression } \\
\quad \downarrow \text { MDR1 } \\
\quad \downarrow \text { ABCG } 2 \\
\quad \downarrow \text { HIF- } 1 \alpha\end{array}$ & {$[113,114,132,138,143,144,241]$} \\
\hline Formononetin & $\downarrow$ P-g expression & [125] \\
\hline Genistein & $\begin{array}{l}\downarrow \mathrm{BCRP} \\
\downarrow \mathrm{MRP} 1\end{array}$ & {$[132,133,138,143]$} \\
\hline Glabridin & $\downarrow$ P-g expression & {$[7,114,138]$} \\
\hline $\begin{array}{c}3,3^{\prime}, 4^{\prime}, 5,6,7,8- \\
\text { Heptamethoxyflavone }\end{array}$ & $\downarrow$ P-g expression & [125] \\
\hline Kaempferol & $\begin{array}{c}\downarrow \text { BCRP } \\
\downarrow \text { MRP1 } \\
\downarrow \text { P-g expression }\end{array}$ & {$[7,134,138,143]$} \\
\hline Luteolin & $\begin{array}{l}\downarrow \mathrm{BCRP} \\
\downarrow \mathrm{MRP} 1\end{array}$ & {$[125,141]$} \\
\hline Morin & $\begin{array}{c}\downarrow \text { P-g expression } \\
\downarrow \text { MRP1 }\end{array}$ & {$[132,133,138,143]$} \\
\hline Myricetin & $\begin{array}{c}\downarrow \text { MRP1 and MRP2 activity } \\
\downarrow \text { Calcein efflux }\end{array}$ & {$[7,105,133,138,143]$} \\
\hline Naringenin & $\downarrow$ P-g expression & {$[134,138,143]$} \\
\hline Naringenin-7-glucosid & $\downarrow \mathrm{BCRP}$ & [125] \\
\hline $\begin{array}{l}\text { Nobiletin (found in citrus } \\
\text { fruit) }\end{array}$ & $\begin{array}{c}\downarrow \text { P-g expression } \\
\downarrow \text { MRP1 }\end{array}$ & {$[114,138,143,146]$} \\
\hline Phloretin & $\begin{array}{c}\downarrow \text { P-g expression } \\
\downarrow \text { MRP1 }\end{array}$ & {$[132,133,138,143]$} \\
\hline Procyanidine & $\downarrow$ P-g expression & [125] \\
\hline Quercetin & $\begin{array}{c}\downarrow \text { MRP1-mediated drug } \\
\text { transport } \\
\downarrow \text { BCRP } \\
\downarrow \text { MRP1, 4 and 5. } \\
\downarrow \text { P-g expression } \\
\downarrow \text { PKC } \\
\downarrow \text { HIF- } 1 \alpha \\
\downarrow \text { MDR } 1\end{array}$ & $\begin{array}{c}{[7,118-120,124,125,141,146} \\
169,232,242-247]\end{array}$ \\
\hline Robinetin & $\begin{array}{l}\downarrow \text { MRP1 and MRP2 activity } \\
\text { (inhibited calcein efflux) }\end{array}$ & [125] \\
\hline Rotenone & $\downarrow$ P-g expression & [125] \\
\hline Silymarin & $\begin{array}{c}\downarrow \text { P-gp ATPase activity } \\
\downarrow \text { P-gp-mediated cellular } \\
\text { efflux } \\
\downarrow[3 \text { H]azidopine photoaffinity } \\
\text { labeling of P-gp suggesting a } \\
\text { direct interaction with the } \\
\text { P-gp substrate binding } \\
\downarrow \text { MRP1-mediated drug } \\
\text { transport } \\
\downarrow \text { BCRP }\end{array}$ & {$[7,132,133,138,143]$} \\
\hline Tangeretin & $\begin{array}{c}\downarrow \text { P-g expression } \\
\downarrow \text { BCRP }\end{array}$ & {$[132,138,143,146]$} \\
\hline
\end{tabular}


Table 1. Cont.

\begin{tabular}{|c|c|c|}
\hline Substance & Mechanism of Inhibition & References \\
\hline Curcumin & $\begin{array}{c}\downarrow \text { P-g expression } \\
\downarrow \text { BCRP } \\
\downarrow \text { MRP1 } \\
\downarrow \text { MDR1 mRNA } \\
\downarrow \text { ABCG2 and ABCC1 } \\
\downarrow \text { PKC- } \alpha \text { and }-\zeta \\
\downarrow \text { GST } \pi \\
\downarrow \text { Topo II } \alpha \\
\downarrow \text { HIF- } 1 \alpha\end{array}$ & $\begin{array}{c}{[7,94,100,118-120,124,125,145,} \\
186,236,237,242,246,248-251]\end{array}$ \\
\hline $\begin{array}{c}\text { Matairesinol } \\
\text { (found in soybean (Glycine } \\
\text { max)) }\end{array}$ & $\begin{array}{l}\downarrow \text { P-g expression } \\
\downarrow \text { MRP1 }\end{array}$ & {$[100,125]$} \\
\hline Sesamin & $\downarrow$ P-g expression & {$[100,125]$} \\
\hline Gomisin A & $\downarrow$ P-g expression & [125] \\
\hline Schisandrol A & $\downarrow$ P-g expression & $\begin{array}{l}\text { [125] } \\
\text { [119] }\end{array}$ \\
\hline Chlorogenic acid & $\downarrow$ P-gp ATPase activity & [125] \\
\hline Ginkgolic acid & $\begin{array}{l}\uparrow \text { DNR accumulation } \\
\downarrow \text { P-g expression }\end{array}$ & [125] \\
\hline Agnuside & $\downarrow$ P-gp ATPase activity & [125] \\
\hline Picroside-II & $\downarrow$ P-gp ATPase activity & [125] \\
\hline Santonin & $\downarrow$ P-gp ATPase activity & [125] \\
\hline beta-Amyrin & $\downarrow$ P-g expression & [125] \\
\hline $\begin{array}{l}\text { Glycyrrhetinic acid } \\
\text { (Enoxolone) } \\
\text { (Licorice) }\end{array}$ & $\begin{array}{c}\downarrow \text { P-g expression } \\
\downarrow \text { MRP1 }\end{array}$ & {$[100,125]$} \\
\hline Obacunone & $\downarrow$ P-g expression & [125] \\
\hline Oleanolic acid & $\downarrow$ P-g expression & {$[125,247]$} \\
\hline Uvaol & $\downarrow$ P-g expression & {$[125,247]$} \\
\hline Alisol B 23-acetate & $\downarrow$ P-g expression & {$[113,133,138]$} \\
\hline Ginsenoside $\mathrm{Rg}_{3}$ & $\begin{array}{c}\downarrow \text { Binding of }[3 \mathrm{H}] \text { azidopine } \\
\text { to P-gp } \\
\downarrow \text { P-g expression }\end{array}$ & {$[119,125]$} \\
\hline $\begin{array}{c}\text { Protopanaxatriol ginsenosides } \\
\text { 20S-ginsenoside } \\
\text { Ginsenoside } \mathrm{Rb} 1 \\
\text { Ginsenoside Rg3 }\end{array}$ & $\begin{array}{c}\downarrow \text { P-g expression } \\
\quad \downarrow \text { BCRP } \\
\downarrow \text { MRP1 } \\
\quad \downarrow \text { MDR1 } \\
\quad \downarrow \text { LRP }\end{array}$ & {$[113,133,138,144,146]$} \\
\hline $\begin{array}{c}\text { Tenacigenin B: P8, P26 and } \\
\text { P27 }\end{array}$ & $\begin{array}{c}\downarrow \text { P-g expression } \\
\downarrow \text { MRP1 } \\
\downarrow \text { ABCG2-mediated efflux }\end{array}$ & [125] \\
\hline Tenacigenin B: P2, P3 and P6 & $\begin{array}{c}\downarrow \text { P-g expression } \\
\downarrow \text { MRP1 }\end{array}$ & [125] \\
\hline $\begin{array}{c}\text { Tenacigenin B: P1, P4, P5, P9 } \\
\text { and P28 }\end{array}$ & $\downarrow$ P-g expression & [125] \\
\hline Aurochrome & $\downarrow$ P-g expression & [125] \\
\hline Diepoxycarotene & $\downarrow$ P-g expression & [125] \\
\hline Mutatochrome & $\downarrow$ P-g expression & [125] \\
\hline Clausarin & $\downarrow$ P-gp-mediated drug efflux & [125] \\
\hline
\end{tabular}


Table 1. Cont.

\begin{tabular}{|c|c|c|}
\hline Substance & Mechanism of Inhibition & References \\
\hline Phyllodulcin & $\begin{array}{c}\uparrow \text { DNR accumulation } \\
\text { (inhibition of P-gp-mediated } \\
\text { efflux of DNR) }\end{array}$ & [125] \\
\hline Acteoside (Verbascosine) & $\downarrow$ P-gp ATPase activity & [125] \\
\hline Berbamine & $\downarrow$ MDR1 gene expression & {$[125,242]$} \\
\hline Glaucine & $\begin{array}{c}\downarrow \text { P-g expression } \\
\downarrow \text { MRP1 } \\
\downarrow \text { MDR1 and MRP1 genes }\end{array}$ & [125] \\
\hline Fangchinoline & $\downarrow$ P-g expression & [125] \\
\hline $\begin{array}{l}\text { O-(4-ethoxyl-butyl)- } \\
\text { berbamine }\end{array}$ & $\downarrow$ MDR1 gene expression & [125] \\
\hline $\begin{array}{c}\text { Tetrandrine } \\
\text { (dried root of Stephania } \\
\text { tetrandra) }\end{array}$ & $\begin{array}{c}\downarrow \text { P-g expression } \\
\downarrow \text { LRP }\end{array}$ & {$[103,113,135,138,144]$} \\
\hline Matrine & $\downarrow$ P-g expression & [125] \\
\hline Antofine & $\begin{array}{l}\downarrow \text { MDR1 mRNA } \\
\downarrow \text { P-g expression }\end{array}$ & [125] \\
\hline Ephedrine & $\begin{array}{l}\downarrow \text { MDR1 mRNA } \\
\downarrow \text { P-g expression }\end{array}$ & {$[125,242]$} \\
\hline Indole-3-carbinol & $\downarrow$ P-g expression & [125] \\
\hline Staurosporine & $\begin{array}{c}\downarrow \text { P-g expression } \\
\downarrow \text { MDR1 gene expression }\end{array}$ & [125] \\
\hline Vauqueline & $\begin{array}{l}\downarrow \text { MDR1 mRNA } \\
\downarrow \text { P-g expression }\end{array}$ & [125] \\
\hline Gravacridonetriol & $\downarrow$ MDR1 mRNA & [125] \\
\hline Clitocine & $\begin{array}{l}\downarrow \text { MDR1 mRNA } \\
\downarrow \text { P-g expression) }\end{array}$ & [125] \\
\hline Sulfinosine & $\begin{array}{l}\downarrow \text { MDR1 mRNA } \\
\downarrow \text { P-g expression }\end{array}$ & [125] \\
\hline Bisdemethoxycurcumin & $\begin{array}{c}\downarrow \text { P-gp expression } \\
\downarrow \text { MDR1 }\end{array}$ & {$[118,125]$} \\
\hline $\begin{array}{l}\text { Honokiol and magnolol } \\
\text { (isolated from Magnolia } \\
\text { officinali) }\end{array}$ & $\begin{array}{c}\downarrow \text { MDR1 } \\
\downarrow \text { P-gp expression }\end{array}$ & {$[7,125]$} \\
\hline $\begin{array}{c}\text { Schisandrin A } \\
\text { (Deoxyschizandrin) }\end{array}$ & $\begin{array}{c}\downarrow \text { P-gp expression } \\
\downarrow \text { MDR1 } \\
\downarrow \text { PKC }\end{array}$ & {$[104,113,125,133,137,146]$} \\
\hline Schisandrin B (Sch B) & $\begin{array}{c}\downarrow \text { P-gp expression and P-gp } \\
\text { mediated efflux of Dox. } \\
\downarrow \text { MRP1 }\end{array}$ & [93] \\
\hline Triptolide & $\begin{array}{c}\downarrow \text { MDR1 } \\
\downarrow \text { MRP1 protein expression }\end{array}$ & [125] \\
\hline Pyranocoumarins & $\begin{array}{c}\downarrow \text { P-gp expression } \\
\downarrow \text { MDR1 mRNA expression }\end{array}$ & {$[119,125]$} \\
\hline
\end{tabular}


Table 1. Cont.

\begin{tabular}{|c|c|c|}
\hline Substance & Mechanism of Inhibition & References \\
\hline $\begin{array}{l}\text { Ginger phytochemicals } \\
\text { (6-Gingerol,10- Gingerol) }\end{array}$ & $\begin{array}{c}\downarrow \text { P-gp expression } \\
\downarrow \text { MRP1 }\end{array}$ & {$[100,125]$} \\
\hline $\begin{array}{l}\text { Ginger phytochemicals } \\
\text { (6-gingerol, 10-gingerol, } \\
\text { 4-shogaol, 6-shogaol, } \\
\text { 10-shogaol, and } \\
\text { 6-dehydrogingerdione) }\end{array}$ & $\begin{array}{l}\downarrow \mathrm{GST} \pi \\
\downarrow \mathrm{MRP} 1\end{array}$ & [194] \\
\hline Alisma orientalis & $\downarrow$ P-gp expression & [250] \\
\hline Piper methysticum & $\downarrow$ P-gp expression & [250] \\
\hline Guggulsterone & $\begin{array}{c}\downarrow \text { P-gp expression } \\
\downarrow \text { MRPs }\end{array}$ & {$[113,114,134,252]$} \\
\hline Phenolic diterpenes & $\downarrow$ P-gp expression & [250] \\
\hline Vincristine & $\downarrow$ P-gp expression & [250] \\
\hline 5-Bromotetrandrine & $\downarrow$ P-gp expression & [119] \\
\hline Abietane diterpene & $\downarrow$ P-gp expression & [119] \\
\hline Amooranin & $\downarrow$ P-gp expression & [119] \\
\hline Baicalein and derivatives & $\begin{array}{c}\downarrow \text { P-gp expression } \\
\downarrow \text { MRPs }\end{array}$ & [118-120,124,141,247] \\
\hline Bitter melon extract & $\downarrow$ P-gp expression & [119] \\
\hline Bufalin & $\downarrow$ P-gp expression & [119] \\
\hline Cannabinoids & $\begin{array}{c}\downarrow \text { P-gp expression } \\
\downarrow \text { BCRP } \\
\downarrow \text { MRPs }\end{array}$ & [119] \\
\hline$\beta$-Carotene & $\downarrow$ P-gp expression & {$[101,119]$} \\
\hline Fucoxanthin & $\downarrow$ GST & [166] \\
\hline Catechins & $\downarrow$ P-gp expression & {$[111,133,143]$} \\
\hline Cepharanthine & $\begin{array}{c}\downarrow \text { P-gp expression } \\
\downarrow \text { MRP1 }\end{array}$ & [119] \\
\hline Coumarins & $\downarrow$ P-gp expression & [119] \\
\hline Cycloartanes & $\downarrow$ P-gp expression & [119] \\
\hline Didehydrostemofolines & $\downarrow$ P-gp expression & [119] \\
\hline Eudesmin & $\downarrow$ P-gp expression & [119] \\
\hline Euphocharacins A-L & $\downarrow$ P-gp expression & [119] \\
\hline Ginkgo biloba extract & $\begin{array}{c}\downarrow \text { P-gp expression } \\
\downarrow \text { MRP1 }\end{array}$ & [119] \\
\hline Grapefruit juice extracts & $\downarrow$ P-gp expression & [119] \\
\hline Hapalosin & $\downarrow$ P-gp expression & [119] \\
\hline Hypericin and hyperforin & $\begin{array}{c}\downarrow \text { P-gp expression } \\
\downarrow \text { BCRP }\end{array}$ & {$[119,246]$} \\
\hline $\begin{array}{l}\text { Isoquinoline alkaloid, } \\
\text { isotetrandrine }\end{array}$ & $\downarrow$ P-gp expression & [119] \\
\hline Isostemofoline & $\downarrow$ P-gp expression & [119] \\
\hline Jatrophanes & $\downarrow$ P-gp expression & [119] \\
\hline Kaempferia parviflora extracts & $\begin{array}{c}\downarrow \text { P-gp expression } \\
\downarrow \text { MRP1 }\end{array}$ & [119] \\
\hline
\end{tabular}


Table 1. Cont.

\begin{tabular}{|c|c|c|}
\hline Substance & Mechanism of Inhibition & References \\
\hline Kavalactones & $\downarrow$ P-gp expression & [119] \\
\hline Ningalin B and derivatives & $\downarrow$ P-gp expression & [119] \\
\hline Opiates & $\downarrow$ P-gp expression & [119] \\
\hline Piperine & $\begin{array}{c}\downarrow \text { P-gp expression } \\
\downarrow \text { BCRP } \\
\downarrow \text { MRPs } \\
\downarrow \text { ABC transporter genes } \\
\text { (ABCB1, ABCG2, and ABCC1) }\end{array}$ & [119-122] \\
\hline Polyoxypregnanes & $\downarrow$ P-gp expression & [119] \\
\hline Sesquiterpenes & $\downarrow$ P-gp expression & {$[119,247]$} \\
\hline Tenulin & $\downarrow$ P-gp expression & [104] \\
\hline Sinensetin & $\downarrow$ P-gp expression & {$[119,247]$} \\
\hline Taxane derivatives & $\downarrow$ P-gp expression & [119] \\
\hline Terpenoids & $\begin{array}{c}\downarrow \text { P-gp expression } \\
\downarrow \text { BCRP }\end{array}$ & $\begin{array}{l}{[119]} \\
{[246]}\end{array}$ \\
\hline Tetrandine & $\downarrow$ P-gp expression & [119] \\
\hline Vitamin E TPGS & $\downarrow$ P-gp expression & [119] \\
\hline $3^{\prime}-4^{\prime}-7$-Trimethoxyflavone & $\downarrow \mathrm{BCRP}$ & {$[119,141]$} \\
\hline 6-Prenylchrysin & $\downarrow \mathrm{BCRP}$ & {$[119,141]$} \\
\hline Eupatin & $\downarrow \mathrm{BCRP}$ & [119] \\
\hline Daizein & $\downarrow \mathrm{BCRP}$ & [119] \\
\hline Hesperetin & $\downarrow \mathrm{BCRP}$ & {$[119,141,244]$} \\
\hline Plumbagin & $\downarrow \mathrm{BCRP}$ & [119] \\
\hline Resveratrol & $\begin{array}{c}\downarrow \text { BCRP } \\
\downarrow \text { P-gp expression } \\
\downarrow \text { HIF- } 1 \alpha \\
\downarrow \text { GST mRNA expression }\end{array}$ & {$[114,133,140,253]$} \\
\hline Rotenoids & $\downarrow \mathrm{BCRP}$ & [119] \\
\hline Stilbenoids & $\downarrow \mathrm{BCRP}$ & [119] \\
\hline Tectochrysin & $\downarrow \mathrm{BCRP}$ & {$[119,141]$} \\
\hline Tetrahydrocurcumin & $\downarrow \mathrm{BCRP}$ & [119] \\
\hline Ligustrazine & $\downarrow$ Expression of P-gp & [7] \\
\hline Sophocarpidine & $\downarrow$ Expression of P-gp & [7] \\
\hline Strychnine & $\begin{array}{l}\downarrow \text { Gene and protein expression } \\
\text { of MRP }\end{array}$ & [7] \\
\hline Three hydroxyl soy isoflavone & $\downarrow$ MRP, MDR1, MRP2 & [7] \\
\hline Ecteinascidin & $\downarrow$ P-gp expression & [7] \\
\hline Ecteinascidin 743 & $\begin{array}{c}\downarrow \text { P-gp expression } \\
\uparrow \text { Cellular accumulation of } \\
\text { DOX/VCR in } \\
\text { P-gp-overexpressed cervix } \\
\text { cells }\end{array}$ & [118] \\
\hline $7,3^{\prime}, 4^{\prime}$-trihydroxyisoflavone & $\begin{array}{c}\downarrow \text { mRNA expression of MRP, } \\
\text { MDR1, and MRP2 }\end{array}$ & {$[7]$} \\
\hline
\end{tabular}


Table 1. Cont.

\begin{tabular}{|c|c|c|}
\hline Substance & Mechanism of Inhibition & References \\
\hline $\begin{array}{l}\text { Paeonol } \\
\text { (extracted from the dry } \\
\text { velamen of peony or any part } \\
\text { of Cynanchum paniculatum) }\end{array}$ & $\begin{aligned} \downarrow & \text { P-g expression } \\
& \downarrow \text { MDR1 } \\
& \downarrow \text { MRP } \\
& \downarrow \text { LRP }\end{aligned}$ & [7] \\
\hline Oroxylin A-7-glucuronide & $\begin{array}{c}\downarrow \text { MDR1 } \\
\downarrow \text { P-g expression }\end{array}$ & [7] \\
\hline $\begin{array}{l}3^{\prime}, 4^{\prime}, 5^{\prime}, 5,7- \\
\text { pentamethoxyflavone (PMF) } \\
\text { and derivatives of epimedium }\end{array}$ & $\begin{array}{c}\downarrow \text { MDR1 } \\
\downarrow \text { P-g expression }\end{array}$ & [7] \\
\hline $\begin{array}{c}\text { Osthole } \\
\text { (isolated from Fructus Cnidii) }\end{array}$ & $\downarrow$ P-g expression & [7] \\
\hline $\begin{array}{c}\text { Praeruptorin A } \\
\text { (extracted from Radix } \\
\text { Peucedani) }\end{array}$ & $\downarrow M D R 1$ and P-gp mRNA & {$[7,247]$} \\
\hline Diphyllin & $\downarrow$ P-gp expression & [7] \\
\hline Emodin & $\begin{array}{c}\downarrow \text { P-gp expression } \\
\downarrow \text { MRP1 } \\
\downarrow \text { GST } \pi \\
\downarrow \text { Topo II } \beta \\
\downarrow \text { HIF- } 1 \alpha\end{array}$ & {$[113,144,215,216,254-256]$} \\
\hline Psoralen & $\downarrow$ P-g expression & {$[7,242]$} \\
\hline Gypenoside & $\begin{array}{c}\downarrow \text { BCRP } \\
\downarrow \text { P-gp expression } \\
\downarrow \text { MRP1 }\end{array}$ & [7] \\
\hline Allicin & $\begin{array}{c}\downarrow \text { MDR1 } \\
\downarrow \text { P-g expression }\end{array}$ & [7] \\
\hline $\begin{array}{l}\text { Taccalonolide A and B } \\
\text { (extracted from Tacca } \\
\text { chantrieri) }\end{array}$ & $\downarrow$ P-g expression & [7] \\
\hline Oridonin & $\begin{array}{l}\downarrow \text { P-gp expression } \\
\quad \downarrow \text { GST } \pi \\
\quad \downarrow \text { LRP } 1\end{array}$ & {$[113,144,222]$} \\
\hline $\begin{array}{c}\text { Ursolic acid } \\
\text { (found in Rosmarinus } \\
\text { officinalis) }\end{array}$ & $\downarrow$ P-gp expression & {$[7,100]$} \\
\hline $\begin{array}{c}\text { Sipholenol A } \\
\text { (found in sponge Callyspongia } \\
\text { siphonella) }\end{array}$ & $\downarrow$ P-g expression & {$[113,132,146]$} \\
\hline $\begin{array}{c}\text { Cantharidin } \\
\text { (extracted from Mylabris } \\
\text { phalerata Pallas or Mylabris } \\
\text { cichorii L.) }\end{array}$ & $\downarrow$ P-g expression & [7] \\
\hline $\begin{array}{c}\text { Beta-Elemene } \\
\text { (isolated from Aeruginous } \\
\text { Turmeric rhizome) }\end{array}$ & $\begin{array}{c}\downarrow \text { P-g expression } \\
\downarrow \text { MRP }\end{array}$ & $\begin{array}{c}{[7]} \\
{[242]}\end{array}$ \\
\hline $\begin{array}{c}\text { As2O3, or white arsenic } \\
\text { Arsenic Trioxide }\end{array}$ & $\begin{array}{c}\downarrow \text { P-gp expression } \\
\downarrow \text { MRP }\end{array}$ & {$[7,242]$} \\
\hline Artemisinin & $\downarrow$ P-gp expression & [242] \\
\hline Artesunate & $\downarrow$ P-gp expression & [242] \\
\hline Baicalin & $\begin{array}{c}\downarrow \text { P-gp expression } \\
\downarrow \text { MRP1 }\end{array}$ & [242] \\
\hline
\end{tabular}


Table 1. Cont.

\begin{tabular}{|c|c|c|}
\hline Substance & Mechanism of Inhibition & References \\
\hline $\begin{array}{c}\text { Berberine } \\
\text { (isolated from ancient Chinese } \\
\text { herb Coptis chinensis French) }\end{array}$ & $\begin{array}{c}\downarrow \text { P-gp expression } \\
\downarrow \text { ABCG2 }\end{array}$ & $\begin{array}{l}{[242]} \\
{[246]}\end{array}$ \\
\hline $\begin{array}{l}\text { Carnosic acid } \\
\text { (Rosemary) }\end{array}$ & $\downarrow \mathrm{P}$-gp expression & {$[114,134,144]$} \\
\hline Chelerythrine & $\downarrow$ P-gp expression & [242] \\
\hline Gambogic acid & $\downarrow$ P-gp expression & [242] \\
\hline Neferine & $\downarrow$ P-gp expression & [242] \\
\hline Oxymatrine & $\downarrow$ P-gp expression & [242] \\
\hline Peimine & $\downarrow$ LRP & [242] \\
\hline Sodium norcantharidate & $\begin{array}{l}\downarrow \text { P-gp expression } \\
\downarrow \downarrow \text { MRP }\end{array}$ & [242] \\
\hline Brucea Javanica & $\begin{array}{c}\downarrow \text { P-gp expression } \\
\downarrow \downarrow \mathrm{MRP}\end{array}$ & [242] \\
\hline Cinobufacini & $\begin{array}{c}\downarrow \text { P-gp expression } \\
\downarrow \downarrow \text { MRP1 }\end{array}$ & [242] \\
\hline Grape seed polyphenols & $\downarrow$ P-gp expression & {$[124,242]$} \\
\hline Hyaluronate Oligomers & $\begin{array}{c}\downarrow \text { P-gp expression } \\
\downarrow \text { MRP }\end{array}$ & [242] \\
\hline Jew ear & $\begin{array}{l}\downarrow \text { P-gp expression } \\
\quad \downarrow \text { MRP }\end{array}$ & [242] \\
\hline Radix notoginseng & $\downarrow$ P-gp expression & [242] \\
\hline Rhizoma pinelliae & $\downarrow$ P-gp expression & [242] \\
\hline Realgar & $\downarrow$ P-gp expression & [242] \\
\hline Thallus laminariae & $\downarrow$ P-gp expression & [242] \\
\hline Algerian propolis & $\begin{array}{l}\downarrow \text { transport function of } \\
\text { P-gp-pump }\end{array}$ & [257] \\
\hline $\begin{array}{c}\text { Dihydroptychantol A } \\
\text { (isolated from A. angusta) }\end{array}$ & $\downarrow$ P-g expression & $\begin{array}{l}{[250]} \\
{[258]}\end{array}$ \\
\hline $\begin{array}{c}\text { Riccardin F } \\
\text { (isolated from } P . \text { intermedium) }\end{array}$ & $\downarrow$ P-gp expression & [258] \\
\hline Riccardin D & $\begin{array}{c}\downarrow \text { Topo II } \\
\downarrow \text { P-gp expression }\end{array}$ & [214] \\
\hline Andrographolid & $\downarrow$ P-gp expression & {$[94]$} \\
\hline Parthenolide & $\downarrow$ Pgp expression & {$[94]$} \\
\hline
\end{tabular}

Rhei Rhizoma, Scutellariae

Radix, Poria, Zizyphi Fructus,

Zingiberis Rhizoma, Asiasari

$\downarrow$ P-gp expression

Radix, Sophorae Radix

(herbal extract)

\begin{tabular}{ccc}
\hline Tripterygium wilfordii & $\begin{array}{c}\downarrow \text { P-gp expression } \\
\downarrow \text { EGFR }\end{array}$ & [94] \\
\hline $\begin{array}{c}\text { Shenghe Powder } \\
\text { (consisting of Radix }\end{array}$ & \\
$\begin{array}{c}\text { codonopsis pilosulae, Radix } \\
\text { pseudostellariae, Radix } \\
\text { scrophulariae, Rhizoma }\end{array}$ & $\downarrow$ P-gp expression & [94] \\
$\begin{array}{c}\text { atractylodis macrocephalae, } \\
\text { and 6 additional herbs) }\end{array}$ & & \\
\hline
\end{tabular}


Table 1. Cont.

\begin{tabular}{|c|c|c|}
\hline Substance & Mechanism of Inhibition & References \\
\hline Shen-qi-jian-wei Tang & $\begin{array}{l}\downarrow \text { MDR1 } \\
\downarrow \text { LRP }\end{array}$ & [94] \\
\hline $\begin{array}{c}\text { Yu Ping Feng San (YPFS) } \\
\text { (Astragali Radix, Atractylodis } \\
\text { Macrocephalea Rhizoma, and } \\
\text { Saposhnikoviae Radix) }\end{array}$ & $\begin{array}{c}\downarrow \text { ATP-binding cassette } \\
\text { transporters } \\
\downarrow \text { GST }\end{array}$ & [178] \\
\hline $\begin{array}{c}\text { Chinese supplement energy } \\
\text { and nourish lung (SENL) } \\
\text { herbs } \\
\text { (ginsenoside Rg1, ginsenoside } \\
\text { Rb1, ginsenoside Rg3, } \\
\text { astragaloside IV, } \\
\text { ophiopogonin D, and } \\
\text { tetrandrine) }\end{array}$ & $\downarrow \mathrm{GST} \pi$ & [193] \\
\hline Icaritin & $\downarrow$ P-gp expression & {$[118,120]$} \\
\hline Icariin & $\downarrow$ P-gp expression & [118] \\
\hline Sesquiterpene ester 1 & $\downarrow$ P-gp expression & [118] \\
\hline Celafolin A-1 & $\downarrow \mathrm{P}$-gp expression & [118] \\
\hline Celorbicol ester & $\downarrow$ P-gp expression & [118] \\
\hline Demethoxycurcumin & $\downarrow$ P-gp expression & [118] \\
\hline Euphomelliferine & $\downarrow$ P-gp expression & [118] \\
\hline Euphodendroidin D & $\downarrow$ P-gp expression & {$[118,247]$} \\
\hline Pepluanin A & $\downarrow$ P-gp expression & {$[118,247]$} \\
\hline Sipholenone E & $\downarrow$ P-gp expression & {$[118,247]$} \\
\hline Siphonellinol D & $\downarrow$ P-gp expression & [118] \\
\hline $\begin{array}{c}\text { GUT-70 } \\
\text { (From C. Brasiliense) }\end{array}$ & $\downarrow$ P-gp expression & [118] \\
\hline Lamellarin I & $\downarrow$ P-gp expression & [118] \\
\hline Wogonin & $\begin{array}{c}\downarrow \text { P-gp expression } \\
\downarrow \text { MRP1 }\end{array}$ & [118] \\
\hline Aposterol A & $\begin{array}{c}\downarrow \text { P-gp expression } \\
\downarrow \text { MRP1 }\end{array}$ & [118] \\
\hline Fumitremorgin $\mathrm{C}$ & $\begin{array}{c}\downarrow \text { BCRP } \\
\downarrow \text { P-gp expression } \\
\downarrow \text { MRP1 }\end{array}$ & {$[109,132,162]$} \\
\hline Tryprostatin A & $\downarrow \mathrm{BCRP}$ & [118] \\
\hline Terrein & $\downarrow \mathrm{BCRP}$ & [118] \\
\hline Lamellarin $\mathrm{O}$ & $\begin{array}{c}\downarrow \text { BCRP } \\
\downarrow \text { P-gp expression }\end{array}$ & [118] \\
\hline Secalonic acid D & $\begin{array}{c}\downarrow \text { BCRP } \\
\downarrow \text { P-gp expression } \\
\downarrow \text { MRP1 }\end{array}$ & [118] \\
\hline $\begin{array}{l}\text { Quinine and its isomer } \\
\text { quinidine }\end{array}$ & $\downarrow$ P-gp expression & [120] \\
\hline $\begin{array}{l}\text { Reserpine and yohimbine } \\
\text { (isolated from Rauwolfia } \\
\text { serpentine) }\end{array}$ & $\begin{array}{c}\downarrow \text { BCRP } \\
\downarrow \text { P-gp expression }\end{array}$ & [120] \\
\hline $\begin{array}{l}\text { Bromocriptine } \\
\text { ergot alkaloid }\end{array}$ & $\downarrow$ P-gp expression & [120] \\
\hline
\end{tabular}


Table 1. Cont.

\begin{tabular}{|c|c|c|}
\hline Substance & Mechanism of Inhibition & References \\
\hline$\beta$-Sitosterol-O-glucoside & $\downarrow$ P-gp expression & [120] \\
\hline cardiotonic steroid 3 & $\downarrow$ P-gp expression & [120] \\
\hline Menthol & $\downarrow$ P-gp expression & [120] \\
\hline Aromadendrene & $\downarrow$ P-gp expression & [120] \\
\hline Citronellal & $\downarrow$ P-gp expression & [120] \\
\hline Citronellol & $\downarrow$ P-gp expression & [120] \\
\hline Carnosol & $\downarrow$ P-gp expression & {$[100,120]$} \\
\hline Limonin & $\downarrow$ P-gp expression & [120] \\
\hline Kaempferide & $\begin{array}{c}\downarrow \text { BCRP } \\
\downarrow \text { P-gp expression }\end{array}$ & {$[141,244]$} \\
\hline Diosmin & $\downarrow$ P-gp expression & [244] \\
\hline Daidzein & $\downarrow \mathrm{BCRP}$ & {$[141,244]$} \\
\hline Tanshinone microemulsion & $\downarrow$ P-gp expression & [124] \\
\hline Tea polyphenol & $\downarrow$ P-gp expression & [124] \\
\hline Stemocurtisine & $\downarrow$ P-gp expression & [120] \\
\hline Stemofoline & $\downarrow$ P-gp expression & [120] \\
\hline Oxystemokerrine & $\downarrow$ P-gp expression & [120] \\
\hline $\begin{array}{c}\text { Amurensin } \mathrm{G} \text { (from Vitis } \\
\text { amurensis) }\end{array}$ & $\downarrow$ P-gp expression & [241] \\
\hline Sakuranetin & $\downarrow$ P-gp expression & [141] \\
\hline Floretin & $\downarrow$ P-gp expression & [141] \\
\hline Fisetin & $\begin{array}{c}\downarrow \text { P-gp expression } \\
\downarrow \text { GST }\end{array}$ & {$[141,192]$} \\
\hline $\begin{array}{c}\text { Xanthohumol } \\
\text { (derived from Humulus } \\
\text { lupulus) }\end{array}$ & $\begin{array}{l}\downarrow \text { mRNA expression of P-gp, } \\
\text { MRP1, MRP2 and MRP3 }\end{array}$ & [141] \\
\hline $\begin{array}{c}\text { Silybin } \\
\text { (isolated from Silybum } \\
\text { marianum) }\end{array}$ & $\begin{array}{c}\downarrow \text { MRP1 } \\
\downarrow \text { P-gp expression }\end{array}$ & [141] \\
\hline Sophoraisoflavone A & $\downarrow$ MRP1 & [141] \\
\hline $\begin{array}{c}\text { LANGDU } \\
\text { (a traditional herbal medicine) }\end{array}$ & $\downarrow$ P-g expression & [246] \\
\hline $\begin{array}{l}\text { Tanshinone IIA (isolated from } \\
\text { Salvia miltiorrhiza) }\end{array}$ & $\begin{array}{c}\downarrow \text { MRP1 } \\
\downarrow \text { BCRP } \\
\downarrow \text { P-g expression }\end{array}$ & [246] \\
\hline Auraptene (grapefruit) & $\downarrow$ P-g expression & [100] \\
\hline Nimbolide & $\downarrow$ P-gp gene & [140] \\
\hline Marsdenia tenacissima & $\begin{array}{c}\downarrow \text { P-g expression } \\
\downarrow \text { ABCG } 2 \\
\downarrow \text { MRP1 }\end{array}$ & [97] \\
\hline Taxifolin & $\begin{array}{c}\downarrow \text { ABCB1 } \\
\downarrow \text { P-gp expression }\end{array}$ & [243] \\
\hline Heterotheca inuloides Cass. & $\begin{array}{l}\downarrow \text { MDR1 } \\
\downarrow \text { MRP1 } \\
\downarrow \text { BCRP }\end{array}$ & [98] \\
\hline Saikosaponin D & $\begin{array}{c}\downarrow \text { MDR1 gene } \\
\downarrow \text { P-gp expression }\end{array}$ & [259] \\
\hline
\end{tabular}


Table 1. Cont.

\begin{tabular}{|c|c|c|}
\hline Substance & Mechanism of Inhibition & References \\
\hline $\begin{array}{c}\text { Kanglaite } \\
\text { (isolated from Coix } \\
\text { lacryma-jobi) }\end{array}$ & $\begin{array}{l}\downarrow \text { MDR1 } \\
\downarrow \text { MRP2 } \\
\downarrow \text { BCRP }\end{array}$ & [240] \\
\hline $\begin{array}{c}\text { Astragalus membranaceus } \\
\text { polysaccharides } \\
\text { Astragaloside II, another } \\
\text { component from } A \text {. } \\
\text { membranaceus }\end{array}$ & $\begin{array}{l}\downarrow \text { P-gp expression } \\
\quad \downarrow \text { MDR1 }\end{array}$ & [254] \\
\hline Wilforine & $\downarrow$ P-gp expression & [112] \\
\hline $\begin{array}{l}\text { Boswellia serrata extracts } \\
\text { 3- } \\
\text { O-acetyl-11-keto- } \beta \text {-boswellic } \\
\text { acid (AKBA), the major active } \\
\text { ingredient of the gum resin } \\
\text { from Boswellia serrata and } \\
\text { Boswellia carteri Birdw }\end{array}$ & $\downarrow$ P-gp expression & [255] \\
\hline Pervilleine F & $\downarrow$ P-gp expression & [247] \\
\hline Ellipticine & $\downarrow$ P-gp expression & [247] \\
\hline Cnidiadin & $\downarrow$ P-gp expression & [247] \\
\hline Conferone & $\downarrow$ P-gp expression & [247] \\
\hline Rivulobirin A & $\downarrow$ P-gp expression & [247] \\
\hline $\begin{array}{l}\text { Dicamphanoyl khellactone } \\
(D C K)\end{array}$ & $\downarrow$ P-gp expression & [247] \\
\hline Cannabidiol & $\downarrow$ P-gp expression & [247] \\
\hline Taccalonolides A & $\downarrow$ P-gp expression & [247] \\
\hline Jolkinol B & $\downarrow$ P-gp expression & [247] \\
\hline Portlanquinol & $\downarrow$ P-gp expression & [247] \\
\hline Dihydro- $\beta$-agarofuran & $\downarrow \mathrm{P}$-gp expression & [247] \\
\hline $\begin{array}{l}\text { Pentadeca-(8,13)-dien-11-yn- } \\
\text { 2-one }\end{array}$ & $\downarrow$ P-gp expression & [247] \\
\hline Silibinin & $\downarrow$ P-gp expression & [247] \\
\hline Nirtetralin & $\downarrow$ P-gp expression & [247] \\
\hline Cordycepin & $\downarrow$ P-gp expression & [104] \\
\hline Nuciferine & $\downarrow$ HIF- $1 \alpha$ & [235] \\
\hline Dauriporphine & $\downarrow \mathrm{P}$-g expression & [138] \\
\hline Glaucine & $\begin{array}{c}\downarrow \text { P-g expression } \\
\quad \downarrow \text { MDR1 } \\
\quad \downarrow \text { MRP1 }\end{array}$ & [138] \\
\hline Hernandezine & $\downarrow$ P-g expression & [138] \\
\hline Antofine & $\begin{array}{l}\downarrow \text { P-g expression } \\
\downarrow \text { MDR1 mRNA }\end{array}$ & [138] \\
\hline Harmine & $\downarrow \mathrm{BCRP}$ & {$[132,133,138]$} \\
\hline Tryptanthrin & $\begin{array}{c}\downarrow \text { P-g expression } \\
\downarrow \text { MRP2 }\end{array}$ & [138] \\
\hline $\begin{array}{c}\text { Lobeline } \\
\text { (from Lobelia inflate) }\end{array}$ & $\downarrow$ P-g expression & {$[134,138]$} \\
\hline
\end{tabular}


Table 1. Cont.

\begin{tabular}{|c|c|c|}
\hline Substance & Mechanism of Inhibition & References \\
\hline Tetramethylpyrazine & $\begin{array}{c}\downarrow \text { P-g expression } \\
\downarrow \text { MDR1 mRNA } \\
\downarrow \text { MRP1, MRP2, MRP3 }\end{array}$ & {$[105,138,144,238]$} \\
\hline $\begin{array}{l}\text { Danshensu and } \\
\text { tetramethylpyrazine (from the } \\
\text { Chinese herbs) }\end{array}$ & $\downarrow$ P-g expression & [238] \\
\hline Acrimarine E & $\downarrow$ P-g expression & [138] \\
\hline Gravacridonetriol & $\downarrow$ MDR1 mRNA & [138] \\
\hline 2-Methoxycitpressine I & $\downarrow$ P-g expression & [138] \\
\hline $\begin{array}{c}\text { Capsaicin } \\
\text { (extracted from Capsicum } \\
\text { annuиm) }\end{array}$ & $\downarrow$ P-g expression & {$[114,138]$} \\
\hline Acacetin & $\begin{array}{l}\downarrow \mathrm{BCRP} \\
\downarrow \mathrm{MRP} 1\end{array}$ & {$[133,138]$} \\
\hline Amorphigenin & $\downarrow$ P-g expression & [138] \\
\hline Apigenin & $\begin{array}{c}\downarrow \text { BCRP } \\
\downarrow \text { MRP1 } \\
\downarrow \text { P-g expression } \\
\downarrow \text { HIF- } 1 \alpha\end{array}$ & {$[138,143-145,240]$} \\
\hline Ampelopsin & $\downarrow$ P-g expression & [138] \\
\hline Biochanin A & $\begin{array}{c}\downarrow \text { BCRP } \\
\downarrow \text { MRP1 } \\
\downarrow \text { P-g expression }\end{array}$ & {$[132,133,138,143]$} \\
\hline Catechin & $\begin{array}{l}\downarrow \text { ATPase activity } \\
\downarrow \text { P-g expression }\end{array}$ & [138] \\
\hline Chalcone & $\begin{array}{c}\downarrow \text { MRP1 } \\
\downarrow \text { P-g expression }\end{array}$ & $\begin{array}{l}{[138]} \\
{[143]}\end{array}$ \\
\hline Chrysin & $\begin{array}{c}\downarrow \text { BCRP } \\
\downarrow \text { P-g expression }\end{array}$ & $\begin{array}{c}{[138]} \\
{[133,143]}\end{array}$ \\
\hline Diosmetin & $\downarrow \mathrm{BCRP}$ & [138] \\
\hline $\begin{array}{l}\text { Green tea catechins (EGCG, } \\
\text { ECG, CG) }\end{array}$ & $\begin{array}{c}\downarrow \text { P-g expression } \\
\downarrow \text { MDR1 } \\
\downarrow \text { ATPase activity }\end{array}$ & {$[114,143]$} \\
\hline Epicatechin gallate & $\downarrow$ P-g expression & {$[132,138]$} \\
\hline Epigallocatechin gallate & $\begin{array}{l}\downarrow \text { P-g expression } \\
\quad \downarrow \text { MDR } 1 \\
\quad \downarrow \text { ABCG } 2 \\
\quad \downarrow \text { HIF- } 1 \alpha\end{array}$ & {$[113,114,132,138,143,144,241]$} \\
\hline Formononetin & $\downarrow$ P-g expression & [138] \\
\hline Genistein & $\begin{array}{l}\downarrow \mathrm{BCRP} \\
\downarrow \mathrm{MRP} 1\end{array}$ & {$[132,133,138,143]$} \\
\hline Glabridin & $\downarrow$ P-g expression & {$[114,138,145]$} \\
\hline $\begin{array}{c}3,3^{\prime}, 4^{\prime}, 5,6,7,8- \\
\text { Heptamethoxyflavone }\end{array}$ & $\downarrow$ P-g expression & [138] \\
\hline Kaempferol & $\begin{array}{c}\downarrow \text { BCRP } \\
\downarrow \text { MRP1 } \\
\downarrow \text { P-g expression }\end{array}$ & {$[134,138,143,145]$} \\
\hline Luteolin & $\begin{array}{l}\downarrow \mathrm{BCRP} \\
\downarrow \mathrm{MRP} 1\end{array}$ & {$[138,143]$} \\
\hline
\end{tabular}


Table 1. Cont.

\begin{tabular}{|c|c|c|}
\hline Substance & Mechanism of Inhibition & References \\
\hline Morin & $\begin{array}{c}\downarrow \text { P-g expression } \\
\downarrow \text { MRP1 }\end{array}$ & {$[132,133,138,143]$} \\
\hline Myricetin & $\begin{array}{c}\downarrow \text { MRP1 and MRP2 activity } \\
\downarrow \text { Calcein efflux }\end{array}$ & {$[105,133,138,143,145]$} \\
\hline Naringenin & $\downarrow$ P-g expression & {$[134,138,143]$} \\
\hline Naringenin-7-glucosid & $\downarrow$ BCRP & [138] \\
\hline $\begin{array}{l}\text { Nobiletin (found in citrus } \\
\text { fruit) }\end{array}$ & $\begin{array}{l}\downarrow \text { P-g expression } \\
\downarrow \text { MRP1 }\end{array}$ & {$[114,138,143,146]$} \\
\hline Phloretin & $\begin{array}{l}\downarrow \text { P-g expression } \\
\downarrow \text { MRP1 }\end{array}$ & {$[132,133,138,143]$} \\
\hline Procyanidine & $\downarrow$ P-g expression & [138] \\
\hline Quercetin & $\begin{array}{c}\downarrow \text { MRP1-mediated drug } \\
\text { transport } \\
\downarrow \text { BCRP } \\
\downarrow \text { MRP1, 4 and 5. } \\
\downarrow \text { P-g expression } \\
\downarrow \text { PKC } \\
\downarrow \text { HIF- } 1 \alpha \\
\downarrow \text { MDR1 }\end{array}$ & $\begin{array}{c}{[103,111,113,132-134,138,143-} \\
146,181,182,260-262]\end{array}$ \\
\hline Robinetin & $\begin{array}{l}\downarrow \text { MRP1 and MRP2 activity } \\
\text { (inhibited calcein efflux) }\end{array}$ & [138] \\
\hline Rotenone & $\downarrow$ P-g expression & [138] \\
\hline Silymarin & $\begin{array}{c}\downarrow \text { P-gp ATPase activity } \\
\downarrow \text { P-gp-mediated cellular } \\
\text { efflux } \\
\downarrow \text { [3 H]azidopine photoaffinity } \\
\text { labeling of P-gp suggesting a } \\
\text { direct interaction with the } \\
\text { P-gp substrate binding } \\
\downarrow \text { MRP1-mediated drug } \\
\text { transport } \\
\downarrow \text { BCRP }\end{array}$ & {$[132,133,138,143,145]$} \\
\hline Tangeretin & $\begin{array}{c}\downarrow \text { P-g expression } \\
\downarrow \text { BCRP }\end{array}$ & {$[132,138,143,146]$} \\
\hline Curcumin & $\begin{array}{c}\downarrow \text { P-g expression } \\
\downarrow \text { BCRP } \\
\downarrow \text { MRP1 } \\
\downarrow \text { MDR1 mRNA } \\
\downarrow \text { ABCG2 and ABCC1 } \\
\downarrow \text { PKC- } \alpha \text { and }-\zeta \\
\downarrow \text { GST } \pi \\
\downarrow \text { Topo II } \alpha \\
\downarrow \text { HIF- } 1 \alpha\end{array}$ & $\begin{array}{c}{[103,105,113,114,132-} \\
134,138,144,180,212,252,262- \\
267]\end{array}$ \\
\hline $\begin{array}{c}\text { Matairesinol } \\
\text { (found in soybean (Glycine } \\
\text { max)) }\end{array}$ & $\begin{array}{l}\downarrow \text { P-g expression } \\
\downarrow \text { MRP1 }\end{array}$ & {$[114,138]$} \\
\hline Sesamin & $\downarrow$ P-g expression & {$[114,138]$} \\
\hline Gomisin A & $\downarrow$ P-g expression & [138] \\
\hline Schisandrol A & $\downarrow$ P-g expression & $\begin{array}{l}{[138]} \\
{[133]}\end{array}$ \\
\hline Chlorogenic acid & $\downarrow$ P-gp ATPase activity & [138] \\
\hline
\end{tabular}


Table 1. Cont.

\begin{tabular}{|c|c|c|}
\hline Substance & Mechanism of Inhibition & References \\
\hline Ginkgolic acid & $\begin{array}{l}\uparrow \text { DNR accumulation } \\
\downarrow \text { P-g expression }\end{array}$ & [138] \\
\hline Agnuside & $\downarrow$ P-gp ATPase activity & [138] \\
\hline Picroside-II & $\downarrow$ P-gp ATPase activity & [138] \\
\hline Santonin & $\downarrow$ P-gp ATPase activity & [138] \\
\hline beta-Amyrin & $\downarrow$ P-g expression & [138] \\
\hline $\begin{array}{l}\text { Glycyrrhetinic acid } \\
\text { (Enoxolone) } \\
\text { (Licorice) }\end{array}$ & $\begin{array}{l}\downarrow \text { P-g expression } \\
\downarrow \text { MRP1 }\end{array}$ & {$[114,138]$} \\
\hline Obacunone & $\downarrow$ P-g expression & [138] \\
\hline Oleanolic acid & $\downarrow$ P-g expression & {$[138,146]$} \\
\hline Uvaol & $\downarrow$ P-g expression & {$[138,146]$} \\
\hline Alisol B 23-acetate & $\downarrow$ P-g expression & {$[113,133,138]$} \\
\hline Ginsenoside $\operatorname{Rg}_{3}$ & $\begin{array}{c}\downarrow \text { Binding of }[3 \mathrm{H}] \text { azidopine } \\
\text { to P-gp } \\
\downarrow \text { P-g expression }\end{array}$ & {$[133,138]$} \\
\hline $\begin{array}{l}\text { Protopanaxatriol ginsenosides } \\
\text { 20S-ginsenoside } \\
\text { Ginsenoside Rb1 } \\
\text { Ginsenoside Rg3 }\end{array}$ & $\begin{aligned} \downarrow & \text { P-g expression } \\
& \downarrow \text { BCRP } \\
& \downarrow \text { MRP1 } \\
& \downarrow \text { MDR } 1 \\
& \downarrow \text { LRP }\end{aligned}$ & {$[113,133,138,144,146]$} \\
\hline $\begin{array}{c}\text { Tenacigenin B: P8, P26 and } \\
\text { P27 }\end{array}$ & $\begin{array}{c}\downarrow \text { P-g expression } \\
\downarrow \text { MRP1 } \\
\downarrow \text { ABCG2-mediated efflux }\end{array}$ & [138] \\
\hline Tenacigenin B: P2, P3 and P6 & $\begin{array}{c}\downarrow \text { P-g expression } \\
\downarrow \text { MRP1 }\end{array}$ & [138] \\
\hline $\begin{array}{c}\text { Tenacigenin B: P1, P4, P5, P9 } \\
\text { and P28 }\end{array}$ & $\downarrow$ P-g expression & [138] \\
\hline Aurochrome & $\downarrow$ P-g expression & [138] \\
\hline Diepoxycarotene & $\downarrow$ P-g expression & [138] \\
\hline Mutatochrome & $\downarrow$ P-g expression & [138] \\
\hline Clausarin & $\downarrow$ P-gp-mediated drug efflux & [138] \\
\hline Phyllodulcin & $\begin{array}{c}\uparrow \mathrm{DNR} \text { accumulation } \\
\text { (inhibition of P-gp-mediated } \\
\text { efflux of DNR) }\end{array}$ & [138] \\
\hline Acteoside (Verbascosine) & $\downarrow$ P-gp ATPase activity & [138] \\
\hline Berbamine & $\downarrow$ MDR1 gene expression & {$[138,144]$} \\
\hline Glaucine & $\begin{array}{c}\downarrow \text { P-g expression } \\
\downarrow \text { MRP1 } \\
\downarrow \text { MDR1 and MRP1 genes }\end{array}$ & [138] \\
\hline Fangchinoline & $\downarrow$ P-g expression & [138] \\
\hline $\begin{array}{l}\text { O-(4-ethoxyl-butyl)- } \\
\text { berbamine }\end{array}$ & $\downarrow$ MDR1 gene expression & [138] \\
\hline $\begin{array}{c}\text { Tetrandrine } \\
\text { (dried root of Stephania } \\
\text { tetrandra) }\end{array}$ & $\begin{array}{c}\downarrow \text { P-g expression } \\
\downarrow \text { LRP }\end{array}$ & {$[103,113,135,138,144]$} \\
\hline Matrine & $\downarrow$ P-g expression & [138] \\
\hline
\end{tabular}


Table 1. Cont.

\begin{tabular}{|c|c|c|}
\hline Substance & Mechanism of Inhibition & References \\
\hline Antofine & $\begin{array}{l}\downarrow \text { MDR1 mRNA } \\
\downarrow \text { P-g expression }\end{array}$ & [138] \\
\hline Ephedrine & $\begin{array}{l}\downarrow \text { MDR1 mRNA } \\
\downarrow \text { P-g expression }\end{array}$ & {$[138,144]$} \\
\hline Indole-3-carbinol & $\downarrow$ P-g expression & [138] \\
\hline Staurosporine & $\begin{array}{c}\downarrow \text { P-g expression } \\
\downarrow \text { MDR1 gene expression }\end{array}$ & [138] \\
\hline Vauqueline & $\begin{array}{l}\downarrow \text { MDR1 mRNA } \\
\downarrow \text { P-g expression }\end{array}$ & [138] \\
\hline Gravacridonetriol & $\downarrow$ MDR1 mRNA & [138] \\
\hline Clitocine & $\begin{array}{l}\downarrow \text { MDR1 mRNA } \\
\downarrow \text { P-g expression) }\end{array}$ & [138] \\
\hline Sulfinosine & $\begin{array}{l}\downarrow \text { MDR1 mRNA } \\
\downarrow \text { P-g expression }\end{array}$ & [138] \\
\hline Bisdemethoxycurcumin & $\begin{array}{c}\downarrow \text { P-gp expression } \\
\downarrow \text { MDR } 1\end{array}$ & {$[132,138]$} \\
\hline $\begin{array}{l}\text { Honokiol and magnolol } \\
\text { (isolated from Magnolia } \\
\text { officinali) }\end{array}$ & $\begin{array}{c}\downarrow \text { MDR1 } \\
\downarrow \text { P-gp expression }\end{array}$ & {$[113,138]$} \\
\hline $\begin{array}{c}\text { Schisandrin A } \\
\text { (Deoxyschizandrin) }\end{array}$ & $\begin{array}{c}\downarrow \text { P-gp expression } \\
\quad \downarrow \text { MDR1 } \\
\downarrow \text { PKC }\end{array}$ & {$[104,105,113,133,137,138,146]$} \\
\hline Schisandrin B (Sch B) & $\begin{array}{c}\downarrow \text { P-gp expression and P-gp } \\
\text { mediated efflux of Dox. } \\
\downarrow \text { MRP1 }\end{array}$ & [104] \\
\hline Triptolide & $\begin{array}{c}\downarrow \text { MDR1 } \\
\downarrow \text { MRP1 protein expression }\end{array}$ & [138] \\
\hline Pyranocoumarins & $\begin{array}{c}\downarrow \text { P-gp expression } \\
\downarrow \text { MDR1 mRNA expression }\end{array}$ & {$[133,138]$} \\
\hline $\begin{array}{l}\text { Ginger phytochemicals } \\
\text { (6-Gingerol,10- Gingerol) }\end{array}$ & $\begin{array}{c}\downarrow \text { P-gp expression } \\
\downarrow \text { MRP1 }\end{array}$ & {$[114,138]$} \\
\hline $\begin{array}{l}\text { Ginger phytochemicals } \\
\text { (6-gingerol, 10-gingerol, } \\
\text { 4-shogaol, 6-shogaol, } \\
\text { 10-shogaol, and } \\
\text { 6-dehydrogingerdione) }\end{array}$ & $\begin{array}{l}\downarrow \mathrm{GST} \pi \\
\downarrow \mathrm{MRP} 1\end{array}$ & [221] \\
\hline Alisma orientalis & $\downarrow$ P-gp expression & [252] \\
\hline Piper methysticum & $\downarrow$ P-gp expression & [252] \\
\hline Guggulsterone & $\begin{array}{c}\downarrow \text { P-gp expression } \\
\downarrow \text { MRPs }\end{array}$ & {$[113,114,134,252]$} \\
\hline Phenolic diterpenes & $\downarrow$ P-gp expression & [252] \\
\hline Vincristine & $\downarrow$ P-gp expression & [252] \\
\hline 5-Bromotetrandrine & $\downarrow$ P-gp expression & [133] \\
\hline Abietane diterpene & $\downarrow$ P-gp expression & [133] \\
\hline Amooranin & $\downarrow$ P-gp expression & [133] \\
\hline Baicalein and derivatives & $\begin{array}{c}\downarrow \text { P-gp expression } \\
\downarrow \text { MRPs }\end{array}$ & {$[103,132-134,143,146]$} \\
\hline Bitter melon extract & $\downarrow$ P-gp expression & [133] \\
\hline
\end{tabular}


Table 1. Cont.

\begin{tabular}{|c|c|c|}
\hline Substance & Mechanism of Inhibition & References \\
\hline Bufalin & $\downarrow$ P-gp expression & [133] \\
\hline Cannabinoids & $\begin{array}{c}\downarrow \text { P-gp expression } \\
\downarrow \text { BCRP } \\
\downarrow \text { MRPs }\end{array}$ & [133] \\
\hline$\beta$-Carotene & $\downarrow$ P-gp expression & {$[116,133]$} \\
\hline Fucoxanthin & $\downarrow$ GST & [201] \\
\hline Catechins & $\downarrow$ P-gp expression & {$[111,133,143]$} \\
\hline Cepharanthine & $\begin{array}{c}\downarrow \text { P-gp expression } \\
\downarrow \text { MRP1 }\end{array}$ & [133] \\
\hline Coumarins & $\downarrow$ P-gp expression & [133] \\
\hline Cycloartanes & $\downarrow$ P-gp expression & [133] \\
\hline Didehydrostemofolines & $\downarrow$ P-gp expression & [133] \\
\hline Eudesmin & $\downarrow$ P-gp expression & [133] \\
\hline Euphocharacins A-L & $\downarrow$ P-gp expression & [133] \\
\hline Ginkgo biloba extract & $\begin{array}{c}\downarrow \text { P-gp expression } \\
\downarrow \text { MRP1 }\end{array}$ & [133] \\
\hline Grapefruit juice extracts & $\downarrow$ P-gp expression & [133] \\
\hline Hapalosin & $\downarrow$ P-gp expression & [133] \\
\hline Hypericin and hyperforin & $\begin{array}{c}\downarrow \text { P-gp expression } \\
\downarrow \text { BCRP }\end{array}$ & {$[133,262]$} \\
\hline $\begin{array}{l}\text { Isoquinoline alkaloid, } \\
\text { isotetrandrine }\end{array}$ & $\downarrow$ P-gp expression & [133] \\
\hline Isostemofoline & $\downarrow$ P-gp expression & [133] \\
\hline Jatrophanes & $\downarrow$ P-gp expression & [133] \\
\hline Kaempferia parviflora extracts & $\begin{array}{c}\downarrow \text { P-gp expression } \\
\downarrow \text { MRP1 }\end{array}$ & [133] \\
\hline Kavalactones & $\downarrow$ P-gp expression & {$[133]$} \\
\hline Ningalin B and derivatives & $\downarrow$ P-gp expression & [133] \\
\hline Opiates & $\downarrow$ P-gp expression & [133] \\
\hline Piperine & $\begin{array}{c}\downarrow \text { P-gp expression } \\
\downarrow \text { BCRP } \\
\downarrow \text { MRPs } \\
\downarrow \text { ABC transporter genes } \\
\text { (ABCB1, ABCG2, and ABCC1) }\end{array}$ & [133-136] \\
\hline Polyoxypregnanes & $\downarrow$ P-gp expression & [133] \\
\hline Sesquiterpenes & $\downarrow$ P-gp expression & {$[133,146]$} \\
\hline Tenulin & $\downarrow$ P-gp expression & [107] \\
\hline Sinensetin & $\downarrow$ P-gp expression & {$[133,146]$} \\
\hline Taxane derivatives & $\downarrow$ P-gp expression & {$[133]$} \\
\hline Terpenoids & $\begin{array}{c}\downarrow \text { P-gp expression } \\
\downarrow \text { BCRP }\end{array}$ & $\begin{array}{l}{[133]} \\
{[262]}\end{array}$ \\
\hline Tetrandine & $\downarrow$ P-gp expression & {$[133]$} \\
\hline Vitamin E TPGS & $\downarrow$ P-gp expression & {$[133]$} \\
\hline $3^{\prime}-4^{\prime}-7-$ Trimethoxyflavone & $\downarrow \mathrm{BCRP}$ & {$[133,143]$} \\
\hline 6-Prenylchrysin & $\downarrow$ BCRP & {$[133,143]$} \\
\hline
\end{tabular}


Table 1. Cont.

\begin{tabular}{|c|c|c|}
\hline Substance & Mechanism of Inhibition & References \\
\hline Eupatin & $\downarrow \mathrm{BCRP}$ & [133] \\
\hline Daizein & $\downarrow$ BCRP & [133] \\
\hline Hesperetin & $\downarrow \mathrm{BCRP}$ & {$[133,143,145]$} \\
\hline Plumbagin & $\downarrow \mathrm{BCRP}$ & [133] \\
\hline Resveratrol & $\begin{array}{c}\downarrow \text { BCRP } \\
\downarrow \text { P-gp expression } \\
\downarrow \text { HIF- } 1 \alpha \\
\downarrow \text { GST mRNA expression }\end{array}$ & {$[114,133,140,253]$} \\
\hline Rotenoids & $\downarrow \mathrm{BCRP}$ & [133] \\
\hline Stilbenoids & $\downarrow$ BCRP & [133] \\
\hline Tectochrysin & $\downarrow$ BCRP & {$[133,143]$} \\
\hline Tetrahydrocurcumin & $\downarrow \mathrm{BCRP}$ & [133] \\
\hline Ligustrazine & $\downarrow$ Expression of P-gp & [113] \\
\hline Sophocarpidine & $\downarrow$ Expression of P-gp & [113] \\
\hline Strychnine & $\begin{array}{l}\downarrow \text { Gene and protein expression } \\
\text { of MRP }\end{array}$ & [113] \\
\hline Three hydroxyl soy isoflavone & $\downarrow \mathrm{MRP}, \mathrm{MDR} 1, \mathrm{MRP} 2$ & [113] \\
\hline Ecteinascidin & $\downarrow$ P-gp expression & [113] \\
\hline Ecteinascidin 743 & $\begin{array}{c}\downarrow \text { P-gp expression } \\
\uparrow \text { Cellular accumulation of } \\
\text { DOX/VCR in } \\
\text { P-gp-overexpressed cervix } \\
\text { cells }\end{array}$ & [132] \\
\hline $7,3^{\prime}, 4^{\prime}$-trihydroxyisoflavone & $\begin{array}{c}\downarrow \text { mRNA expression of MRP, } \\
\text { MDR1, and MRP2 }\end{array}$ & [113] \\
\hline $\begin{array}{c}\text { Paeonol } \\
\text { (extracted from the dry } \\
\text { velamen of peony or any part } \\
\text { of Cynanchum paniculatum) }\end{array}$ & $\begin{array}{c}\downarrow \text { P-g expression } \\
\quad \downarrow \text { MDR1 } \\
\quad \downarrow \text { MRP } \\
\quad \downarrow \text { LRP }\end{array}$ & [113] \\
\hline Oroxylin A-7-glucuronide & $\begin{array}{c}\downarrow \text { MDR1 } \\
\downarrow \text { P-g expression }\end{array}$ & [113] \\
\hline $\begin{array}{l}\qquad 3^{\prime}, 4^{\prime}, 5^{\prime}, 5,7- \\
\text { pentamethoxyflavone (PMF) } \\
\text { and derivatives of epimedium }\end{array}$ & $\begin{array}{c}\downarrow \text { MDR1 } \\
\downarrow \text { P-g expression }\end{array}$ & [113] \\
\hline $\begin{array}{c}\text { Osthole } \\
\text { (isolated from Fructus Cnidii) }\end{array}$ & $\downarrow$ P-g expression & [113] \\
\hline $\begin{array}{c}\text { Praeruptorin A } \\
\text { (extracted from Radix } \\
\text { Peucedani) }\end{array}$ & $\downarrow \mathrm{MDR} 1$ and P-gp mRNA & {$[113,146]$} \\
\hline Diphyllin & $\downarrow$ P-gp expression & [113] \\
\hline Emodin & $\begin{array}{c}\downarrow \text { P-gp expression } \\
\quad \downarrow \text { MRP } 1 \\
\quad \downarrow \text { GST } \pi \\
\downarrow \text { Topo II } \beta \\
\downarrow \text { HIF- } 1 \alpha\end{array}$ & {$[113,144,215,216,254-256]$} \\
\hline Psoralen & $\downarrow$ P-g expression & {$[113,144]$} \\
\hline Gypenoside & $\begin{array}{c}\downarrow \text { BCRP } \\
\downarrow \text { P-gp expression } \\
\downarrow \text { MRP1 }\end{array}$ & [113] \\
\hline
\end{tabular}


Table 1. Cont.

\begin{tabular}{|c|c|c|}
\hline Substance & Mechanism of Inhibition & References \\
\hline Allicin & $\begin{array}{c}\downarrow \text { MDR1 } \\
\downarrow \text { P-g expression }\end{array}$ & [113] \\
\hline $\begin{array}{c}\text { Taccalonolide A and B } \\
\text { (extracted from Tacca } \\
\text { chantrieri) }\end{array}$ & $\downarrow$ P-g expression & [113] \\
\hline Oridonin & $\begin{array}{l}\downarrow \text { P-gp expression } \\
\quad \downarrow \text { GST } \pi \\
\quad \downarrow \text { LRP1 }\end{array}$ & {$[113,144,222]$} \\
\hline $\begin{array}{c}\text { Ursolic acid } \\
\text { (found in Rosmarinus } \\
\text { officinalis) }\end{array}$ & $\downarrow$ P-gp expression & {$[113,114]$} \\
\hline $\begin{array}{c}\text { Sipholenol A } \\
\text { (found in sponge Callyspongia } \\
\text { siphonella) }\end{array}$ & $\downarrow$ P-g expression & {$[113,132,146]$} \\
\hline $\begin{array}{c}\text { Cantharidin } \\
\text { (extracted from Mylabris } \\
\text { phalerata Pallas or Mylabris } \\
\text { cichorii L.) }\end{array}$ & $\downarrow$ P-g expression & [113] \\
\hline $\begin{array}{c}\text { Beta-Elemene } \\
\text { (isolated from Aeruginous } \\
\text { Turmeric rhizome) }\end{array}$ & $\begin{array}{c}\downarrow \text { P-g expression } \\
\downarrow \text { MRP }\end{array}$ & $\begin{array}{l}{[113]} \\
{[144]}\end{array}$ \\
\hline $\begin{array}{l}\text { As2O3, or white arsenic } \\
\text { Arsenic Trioxide }\end{array}$ & $\begin{array}{c}\downarrow \text { P-gp expression } \\
\downarrow \text { MRP }\end{array}$ & {$[113,144]$} \\
\hline Artemisinin & $\downarrow$ P-gp expression & [144] \\
\hline Artesunate & $\downarrow$ P-gp expression & [144] \\
\hline Baicalin & $\begin{array}{c}\downarrow \text { P-gp expression } \\
\downarrow \text { MRP1 }\end{array}$ & [144] \\
\hline $\begin{array}{c}\text { Berberine } \\
\text { (isolated from ancient Chinese } \\
\text { herb Coptis chinensis French) }\end{array}$ & $\begin{array}{c}\downarrow \text { P-gp expression } \\
\downarrow \text { ABCG } 2\end{array}$ & $\begin{array}{l}{[144]} \\
{[262]}\end{array}$ \\
\hline $\begin{array}{l}\text { Carnosic acid } \\
\text { (Rosemary) }\end{array}$ & $\downarrow$ P-gp expression & {$[114,134,144]$} \\
\hline Chelerythrine & $\downarrow$ P-gp expression & [144] \\
\hline Gambogic acid & $\downarrow$ P-gp expression & [144] \\
\hline Neferine & $\downarrow$ P-gp expression & [144] \\
\hline Oxymatrine & $\downarrow$ P-gp expression & [144] \\
\hline Peimine & $\downarrow$ LRP & [144] \\
\hline Sodium norcantharidate & $\begin{array}{c}\downarrow \text { P-gp expression } \\
\downarrow \text { MRP }\end{array}$ & [144] \\
\hline Brucea Javanica & $\begin{array}{c}\downarrow \text { P-gp expression } \\
\downarrow \text { MRP }\end{array}$ & [144] \\
\hline Cinobufacini & $\begin{array}{c}\downarrow \text { P-gp expression } \\
\downarrow \text { MRP1 }\end{array}$ & [144] \\
\hline Grape seed polyphenols & $\downarrow$ P-gp expression & {$[103,144]$} \\
\hline Hyaluronate Oligomers & $\begin{array}{c}\downarrow \text { P-gp expression } \\
\downarrow \text { MRP }\end{array}$ & [144] \\
\hline Jew ear & $\begin{array}{c}\downarrow \text { P-gp expression } \\
\downarrow \text { MRP }\end{array}$ & [144] \\
\hline Radix notoginseng & $\downarrow$ P-gp expression & [144] \\
\hline
\end{tabular}


Table 1. Cont.

\begin{tabular}{|c|c|c|}
\hline Substance & Mechanism of Inhibition & References \\
\hline Rhizoma pinelliae & $\downarrow$ P-gp expression & [144] \\
\hline Realgar & $\downarrow$ P-gp expression & [144] \\
\hline Thallus laminariae & $\downarrow$ P-gp expression & [144] \\
\hline Algerian propolis & $\begin{array}{l}\downarrow \text { transport function of } \\
\text { P-gp-pump }\end{array}$ & [268] \\
\hline $\begin{array}{c}\text { Dihydroptychantol A } \\
\text { (isolated from A. angusta) }\end{array}$ & $\downarrow$ P-g expression & $\begin{array}{l}{[252]} \\
{[269]}\end{array}$ \\
\hline $\begin{array}{c}\text { Riccardin F } \\
\text { (isolated from } P . \text { intermedium) }\end{array}$ & $\downarrow$ P-gp expression & [269] \\
\hline Riccardin D & $\begin{array}{c}\downarrow \text { Topo II } \\
\downarrow \text { P-gp expression }\end{array}$ & [237] \\
\hline Andrographolid & $\downarrow$ P-gp expression & [105] \\
\hline Parthenolide & $\downarrow$ Pgp expression & [105] \\
\hline $\begin{array}{c}\text { Rhei Rhizoma, Scutellariae } \\
\text { Radix, Poria, Zizyphi Fructus, } \\
\text { Zingiberis Rhizoma, Asiasari } \\
\text { Radix, Sophorae Radix } \\
\text { (herbal extract) }\end{array}$ & $\downarrow$ P-gp expression & [105] \\
\hline Tripterygium wilfordii & $\begin{array}{c}\downarrow \text { P-gp expression } \\
\downarrow \text { EGFR }\end{array}$ & [105] \\
\hline $\begin{array}{c}\text { Shenghe Powder } \\
\text { (consisting of Radix } \\
\text { codonopsis pilosulae, Radix } \\
\text { pseudostellariae, Radix } \\
\text { scrophulariae, Rhizoma } \\
\text { atractylodis macrocephalae, } \\
\text { and } 6 \text { additional herbs) }\end{array}$ & $\downarrow$ P-gp expression & [105] \\
\hline Shen-qi-jian-wei Tang & $\begin{array}{l}\downarrow \text { MDR1 } \\
\downarrow \text { LRP }\end{array}$ & [105] \\
\hline $\begin{array}{c}\text { Yu Ping Feng San (YPFS) } \\
\text { (Astragali Radix, Atractylodis } \\
\text { Macrocephalea Rhizoma, and } \\
\text { Saposhnikoviae Radix) }\end{array}$ & $\begin{array}{c}\downarrow \text { ATP-binding cassette } \\
\text { transporters } \\
\downarrow \text { GST }\end{array}$ & [219] \\
\hline $\begin{array}{c}\text { Chinese supplement energy } \\
\text { and nourish lung (SENL) } \\
\text { herbs } \\
\text { (ginsenoside Rg1, ginsenoside } \\
\text { Rb1, ginsenoside Rg3, } \\
\text { astragaloside IV, } \\
\text { ophiopogonin D, and } \\
\text { tetrandrine) }\end{array}$ & $\downarrow \mathrm{GST} \pi$ & [220] \\
\hline Icaritin & $\downarrow$ P-gp expression & {$[132,134]$} \\
\hline Icariin & $\downarrow$ P-gp expression & [132] \\
\hline Sesquiterpene ester 1 & $\downarrow$ P-gp expression & [132] \\
\hline Celafolin A-1 & $\downarrow$ P-gp expression & [132] \\
\hline Celorbicol ester & $\downarrow$ P-gp expression & [132] \\
\hline Demethoxycurcumin & $\downarrow$ P-gp expression & [132] \\
\hline Euphomelliferine & $\downarrow$ P-gp expression & {$[132]$} \\
\hline Euphodendroidin D & $\downarrow$ P-gp expression & {$[132,146]$} \\
\hline Pepluanin A & $\downarrow$ P-gp expression & {$[132,146]$} \\
\hline
\end{tabular}


Table 1. Cont.

\begin{tabular}{|c|c|c|}
\hline Substance & Mechanism of Inhibition & References \\
\hline Sipholenone E & $\downarrow \mathrm{P}$-gp expression & {$[132,146]$} \\
\hline Siphonellinol D & $\downarrow$ P-gp expression & [132] \\
\hline $\begin{array}{c}\text { GUT-70 } \\
\text { (From C. Brasiliense) }\end{array}$ & $\downarrow$ P-gp expression & [132] \\
\hline Lamellarin I & $\downarrow$ P-gp expression & [132] \\
\hline Wogonin & $\begin{array}{c}\downarrow \text { P-gp expression } \\
\downarrow \text { MRP1 }\end{array}$ & [132] \\
\hline Aposterol A & $\begin{array}{c}\downarrow \text { P-gp expression } \\
\downarrow \text { MRP1 }\end{array}$ & [132] \\
\hline Fumitremorgin $\mathrm{C}$ & $\begin{array}{c}\downarrow \text { BCRP } \\
\downarrow \text { P-gp expression } \\
\downarrow \text { MRP1 }\end{array}$ & {$[109,132,162]$} \\
\hline Tryprostatin A & $\downarrow \mathrm{BCRP}$ & [132] \\
\hline Terrein & $\downarrow \mathrm{BCRP}$ & [132] \\
\hline Lamellarin $\mathrm{O}$ & $\begin{array}{c}\downarrow \text { BCRP } \\
\downarrow \text { P-gp expression }\end{array}$ & [132] \\
\hline Secalonic acid D & $\begin{array}{c}\downarrow \text { BCRP } \\
\downarrow \text { P-gp expression } \\
\downarrow \text { MRP1 }\end{array}$ & [132] \\
\hline $\begin{array}{l}\text { Quinine and its isomer } \\
\text { quinidine }\end{array}$ & $\downarrow$ P-gp expression & [134] \\
\hline $\begin{array}{l}\text { Reserpine and yohimbine } \\
\text { (isolated from Rauwolfia } \\
\text { serpentine) }\end{array}$ & $\begin{array}{c}\downarrow \text { BCRP } \\
\downarrow \text { P-gp expression }\end{array}$ & [134] \\
\hline $\begin{array}{l}\text { Bromocriptine } \\
\text { ergot alkaloid }\end{array}$ & $\downarrow$ P-gp expression & [134] \\
\hline$\beta$-Sitosterol-O-glucoside & $\downarrow$ P-gp expression & [134] \\
\hline cardiotonic steroid 3 & $\downarrow$ P-gp expression & [134] \\
\hline Menthol & $\downarrow$ P-gp expression & [134] \\
\hline Aromadendrene & $\downarrow$ P-gp expression & [134] \\
\hline Citronellal & $\downarrow$ P-gp expression & [134] \\
\hline Citronellol & $\downarrow$ P-gp expression & [134] \\
\hline Carnosol & $\downarrow$ P-gp expression & {$[114,134]$} \\
\hline Limonin & $\downarrow$ P-gp expression & [134] \\
\hline Kaempferide & $\begin{array}{c}\downarrow \text { BCRP } \\
\downarrow \text { P-gp expression }\end{array}$ & {$[143,145]$} \\
\hline Diosmin & $\downarrow$ P-gp expression & [145] \\
\hline Daidzein & $\downarrow \mathrm{BCRP}$ & {$[143,145]$} \\
\hline Tanshinone microemulsion & $\downarrow$ P-gp expression & [103] \\
\hline Tea polyphenol & $\downarrow$ P-gp expression & [103] \\
\hline Stemocurtisine & $\downarrow$ P-gp expression & {$[134]$} \\
\hline Stemofoline & $\downarrow$ P-gp expression & [134] \\
\hline Oxystemokerrine & $\downarrow$ P-gp expression & [134] \\
\hline $\begin{array}{c}\text { Amurensin } \mathrm{G} \text { (from Vitis } \\
\text { amurensis) }\end{array}$ & $\downarrow$ P-gp expression & [270] \\
\hline Sakuranetin & $\downarrow$ P-gp expression & [143] \\
\hline
\end{tabular}


Table 1. Cont.

\begin{tabular}{|c|c|c|}
\hline Substance & Mechanism of Inhibition & References \\
\hline Floretin & $\downarrow$ P-gp expression & [143] \\
\hline Fisetin & $\begin{array}{c}\downarrow \text { P-gp expression } \\
\downarrow \text { GST }\end{array}$ & {$[143,218]$} \\
\hline $\begin{array}{c}\text { Xanthohumol } \\
\text { (derived from Humulus } \\
\text { lupulus) }\end{array}$ & $\begin{array}{c}\downarrow \text { mRNA expression of P-gp, } \\
\text { MRP1, MRP2 and MRP3 }\end{array}$ & [143] \\
\hline $\begin{array}{c}\text { Silybin } \\
\text { (isolated from Silybum } \\
\text { marianum) }\end{array}$ & $\begin{array}{c}\downarrow \text { MRP1 } \\
\downarrow \text { P-gp expression }\end{array}$ & [143] \\
\hline Sophoraisoflavone A & $\downarrow \mathrm{MRP} 1$ & [143] \\
\hline $\begin{array}{c}\text { LANGDU } \\
\text { (a traditional herbal medicine) }\end{array}$ & $\downarrow$ P-g expression & [262] \\
\hline $\begin{array}{l}\text { Tanshinone IIA (isolated from } \\
\text { Salvia miltiorrhiza) }\end{array}$ & $\begin{array}{c}\downarrow \text { MRP1 } \\
\downarrow \text { BCRP } \\
\downarrow \text { P-g expression }\end{array}$ & [262] \\
\hline Auraptene (grapefruit) & $\downarrow$ P-g expression & [114] \\
\hline Nimbolide & $\downarrow$ P-gp gene & [164] \\
\hline Marsdenia tenacissima & $\begin{array}{c}\downarrow \text { P-g expression } \\
\downarrow \text { ABCG } 2 \\
\downarrow \text { MRP1 }\end{array}$ & [106] \\
\hline Taxifolin & $\begin{array}{c}\downarrow \text { ABCB1 } \\
\downarrow \text { P-gp expression }\end{array}$ & [111] \\
\hline Heterotheca inuloides Cass. & $\begin{array}{l}\downarrow \text { MDR1 } \\
\downarrow \text { MRP1 } \\
\downarrow \text { BCRP }\end{array}$ & [112] \\
\hline Saikosaponin D & $\begin{array}{c}\downarrow \text { MDR1 gene } \\
\downarrow \text { P-gp expression }\end{array}$ & [271] \\
\hline $\begin{array}{c}\text { Kanglaite } \\
\text { (isolated from Coix } \\
\text { lacryma-jobi) }\end{array}$ & $\begin{array}{l}\downarrow \mathrm{MDR} 1 \\
\downarrow \mathrm{MRP} 2 \\
\downarrow \mathrm{BCRP}\end{array}$ & [272] \\
\hline $\begin{array}{l}\text { Astragalus membranaceus } \\
\text { polysaccharides } \\
\text { Astragaloside II, another } \\
\text { component from } A \text {. } \\
\text { membranaceus }\end{array}$ & $\begin{array}{c}\downarrow \text { P-gp expression } \\
\quad \downarrow \text { MDR1 }\end{array}$ & [273] \\
\hline Wilforine & $\downarrow$ P-gp expression & [110] \\
\hline $\begin{array}{l}\text { Boswellia serrata extracts } \\
3 \text { - } \\
\text { O-acetyl-11-keto- } \beta \text {-boswellic } \\
\text { acid (AKBA), the major active } \\
\text { ingredient of the gum resin } \\
\text { from Boswellia serrata and } \\
\text { Boswellia carteri Birdw }\end{array}$ & $\downarrow$ P-gp expression & [274] \\
\hline Pervilleine F & $\downarrow$ P-gp expression & [146] \\
\hline Ellipticine & $\downarrow$ P-gp expression & [146] \\
\hline Cnidiadin & $\downarrow$ P-gp expression & [146] \\
\hline Conferone & $\downarrow$ P-gp expression & [146] \\
\hline Rivulobirin A & $\downarrow$ P-gp expression & [146] \\
\hline $\begin{array}{l}\text { Dicamphanoyl khellactone } \\
\text { (DCK) }\end{array}$ & $\downarrow$ P-gp expression & [146] \\
\hline
\end{tabular}


Table 1. Cont.

\begin{tabular}{ccc}
\hline Substance & Mechanism of Inhibition & References \\
\hline Cannabidiol & $\downarrow$ P-gp expression & {$[146]$} \\
\hline Taccalonolides A & $\downarrow$ P-gp expression & {$[146]$} \\
\hline Jolkinol B & $\downarrow$ P-gp expression & {$[146]$} \\
\hline Portlanquinol & $\downarrow$ P-gp expression & {$[146]$} \\
\hline Dihydro- $\beta$-agarofuran & $\downarrow$ P-gp expression & {$[146]$} \\
\hline Pentadeca-(8,13)-dien-11-yn- & $\downarrow$ P-gp expression & {$[146]$} \\
\hline 2-one & $\downarrow$ P-gp expression & {$[146]$} \\
\hline Silibinin & $\downarrow$ P-gp expression & {$[146]$} \\
\hline Nirtetralin & $\downarrow$ P-gp expression & {$[107]$} \\
\hline Cordycepin & $\downarrow$ HIF-1 $\alpha$ & {$[275]$} \\
\hline Nuciferine & &
\end{tabular}

\section{Synthetic Compounds in Reversing Chemo-Resistance}

Natural products are the main source of developing new drugs, and highly active phytochemicals are suitable for further modifications to formulate more effective analogs and prodrugs [260]. As mentioned, curcumin is one of the herbal remedies that has been proved to have a broad spectrum of therapeutically significant properties [253]. Interestingly, four pyrimidine-substituted curcumin analogs were tested as promising P-gp inhibitors. One of these synthetic compounds exhibited high potency and low toxicity in reversing the P-glycoprotein- mediated MDR in paclitaxel-resistant human breast cancer cells [276]. As a solution for the poor water solubility of curcumin, novel pyrazolo derivatives have been designed. They remarkably increased the sensitization of multidrug-resistant cells to doxorubicin and revealed a chance of developing potent P-gp antagonists [251]. Moreover, shikonin derivatives (acetyleshikonin and acetoxisovaleryshikonin) have been reported to trigger uptake and reduce efflux of anticancer agents in malignant carcinoma cells [277]. Additionally, novel isomers of methylated epigallocatechin and catechin have shown specific inhibition for P-gp and reversed the MDR in cancer cells [278]. Parthenolide and 5-fluorouracil conjugates have been synthesized and tested on drug-resistant hepatocellular carcinoma cells. The most active compound exhibited a high ability to inhibit MDR1, ABCC1, and ABCG2; as a result, it increased drug accumulation and induced apoptosis [275].

\section{Targeting Non-Apoptotic Cell Death Using Natural Products}

\subsection{Targeting Necroptosis}

The dynamic balance among cell proliferation, differentiation and death is of great significance in maintaining tissue homeostasis [263,264]. For a long time, apoptosis has been considered to be the single pathway in programmed cell death (PCD) $[238,261]$. In contrast, necrosis refers to the process that cells swell, rupture, and then release cellular contents and proinflammatory molecules in response to the overwhelming stress [262,265]. Necrosis is regarded as an uncontrollable process, and therefore it is highly challenging to identify small molecules that can interfere with this process [262,265]. In the late 1980s, cells under the treatment of tumor necrosis factor (TNF) were found to be characterized with either apoptotic or necrotic features in a cell type-dependent manner [266]. In 2004, Thompson et al. [252] discovered that alkylating agent $\mathrm{N}$-methyl- $\mathrm{N}^{0}$-nitro- $\mathrm{N}$-nitrosoguanidine initiated cell necrosis depending on the expression of poly ADP-ribose polymerase (PARP), suggesting that necrosis could be regulated by small molecules. After one year, the concept "necroptosis", a combination of "necrosis" and "apoptosis", was proposed [267]. Since apoptosis-resistant cases were reported in clinical cancer therapy [256], it was important and urgent to search for a new pathway to induce the death of cancer cells, while necrop- 
tosis is a promising alternative $[268,269]$. Therefore, intensive efforts and attention have been paid to investigate the mechanism of necroptosis and clarify its relationship to cancer therapy [270-272]. Recent studies indicated that necroptosis could be initiated by activation of specific receptors on cell membrane and regulated by cytokines and small molecules $[273,274,279-282]$. While the relationship between necroptosis and cancer has not been clarified explicitly, both efforts are being focused on the factors relevant to the induction and inhibition of necroptosis [283-285]. Thus, a better understanding of the mechanism of necroptosis and its modulators is important to develop novel strategies for cancer therapy.

Cell exposure to stress elicits the inherent response of cell death, which involves cell removal from the microenvironment. Necroptosis is among the several different molecular pathways and mechanisms that usually control cell death.

Recently, a necrotic process has been discovered, which is characterized by changes in the plasma membrane leading to inflammatory cell function, and it has recently been discovered that it is controlled by genes. Cytokines, pathogens, ischemia, heat, and radiation trigger various pathways (such as death receptors, kinase cascades, and mitochondria) that can induce necroptosis [286-288].

Over the last 20 years, it was found that nonapoptotic cell death can occur in a regulated fashion [289]. Chemical biology approaches were central to establishing this new paradigm, as exemplified by the study of necroptosis [290]. Necroptosis is classified as a programmed cell death in the absence of morphological traits of apoptosis or autophagy [286-288,291-294]. Emerging evidence shows that necroptosis can be disturbed in many human cancers [295-310].

Necroptosis describes regulated cell death, typically caused as a result of inflammatory pathway activation. It is a programmed means of cellular demise; the etiology is a physical insult that stimulates a signalling pathway autonomous of caspase [311]. Tumor necrosis factor a, receptor interacting protein kinase 3 , and caspase- 8 have been the subjects of considerable research in order to identify the mechanisms underlying necroptosis at a molecular level. The process may be instigated by the TNF superclass, Toll-like and interferon receptors, respectively. Necroptosis involving the former is well-delineated [287,295,312-315].

Previous studies confirmed that necroptosis was an invitation to inflammation [316]. Damage-associated molecule patterns (DAMP) are capable of recruiting primary immune cells to the necroptotic cells, followed by phagocytosis and termination of cell death signaling [317]. Furthermore, inflammation is inclined to tumorgenesis [318] and promotes cancer progression, metastasis, and increasing drug resistance [319].

Necrotic cells are eliminated from the immune system through pinocytosis or cell drinking, which is mediated by macrophages, a subcellular component of macrophages [320]. The propagation process of necroptosis is irreversible; therefore, more attention should be paid to the discovery of inhibitors to block the occurrence and execution of necrosis.

Metastasis is the primary cause of morbidity and mortality in cancer patients [321]. It was reported that shikonin dramatically reduced the metastasis of osteosarcoma C6 and U87 glioma cells due to its induction of necroptosis [310,322]. Moreover, ROS play a significant role in the migration and invasion of cells through regulation of cytoskeleton dynamics and adhesion molecules [310,323]. The generation of ROS promotes the execution of necroptosis. In other studies, RIPK3 was supposed to modulate the ROS levels in the case of necroptosis [323]. Thus, necroptosis has a close relationship with the metastasis of cancer cells.

Necroptosis serves as a back-up for apoptosis. Before the concept of necroptosis was suggested, a prevailing trend in cancer chemotherapy was to discover molecules that can induce apoptosis [324]. However, apoptosis inducers cannot work in some circumstances, such as drug resistance due to the activation of alternative compensatory pathways, upregulation of drug transporters, and multidrug resistance [324]. For example, proapoptotic agents can induce necroptosis in cancer cells when apoptosis is blocked [325]. When a 
diphtheria-based fusion toxin applied with demethylating agents, it can synergistically trigger cancer cell death and overcome apoptosis resistance by inducing necroptosis [324]. Therefore, necroptosis can serve as an alternative method in cancer therapy in place of various pathways of apoptosis.

Necroptosis may contribute to cancer progression. Necroptotic cells are characterized by cell membrane leakage and the release of molecular patterns related to intracellular damage, which result in inflammatory responses and related side effects [317]. Subsequently, they may promote tumor progression, promote tumor cell proliferation and survival, as well as tumor angiogenesis, invasion, and metastasis, and adversely affect tissues, which is not conducive to treatment [326]. Conversely, damaged and aging tissues may also promote the metastasis of cancer cells [326].

Necroptosis is not universally sensitive in tumor cell lines. Due to different environments (such as the availability of oxygen or nutrients), different cancer cell lines have different sensitivities to necroptosis [327]. Therefore, chemotherapy based on necroptosis is only effective for a limited number of tumor cell lines [327,328].

In summary, necroptosis of cancer cells could be initiated by various stimuli and through different pathways. Necroptosis is associated with tumor metastasis, which is the major cause of morbidity and mortality in cancer patients in clinic. Therefore, induction of necroptosis is an effective strategy in clinical cancer therapy. Necroptotic cells could potentially induce intrinsic and adaptive immune response and thus mediate efficient antitumor immunity. In addition, necroptosis can serve as a back-up for apoptosisresistant circumstances, which is capable of overcoming the obstacle of drug resistance that is common but intricate in clinical cancer therapy. For those apoptosis-resistant cases, necroptosis inducers could sensitize tumor cells to death, which provides another way to overcome drug resistance when common therapy fails. Thus, drug development of necroptosis inducers deserves more attention. However, the release of cytokines also induces inflammation which would be harmful to the tissue and in turn tissue damage could facilitate the metastasis of tumor cells. However, necroptosis is accompanied by the release of cytokines and induces inflammation, which is harmful to the tissue and in turn tissue damage can facilitate the metastasis of tumor cells. Additionally, it should be noted that necroptosis is not widely sensitive in cancer cells in that apoptosis is the main cause of cancer death. Therefore, necroptosis inducers are mostly favorable in apoptosis-resistant cases in clinical cancer therapy. Currently, several antitumor agents have been verified to act as necroptosis inducers, while more medicinal chemistry efforts are required to discover new inducers with drug-like properties.

Thus far, a series of necroptosis inducers have been identified, paving the way for studying new patterns of cancer death and providing new treatment tools. However, most studies are conducted in vitro, and the in vivo efficacy of necroptosis inducers and their tumor-killing selectivity still need to be further explored. In addition, there is still a lack of necroptotic markers in the body. Therefore, there is a great need to discover new necroptotic markers and study their effects on cancer cell selectivity. With a better understanding of the mechanism of cancer cell necroptosis, targeting necroptosis will become an effective strategy for cancer treatment.

As a genetically regulated mode of cellular demise, necroptosis is an essential mechanism contributing to human pathologies. A vital method of eradicating cells, the process is linked with malignancy advancement and dissemination, and immunosurveillance. Targeting the processes underlying necroptosis with small molecular modulators appears to be an encouraging strategy in oncology therapeutics; benefits include the circumvention of apoptosis resistance and ongoing anticancer immunity integrity. Thus, in order to design de novo approaches for tumor treatment, an improved comprehension of necroptosis and its governing molecules is required.

While numerous reviews focused on the apoptotic pathways, extrinsic and intrinsic alike, few articles concentrated on necroptosis induced by natural compounds in tumor 
cells [329-334]. Table 2 shows a list of natural compounds with an effect on non-apoptosis cell death in tumor cells.

Natural compounds originating from plants, microorganisms, and marine life forms were widely shown to display anti-carcinogenic, anti-proliferative, and anti-survival effects inducing tumor cell death via various pathways, including necroptosis, leading to cell death [329-343].

An alkaloid extracted from traditional Chinese herb Sophora flavescens, called matrine, was reported to induce necroptosis in cholangiocarcinoma cells, which was different from its classic apoptosis-inducing effects in other cancer cell lines [344]. Mz-ChA-1 and QBC939 cells (cholangiocarcinoma cells) treated with matrine were characterized by extensive organelles, plasma membrane rupture, and integral nuclei which coincided with the morphology of necroptotic cells [344]. Additionally, cells that died due to the treatment of matrine could not be rescued by the addition of pan-caspase inhibitor 17, but they could be rescued by necroptosis inhibitor 2 [344]. Further studies confirmed that matrine induces necroptosis through the formation of RIPK1/RIPK3/MLKL complex [344]. Osmotic pressure and release of ROS also facilitated the necroptotic process [344]. More interestingly, matrine was able to upregulate the expression of RIPK3 in Mz-ChA-1 cells, making it possible to treat cholangiocarcinoma with a low expression of RIPK3 [344]. This work potentially provided a new strategy to deal with the apoptosis resistance in cholangiocarcinoma therapy [344].

Neoalbaconol, extracted from Albatrellus confluens, was reported to trigger several kinds of cell death [345]. NA downregulates the E3 ubiquitin ligase, resulting in a decrease in RIPK1 ubiquitination. Therefore, an increase in the expression level of RIPK1 was observed, which also activated the transcription of TNF-a [345]. In addition, NA causes RIPK3-mediated ROS generation, which contributes to cell death [345]. Therefore, it can be concluded that NA can induce necroptosis by activating TNF- $\alpha$ and RIPK3-dependent ROS production [345].

Shikonin, extracted from the traditional Chinese herb "Zicao", was originally used to treat wound healing because of its anti-inflammatory and antimicrobial properties [346]. It was reported to kill tumor cells by inducing apoptosis [346]. Huang et al. [322] found that losses in plasma membrane integrity and intact nuclear membranes in glioma cells were observed in cells treated with shikonin directly by electronic transmission microscopy. Shikonin-induced C6 and u87 glioma cells can be rescued by necroptosis inhibitor 2, but are unaffected by treatment with the caspase inhibitor 17. Additionally, an increased expression of RIPK1 was observed after treatment with shikonin [322]. All these facts indicate that shikonin induces necroptosis through RIPK1 activation.

Emodin, an anthraquinone derivative extracted from traditional Chinese medicine Rheum palmatum, has been used to treat various diseases due to its antitumor, antiinflammation, anti-metastasis, and immunosuppressive effects, but its mechanism of action remains unclear [347]. Zhou et al. [348] found that cells treated with emodin showed increased levels of RIPK1, RIPK3, and TNF- a. In addition, the combined use of emodin and necroptosis inhibitor 2 or 15 reduced the release of lactate dehydrogenase (LDH) [348]. Hematoxylin-eosin (H\&E) staining of tumor tissue isolated from mice treated with emodin showed an obvious necrotic effect [348]. Further studies indicated that TNF-a, RIPK1, RIPK3, and MLKL in tumor tissues were upregulated when treated with emodin, indicating that it could inhibit glioma growth in vivo through necroptosis [348].

Ungeremine is an alkaloid extracted from Ungernia minor, which shows effective cytotoxicity in drug-resistant cancer cell lines and upregulates RIPK3 levels [349]. Cotreatment with Necroptosis Inhibitor 2 reduced the cytotoxicity of ungeremine, and flow cytometry analysis showed that $13.1 \%$ of the cells were necrotic [349].

Several natural compounds were shown to induce necroptosis in human tumor cells. For example, shikonin, a naphthoquinone derived from Lithospermum erythrorhizon Siebold \& Zucc. (Zicao), was found to be the first natural product capable to induce both apoptosis and necroptosis in human several cancer cell lines [310,322,350-361]. Shikonin- 
treated cells showed morphological alterations distinct from those occurring in apoptosis or autophagic cell death [310,322,350-361]. The loss of plasma membrane integrity was one of the morphologic characteristics of necrotic cell death [310,322,350-361]. Shikonin exerts a dramatic anticancer effect on both primary and metastatic osteosarcoma by inducing RIPK-1 and 3- dependent necroptosis [310,351]. The size of primary osteosarcoma tumors and lung metastases was significantly reduced by shikonin treatment accompanied the elevated levels for RIPK-1 and RIPK-3 proteins [310,351]. Shikonin was observed to induce necroptotic cell death in tumor cells involving drug- and apoptosis-resistant cancer cells that overexpress multidrug resistance protein-1, P-glycoprotein, and other proteins [310].

Shikonin exerts effective cytotoxicity on human multiple myeloma borate-resistant KMS11/BTZ cells [353]. Shikonin can circumvent the drug resistance displayed by tumor cells and mediated by P-glycoprotein, BCL-2, and BCL-xL by inducing necroptosis [355-359]. Naturally occurring shikonin analogues (deoxyshikonin, acetylshikonin, isobutyrylshikonin, $\beta, \beta$-dimethylacryloylshikonin, isovalerylshikonin, $\alpha$-methyl-nbutylshikonin) induce necroptosis in resistant tumor cells overexpressing MDR1 and BCRP1 [322,352-358].

Staurosporine, a protein kinases inhibitor, induces necroptosis in leukemia cells when caspase activation is inhibited [362]. It was recently determined that the TNF-related apoptosis-inducing ligand (TRAIL) induces necroptosis via RIPK-1-/RIPK-3-dependent PARP1 activation, consequently turning PARP1 activation into an effector mechanism downstream of RIPK-1 [363,364]. Obatoclax (GX15-070), a small-molecule inhibitor of antiapoptotic BCL-2 proteins, was shown to induce autophagy-dependent necroptosis in glucocorticoid-resistant acute lymphoblastic leukemia in childhood [365-367]. It was further observed to induce an assembly of necrosomes on autophagosomal membranes [365]. It increased the physical association of ATG5 with RIPK-1 and 3 [365]. Furthermore, photodynamic therapy by incorporating 5 -aminolevulinic acid was noted to trigger RIPK3-mediated necrosis in glioblastoma cells [368].

Piperlongumine (a natural constituent of the fruit of Piper longum), and taurolidine (a derivative of taurine) were observed to inhibit in vitro and in vivo cancer cell growth [369]. Piperlongumine can selectively kill specific types of cancer cells over normal cells [369]. It inhibits the growth of xenografted human malignant breast tumors in vivo [369]. Taurolidine induces cytotoxicity in different cancer cells in vivo and in vitro [369]. The effect of piperlongumine and taurolidine is dependent on the stimulation of programmed cell death by autophagy via a redox-directed mechanism, in addition to apoptosis and necroptosis [369]. A decrease in the ROS levels markedly reduces the AA005-mediated cell death in SW620 cells [370]. Additionally, the RIPK-1 repression by NEC-1 prevents the translocation of the apoptosis-inducing factor and partially destroys AA005-induced cell death in SW620 cells, suggesting a role for the necroptotic pathway [370].

Eupomatenoid-5 (Eup-5) was found to induce cell death in renal cancer 786-0 cells in addition to human breast tumor MCF-7 cells [371]. In MCF-7 cells, Eup-5 caused phosphatidylserine externalization and caspase activation, while 786-0 cells treated with Eup-5 showed characteristics of the programmed necroptosis process [371]. Green tea polyphenols were shown to induce BAX and BAK activation, cytochrome $\mathrm{c}$ release, caspase activation, and necroptosis of human hepatocellular carcinoma Hep3B cells [370]. Interestingly, in Hep3B cells lacking BAX (BAX-/-) or BAK (BAK-/-) expression, cytochrome $\mathrm{c}$ release and necroptosis were reduced [372].

Concurrent necrosis and apoptosis have been demonstrated following parthenolide in human acute promyelocytic HL-60 and Jurkat T lymphoma cells, respectively [373]. Membrane breach and swift necrotic cell demise resulted from the engagement of parthenolide with the cell membrane [374]. In human MDA-MB231 breast cancer cell lines, necrosis induced by parthenolide was diminished by NAC and NEC-1, thus inferring that ROS and RIP-1 stimulation may be relevant [375]. 


\subsection{Targeting Autophagy}

Autophagy, formerly termed macroautophagy, is a further essential mode of malignant cell demise following chemotherapy. It is characterized by self-destructing intracellular proteins and organelles within the lysosome. The process is flexible and necessary for cellular equilibrium, stimulating cellular demise without the presence of apoptotic moderators but when vital autophagy-governed genes, e.g., ATG5 [376] and BECN1 [377], are evident.

Autophagy is the process by which cells forms double-membrane autophagic vesicles $(\mathrm{AV})$, which isolate organelles and proteins and target them for degradation in the lysosome. Although it was originally viewed as a "bulk degradation" process activated by cellular starvation, new findings demonstrate that autophagy can also be a highly selective qualitycontrol mechanism that regulates levels of specific organelles and proteins [378-381].

Exhaustion of nutrients is maintained by the highly conserved adaptive process called autophagy. This process is exploited by solid tumor cells to achieve homeostasis through cytoplasmic waste metabolism to satisfy growing demand for nutrients. This promotes development of cellular stressors such as inflammation, hypoxia, and tumor progression. Different solid tumors have been found to have poor prognosis depending on the extent of autophagy [382-385].

Autophagy can be categorized as microautophagy, macroautophagy, and chaperonemediated autophagy; all classes frequently enhance the proteolytic eradication of cytosolic constituents within the lysosome [386-388]. Contemporary work has concentrated particularly on macroautophagy, including delineation of its properties at cellular and molecular levels, and its potential use in antitumor treatment [389,390].

In cancer, autophagy may play a role in limiting the earliest stages of tumorigenesis; however, there is growing evidence that, in established cancers, autophagy can help cope with intracellular and environmental stresses, such as hypoxia, nutrient shortage, or cancer therapy, thereby favoring tumor progression as a survival mechanism. In this context, it is becoming increasingly clear that autophagy inhibition could improve therapeutic outcomes for patients with advanced cancer.

In contrast to normal cells, tumor cells frequently exhibit impaired autophagic processes, although the relevance of autophagy in malignancy is contradictory to that in the normal cellular state. Current evidence implies that autophagy may both advance and hinder tumor development by enhancement of neoplastic cell longevity and a cancersuppressive action in non-malignant cells, respectively [391]. Elimination of structurally compromised proteins and organelles may inhibit oncogenesis and enable existing cancers to survive nutrient or oxygen lack during their growth [392].

Our current understanding is that the autophagy pathway consists of at least seven steps [393-401]. The conserved autophagy genes (ATG genes) regulate steps 1 to 5:

Step 1: "The Unc-51-like kinase protein kinase complex": regulates initiation of AV formation.

Step 2: "The VPS34 lipid kinase complex": prepares the membrane for curvature.

Step 3: "LC3 family conjugation cascade".

Step 4: "Cargo loading through autophagy cargo adaptors".

Step 5: "AV maturation".

The genes that are common to other endosomal/lysosomal pathways promote steps 6 and 7.

Step 6: "AV-lysosome fusion".

Step 7: "Lysosomal degradation and recycling of AV cargo".

Although these seven steps of autophagy are well documented, it is expected that additional regulators will be discovered. New, novel regulators of autophagy were recognized using an siRNA screen in a pancreatic cancer cell line. Two of the promising candidates, MAGUK p55 subfamily member 7 (MPP7) and cytosolic malate dehydrogenase 1 (MDH1), were observed to have roles in forming the autophagosome [402]. Many of these steps in the autophagy pathway represent potentially druggable targets providing 
ways to both positively and negatively influence autophagy. As autophagy is a complex multistep mechanism, understanding the details of autophagy is critical to developing effective compounds and therapies to modulate autophagy potently and specifically.

Our current understanding is that the autophagy pathway consists of at least seven steps [403-411]. The conserved autophagy genes (ATG genes) regulate steps 1 to 5: Step 1: The Unc-51-like kinase protein kinase complex regulates the initiation of AV formation. Step 2: The VPS34 lipid kinase complex prepares the membrane for curvature. Steps 1 and 2 arrange intracellular membranes to form $\mathrm{AVs}$ via enriching the membrane for phosphatidylinositol 3 phosphate (PI3P). Step 3: LC3 family conjugation cascade. Step 4: Cargo loading through autophagy cargo adaptors. Step 5: AV maturation. The genes shared by other endosomal/lysosomal pathways facilitate steps 6 and 7: Step 6: AV-lysosome fusion. Step 7: Lysosomal degradation and recycling of AV cargo. Although these seven steps of autophagy are well documented, it is expected that additional regulators will be discovered. New, novel regulators of autophagy were recognized using an siRNA screen in a pancreatic cancer cell line. Two promising candidates, members of the MAGUK p55 subfamily 7 (MPP7) and cytosolic malate dehydrogenase 1 (MDH1), were observed to have roles in forming the autophagosome. MPP7 activates YAP1, induces autophagy, and MDH1 regulates ULK1 levels [412]. Many of these steps in the autophagy pathway represent potentially druggable targets providing ways to both positively and negatively influence autophagy. As autophagy is a complex multistep mechanism, understanding the details of autophagy is critical to developing effective compounds and therapies to modulate autophagy potently and specifically.

The value of autophagy in health and disease was recently emphasized when Yoshinori Ohsumi received the Nobel Prize in Medicine in 2016 for his work on advancing understanding of the genetic foundation of autophagy in yeast [413]. Multiple groups were involved in endeavors to demonstrate that [403].

A number of human pathologies are associated with autophagic anomalies, e.g., dementia, cardiovascular conditions, leishmaniasis, influenza, liver disorders and malignancy, e.g., hepatocellular carcinoma (HCC).

The fifth leading form of fatal hepatic malignancy worldwide, HCC, has a heterogeneous geographical prevalence, affecting males three-fold more than females. Current therapeutic interventions have a range of clinical outcomes; short survival times result from pharmaceutical resistance and adverse events.

Autophagy is one of the underlying disease processes that is targeted during the design of antitumor agents. Overall, overstimulated autophagy may precipitate a nonapoptotic type of programmed cell death (PCD), autophagic or type II PCD. Recent studies have inferred that malignant cell type II PCD can be initiated through autophagic interference, thus inhibiting cancer progression. Influencing associated signalling is therefore an encouraging route for the generation of de novo agents to combat drug-resistant tumor cells.

Natural polyphenolic substances, e.g., flavonoids and non-flavonoids, exert an antitumor action through the upregulation of cancer suppressors and autophagy mediated via signalling pathways that are both canonical (Beclin 1-dependent) and non-canonical (autonomous of Beclin-1). Angiogenesis and tumor dissemination in HCC have been shown to be targets of plant polyphenols; these influence numerous intracellular mediators and diminish HCC risk [404].

Some research, particularly from Kroemer's group, has postulated that autophagy suppression would be detrimental to tumor therapy as it would diminish the responses of antitumor T cells [405-407]. In expiring malignant cells, autophagy is believed to be necessary for immunogenic cellular demise, giving rise to cellular identification by the immune system and stimulation of an efficacious immune reaction [408,409]. However, this research was conducted in extremely immunogenic experimental models [410], which may have influenced the data. In contrast, research employing murine tumor models of lower immunogenicity demonstrated comparable $\mathrm{T}$ cell responses between animals 
with and without effective autophagy; in the latter, genetic deletion of relevant genes or pharmacological autophagy inhibition with CQ was applied [411].

Progressing the concept of the necessity of autophagy for immunogenic cell eradication, Kroemer et al. surmised that autophagy, upregulated utilizing caloric restriction mimetics, could uplift anti-neoplastic immune responses [412], implying that autophagy should not be suppressed and that anticancer therapies should be potentially targeted towards its enhancement.

Thus, although here only contemplating anticancer immune reactions, inhibition of autophagy remains the subject of debate [414-416] and could have the opposite effect to that desired from first line treatment [417-419].

A further issue relating to targeting autophagy is that in patients, autophagy suppression would not be restricted to cancerous cells, thus causing toxicity from more widespread effects. For instance, research has demonstrated that in adult mice, global tissue knockout of Atg7, a critical autophagy gene, caused their ultimate demise as a consequence of grave neuronal dysfunction, loss of glucose metabolism, and enhanced vulnerability to infection [420].

Nevertheless, although extracting a vital constituent of the canonical pathway of autophagy from each cell may mimic the impact of a 'flawless' autophagy suppressor, this approach is at variance with clinical applications where a pharmaceutical agent would be less potent than total knockout of a key autophagy moderator.

Reinforcing this concept, long-term HCQ utilisation in rheumatological conditions and CQ therapy in certain malignancies as autophagy suppressors has not been associated with intolerable toxicity [421], showing that ongoing lysosomal autophagy inhibition is possible. Essentially, if malignant tissues have a higher reliance on autophagy than normal cells, an agent that gives rise to toxicity in the latter may have a clinically appropriate treatment period for efficacious tumor therapy. In fact, in the $A g 7$ knockout model, KRAS-driven lung cancers were notably suppressed by gene deletion prior to evidence of neurotoxicity [420], confirming the potential presence of a therapeutic window for autophagy suppression in certain malignancies.

The precise function of autophagy in controlling malignant immune responses remains obscure and the controversy regarding autophagy suppression continues. Additional studies are merited to characterize its associated hazards and application whilst simultaneously heightening immune responses to obtain positive clinical endpoints. Markers denoting autophagy dependence may be useful for the recognition of individuals who would gain most benefit from treatment by autophagy inhibition.

Autophagy can help cancer cells to survive and withstand the action of therapeutic agents in the context of chemotherapy-activated cellular stress. A correlation between extensive autophagy and systemic therapy resistance, including androgen inhibition therapy in prostate cancer, has been highlighted by a number of clinical and preclinical models [422-430].

A very large body of literature supports the induction of autophagy during cancer therapy as a key resistance mechanism in multiple cancer types. Many of the stresssensing pathways that induce autophagy are engaged by cancer therapies used in the clinic. Although complete and prolonged autophagy can produce cell death in vitro and in vivo, the major role of autophagy induction is to enable survival of the cancer cell during therapy. For example, cisplatin-induced autophagy was shown to play a role in tumor cell resistance to platinum-based therapy [431]. Cytotoxic chemotherapy, targeted therapy, and radiotherapy can all activate cytoprotective autophagy [432].

Emerging evidence suggests that autophagy is upregulated in tumor cells in response to various stresses, leading to tumor cells becoming resistant to chemotherapy. Therefore, targeting the autophagy signaling pathway may be an innovative strategy for human cancer prevention and combination therapy and for combating tumor-derived chemoresistance $[433,434]$. 
Increasing evidence suggests the possibility that autophagy may be a key actor in the evolution of drug resistance in tumor cells. Enhanced autophagy was demonstrated in individuals with melanoma in who neoplastic cells developed resistance to vemurafenib, a BRAF inhibitor, through an ER stress reaction [435]. Additionally, vemurafenib resistance induced by sustained melanoma cell line culture with the agent could be abolished via autophagy suppression [435]. Clinically, a patient with BRAF-mutant cerebral malignancy, in whom vemurafenib was at first successful but then hindered by drug resistance, had a positive outcome when prescribed CQ as an adjunctive therapy [421]. This case report demonstrated resensitisation of a malignancy by autophagy inhibition. Of note, was that the admixture of kinase and autophagy inhibitors, and not the latter alone, was beneficial for the ongoing suppression of cancer progression, suggesting that the clinical advantage pertains to surmounting drug resistance as opposed to engineering de novo responsiveness to sole treatment with an autophagy suppressor [421].

Additionally, the contribution of autophagy to multi-drug resistance, frequently to routinely used agents employed for challenging malignancies, has been inferred. In ovarian cancer, provocation of autophagy has underpinned resistance to paclitaxel, a cytotoxic agent [436]. Similarly, this process has been demonstrated to underlie cisplatin resistance in ovarian and oesophageal cancers, and occur as a result of low oxygen levels in lung tumors [437]. In primary chronic lymphocytic leukemia cells in patients, precipitation of autophagy owing to an ER stress reaction, as in melanoma, leads to resistance to cyclin-dependent kinase (CDK) inhibitors [438] and to resistance to HDAC inhibitors, e.g., tubastatin A, in cell lines derived from glioblastomas [439]. The connection between autophagy and chemotherapy resistance continues to grow and so autophagy, as a potential target for antitumor agents, will inevitably remain the subject of ongoing research [440-444].

Autophagy has additionally been associated with promotion of dormant cancer cell survival and may form a key enabler of cell growth recommencement [445]. Contemporary work on a malignant drosophila model demonstrated that quiescent neoplastic tissue from autophagy-deficient flies was initiated into growth when relocated into autophagycompetent organisms. These results implied that non-malignant cell independent autophagy in the environs' adjacent cells is essential for growth reestablishment in quiescent neoplasia [446]. If comparable events take place in mammals, this study would indicate that endeavors to promote autophagy following a presumed positive outcome from tumor therapy may have the undesired consequence of precipitating recurrence by stimulating remaining dormant malignant tissue.

Autophagy may be an efficacious tumor escape mechanism; it is thought to contribute to resistance evolution in numerous malignancies, e.g., those originating in the central nervous system, melanoma, non-small cell lung cancer (NSCLC), and neoplasia of the bladder and thyroid. Concomitant autophagy inhibition therapy in these instances may diminish or rectify treatment resistance.

In the oncology sector, autophagy has conflicting and setting-reliant influences and so a 'one size fits all' strategy with therapies engineered to suppress or to promote this process in tumor treatment will not have a positive outcome. In view of this realization, the optimal approach could be to circumvent interference with autophagy altogether in this context. Nevertheless, changes to autophagy are inevitable, as it is impacted by numerous contemporary therapies. Furthermore, physiological triggers, particularly those that frequently exert diverse influences on malignant and normal cells, respectively, e.g., lack of nutrients or oxygen, will additionally impact the autophagic process in neoplastic tissue. Thus, it is important to delineate the consequences of these alterations and to attempt to customize treatments to suit specific contexts. To begin with, such therapies will potentially center on autophagy suppression; a major requirement is then to determine which patients would benefit from this strategy.

It has been discovered that a variety of compounds can induce tumor cell death by deregulating the signaling pathways that lead to autophagy [329,333,334,447-453] 
(Table 2). Melatonin promoted vincristine-resistant oral cancer cell autophagy and apoptosis and reduced the drug resistance of vincristine-resistant oral cancer cells by increasing the expression of microRNA [454].

An autophagy-mediated mode of cell death is generated by the application of a number of polyphenolic substances in a range of malignant cells [455]. A category of natural organic chemicals, polyphenols typically comprise numerous phenol structural moieties and are present in the diet. Several, e.g., rottlerin [456], curcumin [457], resveratrol [458], genistein [459,460], and quercetin [461] exhibit antitumor properties, influencing signalling pathways and promoting cell death through both apoptotic and autophagic processes [462,463]. These substances can stimulate type II PCD through a spectrum of mechanisms via canonical (Beclin-1-dependent) and non-canconical (autonomous of Beclin-1) autophagic avenues (212), and have the potential to be utilized as adjuncts to routine antitumor treatments.

Polyphenols additionally moderate autophagy in order to surmount or to reverse cellular multi-drug resistance. Apigenin notably enhances the responsiveness of doxorubicinresistant BEL-7402/ADM cells, triggers miR-520b expression, and inhibits Atg7 [464]. Interference with autophagy gives rise to PARP-1 upregulation in ovarian tumor cells undergoing therapy with cisplatin; this is essential for cellular longevity. PARP-1 suppression, at mRNA and protein junctures, together with autophagy inhibition, can be achieved using luteolin, thus returning sensitivity to cisplatin [465]. An active flavone, scutellarin has been demonstrated to diminish cell cycle-associated Cdc2 and cyclin B1 protein expression, to activate apoptosis in PC3 cells, and to preserve resistance to cisplatin [465].

Naturally occurring polyphenols, flavonolignans, comprise flavonoid and lignin portions. Originating from silymarin extract, the flavonolignan, silibinin, displayed antigenotoxic, membrane-stabilizing, and anti-oxidant properties and triggered liver cell regrowth, inhibited fibrogenesis, and reduced the intrahepatic inflammatory response. The compound has been suggested to activate autophagy in HeLa cervical malignancy [466] and in MCF-7 breast tumor cell lines via the generation of LC3-II, Atg12-Atg5 and Beclin-1 upregulation [467]. Autophagy was promoted by silibinin in human fibrosarcoma HT1080 cells via ROS/p38/JNK pathway-induced p53 stimulation [468], in A375-S2 cell lines from melanoma [469], and in SW480 and SW620 cells from colonic tumors [470]. Since silibinin is a potent autophagic stimulator within a range of cell types, its potential to have an anti-HCC impact through this action merits further investigation.

One of the most significant oncological issues requiring resolution is the rise in cancer resistance to chemotherapeutic agents, radiotherapy, or targeted interventions. Polyphenolic substances are able to stimulate apoptosis and autophagy, thus promoting malignant cell death. As dietary components, their intrinsically diminished toxicity, facilitation of lower dosages, and adverse events compared with man-made agents denotes them as low risk. Admixing them with treatments sanctioned by the Food and Drug Administration (FDA) may offer de novo approaches for malignancy therapy and a counter strategy against the significant issue of drug resistance.

Numerous papers have examined the natural phytopolyphenol, curcumin, in terms of its antitumorigenesis and therapeutic properties [471]. Administration of curcumin to Huh7 cells precipitated early autophagy, as evidenced by autophagic vacuolar evolution [472]. In male Sprague-Dawley rodents, survival statistics for thioacetamide-induced HCC were improved following curcumin by autophagy signalling pathway stimulation via protein expression and apoptosis suppression [473]. Adriamycin (doxorubicin) and curcumin together induce autophagy associated with a raised prevalence of autophagosomes in treated juxtanuclear cells. Research evaluating the autophagy suppressor, 3-MA, has also demonstrated the value of combination therapy [474].

Rottlerin, or mallotoxin, is derived from the monkey-faced tree, Mallotus phillippinensis [475]. Numerous signalling pathways and cellular mechanisms contribute to autophagy and the consequent cellular demise stimulated by this compound. Nevertheless, cellular setting, heightened threshold or resistance to apoptosis and stimulated or suppressed sig- 
nalling pathways are probably the key elements dictating cellular destinies. Rottlerin and associated analogues may be utilized in the configuration of new therapies for autophagy induction in prostate [476] and pancreatic [477] tumors.

Present in soy items, genistein is a naturally arising isoflavonoid noted to exhibit antitumor activities, including the ability to promote cellular demise via apoptosis [478] and autophagy [479]. The latter arises owing to alterations in apoptotic signalling and is advantageous against malignancy cell resistance to chemical agents [480]. During stress, conditions of poor nutrients or a lack of growth factor, genistein can safeguard the cytokeratin matrix. Several researchers have demonstrated its value in surmounting the deleterious consequences of the potent autophagic inhibitor, okadaic acid, on cytoskeletal and cytokeratin configurations in rodent liver cells, which is of note as the latter contributes to autophagic evolution. Despite its autophagic and apoptotic properties in tumor cells, genistein has been shown to have inherently poor oral bioavailability owing to metabolic enzymes and efflux transporters. This issue merits additional study in order to enhance its effectiveness for the therapy of apoptotic-resistant neoplasia [481].

Quercetin is a natural flavonoid molecule found in fruits, vegetables, leaves, and grains. It has antitumor effects related to its ability to target key molecules, organelles, and tumorigenic pathways [482]. Quercetin mediates extensive autophagy and subsequent death in cancer cells by inhibiting proteasome activity [480]. Many studies have shown that quercetin has an effect on autophagy [483,484].

Resveratrol, a natural polyphenol, did not show autophagic response at low concentrations $(10 \mathrm{pg} / \mathrm{mL})$; however, at a higher concentrations $(20 \mathrm{pg} / \mathrm{mL})$, it activated autophagic cell death in Huh 7 cells [485]. Resveratrol may be effective therapy in apoptosis-resistant ovarian cancer as its acute exposure induces cell death through autophagy in five ovarian cancer cell lines. [403,482,486]. Resveratrol exhibited an effect against human hepatocellular carcinoma (HCC) by inducing autophagy [487]. Resveratrol enhanced the expression of several tubulin subunits that is important for autophagosomes movement inside the cell. Furthermore, there is evidence that resveratrol triggers autophagic death in the cells of chronic myeloid leukemia [488,489]. Trincheri et al. [490] reported that autophagy can be induced with acute exposure to resveratrol.

Autophagy induced in human glioma cells by resveratrol has the ability to inhibit resveratrol-induced apoptosis [491]. Autophagy inhibitors may have the potential to enhance resveratrol antitumor efficacy [491] because autophagy delayed apoptosis and protected the cells from death.

Persistent human papillomavirus infection may stabilize an anti-autophagy factor called ATAD3A, inhibit cell apoptosis in addition to autophagy, and increase drug resistance in uterine cervical cancer. Resveratrol's antitumor activity was confirmed by its ability to reduce ATAD3A expression and to increase the numbers of autophagosomes [492].

Resveratrol activates autophagic cell death in human prostate cancer PC3 and DU145 cells by downregulating matrix-interacting molecules 1 (STIM1) expression leads to the induction of endoplasmic reticulum stress, which activates AMPK and inhibits the AKT/mTOR pathway [493]. Resveratrol increases the autophagy and autophagy-mediated degradation of p62 in non-small lung adenocarcinoma A549 cells [494].

Various techniques have shown that resveratrol can induce autophagy in breast cancer stem cell-like cells [495]. Resveratrol inhibits tumor cell proliferation and induces apoptosis and autophagy in T acute lymphoblastic leukemia [496]. Silencing of SIRT1 expression inhibits autophagy by inhibiting the phosphorylation of p70RS6K and 4E-BP1, while molecular events are reversed in the presence of resveratrol [497].

A recent review provides more details about resveratrol activity in cancer [498]. Further clinical studies are important to fully evaluate the activity of resveratrol in the killing of tumor cells via autophagy.

Several studies have shown that non-flavonoid and flavonoids polyphenols including quercetin, apigenin, and epigallocatechin gallate (EGCG) can induce autophagy, both in vitro and in vivo. 
Members of the phytochemical flavonoid class of compounds, anthocyanins include cyanidin, delphinidin, pelargonidin, and petunidin. Naturally arising pigments, the various subgroups are defined by the flavylium B-ring. Delphinidin leads to notable LCE II lipidation, a cue necessary for autophagosome development [499].

A naturally occurring substance, hydroxycinnamates or E-[6'-(5'-hydroxypentyl) tricosyl]-4-hydroxy-3-methoxycinnamate (EHHM), is acquired from Livistona chinensis. It has been reported that autophagy enhances cell longevity in HCC cells administered EHHM; EHHM could therefore be a potentially efficacious strategy for treatment of this tumor [500].

When berberine is used in combination with lung cancer radiotherapy, in addition to inducing autophagy cell death in vitro and in vivo, it has also been shown to induce autophagy and apoptotic cell death in different hepatocellular carcinoma cells [501]. As evidenced by increased autophagosome formation, LC3B modification, and mitochondrial destruction, berberine enhances non-small cell lung cancer A549 cells' radiosensitivity undergo autophagy [502]. Berberine derivatives have been shown to induce autophagy, in addition to inhibiting the proliferation of different human colon cancer cells [486,503-506].

One of the major bioactive components in green tea is Epigallocatechin-3-gallate (EGCG) [507-509]. EGCG enhanced the effect of cisplatin and oxaliplatin-induced autophagy in human colorectal cancer cells [510]. EGCG increases the formation of autophagosomes, increasing lysosomal acidification, and stimulating autophagic flux in hepatic cells in vitro and in vivo [511]. Treatment of human hepatocellular carcinoma Hep3B cells with doxorubicin significantly increased a number of autophagic vesicles and levels of autophagic protein markers in tumor cells [512]. This effect resulted in $45 \%$ decrease in doxorubicin-induced cell death supporting pro-survival role for autophagy in these experimental conditions [512]. However, EGCG was found to inhibit autophagic signaling, and promoted cellular growth inhibition [512]. The doxorubicin-induced autophagy was blocked by the combination therapy with EGCG [512]. Rapamycin, an autophagic agonist, markedly inhibited the anticancer effect of doxorubicin or its combination with EGCG treatment [512]. Interestingly, EGCG was observed to increase non-apoptotic cell death in human hepatocellular carcinoma cells, cervical cancer cells, and mesothelioma cells $[513,514]$.

Curcumin is the major bioactive component extracted from Curcuma longa L., Curcuma zedoaria (Christm) Rose., Curcuma amada Roxb., and Curcuma petiolate $[353,358,477]$. Curcumin has been found to inhibit cell proliferation in several cancer types through inducing autophagy [515-521]. For example, in malignant glioma cells curcumin induced $\mathrm{G}_{2} / \mathrm{M}$ arrest and autophagy [480].

Antioxidant N-acetyl-L-cysteine (NAC) blocked the curcumin-induced molecular effects, suggesting that curcumin-induced ROS implicated in autophagosome development [521]. NAC also abolished curcumin-induced activation of ERK1/2 and p38MAPK [521].

The curcumin-induced autophagy was shown to be ROS-dependent [522]. Curcumin induced differentiation of glioma-initiating cells that are responsible for the initiation and recurrence of glioblastoma [523]. Curcumin was found to induce autophagy in these cells in vitro and in vivo [523]. Moreover, curcumin also suppressed tumor formation on intracranial implantation of glioma-initiating cells into mice [523]. Tetrahydrocurcumin, a major metabolite of curcumin, exhibited the antiproliferative effects on human promyelocytic leukemia HL-60 cells by increasing acidic vascular organelle formation specific for autophagy [524].

The survival rate against thioacetamide-induced HCC was observed to be increased by curcumin [473]. The combination of doxorubicin with curcumin caused autophagy stimulation. In the cells treated with this synergistic combination, high levels of the autophagosomes were detected [474].

Curcumin is the most extensively studied natural compound for the prevention and treatment of cancer [471]. However, curcumin has limited therapeutic effect due to its 
poor bioavailability and effectiveness. Therefore, many curcumin derivatives have been manufactured to evaluate its antitumor potency and those analogs might have an improved autophagy activity compared to curcumin and could provide a better activity toward the HCC cells [471].

Some of the Chinese medicinal herbs induce autophagy [353,366,473]. Fangchinoline triggered autophagy in some of human hepatocellular carcinoma cells. This herb is isolated from Fangji, Stephenia tetrandra S. Moore [525]. Blocking fangchinoline-induced autophagy process markedly modulated the apoptotic pathway [525]. Ginsenosides, major pharmacological active ingredients in Ginseng, induced cell death of tumor cells, thereby improving sensitivity of tumor cells to chemotherapy [329,526-530]. Ginsenosides Rg3 and $\mathrm{Rh} 2$ can inhibit cancer cell growth, while Rg3 is instrumental in combating tumor cell resistance to cancer chemotherapy $[329,531]$. A combination of Rg3 with docetaxel, paclitaxel, cisplatin, or doxorubicin enhanced the sensitivity of prostate cancer and human colon cells to chemotherapy [531]. The anticancer function of ginsenosides is associated with its ability to regulate autophagy in various human cancer cells [329,532-534]. Ginsenoside K activated an autophagy pathway mediate by increased autophagic flux [534].

Terpenoids are the largest class of natural compounds exhibiting multiple antitumor properties, especially due to their selectivity toward tumor and cancer stem cells (CSC) $[358,535,536]$. Sesquiterpene lactones are 15 - C terpenoids, such as parthenolide, artemisinin, and thapsigargin, were shown to be beneficial in cancer clinical trials [537-540]. Parthenolide was found to induce autophagy in the triple-negative breast cancer MDAMB231 cells [541]. Parthenolide treatment of human hepatocellular carcinoma HepG2 cells resulted in autophagic cell death [542].

Triptolide is a diterpenoid from the roots of Tripterygium wilfordii and was found to inhibit the proliferation of 60 US National Cancer Institute cancer cell lines [543,544]. Furthermore, its anticancer activities were confirmed in various animal models grafted with human tumors resulting in the development of several more water-soluble and less toxic derivatives that entered clinical trials [544]. Triptolide was shown to prevent human pancreatic tumor cell growth both in vitro and in vivo $[545,546]$. Betulinic acid is a triterpenoid isolated from the bark of the white birch tree that exhibited ant-tumor characteristcs against several cancer cells in vitro and in vivo $[547,548]$. Betulinic acid and its derivatives were found to decrease the phosphorylation of AKT and induce autophagic cell death in human glioblastoma cells [549]. However, in human multiple myeloma KM3 cells, betulinic acid treatment inhibited autophagy and induced apoptosis [550]. Betulinic acid was recently found to induce death in human cervical cancer HeLa cells, while caspase inhibitors and necrostatin-1 (NEC-1) blocked apoptosis and necroptosis, but not cell death in HeLa cells, implicating caspase-independent mechanisms of cell death in these cells [551].

Oridonin, a diterpenoid extracted from Rabdosia rubescent, promoted autophagy in L929 cells through p38 MAPK and nuclear factor kappa B (NF-kB) pathways [552-554]. Terpinen-4-ol was shown to induce autophagic and apoptotic cell death in human promyelocytic leukemic HL-60 cells through inducing the accumulation of LC3B-I/-II, ATG5, and $\mathrm{BECN1}$ proteins cytochrome $\mathrm{C}$ released from mitochondria, and decreasing $\mathrm{BCL}-\mathrm{xL}$ expression [555]. Celastrol, an active compound extracted from the root bark of Tripterygium wilfordii Hook F., exhibited $20 S$ proteasome inhibitor activity, while inducing apoptosis and autophagy in cancer cells [556-561]. Both apoptotic and autophagic pathways were found intertwined upon celastrol treatment, since inhibition of apoptosis enhanced autophagy, while suppression of autophagy diminished apoptosis in human osteosarcoma cells [557]. Celastrol could induce hypoxia-inducible factor (HIF)-1a protein accumulation leading to transcriptional activation of HIF-1 target genes [558,559].

Celastrol induced autophagy in human gastric cancer AGS and YCC-2 cells [561]. Moreover, gastric tumor burdens were reduced by celastrol administration in a mouse model with a grafted human gastric tumor [561]. 
Sulforaphane is found in cruciferous vegetables, such as broccoli, cabbage, cauliflower, and hoary weed [535]. Sulforaphane exhibited antiproliferative properties toward various human tumor cells via various molecular mechanisms [536,562-566]. Sulforaphane was shown to induce the formation of autophagosome-like structures as well as acidic vesicular organelles in human prostate cancer PC-3 cells [536]. Upon sulforaphane exposure, tumor cells showed LC3B-II puncta associated with autophagosomes [536]. Sulforaphane was also shown to disrupt the BCL-2/BECN1 interaction leading to the autophagic pathway initiated by liberated BECN1 [536]. Sulforaphane decreased the phosphorylated AKTSer473 level, and simultaneous treatment of sulforaphane with autophagy inhibitors, 3-MA, or chloroquine enhanced drug cytotoxicity and inhibited tumor cell proliferation $[536,566]$.

Recently, a novel alkaloid called Monanchocidin A (MonA) was isolated from the marine sponge Monanchora pulchra [567]. MonA exhibited cytotoxic activity towards human genitourinary cancer cells, including hormone-sensitive and castration-resistant prostate carcinoma cell lines, cisplatin-sensitive and -resistant germ cell tumor cell lines, and different bladder carcinoma cell lines. Whereas, nonmalignant cells were notably less susceptible [567]. MonA was found to induce autophagy and lysosomal membrane permeabilization in cancer cells [567]. Cryptotanshinone and dihydrotanshinone, two lipophilic tanshinones from a traditional Chinese medicine Salvia miltiorrhiza, were shown to induce autophagic flux and LC3B-II accumulation in multidrug-resistant colon cancer cells SW620 and Ad300 cells [568]. Cardamonin is derived from Alpinia katsumadai Hayata (Zingiberaceae) [568]. Cardamonin inhibited cell proliferation and enhanced autophagy in human colon colorectal carcinoma HCT-116 cells [569].

Cannabinoids promote autophagy-dependent apoptosis in melanoma cells [570]. Treatment with A (9)- Tetrahydrocannabinol (THC) activated autophagy, loss of cell viability, and apoptosis, whereas co-treatment with chloroquine prevented THC-induced cell death and autophagy in vitro [570].

Seriniquinone, isolated from a marine bacterium of the genus Serinicoccus, demonstrated potent anti-proliferative activity toward melanoma cell lines by activation of autophagocytosis, while targeting small protein, dermcidin [571]. Oblongifolin C (OC) is a natural small compound extracted from Garcinia yunnanensis $\mathrm{Hu}$. OC is a potent inhibitor of autophagic flux [572-574].

Plant lectins have been considered as possible antitumor drugs because of their ability to induce autophagic cell death [575-580]. Polygonatum odoratum lectin (POL), from traditional Chinese medicine herb, is a mannose-binding GNA-related lectin and was shown to exhibit apoptosis-inducing and anti-proliferative activities toward a variety of tumor cells [575]. POL could induce both autophagy and apoptosis in NSCLC A549 cells and human breast cancer MCF-7 cells [575,576].

Thymoquinone exposure resulted in caspase-independent, autophagic cell death in human LoVo colon cancer cells [581]. Honokiol (HNK), purified from the Magnolia officinalis bark, is a promising anticancer agent against prostate cancer cells in vitro and (PC-3 xenografts) in vivo [582]. Mollugin, a bioactive phytochemical isolated from Rubia cordifolia L., exhibited anticancer activity against various cancer cells [583].

Jujuboside B is a saponin from the Zizyphus jujuba var. spinosa seeds. Jujuboside B induced autophagy and apoptosis in human AGS and HCT-116 gastric adenocarcinoma cells in vitro and efficiently inhibited cancer growth in a nude mouse xenograft model bearing HCT-116 cells in vivo [584]. Moreover, jujuboside B induced autophagy indicated by the formation of cytoplasmic vacuoles and LC3B-I/II conversion [584]. Bafilomycin A1, which is an autophagy inhibitor, reduced the viability of jujuboside B-induced cells [584]. Feroniellin A (FERO), a novel furanocoumarin, was shown to induce autophagy as well as showing association with LC3B-I to LC3B-II conversion, induction of GFP-LC3B puncta, enhanced expression of BECN1, and ATG5, and inactivation of mTOR in etoposide-resistant human lung carcinoma A549RT-eto cells [585].

A polymethoxy flavonoid, nobiletin has been shown to inhibit cellular replication in human SKOV3/TAX cells through apoptosis and autophagic flux suppression. Compara- 
tively, via Akt signalling, the disturbed autophagic process activated nobiletin-induced apoptosis in this cell line. These data provided evidence to suggest that in human ovarian tumor cells, nobiletin can surmount multi-drug resistance by inhibiting autophagic disruption via Akt modulation [586]. EGFR and TKIs were the most significant for late-stage NSCLC.

Nevertheless, T790M mutation, which increases TKIs resistance generated by EGFR, has transpired to be a key difficulty in tumor therapy. An admixture of wogonin and icotinib was noted to surmount icotinib resistance arising via T709M [587]. An elevated population of intracellular autophagosomes, conversion of LC3B-I to LC3B-II, and Beclin-1 and phosphorylated mTOR expression amplification were identified following the combined administration of wogonin and icotinib. This implies that the two compounds have mutually potentiating influences on cell replication and could play a role in apoptosis and autophagy in EGFR T790M-mutated lung malignancy [587].

Derived from Sophoraflavescens Aiton, matrine is a major quinolizindine alkaloid [588]. The serial signal transduction giving rise to apoptosis from autophagy by triggering p53 has been the subject of discourse [589]. Metabolomic analysis of HepG2 cells administered matrine has revealed lipid droplet metabolites which form macroautophagy substrates partially responsible for immune response activation and apoptosis [590]. Matrine has also been shown to diminish glutathione (GSH) titres; a raised GSH concentration is implicated in tumor resistance to chemotherapy [591].

Mixing autophagy interventions with molecular targeted treatment is thought to offer a positive therapeutic approach to HCC [592]. Data amassed from the last twenty years have emphasized the significance of autophagy in a spectrum of human pathologies. Influencing this process by targeting certain modulators in the core autophagy pathways could therefore impact various pathophysiologies. Malignancies exhibit heightened and diminished autophagic cues, consistent with their cancer-suppressing and promoting characteristics during tumorigenesis. In order to design de novo drugs, recognition of key targets of the autophagic process is essential.

Polyphenols have a distinct capacity to inhibit cell replication and to initiate apoptosis or autophagy in HCC; this ability has drawn attention to their possible use for targeted treatments. In brief, polyphenols, e.g., apigenin, oroxylin A, and resveratrol, may act as inhibitors for the PI3K/Akt/mTOR signalling mechanism. LC3 II evolution, and thus autophagy inducement, is contributed to by luteolin, isoorientin, quercetin, kaempferols, curcumin, adriamycin (doxorubicin) with curcumin, EGCG, EHHM, delphinidin, EF25-(GSH) 2 , oroxylin A, resveratrol, and kaempferols. mTOR signalling pathway phosphorylation may be suppressed by myricetin. Autophagy could be instigated by galangin via the TGF-p receptor/Smad. Safeguarding autophagy linked with the negative modulation of CD147 and ER stress could be provoked by baicalein. Wogonin, in combination with sorafenib, WZ35, and tangeretin has anti-HCC effects mediated through autophagy inhibition.

Multiple biological properties of the naturally occurring polyphenols in the diet encompass antitumor and autophagy-enhancing influences. Research has demonstrated that polyphenolics enact their anti-HCC activities through interference with the autophagic process, e.g., activation of Beclin-1, Atg5, Atg7, Atg9, Atg12, LC3-II, and SQSTM1, together with the modulation of PI3K/Akt/mTOR, PTEN, P38/PPAR-a, JNK/Bcl-2, ER stress, p62, p53-dependent, TGF-p receptor/Smad signaling, and YAP. These data suggest that they are possible agents with multiple modes of action against HCC, which is a catastrophic pathology.

Key factors to take into account with pharmaceutical agents sanctioned by the FDA, e.g., sorafenib, are their adverse event and strength profiles, as these indicate potentially serious unwanted side effects, such as liver toxicity, inflammation, bleeding, fistulas, rashes, high blood pressure, ischemia, and wound reparation issues. Similarly, outcome data on HCC therapies in individuals with advanced cirrhosis are not accessible. Thus, a preferable option would be to couple autophagy-modulating hepatoprotective polyphenolic com- 
pounds, with favorable safety statistics, with anti-HCC agents sanctioned by the FDA in order to offer new treatment approaches encompassing autophagy modulation. Furthermore, the development of de novo therapies for HCC should include such polyphenols in their research.

The development of resistance to pharmaceutical agents decreases their therapeutic impact. As autophagy contributes to cancer advancement, influencing this process using naturally arising substances is a potentially encouraging approach to combat multidrug resistance in malignant cells. However, the lack of testing in in vivo models, therapeutic protocols, and evaluations of phytochemical toxicity weakens the published data from single studies and makes it challenging to predict the effectiveness of substances derived from plants in the therapy of specific forms of neoplasia.

\subsection{Targeting Oncosis}

Oncosis is described by cell lysis and rapid cell and organelle swelling, in addition to membrane permeability. Oncosis is associated with intercellular events involving swelling of the mitochondria, depletion of adenosine triphosphate (ATP), failure of calcium ion homeostasis, activation of certain proteases (such as cathepsins and calpains), disruption of lysosome, and finally rupture of the plasma membrane [265,593].

In addition to chemotherapy, radiation, genetic, or immune therapeutic strategies as well as combinatorial approaches, natural antitumor products with promising safety and efficacy are setting an important stage for the new anticancer treatments [288,329334,450,451,594,595] (Table 2).

Artemisinin, which was extracted and separated from Artemisia annua L. (sweet wormwood), has been used as one of the well-known traditional Chinese medicines for many years in the treatment of fevers and chills [596]. In pancreatic cancer and renal cell carcinoma, it has been shown that artemisinin induced oncosis-like cell death [597]. The former cell death occurred with the morphotype characteristics of oncosis, while the latter was via the generation of reactive oxygen species (ROS) and the depletion of ATP [597-599]. In gastric cancer, artemisinin stimulated cell oncosis by reducing the expression of Vascular endothelial growth factor (VEGF) and increasing the amount of calcium and the expression of calpain-2 [599,600].

The exact mechanism of action of artemisinin is still controversial, and the target of action has not been completely revealed. Current research shows that artemisinin uses multi-approaches and multi-links to influence the tumor progression. Both apoptotic and non-apoptotic cell death are involved in the anticancer activity of artemisinin. Furthermore, artemisinin affects cancer metabolism and immunosuppression. However, the related literature is still limited, and more in-depth research into these aspects is required.

\subsection{Targeting Methuosis}

One of the most recent forms of non-apoptotic cell death is methuosis. The name of methuosis, which is derived from the Greek 'methuo' and means 'to drink to intoxication', was chosen because the most prominent characteristic in cells undergoing this phenotype of death is the accumulation of large fluid-filled cytoplasmic vacuoles that originate from macropinosomes [601-603].

Macropinocytosis is defined as a non-selective liquid-phase endocytic pathway for the extracellular substances' uptake [604]. Macropinocytosis was first recognized in 1931, and the used term was pinocytosis, or cell-drinking [605]. Then, in 1986-1992, the stimulation of membrane ruffling and fluid-phase pinocytosis were described $[606,607]$ and a review about macropinocytosis was published in 1995 [608]. Later, it was found that abnormal expression of the RAS genes in glioma cells and gastric cancer would cause cellular degeneration in addition to vacuolization [609], which belongs to micropinocytosis. Eventually, this led to a new distinct phenotype of cell death. It was discovered that abnormal genetic manipulations as well as trace amounts of certain drugs can stimulate methuosis in cancer cells [610-612]. 
Recently, the relationship between tumors and macropinocytosis has attracted more attention as it might has a great research value for tumor survival and treatment [604]. Accordingly, several researchers are interested in macropinocytosis as a new target for cancer treatment in addition to its potential for antitumor drugs delivery and the design of antitumor drugs that can induce methuosis or abrogate the process of macropinocytosis have been reported. However, some challenges and queries exist about the research in this field.

Firstly, it is not clearly confirmed whether methuosis represents a recent unique phenotype of controlling cell death or whether it is just a subtype of oncosis or necrosis [601,613]. Additionally, the molecular mechanisms related to methuosis remain unclear despite the known importance of RAS genes and more detailed and specific evidence linking macropinocytosis directly to cell death is lacking. Indeed, there are more stimulants of methuosis waiting to be discovered for clinical use.

Additionally, macropinocytosis can both enhance cancer survival and has detrimental effects on cancers [614-625]. This makes methuosis different from classical apoptosis and other non-apoptosis death forms. Perhaps this means that macropinocytosis has a "threshold" between enhancing cancer cells survival and death. However, more studies are needed to know what exactly this "threshold".

The understanding of molecular pathways involved in non-apoptotic cell death induced by natural anticancer drugs would assist in exploiting novel molecular targets of plant-derived compounds necessary to advance safer and effective anticancer therapeutics allowing to circumvent cancer drug resistance [293,334,341,449,480,503,507,524, 610,626-632]. The success with the natural product shikonin, which was able to induce multiple cell death mechanisms, supports the notion that natural compounds could bypass specific drug resistance developed by tumor cells using simultaneous activation of multiple death pathways [293,334,341,449,480,503,507,524,610,626-632]. Therefore, a reasonable combination of several cell death inducers that complement each other will maximize their efficacy while minimizing their side effects $[293,334,341,449,480,503,507$, 524,610,626-632]. Although in vitro studies have shown that natural compounds have a strong ability to induce non-apoptotic death of tumor cells, more in vivo studies are needed to strengthen this concept before entering clinical applications [610,629-633]. Figure 3 summarizes the main natural products and their mechanism of action in triggering non-apoptotic cell death. 

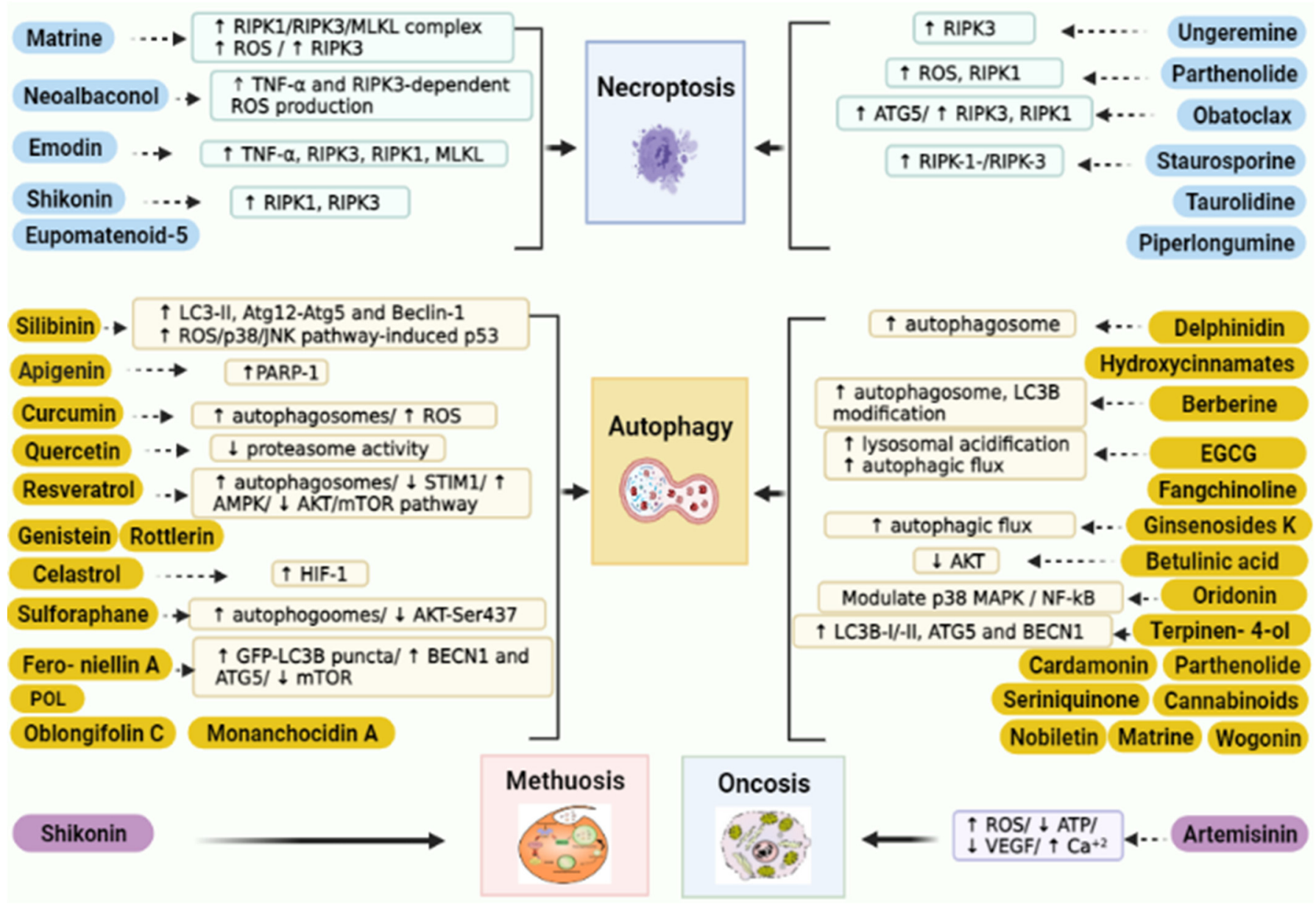

Figure 3. Natural products mechanism of action in promoting non-apoptotic cell death in cancer cells. RIPK1, receptorinteracting serine/threonine-protein kinase 1; MLKL, mixed lineage kinase domain-like; ROS, reactive species; TNF- $\alpha$, tumor necrosis factor; ATG5, autophagy related 5; LC-II, light chain 2; STIM1, stromal interaction molecule 1; AKT, protein kinase B; mTOR, mammalian target of rapamycin; AMPK, AMP-activated protein kinase; HIF-1, hypoxia-inducible factor-1; BECN1, beclin1; NF-kB, nuclear factor kappa B.

Table 2. A list of natural compounds with an effect on non-apoptosis cell death in tumor cells.

\begin{tabular}{ccc}
\hline Compound Name & Target & Reference \\
\hline Matrine & Necroptosis & {$[344]$} \\
\hline Neoalbaconol & Necroptosis & {$[345]$} \\
\hline Shikonin & Necroptosis & {$[293,322,334,341,346,449,480$,} \\
& Autophagy & $503,507,524,610,626-632]$ \\
\hline Emodin & Necroptosis & {$[348]$} \\
\hline Ungeremine & Necroptosis & {$[349]$} \\
\hline Staurosporine & Necroptosis & {$[362-364]$} \\
\hline Obatoclax & Necroptosis & {$[365-367]$} \\
\hline Piperlongumine & Necroptosis & {$[369,370]$} \\
\hline Eupomatenoid-5 & Necroptosis & {$[371]$} \\
\hline Rottlerin & Autophagy & {$[456,634]$} \\
\hline Genistein & Autophagy & {$[635,636]$} \\
\hline
\end{tabular}


Table 2. Cont.

\begin{tabular}{|c|c|c|}
\hline Compound Name & Target & Reference \\
\hline Quercetin & Autophagy & {$[483,611]$} \\
\hline Resveratrol & Autophagy & $\begin{array}{c}{[458,480,492,494,612,637-647]} \\
{[458,480,494,612,637-641]}\end{array}$ \\
\hline Anthocyanins & Autophagy & [499] \\
\hline Hydroxycinnamates & Autophagy & [500] \\
\hline Berberine & Autophagy & $\begin{array}{c}{[486,501,503-} \\
505,594,595,648,649] \\
\end{array}$ \\
\hline Epigallocatechin-3-gallate & Autophagy & {$[534,538,539,650]$} \\
\hline Curcumin & Autophagy & {$[515,517-520,522,523,651-654]$} \\
\hline Fangchinoline & Autophagy & [525] \\
\hline Ginsenosides & Autophagy & {$[329,526-533]$} \\
\hline Terpenoids & Autophagy & {$[358,535,536]$} \\
\hline Triptolide & Autophagy & {$[568,570,655]$} \\
\hline Betulinic acid & Autophagy & {$[547,548]$} \\
\hline Oridonin & Autophagy & {$[558,559]$} \\
\hline Celastrol & Autophagy & {$[561,656]$} \\
\hline Sulforaphane & Autophagy & {$[581,585,587,592]$} \\
\hline Monanchocidin A & Autophagy & [567] \\
\hline Cryptotanshinone & Autophagy & {$[568,569]$} \\
\hline dihydrotanshinone & Autophagy & {$[568,569]$} \\
\hline Cannabinoids & Autophagy & [570] \\
\hline Seriniquinone, & Autophagy & [571] \\
\hline Oblongifolin C & Autophagy & [572-574] \\
\hline Polygonatum odoratum lectin & Autophagy & {$[575,576]$} \\
\hline Honokiol & Autophagy & {$[582,583]$} \\
\hline Jujuboside B & Autophagy & {$[607,609,613]$} \\
\hline Nobiletin & Autophagy & [587] \\
\hline Matrine & Autophagy & [589-591] \\
\hline Parthenolide & Autophagy & {$[565,657,658]$} \\
\hline Allicin & Autophagy & {$[659,660]$} \\
\hline Citreoviridin & Autophagy & [659-662] \\
\hline 7-hydroxydehydronuciferine & Autophagy & [663] \\
\hline Glycyrrhetinic acid & Autophagy & [664] \\
\hline Honokiol & Autophagy & [579] \\
\hline Artemisinin & Oncosis & {$[597,600]$} \\
\hline Matrine & Necroptosis & [368] \\
\hline Neoalbaconol & Necroptosis & {$[369]$} \\
\hline Shikonin & $\begin{array}{l}\text { Necroptosis } \\
\text { Autophagy }\end{array}$ & $\begin{array}{l}{[317,346,358,365,370,473,527} \\
532,551,652-654,656,659-663]\end{array}$ \\
\hline Emodin & Necroptosis & [372] \\
\hline Ungeremine & Necroptosis & [373] \\
\hline Staurosporine & Necroptosis & [386-388] \\
\hline
\end{tabular}


Table 2. Cont.

\begin{tabular}{|c|c|c|}
\hline Compound Name & Target & Reference \\
\hline Obatoclax & Necroptosis & [389-391] \\
\hline Piperlongumine & Necroptosis & {$[393,394]$} \\
\hline Eupomatenoid-5 & Necroptosis & [395] \\
\hline Rottlerin & Autophagy & {$[480,665]$} \\
\hline Genistein & Autophagy & {$[666,667]$} \\
\hline Quercetin & Autophagy & {$[507,668]$} \\
\hline Resveratrol & Autophagy & $\begin{array}{c}{[482,516,518,653,669-680]} \\
{[482,518,653,669-674]}\end{array}$ \\
\hline Anthocyanins & Autophagy & [523] \\
\hline Hydroxycinnamates & Autophagy & [524] \\
\hline Berberine & Autophagy & {$[525,527-530,623,624,681,682]$} \\
\hline Epigallocatechin-3-gallate & Autophagy & {$[534,538,539,650]$} \\
\hline Curcumin & Autophagy & {$[542,544-547,549,550,683-686]$} \\
\hline Fangchinoline & Autophagy & [552] \\
\hline Ginsenosides & Autophagy & {$[353,553-560]$} \\
\hline Terpenoids & Autophagy & {$[358,535,536]$} \\
\hline Triptolide & Autophagy & {$[568,570,655]$} \\
\hline Betulinic acid & Autophagy & {$[571,572]$} \\
\hline Oridonin & Autophagy & {$[582,583]$} \\
\hline Celastrol & Autophagy & {$[585,687]$} \\
\hline Sulforaphane & Autophagy & {$[581,585,587,592]$} \\
\hline Monanchocidin A & Autophagy & [593] \\
\hline Cryptotanshinone & Autophagy & {$[265,594]$} \\
\hline dihydrotanshinone & Autophagy & {$[265,594]$} \\
\hline Cannabinoids & Autophagy & [595] \\
\hline Seriniquinone, & Autophagy & [596] \\
\hline Oblongifolin C & Autophagy & [597-599] \\
\hline Polygonatum odoratum lectin & Autophagy & {$[600,601]$} \\
\hline Honokiol & Autophagy & {$[607,608]$} \\
\hline Jujuboside B & Autophagy & {$[607,609,613]$} \\
\hline Nobiletin & Autophagy & [615] \\
\hline Matrine & Autophagy & [617-619] \\
\hline Parthenolide & Autophagy & {$[565,657,658]$} \\
\hline Allicin & Autophagy & {$[688,689]$} \\
\hline Citreoviridin & Autophagy & [688-691] \\
\hline 7-hydroxydehydronuciferine & Autophagy & [692] \\
\hline Glycyrrhetinic acid & Autophagy & [693] \\
\hline Honokiol & Autophagy & [604] \\
\hline Artemisinin & Oncosis & {$[626,628]$} \\
\hline
\end{tabular}




\section{Clinical Studies}

Although most antitumors reduce the size of tumors significantly [665], they fail to remove them completely. As a result, the tumor resists treatment and relapses. The main reason for cancer treatment failure by chemotherapy is the emergence of tumor cell death resistance to drugs during cancer progression, thus representing a central issue in chemotherapeutic approaches to this tumor. Multidrug resistance (MDR) is one of the major clinical challenges in malignancy treatment and compromises the effectiveness of conventional antitumor drugs.

The ability of natural products and their derivatives to prevent, inhibit, and reverse the progression of cancer has been clinically studied. Surveys indicate that approximately $80 \%$ of cancer patients use natural products in combination with classic anticancer drugs [666]. This shows that many cancer patients are very interested in using natural products as nutritional supplements or complementary or alternative medicines. They hope that these natural products can significantly reduce the side effects caused by anticancer drugs, enhance the immune response, and enhance the effectiveness of anticancer drugs. Some people believe that they will actually prevent or reverse the progression of cancer.

More and more evidence has shown that natural compounds are highly specific to tumor cells and have minimal adverse effects on normal neighboring cells; therefore, they bring great hopes for future anticancer therapies $[334,633]$. It is reported that more than 3000 plant species can treat cancer. Thus far, about 30 plant-derived compounds have been isolated and tested in cancer clinical trials [334] and are used currently in clinical practice exhibiting advantageous results, with some exhibiting serious toxic side effects.

The principal objectives for combination therapies encompassing prolongation of survival rates and enhancing life quality include mitigating the cytotoxic adverse event profiles of pharmaceutical agents and simultaneously diminishing tumor resistance and unwanted drug effects.

Natural compounds such as flavonoids, sesquiterpenes, alkaloids, diterpenoids, and saponins, in addition to polyphenolic compounds, to overcome drug resistance $[667,668]$ are substituted or applied in combination with existing drugs. The principal objectives for combination therapies encompassing prolongation of survival rates and enhancing life quality include mitigating the cytotoxic adverse event profiles of pharmaceutical agents and simultaneously diminishing tumor resistance and unwanted drug effects. These natural compounds are known to have anticancer effects and can both kill cancer cells and restore drug sensitivity.

Although sufficient data were available from clinical trials conducted on animals to prove the efficacy of resveratrol with respect to tumor therapy, few in vitro clinical studies have been conducted in human cells. Thus, there is a dearth of results relating to human trials that assess the effectiveness of resveratrol in cancer resistance treatment. The data exhibited unpredictable outcomes with respect to the use of resveratrol because the majority of these clinical trials used different doses and routes of administration with a small sample size of patients [498].

Resveratrol can efficiently exhibit its antitumor effects in combination with other chemotherapeutic agents in addition of having an excellent safety profile. A number of important challenges need to be considered before bringing resveratrol to the bedside owing to its rapid metabolism giving rise to limited bioavailability in humans [498].

The role of melatonin in cancer treatment and prevention has been widely studied, and numerous experimental studies proved the antitumor effect of melatonin against many types of cancers. The combination of conventional anticancer therapies with melatonin showed promising results through reinforcing the therapeutic effects of these therapies [669]. Overall, the high safety profile, diverse mechanisms of action, and high efficiency of melatonin support its use in cancer prevention and treatment [669].

It has been proposed that melatonin's benefit in mitigating the toxic effects of chemotherapy and its association with aberrant mitochondrial function should be explored using double-blind, placebo-controlled trials. It can be expected that a plethora of 
information will emerge over the next ten years relating to the way in which melatonin exerts a positive effect in conjunction with chemotherapeutic agents [670]. The development of resistance to therapy, together with the occurrence of tumor spread, means that the investigation of de novo modes of treatment for malignancies is essential.

It is well established that therapy for individuals presenting with glioblastomas is complex; curative surgery is nearly impossible, and the majority of tumors exhibit a high recurrence rate despite treatment with radiation and chemotherapy [671]. Thus, several workers have concentrated on the development of de novo adjuvant treatment approaches, favoring natural products in order to offer anticancer agents that are suitable for the clinical practice. A number of studies have documented the characteristics of melatonin with respect to glioblastomas. Melatonin has well-known anti-oxidant actions, and its antitumor effects are becoming acknowledged. It therefore has potential to thwart the resistance to numerous anticancer agents that plagues treatment of glioblastomas [671-673]. Melatonin was reported as a candidate to overcome the multi-drug resistance glioblastoma treatment $[672,694,695]$. Additional work is required to design novel molecular products, combination treatments, and optimal dosing regimens. Although a few studies have reported anticancer actions of melatonin in relation to glioblastoma in vitro, as yet, few animal models have been published, and there is scant literature available on this subject in humans.

Lissoni et al. published a study investigating treatment with an admixture of melatonin and aloe vera [674]. The purpose of this trial was to determine whether these two products could act in synergy to enhance the anticancer characteristics of melatonin. Fifty patients with malignancy, including patients who had developed resistance to chemotherapy, radiation, and hormone treatments, or who were unable to tolerate chemical anticancer agents, were recruited for the study. Eight weeks after therapy commencement, no effect on lesion regression was seen in the cohort only receiving melatonin. In the group taking aloe vera and melatonin, $2 / 24$ patients ( $8 \%$ ) exhibited a partial response. The safety profile for melatonin was benign [674].

The data relating to the utilization of melatonin as an adjunct to chemotherapy are encouraging, both in terms of augmenting the effectiveness of therapy and mitigating adverse event profiles $[672,675]$. However, clinical studies that have investigated the clinical efficacy of melatonin in conjunction with other forms of treatment in patients with neoplasia, excluding glioblastoma, have usually been performed outside evidence-based recommendations following lack of success with conventional therapy and a guarded life expectancy.

Traditional Chinese Medicine (TCM) uses a combinatorial method of two or more agents to achieve a synergistic effect [676]. TCM has been utilized worldwide as a complementary or an alternative medicine and has long been used to treat cancer in China [677-682]. Examples of TCM's main components include alkaloids, flavonoids, and saponins. Numerous natural products originating from TCM can reverse multidrug resistance [650]. Research has shown that flavonoids reverse multi-drug resistance [683].

Curcumin is a common term used for a mixture of curcuminoids that are purified from the Indian spice turmeric powder, mainly comprised of curcumin (curcumin I), demethoxycurcumin (curcumin II), and bisdemothoxycurcumin (curcumin III) [684]. Curcumin is a traditional medicine and the main curcuminoid of Curcuma longa $[685,686]$.

Curcuminoids are known to have many biological activities, including anti-inflammatory [655,687], anticancer [657], and antiviral properties [658,688]. Moreover, both curcumin and its major metabolite tertrahydrocurcumin were found to restore drug sensitivity in tumor cells overexpressing the MOR-linked ABC transporters Pgp [684,689], MRP1 $[689,690]$, and ABCG2 $[689,691]$ by directly inhibiting their functions. More recently, curcumin was found to be active against MDR tumors in mice as well [692]. Considering its inhibitory effect on multiple $A B C$ drug transporters and its many beneficiary biological properties, it is not surprising that curcumin has become one of the most exciting natural product modulators in recent years. 
Clinically, poor bioavailability is the one major problem with using curcumin. The levels of curcumin in plasma and tissues remain low after oral consumption, reported to be in the range of nanomolars and picomolars, respectively [693]. Curcumin is lipophilic and very insoluble in nature, and is also rapidly metabolized in the intestine and excreted in the urine, which means that high doses of curcumin must be consumed for it to be biologically relevant and effective [696]. For instance, in one study, the level of curcumin was only barely detectable in human plasma even after a dose of curcumin as high as $12.0 \mathrm{~g}$ [697]. Therefore, several approaches have been investigated to improve the delivery of curcumin in the human system [698], including the use of liposomal curcumin [699], curcumin nanoparticles [700,701], curcumin phospholipid complex or structural analogues of curcumin [702], or the use of a combination of curcumin and piperine. Piperine has been shown to block the metabolism of curcumin by P450 A3 and by other hepatic and intestinal pathways involved in glucuronidation of this compound [703]. In addition, it was observed that piperine increased the bioavailability of orally given curcumin in both rats and humans with no adverse effect [703]. Thus, it should be useful to test whether the combination of curcumin and piperine improves the bioavailability of co-administered antitumor drugs in cancer patients.

Clinical trials offer the opportunity to verify and to identify the impact, side effects, and pharmacokinetics of therapeutic agents. Since curcumin's bioavailability is low, many curcumin formulations have been manufactured and have undergone testing in clinical trials $[694,695,704]$. A phase I clinical study was performed in order to establish the safety and pharmacokinetic profiles of theracurmin in individuals with malignancy of the pancreas and biliary tract in whom conventional chemotherapeutic agents had been unsuccessful [695]. Daily theracurmin, in combination with chemotherapy founded on gemcitabine, was administered. No additional side effects or rise in their incidence were reported. A phase II pilot study showed promising data for the admixture of docetaxel/prednisone and curcumin in individuals with prostate tumors resistant to orchidectomy. In 59\% and $40 \%$, respectively, either complete or some degree of prostate-specific antigen response was noted. This work offered further data indicating a high rate of response and acceptability for curcumin administration during treatment for malignancy [705]. Despite the optimistic published articles and clinical trials regarding curcumin's potential effectiveness against cancer, there is evidence to show that curcumin has no therapeutic benefits [706]. However, researchers still think that because of suggestive trends in trial results and because curcumin can interact with many proteins, there is still justification for further study [707]. The antitumor activity of curcumin remains unconfirmed until better experiments are carried out.

More importantly, both phase I and II clinical studies with curcumin have been carried out and showed some encouraging results. Despite its poor bioavailability, phase I studies indicated that curcumin is well tolerated [708] and provided substantial improvement in patients with advanced colorectal cancer when treated with curcumin (360-500 mg) [693], with minimal drug-curcumin interactions [709]. Likewise, phase II studies showed treatment with curcumin can improve the clinical outcome in patients with advanced pancreatic cancer [710]. These clinical studies suggest that it would be worthwhile to test curcumin as an adjuvant along with traditional chemotherapy drugs to overcome MDR in cancer patients.

Among all natural product modulators, the most well-studied and well-known are flavonoids, which include flavonols, flavones, isoflavones, flavanols, flavanolols, flavanones, and chalcones [711]. Typically, each person consumes a substantial amount of flavonoids per day from fruits, vegetables, food supplements, and tea. They are known to have many prominent health benefits [712,713], including anticancer properties [714-716]. In terms of MDR, flavonoids have been studied and characterized extensively by many research groups to determine their capability to restore drug sensitivity in MDR tumor cells [717-720].

Artemisinin possesses some advantages, including less susceptibility to resistance, that makes it worthy of development as a novel anticancer agent. In Pubmed, there are only 
three studies in the last three years. Some previous clinical studies before 2019 have been comprehensively reviewed by Efferth and Zhang et al. [721,722]. Despite using different modes of artemisinin administration for different cancers, no solidly concluded results can be seen yet.

Sanctioned by the State FDA of China, the compound Kushen Injection (CKI) has been utilized as an adjunctive therapy to Western antitumor medication for various forms of malignancy [723]. It comprises alkaloids, flavonoids, saccharides, and organic acids [724] and is derived from the medicinal plants, Radix Sophorae flavescentis and Rhizoma Smilacis Glabrae [725]. CKI is believed to be efficacious in preventing metastasis and overcoming multi-drug resistance [725]. However, to date there are no studies to this effect in the literature available in English; any existing research presents few convincing results. Thus, in vivo work and the clinical pertinence of CKI requires additional study in order to determine the efficacy of any chemosensitizing properties.

Polyphenolic natural products, such as Ellagic Acid and Schisandrins, represent a chemically unique class of molecules as potential anticancer agents to overcome multidrug resistance in cancer [93]. However, clinical studies to support this benefit are required.

Epigallocatechin gallate (EGCG) is one of the major bioactive components in green tea. EGCG enhances the effect of cisplatin and oxaliplatin-induced resistance of cancer cells and exerts synergistic effects with anticancer agents. In addition to cisplatin and oxaliplatin, these agents include temozolomide, doxorubicin, resveratrol, vardenafil, erlotinib, and curcumin [726-728].

In order to appraise the acceptability, pharmacokinetics, and effectiveness of EGCG in humans for tumor therapy, clinical studies are in progress. A phase I clinical trial evaluating therapy for radiation dermatitis in patients with breast neoplasia studied concurrent radiotherapy and EGCG solution. The highest dose of 660 pM of EGCG was without significant side effects [729], and the solution was deemed efficacious for the therapy of radiation dermatitis. EGCG was also appraised in a phase II clinical study to explore its advantages in acute radiation-induced oesophagitis in individuals with stage 3 lung tumors. Oral EGCG delivery was proven to be useful; phase III studies are expected to follow [730].

Gambogic acid (GA) is one of the main compounds derived from the gambogic resin exuded from a plant of the genus Garcinia [731]. It has a variety of biological activities, including anticancer properties [732]. The combination of GA and other anticancer agents has been widely used to improve the therapeutic effectiveness against various tumors [732-735]. Cisplatin resistance, which is a main clinical challenge in the treatment of lung cancer, can be decreased in human NSCLC cisplatin-resistant A549/ DDP cells by combining cisplatin and GA [734].

To asses the safety and effectiveness of GA in patients with advanced malignant tumors, different doses have been compared in a phase IIa clinical trial [736]. GA is safe at a dose of $45 \mathrm{mg} / \mathrm{m}^{2}$. Patients taking GA on days 1-5 within a two-week cycle showed a higher rate of disease control, with only grade I and II adverse reactions. A phase IIb clinical trial with a larger sample size of participants would be required to better investigate the safety and efficacy of GA.

A major limitation in all of the clinical studies has been the identification of appropriate pharmacodynamic biomarkers evaluating changes in autophagy.

Autophagosomal configurations can act as scaffolds to initiate apoptosis [365] and necroptosis [365,737], and so their accrual may enhance these cues within some contexts. If this notion were true, it may be preferable to inhibit autophagosomal breakdown with a lysosomal inhibitor instead of suppressing autophagosome production which may avoid malignant cell eradication. Lastly, the issue of autophagy inducers to circumvent oncogenesis requires addressing. It has been postulated that enhancing autophagy may hinder the onset of neoplasia by restricting genomic mutations, fostering oncogene-produced quiescence and diminishing cancer-induced inflammation [738]. This is therefore a complicated problem owing to the interplay of autophagy with various genetic contexts, e.g., the 
mutations of p53 recognized in tumors of the pancreas [739] and breast [740] where the reaction to autophagy initiation may be impacted by the sequencing of p53, thus creating a pro- or anti-carcinogenic effect.

Contemporary clinical trial designs frequently permit specimen gathering from malignancies, together with serum pre and post therapy. These may assist in the generation of improved bioindicators to act as pharmacodynamic markers for the effectiveness of autophagy inhibitors and to enhance patient selection for this therapy type. If enhanced clinical trials are added to in-depth molecular and cellular appraisal in order to comprehend the pathways underpinning the setting-reliant influence of autophagy on malignancy, a more logical foundation to inform judgements about when and in which trajectory autophagy could be influenced during tumor treatment could be developed. As changes in autophagy are inevitable during malignancy and such alterations will impact cancer progression, turning a blind eye to the issue is a poor choice. It is preferable to comprehend the biology and then use that information in effectively designed clinical studies.

The final objective of laboratory tests is to attain clinical usage. In clinical trials, subjects are typically split into two cohorts, i.e., controls administered traditional chemotherapy and the intervention arm who are additionally given natural products. The impact of Fritillariae thunbergia was evaluated in 90 individuals with acute leukemia [741]; compared with controls, individuals in the intervention arm exhibited a smaller population of leukemic cells within the bone marrow, diminished MDR1 protein titres, and a lower remission rate. The same substance was appraised in 30 individuals with acute leukemia with elevated MDR1 expression [742]. The intervention arm subjects had three-fold reduced MDR1 protein concentrations, an increased response rate to therapy, i.e., $55 \%$ as opposed to $20 \%$, and a smaller bone marrow proportion of leukemic cells, i.e., $26 \%$ compared to $50 \%$. A total of 36 individuals with acute leukemia underwent measurement of bone marrow cell mRNA titres of MRP and `2M using real-time PCR. An MRP/^2M parameter $\geq 0.3$ was deemed MRP positive [743]. Those subjects receiving a 15-day course of $120 \mathrm{mg}$ daily intravenous ligustrazine had a greater likelihood (45.5\%) of becoming negative for MRP than controls (7.1\%) and to have a reduced fraction of bone marrow leukemic cells, i.e., $21.4 \%$ as opposed to $55.6 \%$.

Inhibitors or modulators derived from natural resources are occasionally termed 'fourth-generation inhibitors'. Such substances offer a spectrum of de novo chemical scaffolds that are apposite for the creation of new agents. It can be anticipated that scientists appreciate the role of screening for novel natural compounds that exhibit these properties, as they are more likely to have a positive outcome than many existing products. There is an enormous range of resources that could be used; biologically active constituents are currently derived from vegetation, fungi, and even sea life, following which they are purified and studied in depth. An advantage is that these natural substances are typically of minimal toxicity and produce few side effects.

Individuals with solid tumors [744] and disseminated breast malignancy [745,746] found artemisinin to be tolerable and non-toxic. Nevertheless, prompts for ongoing surveillance during artemisinin prescription to record adverse effects should be contemplated particularly when it is utilized in high dosages. If appropriate, pharmaceutical agents used to avoid side effects should be given together. Furthermore, despite the fact that in patients with malignancy it can be challenging to distinguish whether adverse incidents are a result of pathology or medication, recent data indicate that such reports may have been associated with artemisinin; this should be evaluated in further clinical studies. Certain variables, i.e., route of delivery, quantity, and course length of medication, affect safety and effectiveness, and this needs to be studied in depth. Lastly, the accessible data from the phase I clinical studies for artemisinin were restricted, and the patient population was low in number. Thus, more expansive clinical trials for phases II, III, and IV are required in order to offer more compelling data for the appropriateness of artemisinin in clinical cancer therapy. 
Although uncertainty remains, clinical modulation of autophagy in oncology is currently in progress [747] with most endeavors aimed towards autophagy suppression. In fact, a survey of the ClinicalTrials.gov website, entering the terms 'autophagy' and 'cancer', brought up over 50 studies which concentrated on autophagy inhibition and assessment to enhance clinical endpoints. In keeping with other sectors of tumor biology, e.g., the promise of the immune system to potentiate and to suppress carcinogenesis and advancement, the principal factor impacting positive outcomes from treatments relating to autophagy arises from the delineation of the way in which the autophagic process influences cancer onset and evolution.

Chemical biology methods and cell culture-based approaches are powerful but have limitations that can unintentionally introduce confusion or uncertainty conclusion. Consideration of these limitations may help avoid common pitfalls and, in the fullness of time, lead to useful reinterpretation of existing data. Thus, additional future studies using in vivo systems and better clinical trials for the clinical management of resistance to drug-induced tumor cell death are needed to determine the clinical effectiveness and safety of these natural compounds.

\section{Safety Aspects of Natural Products in Cancer Management}

While many natural products used for cancer are associated with minimal or no risk, this is not true for all such therapies. Potential toxicities include direct toxicity of natural products, indirect effects of natural products due to interactions with other medications, and also the risk to the patient who uses natural products to avoid or delay established, effective treatment in the management of cancer disease [748,749].

There are some potential side effects associated with commonly used natural products and other types of CAM [750,751]. For example, green tea can cause emesis and diarrhea, in addition to insomnia and confusion.

Many natural products are pharmacologically active, raising concerns about potential interactions with conventional therapy, both cytotoxic agents, and other medical therapies [752-755]. Many anticancer drugs are metabolized through the cytochrome p450 system. Thepolyphenols present in green tea suppress many cytochrome p450 enzymes, which are important in drug metabolism and induce other drug-metabolizing enzymes. Several components of green tea and green tea extract can antagonize the effectiveness of bortezomib by different interaction mechanisms while increasing the effect of medicine such as anthracycline, taxanes, and tamoxifen (CYP3A4 inhibition) [756]. Additionally, Essiac, which consists of multiple biologically active substances, can act synergistically with anticancer drugs by the cytotoxic or immunosuppressive activities of anthraquinones existing in this mixture or through its inhibition of CYP3A [757]. Ginkgo biloba and panax ginseng enhance the functional activity of many drug-metabolizing CYP family enzymes, and it is recommended to be avoided in patients receiving agents metabolized by CYP3A4 or CYP2C19 [758]. Curcuma, used in some types of cancers, can cause nausea, gastric irritation, diarrhea, and bleeding problems. Curcuma interacts with many drugs, mainly doxorubicin and cyclophosphamide [759].

Although not a direct "toxic" effect, the use of natural products may result in a significant delay in instituting conventional treatment that is of documented benefit for a specific condition [760]. As non-natural products, in some cases, are imagined to negate the benefit of the therapy, it is usually required that natural products alone be taken for the duration of the CAM treatment. This strategy of care can lead to the rejection of effective medical therapies [748]. Although the data are limited, there is an adverse impact of refusing/delaying standard treatments in favor of alternative therapies [761,762].

In a previous report, 258 patients diagnosed with nonmetastatic breast, prostate, lung, and colorectal cancer in the National Cancer Database between 2004 and 2013 who underwent alternative medicine treatment as the sole therapy (identified as those coded as "other unproven cancer treatments administered by nonmedical personnel") and who also did not receive conventional cancer treatment were compared with a matched cohort 
of 1032 patients who received conventional cancer therapy [762]. Patients who chose alternative therapy had higher refusal rates for surgery ( 70 versus 0.1 percent), radiation therapy (53 versus 22 percent), chemotherapy (34 versus 3 percent), and hormone therapy (34 versus 3 percent). Alternative medicine use was associated with worse five-year overall survival ( 82.2 versus 86.6 percent), while the use of alternative treatment was independently associated with a greater risk of death (hazard ratio [HR] 2.08, 95\% CI 1.50-2.90). These data indicate that the refusal of conventional cancer therapy was associated with the mortality risk. Important limitations of this study are its observational nature and the reliance on medical diagnosis coding at a single facility, which may have underascertained the use of conventional cancer treatment for patients who received treatment at a different facility or those who initially received alternative medicine prior to presenting to the facility that was reporting data.

The use of autophagy-related kinase inhibitors/activators may lead to unwanted and uncontrolled side effects, despite their potential therapeutic benefits in animal models. Considering the protective properties of autophagy on neurons, it is reasonable to enhance the brain specificity of autophagy-related therapies for neurodegenerative conditions. Different delivery approaches and photodynamic chemotherapy are proposed to attain the goal of organ specificity. Additionally, as known for the common kinase drugs, these autophagy-related kinase inhibitors/activators share the complications of target selectivity and resistance [763].

The lack of cancer cells' killing selectivity of thapsigargin prevent its direct use as an anticancer agent. To transport thapsigargin directly to cancer sites, some prodrugs have been developed. For example, G115 and G202 are prodrugs created by a conjugation of thapsigargin to substrates of proteolytic enzymes that are available only in tumor cells. Additionally, JQ-FT is an antileukemic prodrug established by a conjugation of a thapsigargin derivative and folic acid. These prodrugs represent an efficient way to overcome thapsigargin cytotoxicity and provide targeted cancer therapy [764].

\section{Conclusions}

Natural products are emerging as a promising source for effective anticancer agents. The numerous sources of these products cause high diversity in targets and mechanisms of action. Such diversity has encouraged scientists to consider natural products as therapies for drug resistance in cancer. Some natural products showed high potential to target drug resistance mechanisms in cancer and caused tumor regression. Many of these natural compounds were successfully considered as therapies in preclinical and clinical studies. However, the use of natural product as a standard therapy to treat drug resistance is still limited. Further studies are needed to explore the potential of natural products in combination therapies to overcome drug resistance. Future studies can focus on studying the possible synergistic effect of natural product combinations to target multiple biomarkers in drug resistance. Studies can also consider using natural products as adjuvant treatments to augment conventional anticancer therapies.

Author Contributions: Conceptualization, W.H.T.; methodology, A.R.A., M.B. and M.I.A.-T.; software, A.I.M.; validation, W.H.T. and A.I.M.; formal analysis, A.R.A., M.B. and M.I.A.-T.; investigation, A.R.A., M.B. and M.I.A.-T.; resources, W.H.T. and A.I.M.; data curation, A.R.A., M.B. and M.I.A.-T.; writing-original draft preparation, W.H.T. and A.I.M.; writing—review and editing, A.R.A., M.B. and M.I.A.-T. visualization, A.I.M.; supervision, W.H.T.; project administration, W.H.T.; funding acquisition, W.H.T. All authors have read and agreed to the published version of the manuscript.

Funding: This research received no external funding.

Institutional Review Board Statement: Not applicable.

Informed Consent Statement: Not applicable.

Data Availability Statement: Not applicable. 
Acknowledgments: The authors are grateful to the Applied Science Private University, Amman, Jordan, for the full financial support granted to this research (Grant No. DRGS-2020-2021-4).

Conflicts of Interest: The authors declare no conflict of interest.

\section{References}

1. Wang, X.; Zhang, H.; Chen, X. Drug resistance and combating drug resistance in cancer. Cancer Drug Resist. 2019, 2, 141-160. [CrossRef] [PubMed]

2. Rueff, J.; Rodrigues, A.S. Cancer drug resistance: A brief overview from a genetic viewpoint. Cancer Drug Resist. 2016, 1395, 1-18.

3. Nikolaou, M.; Pavlopoulou, A.; Georgakilas, A.G.; Kyrodimos, E. The challenge of drug resistance in cancer treatment: A current overview. Clin. Exp. Metastasis 2018, 35, 309-318. [CrossRef] [PubMed]

4. Gottesman, M.M. Mechanisms of cancer drug resistance. Annu. Rev. Med. 2002, 53, 615-627. [CrossRef] [PubMed]

5. Daniel, C.; Bell, C.; Burton, C.; Harguindey, S.; Reshkin, S.J.; Rauch, C. The role of proton dynamics in the development and maintenance of multidrug resistance in cancer. Biochim. Biophys. Acta 2013, 1832, 606-617. [CrossRef] [PubMed]

6. Perez-Tomas, R. Multidrug resistance: Retrospect and prospects in anti-cancer drug treatment. Curr. Med. Chem. 2006, 13, 1859-1876. [CrossRef]

7. Guo, Q.; Cao, H.; Qi, X.; Li, H.; Ye, P.; Wang, Z.; Wang, D.; Sun, M. Research progress in reversal of tumor multi-drug resistance via natural products. Anti-Cancer Agents Med. Chem. 2017, 17, 1466-1476. [CrossRef]

8. Quagliano, A.; Gopalakrishnapillai, A.; Barwe, S.P. Understanding the mechanisms by which epigenetic modifiers avert therapy resistance in cancer. Front. Oncol. 2020, 10, 992. [CrossRef]

9. Cragg, G.M.; Newman, D.J. Natural products: A continuing source of novel drug leads. Biochim. Biophys. Acta 2013, 1830, 3670-3695. [CrossRef]

10. Dutta, S.; Mahalanobish, S.; Saha, S.; Ghosh, S.; Sil, P.C. Natural products: An upcoming therapeutic approach to cancer. Food Chem. Toxicol. 2019, 128, 240-255. [CrossRef]

11. Atanasov, A.G.; Waltenberger, B.; Pferschy-Wenzig, E.-M.; Linder, T.; Wawrosch, C.; Uhrin, P.; Temml, V.; Wang, L.; Schwaiger, S.; Heiss, E.H. Discovery and resupply of pharmacologically active plant-derived natural products: A review. Biotechnol. Adv. 2015, 33, 1582-1614. [CrossRef]

12. Ughachukwu, P.; Unekwe, P. Efflux Pump. Mediated Resistance in Chemotherapy. Ann. Med. Health Sci. Res. 2012, 2, 191-198. [CrossRef] [PubMed]

13. Rang, H.P.; Dale, M.M. Rang and Dale's Pharmacology; Elsevier: Rio de Janeiro, Brasil, 2007.

14. Alfarouk, K.O.; Stock, C.-M.; Taylor, S.; Walsh, M.; Muddathir, A.K.; Verduzco, D.; Bashir, A.H.; Mohammed, O.Y.; Elhassan, G.O.; Harguindey, S.; et al. Resistance to cancer chemotherapy: Failure in drug response from ADME to P-gp. Cancer Cell Int. 2015, 15, 1-13. [CrossRef] [PubMed]

15. Vadlapatla, R.K.; Vadlapudi, A.D.; Pal, D.; Mitra, A.K. Mechanisms of drug resistance in cancer chemotherapy: Coordinated role and regulation of efflux transporters and metabolizing enzymes. Curr. Pharm. Des. 2013, 19, 7126-7140. [CrossRef]

16. Wu, Q.; Yang, Z.; Nie, Y.; Shi, Y.; Fan, D. Multi-drug resistance in cancer chemotherapeutics: Mechanisms and lab approaches. Cancer Lett. 2014, 347, 159-166. [CrossRef] [PubMed]

17. Chen, Z.; Shi, T.; Zhang, L.; Zhu, P.; Deng, M.; Huang, C.; Hu, T.; Jiang, L.; Li, J. Mammalian drug efflux transporters of the ATP binding cassette (ABC) family in multidrug resistance: A review of the past decade. Cancer Lett. 2016, 370, 153-164. [CrossRef]

18. Zhang, J.-T. Use of arrays to investigate the contribution of ATP-binding cassette transporters to drug resistance in cancer chemotherapy and prediction of chemosensitivity. Cell Res. 2007, 17, 311-323. [CrossRef]

19. Fojo, A.T.; Ueda, K.; Slamon, D.J.; Poplack, D.; Gottesman, M.; Pastan, I. Expression of a multidrug-resistance gene in human tumors and tissues. Proc. Natl. Acad. Sci. USA 1987, 84, 265-269. [CrossRef]

20. Robinson, K.; Tiriveedhi, V. Perplexing role of P-glycoprotein in tumor microenvironment. Front. Oncol. 2020, 10, 265. [CrossRef]

21. Amawi, H.; Sim, H.-M.; Tiwari, A.K.; Ambudkar, S.V.; Shukla, S. ABC Transporter-Mediated Multidrug-Resistant Cancer. In Drug Transporters in Drug Disposition, Effects and Toxicity; Springer: Singapore, 2019; pp. 549-580.

22. Nobili, S.; Mini, E.; Riganti, C. Multidrug Resistance in Cancer: Pharmacological Strategies from Basic Research to Clinical Issues; Frontiers Media S.A.: Lausanne, Switzerland, 2015.

23. Munoz, M.; Henderson, M.; Haber, M.; Norris, M. Role of the MRP1/ABCC1 multidrug transporter protein in cancer. IUBMB Life 2007, 59, 752-757. [CrossRef]

24. Cho, S.; Lu, M.; He, X.; Ee, P.-L.R.; Bhat, U.; Schneider, E.; Miele, L.; Beck, W.T. Notch1 regulates the expression of the multidrug resistance gene ABCC1/MRP1 in cultured cancer cells. Proc. Natl. Acad. Sci. USA 2011, 108, 20778-20783. [CrossRef]

25. Rosenberg, M.F.; Mao, Q.; Holzenburg, A.; Ford, R.C.; Deeley, R.G.; Cole, S.P. The structure of the multidrug resistance protein 1 (MRP1/ABCC1): Crystallization and single-particle analysis. J. Biol. Chem. 2001, 276, 16076-16082. [CrossRef]

26. Sosnik, A.; Bendayan, R. Drug Efflux Pumps in Cancer Resistance Pathways: From Molecular Recognition and Characterization to Possible Inhibition Strategies in Chemotherapy; Academic Press: San Diego, CA, USA, 2019.

27. Müller, M.; Meijer, C.; Zaman, G.; Borst, P.; Scheper, R.J.; Mulder, N.H.; De Vries, E.; Jansen, P.L. Overexpression of the gene encoding the multidrug resistance-associated protein results in increased ATP-dependent glutathione S-conjugate transport. Proc. Natl. Acad. Sci. USA 1994, 91, 13033-13037. [CrossRef] [PubMed] 
28. Mo, W.; Liu, J.-Y.; Zhang, J.-T. Biochemistry and pharmacology of human ABCC1/MRP1 and its role in detoxification and in multidrug resistance of cancer chemotherapy. In Recent Advances in Cancer Research and Therapy; Elsevier: Amsterdam, The Netherlands, 2012; pp. 371-404.

29. Mo, W.; Zhang, J.-T. Human ABCG2: Structure, function, and its role in multidrug resistance. Int. J. Biochem. Mol. Boil. 2011, 3, $1-27$.

30. Mao, Q.; Unadkat, J.D. Role of the Breast Cancer Resistance Protein (BCRP/ABCG2) in Drug Transport-An Update. AAPS J. 2015, 17, 65-82. [CrossRef] [PubMed]

31. Ma, L.; Liu, T.; Jin, Y.; Wei, J.; Yang, Y.; Zhang, H. ABCG2 is required for self-renewal and chemoresistance of CD133-positive human colorectal cancer cells. Tumor Biol. 2016, 37, 12889-12896. [CrossRef] [PubMed]

32. Horsey, A.J.; Cox, M.H.; Sarwat, S.; Kerr, I.D. The multidrug transporter ABCG2: Still more questions than answers. Biochem. Soc. Trans. 2016, 44, 824-830. [CrossRef] [PubMed]

33. Pan, S.-T.; Li, Z.-L.; He, Z.-X.; Qiu, J.-X.; Zhou, S.-F. Molecular mechanisms for tumour resistance to chemotherapy. Clin. Exp. Pharmacol. Physiol. 2016, 43, 723-737. [CrossRef] [PubMed]

34. Folmer, Y.; Schneider, M.; Blum, H.E.; Hafkemeyer, P. Reversal of drug resistance of hepatocellular carcinoma cells by adenoviral delivery of anti-ABCC2 antisense constructs. Cancer Gene Ther. 2007, 14, 875-884. [CrossRef]

35. Balaji, S.A.; Udupa, N.; Chamallamudi, M.R.; Gupta, V.; Rangarajan, A. Role of the Drug Transporter ABCC3 in Breast Cancer Chemoresistance. PLoS ONE 2016, 11, e0155013. [CrossRef]

36. Chen, Y.; Zhou, H.; Yang, S.; Su, D. Increased ABCC2 expression predicts cisplatin resistance in non-small cell lung cancer. Cell Biochem. Funct. 2021, 39, 277-286. [CrossRef]

37. Guengerich, F.P. Cytochrome P450 and Chemical Toxicology. Chem. Res. Toxicol. 2008, 21, 70-83. [CrossRef]

38. Guengerich, F.P. Mechanisms of cytochrome P450 substrate oxidation: MiniReview. J. Biochem. Mol. Toxicol. 2007, 21, 163-168. [CrossRef] [PubMed]

39. Jančová, P.; Šiller, M. Phase II drug metabolism. Top. Drug Metab. 2012, 2012, 35-60.

40. Cummings, J.; Boyd, G.; Ethell, B.T.; Macpherson, J.S.; Burchell, B.; Smyth, J.F.; Jodrell, D.I. Enhanced clearance of topoisomerase I inhibitors from human colon cancer cells by glucuronidation. Biochem. Pharmacol. 2002, 63, 607-613. [CrossRef]

41. Meijerman, I.; Beijnen, J.H.; Schellens, J.H. Combined action and regulation of phase II enzymes and multidrug resistance proteins in multidrug resistance in cancer. Cancer Treat. Rev. 2008, 34, 505-520. [CrossRef] [PubMed]

42. Joncourt, F.; Buser, K.; Altermatt, H.; Bacchi, M.; Oberli, A.; Cerny, T. Multiple Drug Resistance Parameter Expression in Ovarian Cancer. Gynecol. Oncol. 1998, 70, 176-182. [CrossRef]

43. Patel, N.; Chatterjee, S.K.; Vrbanac, V.; Chung, I.; Mu, C.J.; Olsen, R.R.; Waghorne, C.; Zetter, B.R. Rescue of paclitaxel sensitivity by repression of Prohibitin1 in drug-resistant cancer cells. Proc. Natl. Acad. Sci. USA 2010, 107, 2503-2508. [CrossRef] [PubMed]

44. Green, J.; Robertson, L.; Clark, A.H. Glutathione S-transferase expression in benign and malignant ovarian tumours. Br. J. Cancer 1993, 68, 235-239. [CrossRef]

45. Jardim, B.V.; Moschetta, M.G.; Leonel, C.; Gelaleti, G.B.; Regiani, V.R.; Ferreira, L.C.; Lopes, J.R.; de Campos Zuccari, D.A. Glutathione and glutathione peroxidase expression in breast cancer: An immunohistochemical and molecular study. Oncol. Rep. 2013, 30, 1119-1128. [CrossRef]

46. Yu, P.; Du, Y.; Cheng, X.; Yu, Q.; Huang, L.; Dong, R. Expression of multidrug resistance-associated proteins and their relation to postoperative individualized chemotherapy in gastric cancer. World J. Surg. Oncol. 2014, 12, 307. [CrossRef]

47. Ge, J.; Tian, A.-X.; Wang, Q.-S.; Kong, P.-Z.; Yu, Y.; Li, X.-Q.; Cao, X.-C.; Feng, Y.-M. The GSTP1 105Val Allele Increases Breast Cancer Risk and Aggressiveness but Enhances Response to Cyclophosphamide Chemotherapy in North China. PLoS ONE 2013, 8, e67589. [CrossRef]

48. Wang, H.; Gao, X.; Zhang, X.; Gong, W.; Peng, Z.; Wang, B.; Wang, L.; Chang, S.; Ma, P.; Wang, S. Glutathione S-Transferase Gene Polymorphisms are Associated with an Improved Treatment Response to Cisplatin-Based Chemotherapy in Patients with Non-Small Cell Lung Cancer (NSCLC): A Meta-Analysis. Med. Sci. Monit. 2018, 24, 7482-7492. [CrossRef]

49. Pacholak, L.M.; Amarante, M.K.; Guembarovski, R.L.; Watanabe, M.A.E.; Panis, C. Polymorphisms in GSTT1 and GSTM1 genes as possible risk factors for susceptibility to breast cancer development and their influence in chemotherapy response: A systematic review. Mol. Biol. Rep. 2020, 47, 5495-5501. [CrossRef]

50. Pfeffer, C.M.; Singh, A.T.K. Apoptosis: A Target for Anticancer Therapy. Int. J. Mol. Sci. 2018, 19, 448. [CrossRef]

51. Tummers, B.; Green, D.R. Caspase-8: Regulating life and death. Immunol. Rev. 2017, 277, 76-89. [CrossRef]

52. Kim, R.; Tanabe, K.; Uchida, Y.; Emi, M.; Inoue, H.; Toge, T. Current status of the molecular mechanisms of anticancer druginduced apoptosis. Cancer Chemother. Pharmacol. 2002, 50, 343-352. [CrossRef]

53. Bai, L.; Wang, S. Targeting Apoptosis Pathways for New Cancer Therapeutics. Annu. Rev. Med. 2014, 65, 139-155. [CrossRef]

54. Hassan, M.; Watari, H.; AbuAlmaaty, A.; Ohba, Y.; Sakuragi, N. Apoptosis and Molecular Targeting Therapy in Cancer. BioMed Res. Int. 2014, 2014, 150845. [CrossRef]

55. Warren, C.F.A.; Wong-Brown, M.W.; Bowden, N.A. BCL-2 family isoforms in apoptosis and cancer. Cell Death Dis. 2019, 10, 1-12. [CrossRef]

56. Mortenson, M.; Schlieman, M.; Virudalchalam, S.; Bold, R.J. Overexpression of BCL-2 results in activation of the AKT/NF-kB Cell survival pathway. J. Surg. Res. 2003, 114, 302. [CrossRef] 
57. Buchholz, T.A.; Davis, D.W.; McConkey, D.J.; Symmans, W.F.; Valero, V.; Jhingran, A.; Tucker, S.L.; Pusztai, L.; Cristofanilli, M.; Esteva, F; ; et al. Chemotherapy-Induced Apoptosis and Bcl-2 Levels Correlate with Breast Cancer Response to Chemotherapy. Cancer J. 2003, 9, 33-41. [CrossRef] [PubMed]

58. Sjöström, J.; Blomqvist, C.; von Boguslawski, K.; Bengtsson, N.-O.; Mjaaland, I.; Malmström, P.; Ostenstadt, B.; Wist, E.; Valvere, V.; Takayama, S. The predictive value of bcl-2, bax, bcl-xL, bag-1, fas, and fasL for chemotherapy response in advanced breast cancer. Clin. Cancer Res. 2002, 8, 811-816.

59. Deng, X.; Kornblau, S.M.; Ruvolo, P.P.; May, W.S., Jr. Regulation of Bcl2 phosphorylation and potential significance for leukemic cell chemoresistance. J. Natl. Cancer Inst. Monogr. 2000, 2000, 30-37. [CrossRef]

60. Niero, E.L.; Rocha-Sales, B.; Lauand, C.; Cortez, B.A.; De Souza, M.M.; Rezende-Teixeira, P.; Urabayashi, M.S.; Martens, A.A.; Neves, J.H.; Machado-Santelli, G.M. The multiple facets of drug resistance: One history, different approaches. J. Exp. Clin. Cancer Res. 2014, 33, 37. [CrossRef] [PubMed]

61. Bukowski, K.; Kciuk, M.; Kontek, R. Mechanisms of Multidrug Resistance in Cancer Chemotherapy. Int. J. Mol. Sci. 2020, 21, 3233. [CrossRef]

62. Helleday, T.; Petermann, E.; Lundin, C.; Hodgson, B.; Sharma, R.A. DNA repair pathways as targets for cancer therapy. Nat. Rev. Cancer 2008, 8, 193-204. [CrossRef]

63. Chatterjee, N.; Walker, G.C. Mechanisms of DNA damage, repair, and mutagenesis. Environ. Mol. Mutagen. 2017, 58, 235-263. [CrossRef]

64. El Baiomy, M.A.; El Kashef, W.F. ERCC1 expression in metastatic triple negative breast cancer patients treated with platinum-based chemotherapy. Asian Pac. J. Cancer Prev. 2017, 18, 507-513. [CrossRef]

65. Dupont, C.; Armant, D.R.; Brenner, C.A. Epigenetics: Definition, Mechanisms and Clinical Perspective. In Seminars in Reproductive Medicine; Thieme Medical Publishers: New York, NY, USA, 2009; pp. 351-357.

66. Jones, M.; Beuron, F.; Borg, A.; Nans, A.; Earl, C.P.; Briggs, D.C.; Snijders, A.P.; Bowles, M.; Morris, E.P.; Linch, M.; et al. Cryo-EM structures of the XPF-ERCC1 endonuclease reveal how DNA-junction engagement disrupts an auto-inhibited conformation. Nat. Commun. 2020, 11, 1-14. [CrossRef] [PubMed]

67. Youn, C.-K.; Kim, M.-H.; Cho, H.-J.; Kim, H.-B.; Chang, I.-Y.; Chung, M.-H.; You, H.J. Oncogenic H-Ras Up-Regulates Expression of ERCC1 to Protect Cells from Platinum-Based Anticancer Agents. Cancer Res. 2004, 64, 4849-4857. [CrossRef]

68. Rocha, C.R.R.; Silva, M.M.; Quinet, A.; Cabral-Neto, J.B.; Menck, C.F.M. DNA repair pathways and cisplatin resistance: An intimate relationship. Clinics 2018, 73, e478s. [CrossRef]

69. Olaussen, K.A.; Dunant, A.; Fouret, P.; Brambilla, E.; Andre, F.; Haddad, V.; Taranchon, E.; Filipits, M.; Pirker, R.; Popper, H.H.; et al. DNA Repair by ERCC1 in Non-Small-Cell Lung Cancer and Cisplatin-Based Adjuvant Chemotherapy. N. Engl. J. Med. 2006, 355, 983-991. [CrossRef]

70. Yu, W.; Zhang, L.; Wei, Q.; Shao, A. O6-Methylguanine-DNA Methyltransferase (MGMT): Challenges and New Opportunities in Glioma Chemotherapy. Front. Oncol. 2020, 9, 1547. [CrossRef]

71. Berger, S.; Kouzarides, T.; Shiekhattar, R.; Shilatifard, A. An operational definition of epigenetics. Genes Dev. 2009, 23, 781-783. [CrossRef]

72. Jurkowska, R.Z.; Jurkowski, T.P.; Jeltsch, A. Structure and Function of Mammalian DNA Methyltransferases. ChemBioChem 2011, 12, 206-222. [CrossRef]

73. Zeller, C.; Dai, W.; Steele, N.L.; Siddiq, A.; Walley, A.; Wilhelm-Benartzi, C.; Rizzo, S.; Van Der Zee, A.; Plumb, J.A.; Brown, R. Candidate DNA methylation drivers of acquired cisplatin resistance in ovarian cancer identified by methylome and expression profiling. Oncogene 2012, 31, 4567-4576. [CrossRef]

74. Deaton, A.; Bird, A. CpG islands and the regulation of transcription. Genes Dev. 2011, 25, 1010-1022. [CrossRef]

75. Sumarpo, A.; Ito, K.; Saiki, Y.; Ishizawa, K.; Wang, R.; Chen, N.; Sunamura, M.; Horii, A. Genetic and epigenetic aberrations of ABCB1 synergistically boost the acquisition of taxane resistance in esophageal squamous cancer cells. Biochem. Biophys. Res. Commun. 2020, 526, 586-591. [CrossRef]

76. Ohata, Y.; Shimada, S.; Akiyama, Y.; Mogushi, K.; Nakao, K.; Matsumura, S.; Aihara, A.; Mitsunori, Y.; Ban, D.; Ochiai, T.; et al. Acquired Resistance with Epigenetic Alterations Under Long-Term Antiangiogenic Therapy for Hepatocellular Carcinoma. Mol. Cancer Ther. 2017, 16, 1155-1165. [CrossRef]

77. Bhatla, T.; Wang, J.; Morrison, D.J.; Raetz, E.A.; Burke, M.J.; Brown, P.; Carroll, W.L. Epigenetic reprogramming reverses the relapse-specific gene expression signature and restores chemosensitivity in childhood B-lymphoblastic leukemia. Blood 2012, 119, 5201-5210. [CrossRef] [PubMed]

78. Issa, M.E.; Takhsha, F.S.; Chirumamilla, C.S.; Perez-Novo, C.; Vanden Berghe, W.; Cuendet, M. Epigenetic strategies to reverse drug resistance in heterogeneous multiple myeloma. Clin. Epigenet. 2017, 9, 17. [CrossRef] [PubMed]

79. Zhan, Y.; Li, Y.; Guan, B.; Wang, Z.; Peng, D.; Chen, Z.; He, A.; He, S.; Gong, Y.; Li, X.; et al. Long non-coding RNA HNF1AAS1 promotes proliferation and suppresses apoptosis of bladder cancer cells through upregulating Bcl-2. Oncotarget 2017, 8, 76656-76665. [CrossRef]

80. Wei, J.-W.; Huang, K.; Yang, C.; Kang, C.-S. Non-coding RNAs as regulators in epigenetics. Oncol. Rep. 2016, 37, 3-9. [CrossRef]

81. O'Brien, J.; Hayder, H.; Zayed, Y.; Peng, C. Overview of MicroRNA Biogenesis, Mechanisms of Actions, and Circulation. Front. Endocrinol. 2018, 9, 402. [CrossRef] 
82. Ling, H.; Fabbri, M.; Calin, G.A. MicroRNAs and other non-coding RNAs as targets for anticancer drug development. Nat. Rev. Drug Discov. 2013, 12, 847-865. [CrossRef]

83. Arun, G.; Diermeier, S.D.; Spector, D.L. Therapeutic Targeting of Long Non-Coding RNAs in Cancer. Trends Mol. Med. 2018, 24, 257-277. [CrossRef]

84. Liberti, M.V.; Locasale, J.W. The Warburg Effect: How Does it Benefit Cancer Cells? Trends Biochem. Sci. 2016, 41, 211-218. [CrossRef]

85. Schwartz, L.; Supuran, C.T.; Alfarouk, K.O. The Warburg effect and the hallmarks of cancer. Anti-Cancer Agents Med. Chem. 2017, 17, 164-170. [CrossRef]

86. Vander Heiden, M.G.; Cantley, L.C.; Thompson, C.B. Understanding the Warburg Effect: The Metabolic Requirements of Cell Proliferation. Science 2009, 324, 1029-1033. [CrossRef]

87. Zhou, Y.; Tozzi, F.; Chen, J.; Fan, F.; Xia, L.; Wang, J.; Gao, G.; Zhang, A.; Xia, X.; Brasher, H.; et al. Intracellular ATP Levels Are a Pivotal Determinant of Chemoresistance in Colon Cancer Cells. Cancer Res. 2012, 72, 304-314. [CrossRef] [PubMed]

88. Schneider, V.; Krieger, M.L.; Bendas, G.; Jaehde, U.; Kalayda, G.V. Contribution of intracellular ATP to cisplatin resistance of tumor cells. J. Biol. Inorg. Chem. 2012, 18, 165-174. [CrossRef]

89. Wang, X.; Li, Y.; Qian, Y.; Cao, Y.; Shriwas, P.; Zhang, H.; Chen, X. Extracellular ATP, as an energy and phosphorylating molecule, induces different types of drug resistances in cancer cells through ATP internalization and intracellular ATP level increase. Oncotarget 2017, 8, 87860-87877. [CrossRef] [PubMed]

90. Wilhelm, K.; Ganesan, J.; Müller, T.; Dürr, C.; Grimm, M.; Beilhack, A.; Krempl, C.D.; Sorichter, S.; Gerlach, U.V.; Jüttner, E.; et al . Graft-versus-host disease is enhanced by extracellular ATP activating P2X7R. Nat. Med. 2010, 16, 1434-1438. [CrossRef] [PubMed]

91. Qian, Y.; Wang, X.; Li, Y.; Cao, Y.; Chen, X. Extracellular ATP a New Player in Cancer Metabolism: NSCLC Cells Internalize ATP In Vitro and In Vivo Using Multiple Endocytic Mechanisms. Mol. Cancer Res. 2016, 14, 1087-1096. [CrossRef]

92. Du, Z.; Lovly, C.M. Mechanisms of receptor tyrosine kinase activation in cancer. Mol. Cancer 2018, 17, 58. [CrossRef]

93. Yoganathan, S.; Alagaratnam, A.; Acharekar, N.; Kong, J. Ellagic Acid and Schisandrins: Natural Biaryl Polyphenols with Therapeutic Potential to Overcome Multidrug Resistance in Cancer. Cells 2021, 10, 458. [CrossRef]

94. Wang, Z.; Xie, C.; Huang, Y.; Lam, C.W.K.; Chow, M.S.S. Overcoming chemotherapy resistance with herbal medicines: Past, present and future perspectives. Phytochem. Rev. 2013, 13, 323-337. [CrossRef]

95. Guo, Q.; Li, X.; Cui, M.-N.; Sun, J.-L.; Ji, H.-Y.; Ni, B.-B.; Yan, M.-X. CD13-A key player in multi-drug resistance in cancer chemotherapy. Oncol. Res. Featur. Preclin. Clin. Cancer Ther. 2020, 28, 533-540. [CrossRef]

96. Kita, D.H.; Guragossian, N.; Zattoni, I.F.; Moure, V.R.; Rego, F.G.D.M.; Lusvarghi, S.; Moulenat, T.; Belhani, B.; Picheth, G.; Bouacida, S.; et al. Mechanistic basis of breast cancer resistance protein inhibition by new indeno[1,2-b]indoles. Sci. Rep. 2021, 11, 1-16. [CrossRef]

97. To, K.K.; Wu, X.; Yin, C.; Chai, S.; Yao, S.; Kadioglu, O.; Efferth, T.; Ye, Y.; Lin, G. Reversal of multidrug resistance by Marsdenia tenacissima and its main active ingredients polyoxypregnanes. J. Ethnopharmacol. 2017, 203, 110-119. [CrossRef] [PubMed]

98. Rodríguez-Chávez, J.L.; Méndez-Cuesta, C.A.; Ramírez-Apan, T.; Egas, V.; Ávila, J.L.; Neira-González, A.; Hernández, T.; Espinosa-García, F.J.; Delgado, G. Chemo-sensitizing activity of natural cadinanes from Heterotheca inuloides in human uterine sarcoma cells and their in silico interaction with ABC transporters. Bioorg. Chem. 2019, 91, 103091. [CrossRef] [PubMed]

99. Gote, V.; Nookala, A.; Bolla, P.; Pal, D. Drug Resistance in Metastatic Breast Cancer: Tumor Targeted Nanomedicine to the Rescue. Int. J. Mol. Sci. 2021, 22, 4673. [CrossRef]

100. Nabekura, T. Overcoming Multidrug Resistance in Human Cancer Cells by Natural Compounds. Toxins 2010, 2, 1207-1224. [CrossRef] [PubMed]

101. Teng, Y.-N.; Sheu, M.-J.; Hsieh, Y.-W.; Wang, R.-Y.; Chiang, Y.-C.; Hung, C.-C. $\beta$-carotene reverses multidrug resistant cancer cells by selectively modulating human P-glycoprotein function. Phytomedicine 2016, 23, 316-323. [CrossRef] [PubMed]

102. Li, H.; Krstin, S.; Wink, M. Modulation of multidrug resistant in cancer cells by EGCG, tannic acid and curcumin. Phytomedicine 2018, 50, 213-222. [CrossRef] [PubMed]

103. Teng, Y.-N.; Wang, C.C.N.; Liao, W.-C.; Lan, Y.-H.; Hung, C.-C. Caffeic acid attenuates multi-drug resistance in cancer cells by inhibiting efflux function of human P-glycoprotein. Molecules 2020, 25, 247. [CrossRef]

104. Chang, Y.-T.; Wang, C.C.; Wang, J.-Y.; Lee, T.-E.; Cheng, Y.-Y.; Morris-Natschke, S.L.; Lee, K.-H.; Hung, C.-C. Tenulin and isotenulin inhibit P-glycoprotein function and overcome multidrug resistance in cancer cells. Phytomedicine 2019, 53, $252-262$. [CrossRef]

105. Hano, M.; Tomášová, L.; Šereš, M.; Pavlíková, L.; Breier, A.; Sulová, Z. Interplay between P-Glycoprotein Expression and Resistance to Endoplasmic Reticulum Stressors. Molecules 2018, 23, 337. [CrossRef]

106. Umsumarng, S.; Pitchakarn, P.; Yodkeeree, S.; Punfa, W.; Mapoung, S.; Ramli, R.A.; Pyne, S.G.; Limtrakul, P. Modulation of P-glycoprotein by Stemona alkaloids in human multidrug resistance leukemic cells and structural relationships. Phytomedicine 2017, 34, 182-190. [CrossRef]

107. Xu, W.; Xie, S.; Chen, X.; Pan, S.; Qian, H.; Zhu, X. Effects of Quercetin on the Efficacy of Various Chemotherapeutic Drugs in Cervical Cancer Cells. Drug Des. Devel. Ther. 2021, 15, 577-588. [CrossRef]

108. Singh, A.; Patel, S.K.; Kumar, P.; Das, K.C.; Verma, D.; Sharma, R.; Tripathi, T.; Giri, R.; Martins, N.; Garg, N. Quercetin acts as a P-gp modulator via impeding signal transduction from nucleotide-binding domain to transmembrane domain. J. Biomol. Struct. Dyn. 2020. [CrossRef] 
109. Dei, S.; Braconi, L.; Trezza, A.; Menicatti, M.; Contino, M.; Coronnello, M.; Chiaramonte, N.; Manetti, D.; Perrone, M.G.; Romanelli, M.N.; et al. Modulation of the spacer in N,N-bis(alkanol)amine aryl ester heterodimers led to the discovery of a series of highly potent P-glycoprotein-based multidrug resistance (MDR) modulators. Eur. J. Med. Chem. 2019, 172, 71-94. [CrossRef] [PubMed]

110. Peng, S.; Wang, J.; Lu, C.; Xu, Z.; Chai, J.-J.; Ke, Q.; Deng, X.-Z. Emodin enhances cisplatin sensitivity in non-small cell lung cancer through Pgp downregulation. Oncol. Lett. 2021, 21, 230. [CrossRef]

111. Teng, X.; Wang, S.Y.; Shi, Y.Q.; Fan, X.F.; Liu, S.; Xing, Y.; Guo, Y.Y.; Dong, M. The role of emodin on cisplatin resistance reversal of lung adenocarcinoma A549/DDP cell. Anti-Cancer Drugs 2021, 32, 939-949. [CrossRef] [PubMed]

112. Chang, Y.-T.; Lin, Y.-C.; Sun, L.; Liao, W.-C.; Wang, C.C.N.; Chou, C.-Y.; Morris-Natschke, S.L.; Lee, K.-H.; Hung, C.-C. Wilforine resensitizes multidrug resistant cancer cells via competitive inhibition of P-glycoprotein. Phytomedicine 2020, 71, 153239. [CrossRef] [PubMed]

113. Yang, L.; Li, D.; Tang, P.; Zuo, Y. Curcumin increases the sensitivity of K562/DOX cells to doxorubicin by targeting S100 calcium-binding protein A8 and P-glycoprotein. Oncol. Lett. 2020, 19, 83-92. [CrossRef]

114. Tang, J.; Ji, H.; Ren, J.; Li, M.; Zheng, N.; Wu, L. Solid lipid nanoparticles with TPGS and Brij 78: A co-delivery vehicle of curcumin and piperine for reversing P-glycoprotein-mediated multidrug resistance in vitro. Oncol. Lett. 2017, 13, 389-395. [CrossRef]

115. Khonkarn, R.; Daowtak, K.; Okonogi, S. Chemotherapeutic efficacy enhancement in P-gp-Overexpressing cancer cells by flavonoid-loaded polymeric micelles. AAPS PharmSciTech 2020, 21, 1-12. [CrossRef]

116. Shastrala, K.; Kalam, S.; Damerakonda, K.; Sheshagiri, S.B.B.; Kumar, H.; Guda, R.; Kasula, M.; Bedada, S.K. Synthesis, characterization, and pharmacological evaluation of some metal complexes of quercetin as P-gp inhibitors. Future J. Pharm. Sci. 2021, 7, 1-13.

117. Nair, B.; Anto, R.J.; Sabitha, M.; Nath, L.R. Kaempferol-Mediated Sensitization Enhances Chemotherapeutic Efficacy of Sorafenib Against Hepatocellular Carcinoma: An In Silico and In Vitro Approach. Adv. Pharm. Bull. 2020, 10, 472. [CrossRef]

118. Zhang, Q.; Feng, Y.; Kennedy, D. Multidrug-resistant cancer cells and cancer stem cells hijack cellular systems to circumvent systemic therapies, can natural products reverse this? Cell. Mol. Life Sci. 2016, 74, 777-801. [CrossRef]

119. Hamed, A.R.; Abdel-Azim, N.S.; Shams, K.A.; Hammouda, F.M. Targeting multidrug resistance in cancer by natural chemosensitizers. Bull. Natl. Res. Cent. 2019, 43, 8. [CrossRef]

120. Dallavalle, S.; Dobričić, V.; Lazzarato, L.; Gazzano, E.; Machuqueiro, M.; Pajeva, I.; Tsakovska, I.; Zidar, N.; Fruttero, R. Improvement of conventional anti-cancer drugs as new tools against multidrug resistant tumors. Drug Resist. Updates 2020, 50, 100682. [CrossRef] [PubMed]

121. Syed, S.B.; Arya, H.; Fu, I.-H.; Yeh, T.-K.; Periyasamy, L.; Hsieh, H.-P.; Coumar, M.S. Targeting P-glycoprotein: Investigation of piperine analogs for overcoming drug resistance in cancer. Sci. Rep. 2017, 7, 1-18.

122. Turrini, E.; Sestili, P.; Fimognari, C. Overview of the Anticancer Potential of the "King of Spices" Piper nigrum and Its Main Constituent Piperine. Toxins 2020, 12, 747. [CrossRef] [PubMed]

123. Zhang, Z.-L.; Jiang, Q.-C.; Wang, S.-R. Schisandrin A reverses doxorubicin-resistant human breast cancer cell line by the inhibition of P65 and Stat3 phosphorylation. Breast Cancer 2018, 25, 233-242. [CrossRef]

124. Tinoush, B.; Shirdel, I.; Wink, M. Phytochemicals: Potential Lead Molecules for MDR Reversal. Front. Pharmacol. 2020, 11, 11. [CrossRef]

125. Hee Choi, Y.; Yu, A.-M. ABC transporters in multidrug resistance and pharmacokinetics, and strategies for drug development. Curr. Pharm. Des. 2014, 20, 793-807. [CrossRef]

126. Zhang, Y.-K.; Wang, Y.-J.; Gupta, P.; Chen, Z.-S. Multidrug Resistance Proteins (MRPs) and Cancer Therapy. AAPS J. 2015, 17, 802-812. [CrossRef]

127. El-Readi, M.Z.; Eid, S.; Abdelghany, A.A.; Al-Amoudi, H.S.; Efferth, T.; Wink, M. Resveratrol mediated cancer cell apoptosis, and modulation of multidrug resistance proteins and metabolic enzymes. Phytomedicine 2019, 55, 269-281. [CrossRef]

128. Kweon, S.H.; Song, J.H.; Kim, T.S. Resveratrol-mediated reversal of doxorubicin resistance in acute myeloid leukemia cells via downregulation of MRP1 expression. Biochem. Biophys. Res. Commun. 2010, 395, 104-110. [CrossRef]

129. Li, X.-X.; Dong, Y.; Wang, W.; Wang, H.-L.; Chen, Y.-Y.; Shi, G.-Y.; Yi, J.; Wang, J. Emodin as an effective agent in targeting cancer stem-like side population cells of gallbladder carcinoma. Stem Cell. Dev. 2013, 22, 554-566. [CrossRef]

130. Li, X.; Wang, H.; Wang, J.; Chen, Y.; Yin, X.; Shi, G.; Li, H.; Hu, Z.; Liang, X. Emodin enhances cisplatin-induced cytotoxicity in human bladder cancer cells through ROS elevation and MRP1 downregulation. BMC Cancer 2016, 16, 1-10. [CrossRef]

131. Guo, H.; Liu, F.; Yang, S.; Xue, T. Emodin alleviates gemcitabine resistance in pancreatic cancer by inhibiting MDR1/P-glycoprotein and MRPs expression. Oncol. Lett. 2020, 20, 167. [CrossRef]

132. Krisnamurti, D.G.B.; Wanandi, S.I.; Louisa, M. Curcumin increases the sensitivity of breast cancer cells to tamoxifen by inhibiting MRP2 mrna expression of efflux transporter MRP2. Int. J. Appl. Pharm. 2019, 11, 88-90. [CrossRef]

133. Roy, M.; Mukherjee, S. Reversal of resistance towards cisplatin by curcumin in cervical cancer cells. Asian Pac. J. Cancer Prev. 2014, 15, 1403-1410. [CrossRef] [PubMed]

134. Louisa, M.; Wardhani, B.W. Quercetin improves the efficacy of sorafenib in triple negative breast cancer cells through the modulation of drug efflux transporters expressions. Int. J. Appl. Pharm. 2019, 11, 129-134. [CrossRef]

135. Tang, H.; Zeng, L.; Wang, J.; Zhang, X.; Ruan, Q.; Wang, J.; Cui, S.; Yang, D. Reversal of 5-fluorouracil resistance by EGCG is mediate by inactivation of TFAP2A/VEGF signaling pathway and down-regulation of MDR-1 and P-gp expression in gastric cancer. Oncotarget 2017, 8, 82842. [CrossRef] [PubMed] 
136. La, X.; Zhang, L.; Li, Z.; Li, H.; Yang, Y. (-)-Epigallocatechin Gallate (EGCG) enhances the sensitivity of colorectal cancer cells to 5-FU by inhibiting GRP78/NF-kB/miR-155-5p/MDR1 pathway. J. Agric. Food Chem. 2019, 67, 2510-2518. [CrossRef]

137. Kawahara, I.; Nishikawa, S.; Yamamoto, A.; Kono, Y.; Fujita, T. Assessment of contribution of BCRP to intestinal absorption of various drugs using portal-systemic blood concentration difference model in mice. Pharmacol. Res. Perspect. 2019, 8, e00544. [CrossRef] [PubMed]

138. Zhang, Y.; Zhang, X.-Y.; Zhang, G.-N.; Wang, Y.-J.; Xu, H.; Zhang, D.; Shukla, S.; Liu, L.; Yang, D.-H.; Ambudkar, S.V.; et al. Selective reversal of BCRP-mediated MDR by VEGFR-2 inhibitor ZM323881. Biochem. Pharmacol. 2017, 132, 29-37. [CrossRef] [PubMed]

139. Chen, L.; Manautou, J.E.; Rasmussen, T.P.; Zhong, X.B. Development of precision medicine approaches based on inter-individual variability of BCRP/ABCG2. Acta Pharm. Sin. B 2019, 9, 659-674. [CrossRef] [PubMed]

140. Mahmoud, N.; Saeed, M.E.M.; Sugimoto, Y.; Klauck, S.M.; Greten, H.J.; Efferth, T. Cytotoxicity of nimbolide towards multidrugresistant tumor cells and hypersensitivity via cellular metabolic modulation. Oncotarget 2018, 9, 35762-35779. [CrossRef] [PubMed]

141. Michalak, K.; Wesolowska, O. Polyphenols counteract tumor cell chemoresistance conferred by multidrug resistance proteins. Anti-Cancer Agents Med. Chem. 2012, 12, 880-890. [CrossRef] [PubMed]

142. Ding, Y.; He, J.; Huang, J.; Yu, T.; Shi, X.; Zhang, T.; Yan, G.; Chen, S.; Peng, C. Harmine induces anticancer activity in breast cancer cells via targeting TAZ. Int. J. Oncol. 2019, 54, 1995-2004. [CrossRef]

143. Cartee, L.; Kucera, G.L. Protein kinase C modulation and anticancer drug response. Cancer Investig. 2000, 18, 731-739. [CrossRef]

144. Goel, G.; Makkar, H.P.; Francis, G.; Becker, K. Phorbol esters: Structure, biological activity, and toxicity in animals. Int. J. Toxicol. 2007, 26, 279-288. [CrossRef]

145. Roy, M.; Mukherjee, S.; Sarkar, R.; Biswas, J. Curcumin sensitizes chemotherapeutic drugs via modulation of PKC, telomerase, NF-kB and HDAC in breast cancer. Ther. Deliv. 2011, 2, 1275-1293. [CrossRef]

146. Maurya, A.K.; Vinayak, M. Anticarcinogenic action of quercetin by downregulation of phosphatidylinositol 3-kinase (PI3K) and protein kinase C (PKC) via induction of p53 in hepatocellular carcinoma (HepG2) cell line. Mol. Biol. Rep. 2015, 42, 1419-1429. [CrossRef]

147. Tsuji, K.; Wang, Y.-H.; Takanashi, M.; Odajima, T.; Lee, G.A.; Sugimori, H.; Motoji, T. Overexpression of lung resistance-related protein and P-glycoprotein and response to induction chemotherapy in acute myelogenous leukemia. Hematol. Rep. 2012, 4, e18. [CrossRef]

148. Kulsoom, B.; Shamsi, T.S.; Afsar, N.A. Lung resistance-related protein (LRP) predicts favorable therapeutic outcome in Acute Myeloid Leukemia. Sci. Rep. 2019, 9, 378. [CrossRef]

149. Remy, S.; Litaudon, M. Macrocyclic diterpenoids from euphorbiaceae as a source of potent and selective inhibitors of chikungunya virus replication. Molecules 2019, 24, 2336. [CrossRef] [PubMed]

150. Kraft, A.S.; Anderson, W.B. Phorbol esters increase the amount of Ca 2+, phospholipid-dependent protein kinase associated with plasma membrane. Nature 1983, 301, 621-623. [CrossRef] [PubMed]

151. Xu, R.X.; Pawelczyk, T.; Xia, T.-H.; Brown, S.C. NMR structure of a protein kinase C- $\gamma$ phorbol-binding domain and study of protein-Lipid micelle interactions. Biochemistry 1997, 36, 10709-10717. [CrossRef]

152. Tang, X.Y.; Tang, Y.X.; Xu, P.; Zhou, H.Y.; Han, L. Effect of Peimine on ERCC1 mRNA and LRP Expressions of A549/DDP Multidrug Resistance Cell Line. Zhongguo Zhong Xi Yi Jie He Za Zhi (Chin. J. Integr. Tradit. West. Med.) 2015, 35, 1490-1494.

153. Mackay, H.J.; Twelves, C.J. Targeting the protein kinase C family: Are we there yet? Nat. Rev. Cancer 2007, 7, 554-562. [CrossRef] [PubMed]

154. Swannie, H.C.; Kaye, S.B. Protein kinase C inhibitors. Curr. Oncol. Rep. 2002, 4, 37-46. [CrossRef] [PubMed]

155. Newton, A.C. Protein kinase C: Structure, function, and regulation. J. Biol. Chem. 1995, 270, 28495-28498. [CrossRef]

156. Nishizuka, Y. Intracellular signaling by hydrolysis of phospholipids and activation of protein kinase C. Science 1992, 258, 607-614. [CrossRef]

157. McKernan, L.N.; Momjian, D.; Kulkosky, J. Protein kinase C: One pathway towards the eradication of latent HIV-1 reservoirs. Adv. Virol. 2012, 2012, 805347. [CrossRef]

158. Jian, B.; Zhang, H.; Han, C.; Liu, J. Anti-cancer activities of diterpenoids derived from Euphorbia fischeriana Steud. Molecules 2018, 23, 387. [CrossRef]

159. Pljesa-Ercegovac, M.; Savic-Radojevic, A.; Matic, M.; Coric, V.; Djukic, T.; Radic, T.; Simic, T. Glutathione transferases: Potential targets to overcome chemoresistance in solid tumors. Int. J. Mol. Sci. 2018, 19, 3785. [CrossRef]

160. O'Brien, M.L.; Tew, K.D. Glutathione and related enzymes in multidrug resistance. Eur. J. Cancer 1996, 32, 967-978. [CrossRef]

161. Filomenko, R.; Poirson-Bichat, F.; Billerey, C.; Belon, J.-P.; Garrido, C.; Solary, E.; Bettaieb, A. Atypical protein kinase C $\zeta$ as a target for chemosensitization of tumor cells. Cancer Res. 2002, 62, 1815-1821. [PubMed]

162. Gollapudi, S.; Patel, K.; Jain, V.; Gupta, S. Protein kinase C isoforms in multidrug resistant P388/ADR cells: A possible role in daunorubicin transport. Cancer Lett. 1992, 62, 69-75. [CrossRef]

163. Nabha, S.M.; Glaros, S.; Hong, M.; Lykkesfeldt, A.E.; Schiff, R.; Osborne, K.; Reddy, K.B. Upregulation of PKC- $\delta$ contributes to antiestrogen resistance in mammary tumor cells. Oncogene 2005, 24, 3166-3176. [CrossRef] [PubMed]

164. Fine, R.L.; Chambers, T.C.; Sachs, C.W. P-glycoprotein, multidrug resistance and protein kinase C. Stem Cell. 1996, $14,47-55$. [CrossRef] 
165. Gschwendt, M.; Kittstein, W.; Marks, F. Protein kinase C activation by phorbol esters: Do cysteine-rich regions and pseudosubstrate motifs play a role? Trends Biochem. Sci. 1991, 16, 167-169. [CrossRef]

166. Eid, S.Y.; Althubiti, M.A.; Abdallah, M.E.; Wink, M.; El-Readi, M.Z. The carotenoid fucoxanthin can sensitize multidrug resistant cancer cells to doxorubicin via induction of apoptosis, inhibition of multidrug resistance proteins and metabolic enzymes. Phytomedicine 2020, 77, 153280. [CrossRef]

167. Wang, Z.; Liang, S.; Lian, X.; Liu, L.; Zhao, S.; Xuan, Q.; Guo, L.; Liu, H.; Yang, Y.; Dong, T. Identification of proteins responsible for adriamycin resistance in breast cancer cells using proteomics analysis. Sci. Rep. 2015, 5, 1-11. [CrossRef]

168. Isakov, N. Protein Kinase C (PKC) Isoforms in Cancer, Tumor Promotion and Tumor Suppression; Elsevier: Amsterdam, The Netherlands, 2018; pp. 36-52.

169. Russo, M.; Palumbo, R.; Mupo, A.; Tosto, M.; Iacomino, G.; Scognamiglio, A.; Tedesco, I.; Galano, G.; Russo, G.L. Flavonoid quercetin sensitizes a CD95-resistant cell line to apoptosis by activating protein kinase C $\alpha$. Oncogene 2003, 22, 3330-3342. [CrossRef]

170. Wang, H.-B.; Wang, X.-Y.; Liu, L.-P.; Qin, G.-W.; Kang, T.-G. Tigliane diterpenoids from the Euphorbiaceae and Thymelaeaceae families. Chem. Rev. 2015, 115, 2975-3011. [CrossRef]

171. Di Pietro, G.; Magno, L.A.V.; Rios-Santos, F. Glutathione S-transferases: An overview in cancer research. Expert Opin. Drug Metab. Toxicol. 2010, 6, 153-170. [CrossRef]

172. Hayes, J.D.; Flanagan, J.U.; Jowsey, I.R. Glutathione transferases. Annu. Rev. Pharmacol. Toxicol. 2005, 45, 51-88. [CrossRef]

173. Singh, R.R.; Reindl, K.M. Glutathione S-Transferases in Cancer. Antioxidants 2021, 10, 701. [CrossRef] [PubMed]

174. Osborne, M.J.; de Oliveira, L.C.; Volpon, L.; Zahreddine, H.A.; Borden, K.L.B. Overcoming drug resistance through the development of selective inhibitors of UDP-glucuronosyltransferase enzymes. J. Mol. Biol. 2019, 431, 258-272. [CrossRef] [PubMed]

175. Wu, J.; Henderson, C.; Feun, L.; Van Veldhuizen, P.; Gold, P.; Zheng, H.; Ryan, T.; Blaszkowsky, L.S.; Chen, H.; Costa, M. Phase II study of darinaparsin in patients with advanced hepatocellular carcinoma. Investig. New Drugs 2010, 28, 670-676. [CrossRef] [PubMed]

176. Shen, H.; Kauvar, L.; Tew, K.D. Importance of glutathione and associated enzymes in drug response. Oncol. Res. Featur. Preclin. Clin. Cancer Ther. 1997, 9, 295-302.

177. Al Fayi, M.; Alamri, A.; Rajagopalan, P. IOX-101 Reverses Drug Resistance Through Suppression of Akt/mTOR/NF-kB Signaling in Cancer Stem Cell-Like, Sphere-Forming NSCLC Cell. Oncol. Res. 2020, 28, 177. [CrossRef]

178. Du, Y.; Zheng, Y.; Yu, C.X.; Zhong, L.; Li, Y.; Wu, B.; Hu, W.; Zhu, E.W.; Xie, V.W.; Xu, Q.; et al. The Mechanisms of Yu Ping Feng San in Tracking the Cisplatin-Resistance by Regulating ATP-Binding Cassette Transporter and Glutathione S-Transferase in Lung Cancer Cells. Front. Pharmacol. 2021, 12, 678126. [CrossRef]

179. Li, J.; Ye, T.; Liu, Y.; Kong, L.; Sun, Z.; Liu, D.; Wang, J.; Xing, H.R. Transcriptional activation of Gstp1 by MEK/ERK signaling confers chemo-resistance to cisplatin in lung cancer stem cells. Front. Oncol. 2019, 9, 476. [CrossRef]

180. Yang, M.; Li, Y.; Shen, X.; Ruan, Y.; Lu, Y.; Jin, X.; Song, P.; Guo, Y.; Zhang, X.; Qu, H. CLDN6 promotes chemoresistance through GSTP1 in human breast cancer. J. Exp. Clin. Cancer Res. 2017, 36, 1-15. [CrossRef]

181. Cheng, S.-Y.; Chen, N.-F.; Wen, Z.-H.; Yao, Z.-K.; Tsui, K.-H.; Kuo, H.-M.; Chen, W.-F. Glutathione S-Transferase M3 Is Associated with Glycolysis in Intrinsic Temozolomide-Resistant Glioblastoma Multiforme Cells. Int. J. Mol. Sci. 2021, 22, 7080. [CrossRef]

182. Fruehauf, J.P.; Brem, H.; Brem, S.; Sloan, A.; Barger, G.; Huang, W.; Parker, R. In vitro drug response and molecular markers associated with drug resistance in malignant gliomas. Clin. Cancer Res. 2006, 12, 4523-4532. [CrossRef] [PubMed]

183. Geng, M.; Wang, L.; Chen, X.; Cao, R.; Li, P. The association between chemosensitivity and Pgp, GST- $\pi$ and Topo II expression in gastric cancer. Diagn. Pathol. 2013, 8, 1-5. [CrossRef] [PubMed]

184. Liu, C.-Z.; Liu, W.; Zheng, Y.; Su, J.-M.; Li, J.-J.; Yu, L.; He, X.-D.; Chen, S.-S. PTEN and PDCD4 are Bona Fide Targets of microRNA-21 in Human Cholangiocarcinoma. Chin. Med. Sci. J. 2012, 27, 65-72. [PubMed]

185. Keyvani-Ghamsari, S.; Khorsandi, K.; Gul, A. Curcumin effect on cancer cells' multidrug resistance: An update. Phytother. Res. 2020, 34, 2534-2556. [CrossRef]

186. Andjelkovic, T.; Pesic, M.; Bankovic, J.; Tanic, N.; Markovic, I.D.; Ruzdijic, S. Synergistic effects of the purine analog sulfinosine and curcumin on the multidrug resistant human non-small cell lung carcinoma cell line (NCI-H460/R). Cancer Biol. Ther. 2008, 7, 1024-1032. [CrossRef] [PubMed]

187. Depeille, P.; Cuq, P.; Passagne, I.; Evrard, A.; Vian, L. Combined effects of GSTP1 and MRP1 in melanoma drug resistance. Br. J. Cancer 2005, 93, 216-223. [CrossRef] [PubMed]

188. Wang, W.; Sun, Y.-P.; Huang, X.-Z.; He, M.; Chen, Y.-Y.; Shi, G.-Y.; Li, H.; Yi, J.; Wang, J. Emodin enhances sensitivity of gallbladder cancer cells to platinum drugs via glutathion depletion and MRP1 downregulation. Biochem. Pharmacol. 2010, 79, 1134-1140. [CrossRef] [PubMed]

189. Chen, Y.-Y.; Li, J.; Hu, J.-D.; Zheng, J.; Zheng, Z.-H.; Zhu, L.-F.; Chen, X.-J.; Lin, Z.-X. Reversing effects of emodin on multidrug resistance in resistant HL-60/ADR cells. Zhongguo Shi Yan Xue Ye Xue Za Zhi 2013, 21, 1413-1422. [PubMed]

190. Ou, B.-N.; Tang, H.-H.; Zhang, H.-Y.; Liang, G.; Wei, Y. Preliminary study of the mechanism of reversal effect of emodin in KBV200 cells in vitro. Shandong Med. J. 2011, 24, 20.

191. Majidinia, M.; Mirza-Aghazadeh-Attari, M.; Rahimi, M.; Mihanfar, A.; Karimian, A.; Safa, A.; Yousefi, B. Overcoming multidrug resistance in cancer: Recent progress in nanotechnology and new horizons. IUBMB Life 2020, 72, 855-871. [CrossRef] 
192. Alqarni, M.H.; Foudah, A.I.; Muharram, M.M.; Labrou, N.E. The Interaction of the Flavonoid Fisetin with Human Glutathione Transferase A1-1. Metabolites 2021, 11, 190. [CrossRef]

193. Jin, L.; Xu, M.; Luo, X.-H.; Zhu, X.-F. Stephania tetrandra and ginseng-containing Chinese herbal formulation NSENL reverses cisplatin resistance in lung cancer xenografts. Am. J. Chin. Med. 2017, 45, 385-401. [CrossRef]

194. Liu, C.-M.; Kao, C.-L.; Tseng, Y.-T.; Lo, Y.-C.; Chen, C.-Y. Ginger phytochemicals inhibit cell growth and modulate drug resistance factors in docetaxel resistant prostate cancer cell. Molecules 2017, 22, 1477. [CrossRef]

195. Wang, B.; Shen, C.; Li, Y.; Zhang, T.; Huang, H.; Ren, J.; Hu, Z.; Xu, J.; Xu, B. Oridonin overcomes the gemcitabine resistant PANC-1/Gem cells by regulating GST pi and LRP/1 ERK/JNK signalling. OncoTargets Ther. 2019, 12, 5751. [CrossRef]

196. Martin, L.J. Fucoxanthin and its metabolite fucoxanthinol in cancer prevention and treatment. Mar. Drugs 2015, 13, 4784-4798. [CrossRef]

197. Eid, S.Y.; El-Readi, M.Z.; Wink, M. Carotenoids reverse multidrug resistance in cancer cells by interfering with ABC-transporters. Phytomedicine 2012, 19, 977-987. [CrossRef]

198. Wang, F.; Lu, C.-H.; Willner, I. From cascaded catalytic nucleic acids to enzyme-DNA nanostructures: Controlling reactivity, sensing, logic operations, and assembly of complex structures. Chem. Rev. 2014, 114, 2881-2941. [CrossRef] [PubMed]

199. Liu, L.F. DNA topoisomerase poisons as antitumor drugs. Annu. Rev. Biochem. 1989, 58, 351-375. [CrossRef] [PubMed]

200. Ganguly, A.; Das, B.; Roy, A.; Sen, N.; Dasgupta, S.B.; Mukhopadhayay, S.; Majumder, H.K. Betulinic acid, a catalytic inhibitor of topoisomerase I, inhibits reactive oxygen species-mediated apoptotic topoisomerase I-DNA cleavable complex formation in prostate cancer cells but does not affect the process of cell death. Cancer Res. 2007, 67, 11848-11858. [CrossRef] [PubMed]

201. Wink, M. Molecular modes of action of cytotoxic alkaloids: Trom DNA intercalation, spindle poisoning, topoisomerase inhibition to apoptosis and multiple drug resistance. Alkaloids Chem. Biol. 2007, 64, 1-47.

202. El-Readi, M.Z.; Al-Abd, A.M.; Althubiti, M.A.; Almaimani, R.A.; Al-Amoodi, H.S.; Ashour, M.L.; Wink, M.; Eid, S.Y. Multiple Molecular Mechanisms to Overcome Multidrug Resistance in Cancer by Natural Secondary Metabolites. Front. Pharmacol. 2021, 12, 942. [CrossRef]

203. Li, T.-K.; Liu, L.F. Tumor cell death induced by topoisomerase-targeting drugs. Annu. Rev. Pharmacol. Toxicol. 2001, $41,53-77$. [CrossRef]

204. Austin, C.A.; Sng, J.-H.; Patel, S.; Fisher, L.M. Novel HeLa topoisomerase II is the II $\beta$ isoform: Complete coding sequence and homology with other type II topoisomerases. Biochim. Biophys. Acta 1993, 1172, 283-291. [CrossRef]

205. Chen, W.; Qiu, J.; Shen, Y. Topoisomerase II $\alpha$, rather than II $\beta$, is a promising target in development of anti-cancer drugs. Drug Discov. Ther. 2012, 6, 230-237. [CrossRef]

206. Woessner, R.D.; Mattern, M.R.; Mirabelli, C.K.; Johnson, R.K.; Drake, F.H. Proliferation-and cell cycle-dependent differences in expression of the 170 kilodalton and 180 kilodalton forms of topoisomerase II in NIH-3T3 cells. Cell Growth Differ. 1991, 2, 209-214.

207. Tu, Y.; Wu, Z.; Tan, B.; Yang, A.; Fang, Z. Emodin: Its role in prostate cancer-associated inflammation. Oncol. Rep. 2019, 42, 1259-1271. [CrossRef]

208. Mitani, T.; Ito, Y.; Harada, N.; Nakano, Y.; Inui, H.; Ashida, H.; Yamaji, R. Resveratrol Reduces the Hypoxia-Induced Resistance to Doxorubicin in Breast Cancer Cells. J. Nutr. Sci. Vitaminol. 2014, 60, 122-128. [CrossRef]

209. Zhang, C.; Deng, J.; Liu, D.; Tuo, X.; Yu, Y.; Yang, H.; Wang, N. Nuciferine inhibits proinflammatory cytokines via the PPARs in LPS-induced RAW264. 7 cells. Molecules 2018, 23, 2723. [CrossRef]

210. Ma, Z.; Hano, Y.; Nomura, T.; Chen, Y. Novel quinazoline-quinoline alkaloids with cytotoxic and DNA topoisomerase II inhibitory activities. Bioorg. Med. Chem. Lett. 2004, 14, 1193-1196. [CrossRef]

211. Lin, J.-P.; Lu, H.-F.; Lee, J.-H.; Lin, J.-G.; Hsia, T.-C.; Wu, L.-T.; Chung, J.-G. (-)-Menthol Inhibits DNA Topoisomerases I, II $\alpha$ and $\beta$ and Promotes NF-Î B expression in Human Gastric Cancer SNU-5 Cells. Anticancer Res. 2005, 25, $2069-2074$.

212. Makhey, D.; Gatto, B.; Yu, C.; Liu, A.; Liu, L.F.; LaVoie, E.J. Coralyne and related compounds as mammalian topoisomerase I and topoisomerase II poisons. Bioorg. Med. Chem. 1996, 4, 781-791. [CrossRef]

213. Wang, M.; Liu, Z.-F.; Tang, H.; Chen, B.-A. Application of alkaloids in reversing multidrug resistance in human cancers. Chin. J. Nat. Med. 2018, 16, 561-571.

214. Xue, X.; Qu, X.-J.; Gao, Z.-H.; Sun, C.-C.; Liu, H.-P.; Zhao, C.-R.; Cheng, Y.-N.; Lou, H.-X. Riccardin D, a novel macrocyclic bisbibenzyl, induces apoptosis of human leukemia cells by targeting DNA topoisomerase II. Investig. New Drugs 2012, 30, 212-222. [CrossRef]

215. Greco, O.; Marples, B.; Joiner, M.C.; Scott, S.D. How to overcome (and exploit) tumor hypoxia for targeted gene therapy. J. Cell. Physiol. 2003, 197, 312-325. [CrossRef] [PubMed]

216. Brown, J.M. Exploiting the hypoxic cancer cell: Mechanisms and therapeutic strategies. Mol. Med. Today 2000, 6, 157-162. [CrossRef]

217. Liu, L.; Ning, X.; Sun, L.; Zhang, H.; Shi, Y.; Guo, C.; Han, S.; Liu, J.; Sun, S.; Han, Z. Hypoxia-inducible factor-1 $\alpha$ contributes to hypoxia-induced chemoresistance in gastric cancer. Cancer Sci. 2008, 99, 121-128. [CrossRef] [PubMed]

218. Semenza, G.L. HIF-1: Mediator of physiological and pathophysiological responses to hypoxia. J. Appl. Physiol. 2000, 88, 1474-1480. [CrossRef] [PubMed]

219. Huang, L.E.; Gu, J.; Schau, M.; Bunn, H.F. Regulation of hypoxia-inducible factor $1 \alpha$ is mediated by an $\mathrm{O}_{2}$-dependent degradation domain via the ubiquitin-proteasome pathway. Proc. Natl. Acad. Sci. USA 1998, 95, 7987-7992. [CrossRef] [PubMed] 
220. Xu, S.; Yu, C.; Ma, X.; Li, Y.; Shen, Y.; Chen, Y.; Huang, S.; Zhang, T.; Deng, W.; Wang, Y. IL-6 promotes nuclear translocation of HIF-1 $\alpha$ to aggravate chemoresistance of ovarian cancer cells. Eur. J. Pharmacol. 2021, 894, 173817. [CrossRef] [PubMed]

221. Jin, X.; Gong, L.; Peng, Y.; Li, L.; Liu, G. Enhancer-bound Nrf2 licenses HIF-1 $\alpha$ transcription under hypoxia to promote cisplatin resistance in hepatocellular carcinoma cells. Aging 2021, 13, 364. [CrossRef] [PubMed]

222. Wang, P.; Yan, Q.; Liao, B.; Zhao, L.; Xiong, S.; Wang, J.; Zou, D.; Pan, J.; Wu, L.; Deng, Y. The HIF1 $\alpha /$ HIF2 $\alpha-m i R 210-3 p$ network regulates glioblastoma cell proliferation, dedifferentiation and chemoresistance through EGF under hypoxic conditions. Cell Death Dis. 2020, 11, 1-13. [CrossRef]

223. Sun, L.-T.; Zhang, L.-Y.; Shan, F.-Y.; Shen, M.-H.; Ruan, S.-M. Jiedu Sangen decoction inhibits chemoresistance to 5-fluorouracil of colorectal cancer cells by suppressing glycolysis via PI3K/AKT/HIF-1 $\alpha$ signaling pathway. Chin. J. Nat. Med. 2021, 19, 143-152.

224. Stoeltzing, O.; McCarty, M.F.; Wey, J.S.; Fan, F.; Liu, W.; Belcheva, A.; Bucana, C.D.; Semenza, G.L.; Ellis, L.M. Role of hypoxiainducible factor $1 \alpha$ in gastric cancer cell growth, angiogenesis, and vessel maturation. J. Natl. Cancer Inst. 2004, 96, 946-956. [CrossRef]

225. McMahon, S.; Grondin, F.; McDonald, P.P.; Richard, D.E.; Dubois, C.M. Hypoxia-enhanced expression of the proprotein convertase furin is mediated by hypoxia-inducible factor-1: Impact on the bioactivation of proproteins. J. Biol. Chem. 2005, 280, 6561-6569. [CrossRef]

226. Wang, G.; Xie, G.; Han, L.; Wang, D.; Du, F.; Kong, X.; Su, G. Involvement of hypoxia-inducible factor-1 alpha in the upregulation of P-glycoprotein in refractory epilepsy. Neuroreport 2019, 30, 1191-1196. [CrossRef]

227. Lv, Y.; Zhao, S.; Han, J.; Zheng, L.; Yang, Z.; Zhao, L. Hypoxia-inducible factor-1 $\alpha$ induces multidrug resistance protein in colon cancer. OncoTargets Ther. 2015, 8, 1941. [CrossRef]

228. Wen, Y.; Zhao, R.-Q.; Zhang, Y.-K.; Gupta, P.; Fu, L.-X.; Tang, A.-Z.; Liu, B.-M.; Chen, Z.-S.; Yang, D.-H.; Liang, G. Effect of Y6, an epigallocatechin gallate derivative, on reversing doxorubicin drug resistance in human hepatocellular carcinoma cells. Oncotarget 2017, 8, 29760. [CrossRef]

229. Kathawala, R.J.; Gupta, P.; Ashby Jr, C.R.; Chen, Z.-S. The modulation of ABC transporter-mediated multidrug resistance in cancer: A review of the past decade. Drug Resist. Updates 2015, 18, 1-17. [CrossRef]

230. Li, K.; Li, M.; Luo, Z.; Mao, Y.; Yu, Y.; He, Y.; Zhou, J.; Fei, Y.; Pei, Y.; Cai, K. Overcoming the hypoxia-induced drug resistance in liver tumor by the concurrent use of apigenin and paclitaxel. Biochem. Biophys. Res. Commun. 2020, 526, 321-327. [CrossRef] [PubMed]

231. Huang, X.-Z.; Wang, J.; Huang, C.; Chen, Y.-Y.; Shi, G.-Y.; Hu, Q.-S.; Yi, J. Emodin enhances cytotoxicity of chemotherapeutic drugs in prostate cancer cells: The mechanisms involve ROS-mediated suppression of multidrug resistance and hypoxia inducible factor-1. Cancer Biol. Ther. 2008, 7, 468-475. [CrossRef] [PubMed]

232. Hassan, S.; Peluso, J.; Chalhoub, S.; Idoux Gillet, Y.; Benkirane-Jessel, N.; Rochel, N.; Fuhrmann, G.; Ubeaud-Sequier, G. Quercetin potentializes the respective cytotoxic activity of gemcitabine or doxorubicin on 3D culture of AsPC-1 or HepG2 cells, through the inhibition of HIF-1 $\alpha$ and MDR1. PLoS ONE 2020, 15, e0240676. [CrossRef] [PubMed]

233. Li, Z.; Chen, Y.; An, T.; Liu, P.; Zhu, J.; Yang, H.; Zhang, W.; Dong, T.; Jiang, J.; Zhang, Y. Nuciferine inhibits the progression of glioblastoma by suppressing the SOX2-AKT/STAT3-Slug signaling pathway. J. Exp. Clin. Cancer Res. 2019, 38, 1-15. [CrossRef] [PubMed]

234. Shu, G.; Qiu, Y.; Hao, J.; Fu, Q.; Deng, X. Nuciferine alleviates acute alcohol-induced liver injury in mice: Roles of suppressing hepatic oxidative stress and inflammation via modulating miR-144/Nrf2/HO-1 cascade. J. Funct. Foods 2019, 58, 105-113. [CrossRef]

235. Liu, R.-M.; Xu, P.; Chen, Q.; Feng, S.-L.; Xie, Y. A multiple-targets alkaloid nuciferine overcomes paclitaxel-induced drug resistance in vitro and in vivo. Phytomedicine 2020, 79, 153342. [CrossRef]

236. Xu, T.; Guo, P.; He, Y.; Pi, C.; Wang, Y.; Feng, X.; Hou, Y.; Jiang, Q.; Zhao, L.; Wei, Y. Application of curcumin and its derivatives in tumor multidrug resistance. Phytother. Res. 2020, 34, 2438-2458. [CrossRef]

237. Costea, T.; Vlad, O.C.; Miclea, L.-C.; Ganea, C.; Szöllősi, J.; Mocanu, M.-M. Alleviation of multidrug resistance by flavonoid and non-flavonoid compounds in breast, lung, colorectal and prostate cancer. Int. J. Mol. Sci. 2020, 21, 401. [CrossRef]

238. Yoshida, N.; Takada, T.; Yamamura, Y.; Adachi, I.; Suzuki, H.; Kawakami, J. Inhibitory effects of terpenoids on multidrug resistance-associated protein 2- and breast cancer resistance protein-mediated transport. Drug Metab. Dispos. 2008, 36, 1206-1211. [CrossRef] [PubMed]

239. Zhou, X.; Wang, A.; Wang, L.; Yin, J.; Wang, L.; Di, L.; Hoi, M.P.-M.; Shan, L.; Wu, X.; Wang, Y. A Danshensu-Tetramethylpyrazine Conjugate DT-010 Overcomes Multidrug Resistance in Human Breast Cancer. Front. Pharmacol. 2019, 10, 722. [CrossRef] [PubMed]

240. Chen, C.; Wei, Y.-H. Kanglaite enhances the efficacy of cisplatin in suppression of hepatocellular carcinoma via inhibiting CKLF1 mediated NF-kB pathway and regulating transporter mediated drug efflux. J. Ethnopharmacol. 2021, 264, 113388. [CrossRef] [PubMed]

241. Oh, W.K.; Bin Cho, K.; Hien, T.T.; Kim, T.H.; Kim, H.S.; Dao, T.T.; Han, H.-K.; Kwon, S.-M.; Ahn, S.-G.; Yoon, J.-H.; et al. Amurensin G, a Potent Natural SIRT1 Inhibitor, Rescues Doxorubicin Responsiveness via Down-Regulation of Multidrug Resistance 1. Mol. Pharmacol. 2010, 78, 855-864. [CrossRef] [PubMed]

242. Wang, P.; Yang, H.L.; Yang, Y.J.; Wang, L.; Lee, S.C. Overcome cancer cell drug resistance using natural products. Evid.-Based Complementary Altern. Med. 2015, 2015, 767136. [CrossRef] [PubMed] 
243. Chen, H.J.; Chung, Y.L.; Li, C.Y.; Chang, Y.T.; Wang, C.C.; Lee, H.Y.; Lin, H.Y.; Hung, C.C. Taxifolin resensitizes multidrug resistance cancer cells via uncompetitive inhibition of P-glycoprotein function. Molecules 2018, 23, 3055. [CrossRef]

244. Jaramillo, A.C.; Saig, F.A.; Cloos, J.; Jansen, G.; Peters, G.J. How to overcome ATP-binding cassette drug efflux transportermediated drug resistance? Cancer Drug Resist. 2018, 1, 6-29. [CrossRef]

245. Liu, S.; Li, R.; Qian, J.; Sun, J.; Li, G.; Shen, J.; Xie, Y. Combination Therapy of Doxorubicin and Quercetin on MultidrugResistant Breast Cancer and Their Sequential Delivery by Reduction-Sensitive Hyaluronic Acid-Based Conjugate/d- $\alpha$-Tocopheryl Poly(ethylene glycol) 1000 Succinate Mixed Micelles. Mol. Pharm. 2020, 17, 1415-1427. [CrossRef]

246. Hermawan, A.; Putri, H. Current report of natural product development against breast cancer stem cells. Int. J. Biochem. Cell Biol. 2018, 104, 114-132. [CrossRef] [PubMed]

247. Silva, R.; Vilas-Boas, V.; Carmo, H.; Dinis-Oliveira, R.J.; Carvalho, F.; de Lourdes Bastos, M.; Remião, F.J.P. Therapeutics, Modulation of P-glycoprotein efflux pump: Induction and activation as a therapeutic strategy. Pharmacol. Ther. 2015, 149, 1-123. [CrossRef]

248. Lin, S.; Xie, P.; Luo, M.; Li, Q.; Li, L.; Zhang, J.; Zheng, Q.; Chen, H.; Nan, K. Efficiency against multidrug resistance by co-delivery of doxorubicin and curcumin with a legumain-sensitive nanocarrier. Nano Res. 2018, 11, 3619-3635. [CrossRef]

249. Liu, Y.; Guo, L.; Zloh, M.; Zhang, Y.; Huang, J.; Wang, L. Relevance of Breast Cancer Resistance Protein to Pharmacokinetics of Florfenicol in Chickens: A Perspective from In Vivo and In Vitro Studies. Int. J. Mol. Sci. 2018, 19, 3165. [CrossRef]

250. Senthilkumar, R.; Chen, B.-A.; Cai, X.-H.; Fu, R. Anticancer and multidrug-resistance reversing potential of traditional medicinal plants and their bioactive compounds in leukemia cell lines. Chin. J. Nat. Med. 2014, 12, 881-894. [CrossRef]

251. Sagnou, M.; Novikov, F.N.; Ivanova, E.S.; Alexiou, P.; Stroylov, V.S.; Titov, I.Y.; Tatarskiy, V.V.; Vagida, M.S.; Pelecanou, M.; Shtil, A.A. Novel curcumin derivatives as P-glycoprotein inhibitors: Molecular modeling, synthesis and sensitization of multidrug resistant cells to doxorubicin. Eur. J. Med. Chem. 2020, 198, 112331. [CrossRef] [PubMed]

252. Zong, W.-X.; Ditsworth, D.; Bauer, D.E.; Wang, Z.-Q.; Thompson, C.B. Alkylating DNA damage stimulates a regulated form of necrotic cell death. Genes Dev. 2004, 18, 1272-1282. [CrossRef] [PubMed]

253. Xiang, D.-B.; Zhang, K.-Q.; Zeng, Y.-L.; Yan, Q.-Z.; Shi, Z.; Tuo, Q.-H.; Lin, L.-M.; Xia, B.-H.; Wu, P.; Liao, D.-F. Curcumin: From a controversial "panacea" to effective antineoplastic products. Medicine 2020, 99, e18467. [CrossRef]

254. Liao, X.; Bu, Y.; Jia, Q. Traditional Chinese medicine as supportive care for the management of liver cancer: Past, present, and future. Genes Dis. 2020, 7, 370-379. [CrossRef] [PubMed]

255. Li, W.; Liu, J.; Fu, W.; Zheng, X.; Ren, L.; Liu, S.; Wang, J.; Ji, T.; Du, G. 3-O-acetyl-11-keto- $\beta$-boswellic acid exerts anti-tumor effects in glioblastoma by arresting cell cycle at G2/M phase. J. Exp. Clin. Cancer Res. 2018, 37, 132. [CrossRef]

256. Brown, J.M.; Wilson, G. Apoptosis genes and resistance to cancer therapy: What does the experimental and clinical data tell us? Cancer Biol. Ther. 2003, 2, 477-490. [CrossRef]

257. Kebsa, W.; Lahouel, M.; Rouibah, H.; Zihlif, M.; Ahram, M.; Abu-Irmaileh, B.; Mustafa, E.; Al-Ameer, H.J.; Al Shhab, M.; Malek, Z.; et al. Reversing Multidrug Resistance in Chemo-resistant Human Lung Adenocarcinoma (A549/DOX) Cells by Algerian Propolis Through Direct Inhibiting the P-gp Efflux-pump, G0/G1 Cell Cycle Arrest and Apoptosis Induction. Anti-Cancer Agents Med. Chem. 2019, 18, 1330-1337. [CrossRef]

258. Nandy, S.; Dey, A. Bibenzyls and bisbybenzyls of bryophytic origin as promising source of novel therapeutics: Pharmacology, synthesis and structure-activity. DARU J. Pharm. Sci. 2020, 28, 701-734. [CrossRef]

259. Li, C.; Guan, X.; Xue, H.; Wang, P.; Wang, M.; Gai, X. Reversal of P-glycoprotein-mediated multidrug resistance is induced by saikosaponin D in breast cancer MCF-7/adriamycin cells. Pathol. Res. Pract. 2017, 213, 848-853. [CrossRef]

260. Sharifi-Rad, J.; Ozleyen, A.; Boyunegmez Tumer, T.; Oluwaseun Adetunji, C.; El Omari, N.; Balahbib, A.; Taheri, Y.; Bouyahya, A.; Martorell, M.; Martins, N. Natural products and synthetic analogs as a source of antitumor drugs. Biomolecules $2019,9,679$. [CrossRef]

261. Fuchs, Y.; Steller, H. Programmed cell death in animal development and disease. Cell 2011, 147, 742-758. [CrossRef]

262. Festjens, N.; Vanden Berghe, T.; Vandenabeele, P. Necrosis, a well-orchestrated form of cell demise: Signalling cascades, important mediators and concomitant immune response. Biochim. Biophys. Acta 2006, 1757, 1371-1387. [CrossRef]

263. Hardy, K.; Handyside, A.H.; Winston, R.M. The human blastocyst: Cell number, death and allocation during late preimplantation development in vitro. Development 1989, 107, 597-604. [CrossRef]

264. Lockshin, R.A.; Zakeri, Z. Cell death in health and disease. J. Cell. Mol. Med. 2007, 11, 1214-1224. [CrossRef]

265. Golstein, P.; Kroemer, G. Cell death by necrosis: Towards a molecular definition. Trends Biochem. Sci. 2007, 32, 37-43. [CrossRef] [PubMed]

266. Laster, S.M.; Wood, J.G.; Gooding, L.R. Tumor necrosis factor can induce both apoptic and necrotic forms of cell lysis. J. Immunol. 1988, 141, 2629-2634. [PubMed]

267. Degterev, A.; Huang, Z.; Boyce, M.; Li, Y.; Jagtap, P.; Mizushima, N.; Cuny, G.D.; Mitchison, T.J.; Moskowitz, M.A.; Yuan, J. Chemical inhibitor of nonapoptotic cell death with therapeutic potential for ischemic brain injury. Nat. Chem. Biol. 2005, 1, 112-119. [CrossRef] [PubMed]

268. Jin, G.; Liu, Y.; Xu, P.; Jin, G. Induction of Necroptosis in Human Breast Cancer Drug-Resistant Cells by SMAC Analog LCL161 After Caspase Inhibition Requires RIP3. Die Pharmazie 2019, 74, 363-368. [CrossRef]

269. Wang, Y.; Hao, F.; Nan, Y.; Qu, L.; Na, W.; Jia, C.; Chen, X. PKM2 Inhibitor Shikonin Overcomes the Cisplatin Resistance in Bladder Cancer by Inducing Necroptosis. Int. J. Biol. Sci. 2018, 14, 1883-1891. [CrossRef] 
270. Choi, M.E.; Price, D.R.; Ryter, S.W.; Choi, A.M.K. Necroptosis: A crucial pathogenic mediator of human disease. JCI Insight 2019, 4, e128834. [CrossRef]

271. Conrad, M.; Angeli, J.P.; Vandenabeele, P.; Stockwell, B.R. Regulated necrosis: Disease relevance and therapeutic opportunities. Nat. Rev. Drug Discov. 2016, 15, 348-366. [CrossRef] [PubMed]

272. Weinlich, R.; Oberst, A.; Beere, H.M.; Green, D.R. Necroptosis in development, inflammation and disease. Nat. Rev. Mol. Cell Biol. 2017, 18, 127-136. [CrossRef] [PubMed]

273. Bertrand, M.J.; Milutinovic, S.; Dickson, K.M.; Ho, W.C.; Boudreault, A.; Durkin, J.; Gillard, J.W.; Jaquith, J.B.; Morris, S.J.; Barker, P.A. cIAP1 and cIAP2 facilitate cancer cell survival by functioning as E3 ligases that promote RIP1 ubiquitination. Mol. Cell 2008, 30, 689-700. [CrossRef] [PubMed]

274. Hsu, H.; Xiong, J.; Goeddel, D.V. The TNF receptor 1-associated protein TRADD signals cell death and NF-kappa B activation. Cell 1995, 81, 495-504. [CrossRef]

275. Ding, Y.; Li, S.; Ge, W.; Liu, Z.; Zhang, X.; Wang, M.; Chen, T.; Chen, Y.; Zhang, Q. Design and synthesis of parthenolide and 5-fluorouracil conjugates as potential anticancer agents against drug resistant hepatocellular carcinoma. Eur. J. Med. Chem. 2019, 183, 111706. [CrossRef]

276. Gao, L.; Zhao, P.; Li, Y.; Yang, D.; Hu, P.; Li, L.; Cheng, Y.; Yao, H. Reversal of P-glycoprotein-mediated multidrug resistance by novel curcumin analogues in paclitaxel-resistant human breast cancer cells. Biochem. Cell Biol. 2020, 98, 484-491. [CrossRef]

277. Mirzaei, S.A.; Reiisi, S.; Tabari, P.G.; Shekari, A.; Aliakbari, F.; Azadfallah, E.; Elahian, F. Broad blocking of MDR efflux pumps by acetylshikonin and acetoxyisovalerylshikonin to generate hypersensitive phenotype of malignant carcinoma cells. Sci. Rep. 2018, 8, 1-8. [CrossRef]

278. Wong, I.L.K.; Wang, X.-K.; Liu, Z.; Sun, W.; Li, F.-X.; Wang, B.-C.; Li, P.; Wan, S.-B.; Chow, L.M.C. Synthesis and evaluation of stereoisomers of methylated catechin and epigallocatechin derivatives on modulating P-glycoprotein-mediated multidrug resistance in cancers. Eur. J. Med. Chem. 2021, 113795. [CrossRef]

279. Wang, L.; Du, F.; Wang, X. TNF-alpha induces two distinct caspase-8 activation pathways. Cell 2008, 133, 693-703. [CrossRef]

280. He, S.; Liang, Y.; Shao, F.; Wang, X. Toll-like receptors activate programmed necrosis in macrophages through a receptor-interacting kinase-3-mediated pathway. Proc. Natl. Acad. Sci. USA 2011, 108, 20054-20059. [CrossRef]

281. Holler, N.; Zaru, R.; Micheau, O.; Thome, M.; Attinger, A.; Valitutti, S.; Bodmer, J.L.; Schneider, P.; Seed, B.; Tschopp, J. Fas triggers an alternative, caspase-8-independent cell death pathway using the kinase RIP as effector molecule. Nat. Immunol. 2000, 1, 489-495. [CrossRef]

282. Seya, T.; Shime, H.; Takaki, H.; Azuma, M.; Oshiumi, H.; Matsumoto, M. TLR3/TICAM-1 signaling in tumor cell RIP3-dependent necroptosis. OncoImmunology 2012, 1, 917-923. [CrossRef]

283. Chen, D.; Yu, J.; Zhang, L. Necroptosis: An alternative cell death program defending against cancer. Biochim. Biophys. Acta 2016, 1865, 228-236. [CrossRef]

284. Gong, Y.; Fan, Z.; Luo, G.; Yang, C.; Huang, Q.; Fan, K.; Cheng, H.; Jin, K.; Ni, Q.; Yu, X.; et al. The role of necroptosis in cancer biology and therapy. Mol. Cancer 2019, 18, 100. [CrossRef]

285. Philipp, S.; Sosna, J.; Adam, D. Cancer and necroptosis: Friend or foe? Cell. Mol. Life Sci. 2016, 73, 2183-2193. [CrossRef] [PubMed]

286. Vandenabeele, P.; Galluzzi, L.; Vanden Berghe, T.; Kroemer, G. Molecular mechanisms of necroptosis: An ordered cellular explosion. Nat. Rev. Mol. Cell Biol. 2010, 11, 700-714. [CrossRef] [PubMed]

287. Galluzzi, L.; Kepp, O.; Krautwald, S.; Kroemer, G.; Linkermann, A. Molecular mechanisms of regulated necrosis. Semin. Cell Dev. Biol. 2014, 35, 24-32. [CrossRef] [PubMed]

288. Zhou, W.; Yuan, J. Necroptosis in health and diseases. Semin. Cell Dev. Biol. 2014, 35, 14-23. [CrossRef]

289. Galluzzi, L.; Vitale, I.; Aaronson, S.A.; Abrams, J.M.; Adam, D.; Agostinis, P.; Alnemri, E.S.; Altucci, L.; Amelio, I.; Andrews, D.W.; et al. Molecular mechanisms of cell death: Recommendations of the Nomenclature Committee on Cell Death 2018. Cell Death Differ. 2018, 25, 486-541. [CrossRef]

290. Shimada, K.; Skouta, R.; Kaplan, A.; Yang, W.S.; Hayano, M.; Dixon, S.J.; Brown, L.M.; Valenzuela, C.A.; Wolpaw, A.J.; Stockwell, B.R. Global survey of cell death mechanisms reveals metabolic regulation of ferroptosis. Nat. Chem. Biol. 2016, 12, 497-503. [CrossRef]

291. Hu, X.; Xuan, Y. Bypassing cancer drug resistance by activating multiple death pathways-A proposal from the study of circumventing cancer drug resistance by induction of necroptosis. Cancer Lett. 2008, 259, 127-137. [CrossRef] [PubMed]

292. Su, Z.; Yang, Z.; Xu, Y.; Chen, Y.; Yu, Q. Apoptosis, autophagy, necroptosis, and cancer metastasis. Mol. Cancer 2015, 14, 48. [CrossRef] [PubMed]

293. Radogna, F.; Dicato, M.; Diederich, M. Cancer-type-specific crosstalk between autophagy, necroptosis and apoptosis as a pharmacological target. Biochem. Pharmacol. 2015, 94, 1-11. [CrossRef] [PubMed]

294. Long, J.S.; Ryan, K.M. New frontiers in promoting tumour cell death: Targeting apoptosis, necroptosis and autophagy. Oncogene 2012, 31, 5045-5060. [CrossRef] [PubMed]

295. Vanden Berghe, T.; Linkermann, A.; Jouan-Lanhouet, S.; Walczak, H.; Vandenabeele, P. Regulated necrosis: The expanding network of non-apoptotic cell death pathways. Nat. Rev. Mol. Cell Biol. 2014, 15, 135-147. [CrossRef]

296. Han, W.; Xie, J.; Li, L.; Liu, Z.; Hu, X. Necrostatin-1 reverts shikonin-induced necroptosis to apoptosis. Apoptosis 2009, 14, 674-686. [CrossRef] 
297. Kroemer, G.; Piacentini, M. Dying to survive-Apoptosis, necroptosis, autophagy as supreme experiments of nature. Int. J. Dev. Biol. 2015, 59, 5-9. [CrossRef]

298. Dashzeveg, N.; Yoshida, K. Cell death decision by p53 via control of the mitochondrial membrane. Cancer Lett. 2015, 367, 108-112. [CrossRef]

299. Jia, L.T.; Zhang, R.; Shen, L.; Yang, A.G. Regulators of carcinogenesis: Emerging roles beyond their primary functions. Cancer Lett. 2015, 357, 75-82. [CrossRef]

300. Zamaraev, A.V.; Kopeina, G.S.; Zhivotovsky, B.; Lavrik, I.N. Cell death controlling complexes and their potential therapeutic role. Cell. Mol. Life Sci. 2015, 72, 505-517. [CrossRef]

301. Huang, Y.; Wu, J.Z.; Li, J.Y.; Xu, W. Know the enemy as well as the weapons in hand: The aberrant death pathways and therapeutic agents in chronic lymphocytic leukemia. Am. J. Cancer Res. 2015, 5, 2361-2375.

302. Liu, P.; Xu, B.; Shen, W.; Zhu, H.; Wu, W.; Fu, Y.; Chen, H.; Dong, H.; Zhu, Y.; Miao, K.; et al. Dysregulation of TNF $\alpha$-induced necroptotic signaling in chronic lymphocytic leukemia: Suppression of CYLD gene by LEF1. Leukemia 2012, 26, 1293-1300. [CrossRef] [PubMed]

303. Zhang, M.; Harashima, N.; Moritani, T.; Huang, W.; Harada, M. The Roles of ROS and Caspases in TRAIL-Induced Apoptosis and Necroptosis in Human Pancreatic Cancer Cells. PLoS ONE 2015, 10, e0127386. [CrossRef]

304. Schmidt, S.V.; Seibert, S.; Walch-Rückheim, B.; Vicinus, B.; Kamionka, E.M.; Pahne-Zeppenfeld, J.; Solomayer, E.F.; Kim, Y.J.; Bohle, R.M.; Smola, S. RIPK3 expression in cervical cancer cells is required for PolyIC-induced necroptosis, IL-1 $\alpha$ release, and efficient paracrine dendritic cell activation. Oncotarget 2015, 6, 8635-8647. [CrossRef] [PubMed]

305. Melo-Lima, S.; Lopes, M.C.; Mollinedo, F. ERK1/2 acts as a switch between necrotic and apoptotic cell death in ether phospholipid edelfosine-treated glioblastoma cells. Pharmacol. Res. 2015, 95-96, 2-11. [CrossRef] [PubMed]

306. Melo-Lima, S.; Celeste Lopes, M.; Mollinedo, F. Necroptosis is associated with low procaspase- 8 and active RIPK1 and -3 in human glioma cells. Oncoscience 2014, 1, 649-664. [CrossRef] [PubMed]

307. Chen, G.; Cheng, X.; Zhao, M.; Lin, S.; Lu, J.; Kang, J.; Yu, X. RIP1-dependent Bid cleavage mediates TNF $\alpha$-induced but Caspase-3-independent cell death in L929 fibroblastoma cells. Apoptosis 2015, 20, 92-109. [CrossRef] [PubMed]

308. Nugues, A.L.; El Bouazzati, H.; Hétuin, D.; Berthon, C.; Loyens, A.; Bertrand, E.; Jouy, N.; Idziorek, T.; Quesnel, B. RIP3 is downregulated in human myeloid leukemia cells and modulates apoptosis and caspase-mediated p65/RelA cleavage. Cell Death Dis. 2014, 5, e1384. [CrossRef] [PubMed]

309. Nomura, M.; Ueno, A.; Saga, K.; Fukuzawa, M.; Kaneda, Y. Accumulation of cytosolic calcium induces necroptotic cell death in human neuroblastoma. Cancer Res. 2014, 74, 1056-1066. [CrossRef] [PubMed]

310. Fu, Z.; Deng, B.; Liao, Y.; Shan, L.; Yin, F.; Wang, Z.; Zeng, H.; Zuo, D.; Hua, Y.; Cai, Z. The anti-tumor effect of shikonin on osteosarcoma by inducing RIP1 and RIP3 dependent necroptosis. BMC Cancer 2013, 13, 580. [CrossRef]

311. Kroemer, G.; Galluzzi, L.; Vandenabeele, P.; Abrams, J.; Alnemri, E.S.; Baehrecke, E.H.; Blagosklonny, M.V.; El-Deiry, W.S.; Golstein, P.; Green, D.R.; et al. Classification of cell death: Recommendations of the Nomenclature Committee on Cell Death 2009. Cell Death Differ. 2009, 16, 3-11. [CrossRef] [PubMed]

312. Li, J.; Yin, Q.; Wu, H. Structural basis of signal transduction in the TNF receptor superfamily. Adv. Immunol. 2013, 119, 135-153. [CrossRef] [PubMed]

313. Newton, K. RIPK1 and RIPK3: Critical regulators of inflammation and cell death. Trends Cell Biol. 2015, 25, 347-353. [CrossRef]

314. Wallach, D.; Kang, T.B.; Yang, S.H.; Kovalenko, A. The in vivo significance of necroptosis: Lessons from exploration of caspase-8 function. Cytokine Growth Factor Rev. 2014, 25, 157-165. [CrossRef] [PubMed]

315. Christofferson, D.E.; Li, Y.; Yuan, J. Control of life-or-death decisions by RIP1 kinase. Annu. Rev. Physiol. 2014, 76, 129-150. [CrossRef]

316. Thomas, L.R.; Henson, A.; Reed, J.C.; Salsbury, F.R.; Thorburn, A. Direct binding of Fas-associated death domain (FADD) to the tumor necrosis factor-related apoptosis-inducing ligand receptor DR5 is regulated by the death effector domain of FADD. J. Biol. Chem. 2004, 279, 32780-32785. [CrossRef]

317. Kaczmarek, A.; Vandenabeele, P.; Krysko, D. Necroptosis: The Release of Damage-Associated Molecular Patterns and Its Physiological Relevance. Immunity 2013, 38, 209-223. [CrossRef]

318. Greten, F.; Eckmann, L.; Greten, T.F.; Park, J.M.; Li, Z.-W.; Egan, L.J.; Kagnoff, M.F.; Karin, M. IKK $\beta$ Links Inflammation and Tumorigenesis in a Mouse Model of Colitis-Associated Cancer. Cell 2004, 118, 285-296. [CrossRef]

319. Grivennikov, S.I.; Greten, F.R.; Karin, M. Immunity, inflammation, and cancer. Cell 2010, 140, 883-899. [CrossRef] [PubMed]

320. Wang, Q.; Ju, X.; Zhou, Y.; Chen, K. Necroptotic cells release find-me signal and are engulfed without proinflammatory cytokine production. In Vitro Cell. Dev. Biol. Anim. 2015, 51, 1033-1039. [CrossRef] [PubMed]

321. Duffy, M.J.; McGowan, P.M.; Gallagher, W.M. Cancer invasion and metastasis: Changing views. J. Pathol. 2008, $214,283-293$. [CrossRef] [PubMed]

322. Huang, C.; Luo, Y.; Zhao, J.; Yang, F.; Zhao, H.; Fan, W.; Ge, P. Shikonin kills glioma cells through necroptosis mediated by RIP-1. PLoS ONE 2013, 8, e66326. [CrossRef]

323. Kim, Y.S.; Morgan, M.J.; Choksi, S.; Liu, Z.G. TNF-induced activation of the Nox1 NADPH oxidase and its role in the induction of necrotic cell death. Mol. Cell 2007, 26, 675-687. [CrossRef] [PubMed]

324. Steinhart, L.; Belz, K.; Fulda, S. Smac mimetic and demethylating agents synergistically trigger cell death in acute myeloid leukemia cells and overcome apoptosis resistance by inducing necroptosis. Cell Death Dis. 2013, 4, e802. [CrossRef] 
325. Yan, C.; Oh, J.S.; Yoo, S.H.; Lee, J.S.; Yoon, Y.G.; Oh, Y.J.; Jang, M.S.; Lee, S.Y.; Yang, J.; Lee, S.H.; et al. The targeted inhibition of mitochondrial Hsp90 overcomes the apoptosis resistance conferred by Bcl-2 in Hep3B cells via necroptosis. Toxicol. Appl. Pharmacol. 2013, 266, 9-18. [CrossRef]

326. Smith, C.C.; Yellon, D.M. Necroptosis, necrostatins and tissue injury. J. Cell. Mol. Med. 2011, 15, 1797-1806. [CrossRef]

327. Chen, W.; Wu, J.; Li, L.; Zhang, Z.; Ren, J.; Liang, Y.; Chen, F.; Yang, C.; Zhou, Z.; Su, S.S.; et al. Ppm1b negatively regulates necroptosis through dephosphorylating Rip3. Nat. Cell Biol. 2015, 17, 434-444. [CrossRef]

328. Huang, C.Y.; Kuo, W.T.; Huang, Y.C.; Lee, T.C.; Yu, L.C. Resistance to hypoxia-induced necroptosis is conferred by glycolytic pyruvate scavenging of mitochondrial superoxide in colorectal cancer cells. Cell Death Dis. 2013, 4, e622. [CrossRef]

329. Zhang, S.F.; Wang, X.L.; Yang, X.Q.; Chen, N. Autophagy-associated targeting pathways of natural products during cancer treatment. Asian Pac. J. Cancer Prev. 2014, 15, 10557-10563. [CrossRef] [PubMed]

330. Kim, J.; Park, E.J. Cytotoxic anticancer candidates from natural resources. Curr. Med. Chem. Anti-Cancer Agents 2002, 2, 485-537. [CrossRef] [PubMed]

331. Kinghorn, A.D.; Chin, Y.W.; Swanson, S.M. Discovery of natural product anticancer agents from biodiverse organisms. Curr. Opin. Drug Discov. Dev. 2009, 12, 189-196.

332. Meiyanto, E.; Hermawan, A.; Anindyajati, A. Natural products for cancer-targeted therapy: Citrus flavonoids as potent chemopreventive agents. Asian Pac. J. Cancer Prev. 2012, 13, 427-436. [CrossRef] [PubMed]

333. Wang, N.; Feng, Y. Elaborating the role of natural products-induced autophagy in cancer treatment: Achievements and artifacts in the state of the art. BioMed Res. Int. 2015, 2015, 934207. [CrossRef]

334. Gali-Muhtasib, H.; Hmadi, R.; Kareh, M.; Tohme, R.; Darwiche, N. Cell death mechanisms of plant-derived anticancer drugs: Beyond apoptosis. Apoptosis 2015, 20, 1531-1562. [CrossRef]

335. Kravchenko, J.; Corsini, E.; Williams, M.A.; Decker, W.; Manjili, M.H.; Otsuki, T.; Singh, N.; Al-Mulla, F.; Al-Temaimi, R.; Amedei, A.; et al. Chemical compounds from anthropogenic environment and immune evasion mechanisms: Potential interactions. Carcinogenesis 2015, 36 (Suppl. 1), S111-S127. [CrossRef]

336. Luo, X.; Yu, X.; Liu, S.; Deng, Q.; Liu, X.; Peng, S.; Li, H.; Liu, J.; Cao, Y. The role of targeting kinase activity by natural products in cancer chemoprevention and chemotherapy (Review). Oncol. Rep. 2015, 34, 547-554. [CrossRef]

337. Barrera, L.N.; Cassidy, A.; Johnson, I.T.; Bao, Y.; Belshaw, N.J. Epigenetic and antioxidant effects of dietary isothiocyanates and selenium: Potential implications for cancer chemoprevention. Proc. Nutr. Soc. 2012, 71, 237-245. [CrossRef]

338. Shen, M.; Chan, T.H.; Dou, Q.P. Targeting tumor ubiquitin-proteasome pathway with polyphenols for chemosensitization. Anti-Cancer Agents Med. Chem. 2012, 12, 891-901. [CrossRef] [PubMed]

339. Cerella, C.; Dicato, M.; Jacob, C.; Diederich, M. Chemical properties and mechanisms determining the anti-cancer action of garlic-derived organic sulfur compounds. Anti-Cancer Agents Med. Chem. 2011, 11, 267-271. [CrossRef] [PubMed]

340. Huang, J.; Plass, C.; Gerhauser, C. Cancer chemoprevention by targeting the epigenome. Curr. Drug Targets 2011, 12, 1925-1956. [CrossRef]

341. Fulda, S. Modulation of apoptosis by natural products for cancer therapy. Planta Med. 2010, 76, 1075-1079. [CrossRef] [PubMed]

342. Wang, S.; Penchala, S.; Prabhu, S.; Wang, J.; Huang, Y. Molecular basis of traditional Chinese medicine in cancer chemoprevention. Curr. Drug Discov. Technol. 2010, 7, 67-75. [CrossRef]

343. Sarkar, F.H.; Li, Y. Harnessing the fruits of nature for the development of multi-targeted cancer therapeutics. Cancer Treat. Rev. 2009, 35, 597-607. [CrossRef]

344. Xu, B.; Xu, M.; Tian, Y.; Yu, Q.; Zhao, Y.; Chen, X.; Mi, P.; Cao, H.; Zhang, B.; Song, G.; et al. Matrine induces RIP3-dependent necroptosis in cholangiocarcinoma cells. Cell Death Discov. 2017, 3, 16096. [CrossRef]

345. Yu, X.; Deng, Q.; Li, W.; Xiao, L.; Luo, X.; Liu, X.; Yang, L.; Peng, S.; Ding, Z.; Feng, T.; et al. Neoalbaconol induces cell death through necroptosis by regulating RIPK-dependent autocrine TNF $\alpha$ and ROS production. Oncotarget 2015, 6, 1995-2008. [CrossRef]

346. Andújar, I.; Ríos, J.L.; Giner, R.M.; Recio, M.C. Pharmacological properties of shikonin-A review of literature since 2002. Planta Med. 2013, 79, 1685-1697. [CrossRef]

347. Dong, X.; Fu, J.; Yin, X.; Cao, S.; Li, X.; Lin, L.; Ni, J. Emodin: A Review of its Pharmacology, Toxicity and Pharmacokinetics. Phytother. Res. 2016, 30, 1207-1218. [CrossRef]

348. Zhou, J.; Li, G.; Han, G.; Feng, S.; Liu, Y.; Chen, J.; Liu, C.; Zhao, L.; Jin, F. Emodin induced necroptosis in the glioma cell line U251 via the TNF- $\alpha /$ RIP1/RIP3 pathway. Investig. New Drugs 2020, 38, 50-59. [CrossRef]

349. Mbaveng, A.T.; Bitchagno, G.T.M.; Kuete, V.; Tane, P.; Efferth, T. Cytotoxicity of ungeremine towards multi-factorial drug resistant cancer cells and induction of apoptosis, ferroptosis, necroptosis and autophagy. Phytomedicine 2019, 60, 152832. [CrossRef]

350. Piao, J.L.; Cui, Z.G.; Furusawa, Y.; Ahmed, K.; Rehman, M.U.; Tabuchi, Y.; Kadowaki, M.; Kondo, T. The molecular mechanisms and gene expression profiling for shikonin-induced apoptotic and necroptotic cell death in U937 cells. Chem. Biol. Interact. 2013, 205, 119-127. [CrossRef] [PubMed]

351. Deng, B.; Qiu, B. Shikonin inhibits invasiveness of osteosarcoma through MMP13 suppression. Tumour Biol. 2015, 36, 9311-9317. [CrossRef] [PubMed]

352. Zhao, Q.; Kretschmer, N.; Bauer, R.; Efferth, T. Shikonin and its derivatives inhibit the epidermal growth factor receptor signaling and synergistically kill glioblastoma cells in combination with erlotinib. Int. J. Cancer 2015, 137, 1446-1456. [CrossRef] [PubMed] 
353. Wada, N.; Kawano, Y.; Fujiwara, S.; Kikukawa, Y.; Okuno, Y.; Tasaki, M.; Ueda, M.; Ando, Y.; Yoshinaga, K.; Ri, M.; et al. Shikonin, dually functions as a proteasome inhibitor and a necroptosis inducer in multiple myeloma cells. Int. J. Oncol. 2015, 46, 963-972. [CrossRef] [PubMed]

354. Han, W.; Xie, J.; Fang, Y.; Wang, Z.; Pan, H. Nec-1 enhances shikonin-induced apoptosis in leukemia cells by inhibition of RIP-1 and ERK1/2. Int. J. Mol. Sci. 2012, 13, 7212-7225. [CrossRef]

355. Xuan, Y.; Hu, X. Naturally-occurring shikonin analogues-a class of necroptotic inducers that circumvent cancer drug resistance. Cancer Lett. 2009, 274, 233-242. [CrossRef]

356. Hu, X.; Han, W.; Li, L. Targeting the weak point of cancer by induction of necroptosis. Autophagy 2007, 3, 490-492. [CrossRef]

357. Han, W.; Li, L.; Qiu, S.; Lu, Q.; Pan, Q.; Gu, Y.; Luo, J.; Hu, X. Shikonin circumvents cancer drug resistance by induction of a necroptotic death. Mol. Cancer Ther. 2007, 6, 1641-1649. [CrossRef] [PubMed]

358. Jang, S.Y.; Lee, J.K.; Jang, E.H.; Jeong, S.Y.; Kim, J.H. Shikonin blocks migration and invasion of human breast cancer cells through inhibition of matrix metalloproteinase-9 activation. Oncol. Rep. 2014, 31, 2827-2833. [CrossRef]

359. Li, W.; Liu, J.; Jackson, K.; Shi, R.; Zhao, Y. Sensitizing the therapeutic efficacy of taxol with shikonin in human breast cancer cells. PLoS ONE 2014, 9, e94079. [CrossRef] [PubMed]

360. Yao, Y.; Zhou, Q. A novel antiestrogen agent Shikonin inhibits estrogen-dependent gene transcription in human breast cancer cells. Breast Cancer Res. Treat. 2010, 121, 233-240. [CrossRef] [PubMed]

361. Hou, Y.; Guo, T.; Wu, C.; He, X.; Zhao, M. Effect of shikonin on human breast cancer cells proliferation and apoptosis in vitro. Yakugaku zasshi J. Pharm. Soc. Jpn. 2006, 126, 1383-1386. [CrossRef] [PubMed]

362. Dunai, Z.A.; Imre, G.; Barna, G.; Korcsmaros, T.; Petak, I.; Bauer, P.I.; Mihalik, R. Staurosporine induces necroptotic cell death under caspase-compromised conditions in U937 cells. PLoS ONE 2012, 7, e41945. [CrossRef] [PubMed]

363. Aredia, F.; Scovassi, A.I. Poly(ADP-ribose): A signaling molecule in different paradigms of cell death. Biochem. Pharmacol. 2014, 92, 157-163. [CrossRef]

364. Sosna, J.; Voigt, S.; Mathieu, S.; Lange, A.; Thon, L.; Davarnia, P.; Herdegen, T.; Linkermann, A.; Rittger, A.; Chan, F.K.; et al. TNF-induced necroptosis and PARP-1-mediated necrosis represent distinct routes to programmed necrotic cell death. Cell. Mol. Life Sci. 2014, 71, 331-348. [CrossRef] [PubMed]

365. Basit, F.; Cristofanon, S.; Fulda, S. Obatoclax (GX15-070) triggers necroptosis by promoting the assembly of the necrosome on autophagosomal membranes. Cell Death Differ. 2013, 20, 1161-1173. [CrossRef]

366. Urtishak, K.A.; Edwards, A.Y.; Wang, L.S.; Hudome, A.; Robinson, B.W.; Barrett, J.S.; Cao, K.; Cory, L.; Moore, J.S.; Bantly, A.D.; et al. Potent obatoclax cytotoxicity and activation of triple death mode killing across infant acute lymphoblastic leukemia. Blood 2013, 121, 2689-2703. [CrossRef]

367. Bonapace, L.; Bornhauser, B.C.; Schmitz, M.; Cario, G.; Ziegler, U.; Niggli, F.K.; Schäfer, B.W.; Schrappe, M.; Stanulla, M.; Bourquin, J.P. Induction of autophagy-dependent necroptosis is required for childhood acute lymphoblastic leukemia cells to overcome glucocorticoid resistance. J. Clin. Investig. 2010, 120, 1310-1323. [CrossRef]

368. Coupienne, I.; Bontems, S.; Dewaele, M.; Rubio, N.; Habraken, Y.; Fulda, S.; Agostinis, P.; Piette, J. NF-kappaB inhibition improves the sensitivity of human glioblastoma cells to 5-aminolevulinic acid-based photodynamic therapy. Biochem. Pharmacol. 2011, 81, 606-616. [CrossRef]

369. Möhler, H.; Pfirrmann, R.W.; Frei, K. Redox-directed cancer therapeutics: Taurolidine and Piperlongumine as broadly effective antineoplastic agents (review). Int. J. Oncol. 2014, 45, 1329-1336. [CrossRef]

370. Han, B.; Wang, T.D.; Shen, S.M.; Yu, Y.; Mao, C.; Yao, Z.J.; Wang, L.S. Annonaceous acetogenin mimic AA005 induces cancer cell death via apoptosis inducing factor through a caspase-3-independent mechanism. BMC Cancer 2015, 15, 139. [CrossRef]

371. Longato, G.B.; Fiorito, G.F.; Vendramini-Costa, D.B.; de Oliveira Sousa, I.M.; Tinti, S.V.; Ruiz, A.L.; de Almeida, S.M.; Padilha, R.J.; Foglio, M.A.; de Carvalho, J.E. Different cell death responses induced by eupomatenoid-5 in MCF-7 and 786-0 tumor cell lines. Toxicol. In Vitro 2015, 29, 1026-1033. [CrossRef] [PubMed]

372. Lin, W.; Tongyi, S. Role of Bax/Bcl-2 family members in green tea polyphenol induced necroptosis of p53-deficient Hep3B cells. Tumour Biol. 2014, 35, 8065-8075. [CrossRef] [PubMed]

373. Pozarowski, P.; Halicka, D.H.; Darzynkiewicz, Z. Cell cycle effects and caspase-dependent and independent death of HL-60 and Jurkat cells treated with the inhibitor of NF-kappaB parthenolide. Cell Cycle 2003, 2, 377-383. [CrossRef] [PubMed]

374. Pozarowski, P.; Halicka, D.H.; Darzynkiewicz, Z. NF-kappaB inhibitor sesquiterpene parthenolide induces concurrently atypical apoptosis and cell necrosis: Difficulties in identification of dead cells in such cultures. Cytom. Part A 2003, 54, 118-124. [CrossRef]

375. Lu, C.; Zhou, L.Y.; Xu, H.J.; Chen, X.Y.; Tong, Z.S.; Liu, X.D.; Jia, Y.S.; Chen, Y. RIP3 overexpression sensitizes human breast cancer cells to parthenolide in vitro via intracellular ROS accumulation. Acta Pharmacol. Sin. 2014, 35, 929-936. [CrossRef]

376. Codogno, P.; Meijer, A.J. Atg5: More than an autophagy factor. Nat. Cell Biol. 2006, 8, 1045-1047. [CrossRef] [PubMed]

377. Chen, R.; Wang, H.; Liang, B.; Liu, G.; Tang, M.; Jia, R.; Fan, X.; Jing, W.; Zhou, X.; Wang, H.; et al. Downregulation of ASPP2 improves hepatocellular carcinoma cells survival via promoting BECN1-dependent autophagy initiation. Cell Death Dis. 2016, 7, e2512. [CrossRef]

378. Levine, B.; Kroemer, G. Autophagy in the pathogenesis of disease. Cell 2008, 132, 27-42. [CrossRef] [PubMed]

379. Mizushima, N. The role of the Atg1/ULK1 complex in autophagy regulation. Curr. Opin. Cell Biol. 2010, 22, 132-139. [CrossRef] [PubMed] 
380. Mizushima, N.; Yoshimori, T.; Ohsumi, Y. The role of Atg proteins in autophagosome formation. Annu. Rev. Cell Dev. Biol. 2011, 27, 107-132. [CrossRef] [PubMed]

381. Yang, Z.; Klionsky, D.J. Eaten alive: A history of macroautophagy. Nat. Cell Biol. 2010, 12, 814-822. [CrossRef]

382. Fujii, S.; Mitsunaga, S.; Yamazaki, M.; Hasebe, T.; Ishii, G.; Kojima, M.; Kinoshita, T.; Ueno, T.; Esumi, H.; Ochiai, A. Autophagy is activated in pancreatic cancer cells and correlates with poor patient outcome. Cancer Sci. 2008, 99, 1813-1819. [CrossRef]

383. Sivridis, E.; Koukourakis, M.I.; Zois, C.E.; Ledaki, I.; Ferguson, D.J.; Harris, A.L.; Gatter, K.C.; Giatromanolaki, A. LC3A-positive light microscopy detected patterns of autophagy and prognosis in operable breast carcinomas. Am. J. Pathol. 2010, 176, 2477-2489. [CrossRef]

384. Karpathiou, G.; Sivridis, E.; Koukourakis, M.I.; Mikroulis, D.; Bouros, D.; Froudarakis, M.E.; Giatromanolaki, A. Light-chain 3A autophagic activity and prognostic significance in non-small cell lung carcinomas. Chest 2011, 140, 127-134. [CrossRef]

385. Sivridis, E.; Koukourakis, M.I.; Mendrinos, S.E.; Karpouzis, A.; Fiska, A.; Kouskoukis, C.; Giatromanolaki, A. Beclin-1 and LC3A expression in cutaneous malignant melanomas: A biphasic survival pattern for beclin-1. Melanoma Res. 2011, 21, 188-195. [CrossRef]

386. Yin, Z.; Pascual, C.; Klionsky, D.J. Autophagy: Machinery and regulation. Microb. Cell 2016, 3, 588-596. [CrossRef]

387. Onorati, A.V.; Dyczynski, M.; Ojha, R.; Amaravadi, R.K. Targeting autophagy in cancer. Cancer 2018, 124, 3307-3318. [CrossRef]

388. Saftig, P.; Beertsen, W.; Eskelinen, E.L. LAMP-2: A control step for phagosome and autophagosome maturation. Autophagy 2008, 4, 510-512. [CrossRef]

389. Ravikumar, B.; Sarkar, S.; Davies, J.E.; Futter, M.; Garcia-Arencibia, M.; Green-Thompson, Z.W.; Jimenez-Sanchez, M.; Korolchuk, V.I.; Lichtenberg, M.; Luo, S.; et al. Regulation of mammalian autophagy in physiology and pathophysiology. Physiol. Rev. 2010, 90, 1383-1435. [CrossRef]

390. Mah, L.Y.; Ryan, K.M. Autophagy and cancer. Cold Spring Harb. Perspect. Biol. 2012, 4, a008821. [CrossRef]

391. Levy, J.M.; Thorburn, A. Targeting autophagy during cancer therapy to improve clinical outcomes. Pharmacol. Ther. 2011, 131, 130-141. [CrossRef]

392. Swampillai, A.L.; Salomoni, P.; Short, S.C. The role of autophagy in clinical practice. Clin. Oncol. 2012, 24, 387-395. [CrossRef]

393. Kumar, S.; Gu, Y.; Abudu, Y.P.; Bruun, J.A.; Jain, A.; Farzam, F.; Mudd, M.; Anonsen, J.H.; Rusten, T.E.; Kasof, G.; et al. Phosphorylation of Syntaxin 17 by TBK1 Controls Autophagy Initiation. Dev. Cell 2019, 49, 130-144. [CrossRef]

394. Behrends, C.; Sowa, M.E.; Gygi, S.P.; Harper, J.W. Network organization of the human autophagy system. Nature 2010, 466, 68-76. [CrossRef] [PubMed]

395. Dooley, H.C.; Razi, M.; Polson, H.E.; Girardin, S.E.; Wilson, M.I.; Tooze, S.A. WIPI2 links LC3 conjugation with PI3P, autophagosome formation, and pathogen clearance by recruiting Atg12-5-16L1. Mol. Cell 2014, 55, 238-252. [CrossRef]

396. Walczak, M.; Martens, S. Dissecting the role of the Atg12-Atg5-Atg16 complex during autophagosome formation. Autophagy 2013, 9, 424-425. [CrossRef] [PubMed]

397. Gatica, D.; Lahiri, V.; Klionsky, D.J. Cargo recognition and degradation by selective autophagy. Nat. Cell Biol. 2018, $20,233-242$. [CrossRef] [PubMed]

398. Young, A.R.; Chan, E.Y.; Hu, X.W.; Köchl, R.; Crawshaw, S.G.; High, S.; Hailey, D.W.; Lippincott-Schwartz, J.; Tooze, S.A. Starvation and ULK1-dependent cycling of mammalian Atg9 between the TGN and endosomes. J. Cell Sci. 2006, 119, 3888-3900. [CrossRef]

399. Orsi, A.; Razi, M.; Dooley, H.C.; Robinson, D.; Weston, A.E.; Collinson, L.M.; Tooze, S.A. Dynamic and transient interactions of Atg9 with autophagosomes, but not membrane integration, are required for autophagy. Mol. Biol. Cell 2012, 23, 1860-1873. [CrossRef]

400. Shibutani, S.T.; Yoshimori, T. A current perspective of autophagosome biogenesis. Cell Res. 2014, 24, 58-68. [CrossRef]

401. Nakamura, S.; Yoshimori, T. New insights into autophagosome-lysosome fusion. J. Cell Sci. 2017, 130, 1209-1216. [CrossRef]

402. New, M.; Van Acker, T.; Sakamaki, J.I.; Jiang, M.; Saunders, R.E.; Long, J.; Wang, V.M.; Behrens, A.; Cerveira, J.; Sudhakar, P.; et al. MDH1 and MPP7 Regulate Autophagy in Pancreatic Ductal Adenocarcinoma. Cancer Res. 2019, 79, 1884-1898. [CrossRef] [PubMed]

403. Levy, J.M.M.; Thorburn, A. Autophagy in cancer: Moving from understanding mechanism to improving therapy responses in patients. Cell Death Differ. 2020, 27, 843-857. [CrossRef] [PubMed]

404. Kiruthiga, C.; Devi, K.P.; Nabavi, S.M.; Bishayee, A. Autophagy: A Potential Therapeutic Target of Polyphenols in Hepatocellular Carcinoma. Cancers 2020, 12, 562. [CrossRef] [PubMed]

405. Rao, S.; Yang, H.; Penninger, J.M.; Kroemer, G. Autophagy in non-small cell lung carcinogenesis: A positive regulator of antitumor immunosurveillance. Autophagy 2014, 10, 529-531. [CrossRef]

406. Ma, Y.; Galluzzi, L.; Zitvogel, L.; Kroemer, G. Autophagy and cellular immune responses. Immunity 2013, 39, 211-227. [CrossRef]

407. Townsend, K.N.; Hughson, L.R.; Schlie, K.; Poon, V.I.; Westerback, A.; Lum, J.J. Autophagy inhibition in cancer therapy: Metabolic considerations for antitumor immunity. Immunol. Rev. 2012, 249, 176-194. [CrossRef]

408. Ko, A.; Kanehisa, A.; Martins, I.; Senovilla, L.; Chargari, C.; Dugue, D.; Mariño, G.; Kepp, O.; Michaud, M.; Perfettini, J.L.; et al. Autophagy inhibition radiosensitizes in vitro, yet reduces radioresponses in vivo due to deficient immunogenic signalling. Cell Death Differ. 2014, 21, 92-99. [CrossRef] 
409. Michaud, M.; Martins, I.; Sukkurwala, A.Q.; Adjemian, S.; Ma, Y.; Pellegatti, P.; Shen, S.; Kepp, O.; Scoazec, M.; Mignot, G.; et al. Autophagy-dependent anticancer immune responses induced by chemotherapeutic agents in mice. Science 2011, 334, $1573-1577$. [CrossRef]

410. Lechner, M.G.; Karimi, S.S.; Barry-Holson, K.; Angell, T.E.; Murphy, K.A.; Church, C.H.; Ohlfest, J.R.; Hu, P.; Epstein, A.L. Immunogenicity of murine solid tumor models as a defining feature of in vivo behavior and response to immunotherapy. $J$. Immunother. 2013, 36, 477-489. [CrossRef] [PubMed]

411. Starobinets, H.; Ye, J.; Broz, M.; Barry, K.; Goldsmith, J.; Marsh, T.; Rostker, F.; Krummel, M.; Debnath, J. Antitumor adaptive immunity remains intact following inhibition of autophagy and antimalarial treatment. J. Clin. Investig. 2016, 126, 4417-4429. [CrossRef] [PubMed]

412. Pietrocola, F.; Pol, J.; Vacchelli, E.; Rao, S.; Enot, D.P.; Baracco, E.E.; Levesque, S.; Castoldi, F.; Jacquelot, N.; Yamazaki, T.; et al. Caloric Restriction Mimetics Enhance Anticancer Immunosurveillance. Cancer Cell 2016, 30, 147-160. [CrossRef] [PubMed]

413. Levine, B.; Klionsky, D.J. Autophagy wins the 2016 Nobel Prize in Physiology or Medicine: Breakthroughs in baker's yeast fuel advances in biomedical research. Proc. Natl. Acad. Sci. USA 2017, 114, 201-205. [CrossRef]

414. Li, Y.; Hahn, T.; Garrison, K.; Cui, Z.H.; Thorburn, A.; Thorburn, J.; Hu, H.M.; Akporiaye, E.T. The vitamin E analogue $\alpha$-TEA stimulates tumor autophagy and enhances antigen cross-presentation. Cancer Res. 2012, 72, 3535-3545. [CrossRef]

415. Baginska, J.; Viry, E.; Berchem, G.; Poli, A.; Noman, M.Z.; van Moer, K.; Medves, S.; Zimmer, J.; Oudin, A.; Niclou, S.P.; et al. Granzyme B degradation by autophagy decreases tumor cell susceptibility to natural killer-mediated lysis under hypoxia. Proc. Natl. Acad. Sci. USA 2013, 110, 17450-17455. [CrossRef]

416. Liang, X.; De Vera, M.E.; Buchser, W.J.; Romo de Vivar Chavez, A.; Loughran, P.; Beer Stolz, D.; Basse, P.; Wang, T.; Van Houten, B.; Zeh, H.J., 3rd; et al. Inhibiting systemic autophagy during interleukin 2 immunotherapy promotes long-term tumor regression. Cancer Res. 2012, 72, 2791-2801. [CrossRef]

417. Levy, J.M.M.; Towers, C.G.; Thorburn, A. Targeting autophagy in cancer. Nat. Rev. Cancer 2017, 17, 528-542. [CrossRef]

418. Page, D.B.; Hulett, T.W.; Hilton, T.L.; Hu, H.M.; Urba, W.J.; Fox, B.A. Glimpse into the future: Harnessing autophagy to promote anti-tumor immunity with the DRibbles vaccine. J. Immunother. Cancer 2016, 4, 25. [CrossRef]

419. Hilton, T.; Sanborn, R.; Boulmay, B.; Li, R.; Spieler, B.; Happel, K.; Paustian, C.; Moudgil, T.; Dubay, C.; Fisher, B.; et al. Preliminary analysis of immune responses in patients enrolled in a Phase II trial of cyclophosphamide with allogenic dribble vaccine alone (DPV-001) or with GM-CSF or imiquimod for adjuvant treatment of stage IIIa or IIIb NSCLC. J. ImmunoTherapy Cancer 2014, 2, P249. [CrossRef]

420. Karsli-Uzunbas, G.; Guo, J.Y.; Price, S.; Teng, X.; Laddha, S.V.; Khor, S.; Kalaany, N.Y.; Jacks, T.; Chan, C.S.; Rabinowitz, J.D.; et al. Autophagy is required for glucose homeostasis and lung tumor maintenance. Cancer Discov. 2014, 4, 914-927. [CrossRef]

421. Levy, J.M.; Thompson, J.C.; Griesinger, A.M.; Amani, V.; Donson, A.M.; Birks, D.K.; Morgan, M.J.; Mirsky, D.M.; Handler, M.H.; Foreman, N.K.; et al. Autophagy inhibition improves chemosensitivity in BRAF(V600E) brain tumors. Cancer Discov. 2014, 4, 773-780. [CrossRef]

422. Chhipa, R.R.; Wu, Y.; Ip, C. AMPK-mediated autophagy is a survival mechanism in androgen-dependent prostate cancer cells subjected to androgen deprivation and hypoxia. Cell. Signal. 2011, 23, 1466-1472. [CrossRef]

423. Han, W.; Pan, H.; Chen, Y.; Sun, J.; Wang, Y.; Li, J.; Ge, W.; Feng, L.; Lin, X.; Wang, X.; et al. EGFR tyrosine kinase inhibitors activate autophagy as a cytoprotective response in human lung cancer cells. PLoS ONE 2011, 6, e18691. [CrossRef]

424. Hou, Y.J.; Dong, L.W.; Tan, Y.X.; Yang, G.Z.; Pan, Y.F.; Li, Z.; Tang, L.; Wang, M.; Wang, Q.; Wang, H.Y. Inhibition of active autophagy induces apoptosis and increases chemosensitivity in cholangiocarcinoma. Lab. Investig. 2011, 91, 1146-1157. [CrossRef] [PubMed]

425. Mukubou, H.; Tsujimura, T.; Sasaki, R.; Ku, Y. The role of autophagy in the treatment of pancreatic cancer with gemcitabine and ionizing radiation. Int. J. Oncol. 2010, 37, 821-828. [CrossRef] [PubMed]

426. Shi, Y.H.; Ding, Z.B.; Zhou, J.; Hui, B.; Shi, G.M.; Ke, A.W.; Wang, X.Y.; Dai, Z.; Peng, Y.F.; Gu, C.Y.; et al. Targeting autophagy enhances sorafenib lethality for hepatocellular carcinoma via ER stress-related apoptosis. Autophagy 2011, 7, 1159-1172. [CrossRef]

427. Sun, W.L.; Chen, J.; Wang, Y.P.; Zheng, H. Autophagy protects breast cancer cells from epirubicin-induced apoptosis and facilitates epirubicin-resistance development. Autophagy 2011, 7, 1035-1044. [CrossRef] [PubMed]

428. Xu, L.; Qu, X.J.; Liu, Y.P.; Xu, Y.Y.; Liu, J.; Hou, K.Z.; Zhang, Y. Protective autophagy antagonizes oxaliplatin-induced apoptosis in gastric cancer cells. Chin. J. Cancer 2011, 30, 490-496. [CrossRef]

429. Tan, Q.; Joshua, A.M.; Wang, M.; Bristow, R.G.; Wouters, B.G.; Allen, C.J.; Tannock, I.F. Up-regulation of autophagy is a mechanism of resistance to chemotherapy and can be inhibited by pantoprazole to increase drug sensitivity. Cancer Chemother. Pharmacol. 2017, 79, 959-969. [CrossRef] [PubMed]

430. Tan, Q.; Wang, M.; Yu, M.; Zhang, J.; Bristow, R.G.; Hill, R.P.; Tannock, I.F. Role of Autophagy as a Survival Mechanism for Hypoxic Cells in Tumors. Neoplasia 2016, 18, 347-355. [CrossRef] [PubMed]

431. Borst, P.; Rottenberg, S.; Jonkers, J. How do real tumors become resistant to cisplatin? Cell Cycle 2008, 7, 1353-1359. [CrossRef] [PubMed]

432. Rebecca, V.W.; Amaravadi, R.K. Emerging strategies to effectively target autophagy in cancer. Oncogene 2016, 35, 1-11. [CrossRef] [PubMed]

433. Pandey, S.; Chandravati, C. Autophagy in cervical cancer: An emerging therapeutic target. Asian Pac. J. Cancer Prev. 2012, 13, 4867-4871. [CrossRef] [PubMed] 
434. Rikiishi, H. Autophagic action of new targeting agents in head and neck oncology. Cancer Biol. Ther. 2012, 13, 978-991. [CrossRef]

435. Ma, X.H.; Piao, S.F.; Dey, S.; McAfee, Q.; Karakousis, G.; Villanueva, J.; Hart, L.S.; Levi, S.; Hu, J.; Zhang, G.; et al. Targeting ER stress-induced autophagy overcomes BRAF inhibitor resistance in melanoma. J. Clin. Investig. 2014, 124, 1406-1417. [CrossRef]

436. Zhang, S.F.; Wang, X.Y.; Fu, Z.Q.; Peng, Q.H.; Zhang, J.Y.; Ye, F.; Fu, Y.F.; Zhou, C.Y.; Lu, W.G.; Cheng, X.D.; et al. TXNDC17 promotes paclitaxel resistance via inducing autophagy in ovarian cancer. Autophagy 2015, 11, 225-238. [CrossRef]

437. Wu, H.M.; Jiang, Z.F.; Ding, P.S.; Shao, L.J.; Liu, R.Y. Hypoxia-induced autophagy mediates cisplatin resistance in lung cancer cells. Sci. Rep. 2015, 5, 12291. [CrossRef]

438. Mahoney, E.; Lucas, D.M.; Gupta, S.V.; Wagner, A.J.; Herman, S.E.; Smith, L.L.; Yeh, Y.Y.; Andritsos, L.; Jones, J.A.; Flynn, J.M.; et al. ER stress and autophagy: New discoveries in the mechanism of action and drug resistance of the cyclin-dependent kinase inhibitor flavopiridol. Blood 2012, 120, 1262-1273. [CrossRef]

439. Li, Z.Y.; Zhang, C.; Zhang, Y.; Chen, L.; Chen, B.D.; Li, Q.Z.; Zhang, X.J.; Li, W.P. A novel HDAC6 inhibitor Tubastatin A: Controls HDAC6-p97/VCP-mediated ubiquitination-autophagy turnover and reverses Temozolomide-induced ER stress-tolerance in GBM cells. Cancer Lett. 2017, 391, 89-99. [CrossRef]

440. Aveic, S.; Tonini, G.P. Resistance to receptor tyrosine kinase inhibitors in solid tumors: Can we improve the cancer fighting strategy by blocking autophagy? Cancer Cell Int. 2016, 16, 62. [CrossRef]

441. Chen, S.; Rehman, S.K.; Zhang, W.; Wen, A.; Yao, L.; Zhang, J. Autophagy is a therapeutic target in anticancer drug resistance. Biochim. Biophys. Acta 2010, 1806, 220-229. [CrossRef]

442. Kumar, A.; Singh, U.K.; Chaudhary, A. Targeting autophagy to overcome drug resistance in cancer therapy. Future Med. Chem. 2015, 7, 1535-1542. [CrossRef]

443. Sannigrahi, M.K.; Singh, V.; Sharma, R.; Panda, N.K.; Khullar, M. Role of autophagy in head and neck cancer and therapeutic resistance. Oral Dis. 2015, 21, 283-291. [CrossRef] [PubMed]

444. Sui, X.; Chen, R.; Wang, Z.; Huang, Z.; Kong, N.; Zhang, M.; Han, W.; Lou, F.; Yang, J.; Zhang, Q.; et al. Autophagy and chemotherapy resistance: A promising therapeutic target for cancer treatment. Cell Death Dis. 2013, 4, e838. [CrossRef]

445. Viale, A.; Pettazzoni, P.; Lyssiotis, C.A.; Ying, H.; Sánchez, N.; Marchesini, M.; Carugo, A.; Green, T.; Seth, S.; Giuliani, V.; et al. Oncogene ablation-resistant pancreatic cancer cells depend on mitochondrial function. Nature 2014, 514, 628-632. [CrossRef]

446. Katheder, N.S.; Khezri, R.; O’Farrell, F.; Schultz, S.W.; Jain, A.; Rahman, M.M.; Schink, K.O.; Theodossiou, T.A.; Johansen, T.; Juhász, G.; et al. Microenvironmental autophagy promotes tumour growth. Nature 2017, 541, 417-420. [CrossRef] [PubMed]

447. Langie, S.A.; Koppen, G.; Desaulniers, D.; Al-Mulla, F.; Al-Temaimi, R.; Amedei, A.; Azqueta, A.; Bisson, W.H.; Brown, D.G.; Brunborg, G.; et al. Causes of genome instability: The effect of low dose chemical exposures in modern society. Carcinogenesis 2015, 36 (Suppl. 1), S61-S88. [CrossRef] [PubMed]

448. Mohammad, R.M.; Muqbil, I.; Lowe, L.; Yedjou, C.; Hsu, H.Y.; Lin, L.T.; Siegelin, M.D.; Fimognari, C.; Kumar, N.B.; Dou, Q.P.; et al. Broad targeting of resistance to apoptosis in cancer. Semin. Cancer Biol. 2015, 35, S78-S103. [CrossRef] [PubMed]

449. Wang, X.; Feng, Y.; Wang, N.; Cheung, F.; Tan, H.Y.; Zhong, S.; Li, C.; Kobayashi, S. Chinese medicines induce cell death: The molecular and cellular mechanisms for cancer therapy. BioMed Res. Int. 2014, 2014, 530342. [CrossRef] [PubMed]

450. Zhou, M.; Wang, R. Small-molecule regulators of autophagy and their potential therapeutic applications. ChemMedChem 2013, 8 , 694-707. [CrossRef]

451. Sun, H.; Wang, Z.; Yakisich, J.S. Natural products targeting autophagy via the PI3K/Akt/mTOR pathway as anticancer agents. Anti-Cancer Agents Med. Chem. 2013, 13, 1048-1056. [CrossRef]

452. Mukhtar, E.; Adhami, V.M.; Khan, N.; Mukhtar, H. Apoptosis and autophagy induction as mechanism of cancer prevention by naturally occurring dietary agents. Curr. Drug Targets 2012, 13, 1831-1841. [CrossRef]

453. Zhang, X.; Chen, L.X.; Ouyang, L.; Cheng, Y.; Liu, B. Plant natural compounds: Targeting pathways of autophagy as anti-cancer therapeutic agents. Cell Prolif. 2012, 45, 466-476. [CrossRef] [PubMed]

454. Hsieh, M.-J.; Lin, C.-W.; Su, S.-C.; Reiter, R.J.; Chen, A.W.-G.; Chen, M.-K.; Yang, S.-F. Effects of miR-34b/miR-892a Upregulation and Inhibition of ABCB1/ABCB4 on Melatonin-Induced Apoptosis in VCR-Resistant Oral Cancer Cells. Mol. Ther. Nucleic Acids 2020, 19, 877-889. [CrossRef]

455. Singletary, K.; Milner, J. Diet, autophagy, and cancer: A review. Cancer Epidemiol. Biomark. Prev. 2008, 17, 1596-1610. [CrossRef] [PubMed]

456. Akar, U.; Ozpolat, B.; Mehta, K.; Fok, J.; Kondo, Y.; Lopez-Berestein, G. Tissue transglutaminase inhibits autophagy in pancreatic cancer cells. Mol. Cancer Res. 2007, 5, 241-249. [CrossRef]

457. Shinojima, N.; Yokoyama, T.; Kondo, Y.; Kondo, S. Roles of the Akt/mTOR/p70S6K and ERK1/2 signaling pathways in curcumin-induced autophagy. Autophagy 2007, 3, 635-637. [CrossRef]

458. Opipari, A.W., Jr.; Tan, L.; Boitano, A.E.; Sorenson, D.R.; Aurora, A.; Liu, J.R. Resveratrol-induced autophagocytosis in ovarian cancer cells. Cancer Res. 2004, 64, 696-703. [CrossRef] [PubMed]

459. Gordon, P.B.; Holen, I.; Seglen, P.O. Protection by naringin and some other flavonoids of hepatocytic autophagy and endocytosis against inhibition by okadaic acid. J. Biol. Chem. 1995, 270, 5830-5838. [CrossRef]

460. Blankson, H.; Holen, I.; Seglen, P.O. Disruption of the cytokeratin cytoskeleton and inhibition of hepatocytic autophagy by okadaic acid. Exp. Cell Res. 1995, 218, 522-530. [CrossRef] [PubMed] 
461. Psahoulia, F.H.; Moumtzi, S.; Roberts, M.L.; Sasazuki, T.; Shirasawa, S.; Pintzas, A. Quercetin mediates preferential degradation of oncogenic Ras and causes autophagy in Ha-RAS-transformed human colon cells. Carcinogenesis 2007, 28, 1021-1031. [CrossRef] [PubMed]

462. Tan, W.; Lu, J.; Huang, M.; Li, Y.; Chen, M.; Wu, G.; Gong, J.; Zhong, Z.; Xu, Z.; Dang, Y.; et al. Anti-cancer natural products isolated from chinese medicinal herbs. Chin. Med. 2011, 6, 27. [CrossRef] [PubMed]

463. Aiyer, H.S.; Warri, A.M.; Woode, D.R.; Hilakivi-Clarke, L.; Clarke, R. Influence of berry polyphenols on receptor signaling and cell-death pathways: Implications for breast cancer prevention. J. Agric. Food Chem. 2012, 60, 5693-5708. [CrossRef] [PubMed]

464. Gao, A.M.; Zhang, X.Y.; Hu, J.N.; Ke, Z.P. Apigenin sensitizes hepatocellular carcinoma cells to doxorubic through regulating miR-520b/ATG7 axis. Chem. Biol. Interact. 2018, 280, 45-50. [CrossRef] [PubMed]

465. Liu, Q.; Zhu, D.; Hao, B.; Zhang, Z.; Tian, Y. Luteolin promotes the sensitivity of cisplatin in ovarian cancer by decreasing PRPA1-medicated autophagy. Cell. Mol. Biol. 2018, 64, 17-22. [CrossRef]

466. Fan, S.; Li, L.; Chen, S.; Yu, Y.; Qi, M.; Tashiro, S.; Onodera, S.; Ikejima, T. Silibinin induced-autophagic and apoptotic death is associated with an increase in reactive oxygen and nitrogen species in HeLa cells. Free. Radic. Res. 2011, 45, 1307-1324. [CrossRef]

467. Jiang, K.; Wang, W.; Jin, X.; Wang, Z.; Ji, Z.; Meng, G. Silibinin, a natural flavonoid, induces autophagy via ROS-dependent mitochondrial dysfunction and loss of ATP involving BNIP3 in human MCF7 breast cancer cells. Oncol. Rep. 2015, 33, 2711-2718. [CrossRef]

468. Duan, W.J.; Li, Q.S.; Xia, M.Y.; Tashiro, S.; Onodera, S.; Ikejima, T. Silibinin activated p53 and induced autophagic death in human fibrosarcoma HT1080 cells via reactive oxygen species-p38 and c-Jun N-terminal kinase pathways. Biol. Pharm. Bull. 2011, 34, 47-53. [CrossRef]

469. Jiang, Y.Y.; Yang, R.; Wang, H.J.; Huang, H.; Wu, D.; Tashiro, S.; Onodera, S.; Ikejima, T. Mechanism of autophagy induction and role of autophagy in antagonizing mitomycin C-induced cell apoptosis in silibinin treated human melanoma A375-S2 cells. Eur. J. Pharmacol. 2011, 659, 7-14. [CrossRef]

470. Kauntz, H.; Bousserouel, S.; Gossé, F.; Raul, F. Silibinin triggers apoptotic signaling pathways and autophagic survival response in human colon adenocarcinoma cells and their derived metastatic cells. Apoptosis 2011, 16, 1042-1053. [CrossRef]

471. Wang, L.; Zhu, Z.; Han, L.; Zhao, L.; Weng, J.; Yang, H.; Wu, S.; Chen, K.; Wu, L.; Chen, T. A curcumin derivative, WZ35, suppresses hepatocellular cancer cell growth via downregulating YAP-mediated autophagy. Food Funct. 2019, 10, 3748-3757. [CrossRef]

472. Rainey, N.; Motte, L.; Aggarwal, B.B.; Petit, P.X. Curcumin hormesis mediates a cross-talk between autophagy and cell death. Cell Death Dis. 2015, 6, e2003. [CrossRef]

473. Elmansi, A.M.; El-Karef, A.A.; Shishtawy, M.; Eissa, L.A. Hepatoprotective Effect of Curcumin on Hepatocellular Carcinoma Through Autophagic and Apoptic Pathways. Ann. Hepatol. 2017, 16, 607-618. [CrossRef]

474. Qian, H.; Yang, Y.; Wang, X. Curcumin enhanced adriamycin-induced human liver-derived Hepatoma G2 cell death through activation of mitochondria-mediated apoptosis and autophagy. Eur. J. Pharm. Sci. 2011, 43, 125-131. [CrossRef] [PubMed]

475. Maioli, E.; Greci, L.; Soucek, K.; Hyzdalova, M.; Pecorelli, A.; Fortino, V.; Valacchi, G. Rottlerin inhibits ROS formation and prevents NFkappaB activation in MCF-7 and HT-29 cells. J. Biomed. Biotechnol. 2009, 2009, 742936. [CrossRef] [PubMed]

476. Kumar, D.; Shankar, S.; Srivastava, R.K. Rottlerin induces autophagy and apoptosis in prostate cancer stem cells via PI3K/Akt/mTOR signaling pathway. Cancer Lett. 2014, 343, 179-189. [CrossRef] [PubMed]

477. Lu, Q.Y.; Zhang, L.; Lugea, A.; Moro, A.; Edderkaoui, M.; Eibl, G.; Pandol, S.J.; Go, V.L. Determination of Rottlerin, a Natural Protein Kinases C Inhibitor, in Pancreatic Cancer Cells and Mouse Xenografts by RP-HPLC Method. J. Chromatogr. Sep. Tech. 2013, 4, 100062.

478. Yu, Z.; Li, W.; Liu, F. Inhibition of proliferation and induction of apoptosis by genistein in colon cancer HT-29 cells. Cancer Lett. 2004, 215, 159-166. [CrossRef] [PubMed]

479. Gossner, G.; Choi, M.; Tan, L.; Fogoros, S.; Griffith, K.A.; Kuenker, M.; Liu, J.R. Genistein-induced apoptosis and autophagocytosis in ovarian cancer cells. Gynecol. Oncol. 2007, 105, 23-30. [CrossRef] [PubMed]

480. Hasima, N.; Ozpolat, B. Regulation of autophagy by polyphenolic compounds as a potential therapeutic strategy for cancer. Cell Death Dis. 2014, 5, e1509. [CrossRef] [PubMed]

481. Yang, Z.; Kulkarni, K.; Zhu, W.; Hu, M. Bioavailability and pharmacokinetics of genistein: Mechanistic studies on its ADME. Anti-Cancer Agents Med. Chem. 2012, 12, 1264-1280. [CrossRef] [PubMed]

482. Ranelletti, F.O.; Maggiano, N.; Serra, F.G.; Ricci, R.; Larocca, L.M.; Lanza, P.; Scambia, G.; Fattorossi, A.; Capelli, A.; Piantelli, M. Quercetin inhibits p21-RAS expression in human colon cancer cell lines and in primary colorectal tumors. Int. J. Cancer 2000, 85, 438-445. [CrossRef]

483. Wang, K.; Liu, R.; Li, J.; Mao, J.; Lei, Y.; Wu, J.; Zeng, J.; Zhang, T.; Wu, H.; Chen, L.; et al. Quercetin induces protective autophagy in gastric cancer cells: Involvement of Akt-mTOR- and hypoxia-induced factor 1 $\alpha$-mediated signaling. Autophagy 2011, 7, 966-978. [CrossRef]

484. Martinez-Outschoorn, U.E.; Balliet, R.M.; Rivadeneira, D.B.; Chiavarina, B.; Pavlides, S.; Wang, C.; Whitaker-Menezes, D.; Daumer, K.M.; Lin, Z.; Witkiewicz, A.K.; et al. Oxidative stress in cancer associated fibroblasts drives tumor-stroma co-evolution: A new paradigm for understanding tumor metabolism, the field effect and genomic instability in cancer cells. Cell Cycle 2010, 9, 3256-3276. [CrossRef] [PubMed] 
485. Liao, P.C.; Ng, L.T.; Lin, L.T.; Richardson, C.D.; Wang, G.H.; Lin, C.C. Resveratrol arrests cell cycle and induces apoptosis in human hepatocellular carcinoma Huh-7 cells. J. Med. Food 2010, 13, 1415-1423. [CrossRef]

486. Guamán Ortiz, L.M.; Tillhon, M.; Parks, M.; Dutto, I.; Prosperi, E.; Savio, M.; Arcamone, A.G.; Buzzetti, F.; Lombardi, P.; Scovassi, A.I. Multiple effects of berberine derivatives on colon cancer cells. BioMed Res. Int. 2014, 2014, 924585. [CrossRef] [PubMed]

487. Zhang, B.; Yin, X.; Sui, S. Resveratrol inhibited the progression of human hepatocellular carcinoma by inducing autophagy via regulating p53 and the phosphoinositide 3-kinase/protein kinase B pathway. Oncol. Rep. 2018, 40, 2758-2765. [CrossRef]

488. Puissant, A.; Robert, G.; Fenouille, N.; Luciano, F.; Cassuto, J.P.; Raynaud, S.; Auberger, P. Resveratrol promotes autophagic cell death in chronic myelogenous leukemia cells via JNK-mediated p62/SQSTM1 expression and AMPK activation. Cancer Res. 2010, 70, 1042-1052. [CrossRef]

489. Kueck, A.; Opipari, A.W., Jr.; Griffith, K.A.; Tan, L.; Choi, M.; Huang, J.; Wahl, H.; Liu, J.R. Resveratrol inhibits glucose metabolism in human ovarian cancer cells. Gynecol. Oncol. 2007, 107, 450-457. [CrossRef] [PubMed]

490. Trincheri, N.F.; Follo, C.; Nicotra, G.; Peracchio, C.; Castino, R.; Isidoro, C. Resveratrol-induced apoptosis depends on the lipid kinase activity of Vps34 and on the formation of autophagolysosomes. Carcinogenesis 2008, 29, 381-389. [CrossRef] [PubMed]

491. Li, J.; Qin, Z.; Liang, Z. The prosurvival role of autophagy in Resveratrol-induced cytotoxicity in human U251 glioma cells. BMC Cancer 2009, 9, 215. [CrossRef] [PubMed]

492. Chen, T.C.; Hung, Y.C.; Lin, T.Y.; Chang, H.W.; Chiang, I.P.; Chen, Y.Y.; Chow, K.C. Human papillomavirus infection and expression of ATPase family AAA domain containing 3A, a novel anti-autophagy factor, in uterine cervical cancer. Int. J. Mol. Med. 2011, 28, 689-696. [CrossRef]

493. Selvaraj, S.; Sun, Y.; Sukumaran, P.; Singh, B.B. Resveratrol activates autophagic cell death in prostate cancer cells via downregulation of STIM1 and the mTOR pathway. Mol. Carcinog. 2016, 55, 818-831. [CrossRef] [PubMed]

494. Lang, F.; Qin, Z.; Li, F.; Zhang, H.; Fang, Z.; Hao, E. Apoptotic Cell Death Induced by Resveratrol Is Partially Mediated by the Autophagy Pathway in Human Ovarian Cancer Cells. PLoS ONE 2015, 10, e0129196. [CrossRef]

495. Fu, Y.; Chang, H.; Peng, X.; Bai, Q.; Yi, L.; Zhou, Y.; Zhu, J.; Mi, M. Resveratrol inhibits breast cancer stem-like cells and induces autophagy via suppressing Wnt/ $\beta$-catenin signaling pathway. PLoS ONE 2014, 9, e102535. [CrossRef]

496. Ge, J.; Liu, Y.; Li, Q.; Guo, X.; Gu, L.; Ma, Z.G.; Zhu, Y.P. Resveratrol induces apoptosis and autophagy in T-cell acute lymphoblastic leukemia cells by inhibiting Akt/mTOR and activating p38-MAPK. Biomed. Environ. Sci. 2013, 26, 902-911. [CrossRef]

497. Li, G.; Rivas, P.; Bedolla, R.; Thapa, D.; Reddick, R.L.; Ghosh, R.; Kumar, A.P. Dietary resveratrol prevents development of high-grade prostatic intraepithelial neoplastic lesions: Involvement of SIRT1/S6K axis. Cancer Prev. Res. 2013, 6, 27-39. [CrossRef]

498. Talib, W.H.; Alsayed, A.R.; Farhan, F.; Al Kury, L.T. Resveratrol and tumor microenvironment: Mechanistic basis and therapeutic targets. Molecules 2020, 25, 4282. [CrossRef]

499. Feng, R.; Wang, S.Y.; Shi, Y.H.; Fan, J.; Yin, X.M. Delphinidin induces necrosis in hepatocellular carcinoma cells in the presence of 3-methyladenine, an autophagy inhibitor. J. Agric. Food Chem. 2010, 58, 3957-3964. [CrossRef]

500. Cheng, X.; Zhong, F.; He, K.; Sun, S.; Chen, H.; Zhou, J. EHHM, a novel phenolic natural product from Livistona chinensis, induces autophagy-related apoptosis in hepatocellular carcinoma cells. Oncol. Lett. 2016, 12, 3739-3748. [CrossRef] [PubMed]

501. Wang, N.; Feng, Y.; Zhu, M.; Tsang, C.M.; Man, K.; Tong, Y.; Tsao, S.W. Berberine induces autophagic cell death and mitochondrial apoptosis in liver cancer cells: The cellular mechanism. J. Cell. Biochem. 2010, 111, 1426-1436. [CrossRef] [PubMed]

502. Peng, P.L.; Kuo, W.H.; Tseng, H.C.; Chou, F.P. Synergistic tumor-killing effect of radiation and berberine combined treatment in lung cancer: The contribution of autophagic cell death. Int. J. Radiat. Oncol. Biol. Phys. 2008, 70, 529-542. [CrossRef]

503. Ortiz, L.M.; Lombardi, P.; Tillhon, M.; Scovassi, A.I. Berberine, an epiphany against cancer. Molecules 2014, 19, 12349-12367. [CrossRef] [PubMed]

504. Guamán Ortiz, L.M.; Croce, A.L.; Aredia, F.; Sapienza, S.; Fiorillo, G.; Syeda, T.M.; Buzzetti, F.; Lombardi, P.; Scovassi, A.I. Effect of new berberine derivatives on colon cancer cells. Acta Biochim. Biophys. Sin. 2015, 47, 824-833. [CrossRef] [PubMed]

505. Tillhon, M.; Guamán Ortiz, L.M.; Lombardi, P.; Scovassi, A.I. Berberine: New perspectives for old remedies. Biochem. Pharmacol. 2012, 84, 1260-1267. [CrossRef]

506. Aredia, F.; Guamán Ortiz, L.M.; Giansanti, V.; Scovassi, A.I. Autophagy and cancer. Cells 2012, 1, 520-534. [CrossRef]

507. Aras, A.; Khokhar, A.R.; Qureshi, M.Z.; Silva, M.F.; Sobczak-Kupiec, A.; Pineda, E.A.; Hechenleitner, A.A.; Farooqi, A.A. Targeting cancer with nano-bullets: Curcumin, EGCG, resveratrol and quercetin on flying carpets. Asian Pac. J. Cancer Prev. 2014, 15, 3865-3871. [CrossRef]

508. Siddiqui, I.A.; Malik, A.; Adhami, V.M.; Asim, M.; Hafeez, B.B.; Sarfaraz, S.; Mukhtar, H. Green tea polyphenol EGCG sensitizes human prostate carcinoma LNCaP cells to TRAIL-mediated apoptosis and synergistically inhibits biomarkers associated with angiogenesis and metastasis. Oncogene 2008, 27, 2055-2063. [CrossRef]

509. Tang, S.N.; Singh, C.; Nall, D.; Meeker, D.; Shankar, S.; Srivastava, R.K. The dietary bioflavonoid quercetin synergizes with epigallocathechin gallate (EGCG) to inhibit prostate cancer stem cell characteristics, invasion, migration and epithelial-mesenchymal transition. J. Mol. Signal. 2010, 5, 14. [CrossRef] [PubMed]

510. Hu, F.; Wei, F.; Wang, Y.; Wu, B.; Fang, Y.; Xiong, B. EGCG synergizes the therapeutic effect of cisplatin and oxaliplatin through autophagic pathway in human colorectal cancer cells. J. Pharmacol. Sci. 2015, 128, 27-34. [CrossRef] [PubMed]

511. Zhou, J.; Farah, B.L.; Sinha, R.A.; Wu, Y.; Singh, B.K.; Bay, B.H.; Yang, C.S.; Yen, P.M. Epigallocatechin-3-gallate (EGCG), a green tea polyphenol, stimulates hepatic autophagy and lipid clearance. PLoS ONE 2014, 9, e87161. [CrossRef] [PubMed] 
512. Chen, L.; Ye, H.L.; Zhang, G.; Yao, W.M.; Chen, X.Z.; Zhang, F.C.; Liang, G. Autophagy inhibition contributes to the synergistic interaction between EGCG and doxorubicin to kill the hepatoma Hep3B cells. PLoS ONE 2014, 9, e85771. [CrossRef]

513. Satoh, M.; Takemura, Y.; Hamada, H.; Sekido, Y.; Kubota, S. EGCG induces human mesothelioma cell death by inducing reactive oxygen species and autophagy. Cancer Cell Int. 2013, 13, 19. [CrossRef]

514. Zhang, Y.; Yang, N.D.; Zhou, F.; Shen, T.; Duan, T.; Zhou, J.; Shi, Y.; Zhu, X.Q.; Shen, H.M. (-)-Epigallocatechin-3-gallate induces non-apoptotic cell death in human cancer cells via ROS-mediated lysosomal membrane permeabilization. PLoS ONE 2012, 7, e46749. [CrossRef]

515. Boyanapalli, S.S.; Kong, A.T. "Curcumin, the King of Spices": Epigenetic Regulatory Mechanisms in the Prevention of Cancer, Neurological, and Inflammatory Diseases. Curr. Pharmacol. Rep. 2015, 1, 129-139. [CrossRef]

516. Aoki, H.; Takada, Y.; Kondo, S.; Sawaya, R.; Aggarwal, B.B.; Kondo, Y. Evidence that curcumin suppresses the growth of malignant gliomas in vitro and in vivo through induction of autophagy: Role of Akt and extracellular signal-regulated kinase signaling pathways. Mol. Pharmacol. 2007, 72, 29-39. [CrossRef]

517. Jia, Y.L.; Li, J.; Qin, Z.H.; Liang, Z.Q. Autophagic and apoptotic mechanisms of curcumin-induced death in K562 cells. J. Asian Nat. Prod. Res. 2009, 11, 918-928. [CrossRef]

518. Zhou, D.Y.; Ding, N.; Du, Z.Y.; Cui, X.X.; Wang, H.; Wei, X.C.; Conney, A.H.; Zhang, K.; Zheng, X. Curcumin analogues with high activity for inhibiting human prostate cancer cell growth and androgen receptor activation. Mol. Med. Rep. 2014, 10, 1315-1322. [CrossRef] [PubMed]

519. Akkoç, Y.; Berrak, Ö.; Arısan, E.D.; Obakan, P.; Çoker-Gürkan, A.; Palavan-Ünsal, N. Inhibition of PI3K signaling triggered apoptotic potential of curcumin which is hindered by Bcl-2 through activation of autophagy in MCF-7 cells. Biomed. Pharmacother. 2015, 71, 161-171. [CrossRef]

520. Xiao, K.; Jiang, J.; Guan, C.; Dong, C.; Wang, G.; Bai, L.; Sun, J.; Hu, C.; Bai, C. Curcumin induces autophagy via activating the AMPK signaling pathway in lung adenocarcinoma cells. J. Pharmacol. Sci. 2013, 123, 102-109. [CrossRef] [PubMed]

521. Lee, Y.J.; Kim, N.Y.; Suh, Y.A.; Lee, C. Involvement of ROS in Curcumin-induced Autophagic Cell Death. Korean J. Physiol. Pharmacol. 2011, 15, 1-7. [CrossRef]

522. Kim, J.Y.; Cho, T.J.; Woo, B.H.; Choi, K.U.; Lee, C.H.; Ryu, M.H.; Park, H.R. Curcumin-induced autophagy contributes to the decreased survival of oral cancer cells. Arch. Oral Biol. 2012, 57, 1018-1025. [CrossRef] [PubMed]

523. Zhuang, W.; Long, L.; Zheng, B.; Ji, W.; Yang, N.; Zhang, Q.; Liang, Z. Curcumin promotes differentiation of glioma-initiating cells by inducing autophagy. Cancer Sci. 2012, 103, 684-690. [CrossRef]

524. Wu, J.C.; Lai, C.S.; Badmaev, V.; Nagabhushanam, K.; Ho, C.T.; Pan, M.H. Tetrahydrocurcumin, a major metabolite of curcumin, induced autophagic cell death through coordinative modulation of PI3K/Akt-mTOR and MAPK signaling pathways in human leukemia HL-60 cells. Mol. Nutr. Food Res. 2011, 55, 1646-1654. [CrossRef]

525. Wang, N.; Pan, W.; Zhu, M.; Zhang, M.; Hao, X.; Liang, G.; Feng, Y. Fangchinoline induces autophagic cell death via p53/sestrin2/AMPK signalling in human hepatocellular carcinoma cells. Br. J. Pharmacol. 2011, 164, 731-742. [CrossRef] [PubMed]

526. Wong, A.S.; Che, C.M.; Leung, K.W. Recent advances in ginseng as cancer therapeutics: A functional and mechanistic overview. Nat. Prod. Rep. 2015, 32, 256-272. [CrossRef]

527. Choi, J.S.; Chun, K.S.; Kundu, J.; Kundu, J.K. Biochemical basis of cancer chemoprevention and/or chemotherapy with ginsenosides (Review). Int. J. Mol. Med. 2013, 32, 1227-1238. [CrossRef]

528. Nag, S.A.; Qin, J.J.; Wang, W.; Wang, M.H.; Wang, H.; Zhang, R. Ginsenosides as Anticancer Agents: In vitro and in vivo Activities, Structure-Activity Relationships, and Molecular Mechanisms of Action. Front. Pharmacol. 2012, 3, 25. [CrossRef]

529. Chang, Y.S.; Seo, E.K.; Gyllenhaal, C.; Block, K.I. Panax ginseng: A role in cancer therapy? Integr. Cancer Ther. 2003, 2, 13-33. [CrossRef]

530. Yun, T.K.; Lee, Y.S.; Lee, Y.H.; Kim, S.I.; Yun, H.Y. Anticarcinogenic effect of Panax ginseng C.A. Meyer and identification of active compounds. J. Korean Med. Sci. 2001, 16, S6-S18. [CrossRef]

531. Kim, S.M.; Lee, S.Y.; Cho, J.S.; Son, S.M.; Choi, S.S.; Yun, Y.P.; Yoo, H.S.; Yoon, D.Y.; Oh, K.W.; Han, S.B.; et al. Combination of ginsenoside $\mathrm{Rg} 3$ with docetaxel enhances the susceptibility of prostate cancer cells via inhibition of NF-kappaB. Eur. J. Pharmacol. 2010, 631, 1-9. [CrossRef]

532. Kim, D.G.; Jung, K.H.; Lee, D.G.; Yoon, J.H.; Choi, K.S.; Kwon, S.W.; Shen, H.M.; Morgan, M.J.; Hong, S.S.; Kim, Y.S. 20(S)Ginsenoside Rg3 is a novel inhibitor of autophagy and sensitizes hepatocellular carcinoma to doxorubicin. Oncotarget 2014, 5, 4438-4451. [CrossRef]

533. Ko, H.; Kim, Y.J.; Park, J.S.; Park, J.H.; Yang, H.O. Autophagy inhibition enhances apoptosis induced by ginsenoside Rk1 in hepatocellular carcinoma cells. Biosci. Biotechnol. Biochem. 2009, 73, 2183-2189. [CrossRef]

534. Kim, A.D.; Kang, K.A.; Kim, H.S.; Kim, D.H.; Choi, Y.H.; Lee, S.J.; Kim, H.S.; Hyun, J.W. A ginseng metabolite, compound K, induces autophagy and apoptosis via generation of reactive oxygen species and activation of JNK in human colon cancer cells. Cell Death Dis. 2013, 4, e750. [CrossRef] [PubMed]

535. Fahey, J.W.; Talalay, P. Antioxidant functions of sulforaphane: A potent inducer of Phase II detoxication enzymes. Food Chem. Toxicol. 1999, 37, 973-979. [CrossRef]

536. Herman-Antosiewicz, A.; Johnson, D.E.; Singh, S.V. Sulforaphane causes autophagy to inhibit release of cytochrome C and apoptosis in human prostate cancer cells. Cancer Res. 2006, 66, 5828-5835. [CrossRef] [PubMed] 
537. Huang, M.; Lu, J.J.; Huang, M.Q.; Bao, J.L.; Chen, X.P.; Wang, Y.T. Terpenoids: Natural products for cancer therapy. Expert Opin. Investig. Drugs 2012, 21, 1801-1818. [CrossRef]

538. Ghantous, A.; Gali-Muhtasib, H.; Vuorela, H.; Saliba, N.A.; Darwiche, N. What made sesquiterpene lactones reach cancer clinical trials? Drug Discov. Today 2010, 15, 668-678. [CrossRef]

539. Merfort, I. Perspectives on sesquiterpene lactones in inflammation and cancer. Curr. Drug Targets 2011, 12, 1560-1573. [CrossRef]

540. Kreuger, M.R.; Grootjans, S.; Biavatti, M.W.; Vandenabeele, P.; D’Herde, K. Sesquiterpene lactones as drugs with multiple targets in cancer treatment: Focus on parthenolide. Anti-Cancer Drugs 2012, 23, 883-896. [CrossRef] [PubMed]

541. D'Anneo, A.; Carlisi, D.; Lauricella, M.; Puleio, R.; Martinez, R.; Di Bella, S.; Di Marco, P.; Emanuele, S.; Di Fiore, R.; Guercio, A.; et al. Parthenolide generates reactive oxygen species and autophagy in MDA-MB231 cells. A soluble parthenolide analogue inhibits tumour growth and metastasis in a xenograft model of breast cancer. Cell Death Dis. 2013, 4, e891. [CrossRef] [PubMed]

542. Sun, J.; Zhang, C.; Bao, Y.L.; Wu, Y.; Chen, Z.L.; Yu, C.L.; Huang, Y.X.; Sun, Y.; Zheng, L.H.; Wang, X.; et al. Parthenolide-induced apoptosis, autophagy and suppression of proliferation in HepG2 cells. Asian Pac. J. Cancer Prev. 2014, 15, 4897-4902. [CrossRef] [PubMed]

543. Wagner, B.; Filice, G.A.; Drekonja, D.; Greer, N.; MacDonald, R.; Rutks, I.; Butler, M.; Wilt, T.J. Antimicrobial stewardship programs in inpatient hospital settings: A systematic review. Infect. Control Hosp. Epidemiol. 2014, 35, 1209-1228. [CrossRef]

544. Chen, L.; Liu, Q.; Huang, Z.; Wu, F.; Li, Z.; Chen, X.; Lin, T. Tripchlorolide induces cell death in lung cancer cells by autophagy. Int. J. Oncol. 2012, 40, 1066-1070. [CrossRef]

545. Yang, S.; Chen, J.; Guo, Z.; Xu, X.M.; Wang, L.; Pei, X.F.; Yang, J.; Underhill, C.B.; Zhang, L. Triptolide inhibits the growth and metastasis of solid tumors. Mol. Cancer Ther. 2003, 2, 65-72.

546. Mujumdar, N.; Mackenzie, T.N.; Dudeja, V.; Chugh, R.; Antonoff, M.B.; Borja-Cacho, D.; Sangwan, V.; Dawra, R.; Vickers, S.M.; Saluja, A.K. Triptolide induces cell death in pancreatic cancer cells by apoptotic and autophagic pathways. Gastroenterology 2010, 139, 598-608. [CrossRef]

547. Fulda, S. Betulinic Acid for cancer treatment and prevention. Int. J. Mol. Sci. 2008, 9, 1096-1107. [CrossRef]

548. Gheorgheosu, D.; Duicu, O.; Dehelean, C.; Soica, C.; Muntean, D. Betulinic acid as a potent and complex antitumor phytochemical: A minireview. Anti-Cancer Agents Med. Chem. 2014, 14, 936-945. [CrossRef]

549. Gonzalez, P.; Mader, I.; Tchoghandjian, A.; Enzenmüller, S.; Cristofanon, S.; Basit, F.; Debatin, K.M.; Fulda, S. Impairment of lysosomal integrity by B10, a glycosylated derivative of betulinic acid, leads to lysosomal cell death and converts autophagy into a detrimental process. Cell Death Differ. 2012, 19, 1337-1346. [CrossRef]

550. Yang, L.J.; Chen, Y.; He, J.; Yi, S.; Wen, L.; Zhao, J.; Zhang, B.P.; Cui, G.H. Betulinic acid inhibits autophagic flux and induces apoptosis in human multiple myeloma cells in vitro. Acta Pharmacol. Sin. 2012, 33, 1542-1548. [CrossRef]

551. Potze, L.; Mullauer, F.B.; Colak, S.; Kessler, J.H.; Medema, J.P. Betulinic acid-induced mitochondria-dependent cell death is counterbalanced by an autophagic salvage response. Cell Death Dis. 2014, 5, e1169. [CrossRef]

552. Ye, Y.C.; Wang, H.J.; Xu, L.; Liu, W.W.; Liu, B.B.; Tashiro, S.; Onodera, S.; Ikejima, T. Oridonin induces apoptosis and autophagy in murine fibrosarcoma L929 cells partly via NO-ERK-p53 positive-feedback loop signaling pathway. Acta Pharmacol. Sin. 2012, 33, 1055-1061. [CrossRef]

553. Cheng, Y.; Qiu, F.; Ikejima, T. Molecular mechanisms of oridonin-induced apoptosis and autophagy in murine fibrosarcoma L929 cells. Autophagy 2009, 5, 430-431. [CrossRef]

554. Cheng, Y.; Qiu, F.; Ye, Y.C.; Guo, Z.M.; Tashiro, S.; Onodera, S.; Ikejima, T. Autophagy inhibits reactive oxygen species-mediated apoptosis via activating p38-nuclear factor-kappa B survival pathways in oridonin-treated murine fibrosarcoma L929 cells. FEBS J. 2009, 276, 1291-1306. [CrossRef] [PubMed]

555. Banjerdpongchai, R.; Khaw-On, P. Terpinen-4-ol induces autophagic and apoptotic cell death in human leukemic HL-60 cells. Asian Pac. J. Cancer Prev. APJCP 2013, 14, 7537-7542. [CrossRef] [PubMed]

556. Yang, H.; Chen, D.; Cui, Q.C.; Yuan, X.; Dou, Q.P. Celastrol, a triterpene extracted from the Chinese "Thunder of God Vine" is a potent proteasome inhibitor and suppresses human prostate cancer growth in nude mice. Cancer Res. 2006, 66, $4758-4765$. [CrossRef] [PubMed]

557. Li, H.Y.; Zhang, J.; Sun, L.L.; Li, B.H.; Gao, H.L.; Xie, T.; Zhang, N.; Ye, Z.M. Celastrol induces apoptosis and autophagy via the ROS/JNK signaling pathway in human osteosarcoma cells: An in vitro and in vivo study. Cell Death Dis. 2015, 6, e1604. [CrossRef] [PubMed]

558. Han, X.; Sun, S.; Zhao, M.; Cheng, X.; Chen, G.; Lin, S.; Guan, Y.; Yu, X. Celastrol stimulates hypoxia-inducible factor-1 activity in tumor cells by initiating the ROS/Akt/p70S6K signaling pathway and enhancing hypoxia-inducible factor- $1 \alpha$ protein synthesis. PLoS ONE 2014, 9, e112470. [CrossRef]

559. Ma, J.; Han, L.Z.; Liang, H.; Mi, C.; Shi, H.; Lee, J.J.; Jin, X. Celastrol inhibits the HIF-1 $\alpha$ pathway by inhibition of $\mathrm{mTOR} / \mathrm{p} 70 \mathrm{~S} 6 \mathrm{~K} / \mathrm{eIF} 4 \mathrm{E}$ and ERK1/2 phosphorylation in human hepatoma cells. Oncol. Rep. 2014, 32, 235-242. [CrossRef]

560. Boridy, S.; Le, P.U.; Petrecca, K.; Maysinger, D. Celastrol targets proteostasis and acts synergistically with a heat-shock protein 90 inhibitor to kill human glioblastoma cells. Cell Death Dis. 2014, 5, e1216. [CrossRef]

561. Lee, H.W.; Jang, K.S.; Choi, H.J.; Jo, A.; Cheong, J.H.; Chun, K.H. Celastrol inhibits gastric cancer growth by induction of apoptosis and autophagy. BMB Rep. 2014, 47, 697-702. [CrossRef] [PubMed]

562. Jackson, S.J.; Singletary, K.W. Sulforaphane inhibits human MCF-7 mammary cancer cell mitotic progression and tubulin polymerization. J. Nutr. 2004, 134, 2229-2236. [CrossRef] [PubMed] 
563. Azarenko, O.; Okouneva, T.; Singletary, K.W.; Jordan, M.A.; Wilson, L. Suppression of microtubule dynamic instability and turnover in MCF7 breast cancer cells by sulforaphane. Carcinogenesis 2008, 29, 2360-2368. [CrossRef]

564. Mi, L.; Xiao, Z.; Hood, B.L.; Dakshanamurthy, S.; Wang, X.; Govind, S.; Conrads, T.P.; Veenstra, T.D.; Chung, F.L. Covalent binding to tubulin by isothiocyanates. A mechanism of cell growth arrest and apoptosis. J. Biol. Chem. 2008, 283, 22136-22146. [CrossRef] [PubMed]

565. Parnaud, G.; Li, P.; Cassar, G.; Rouimi, P.; Tulliez, J.; Combaret, L.; Gamet-Payrastre, L. Mechanism of sulforaphane-induced cell cycle arrest and apoptosis in human colon cancer cells. Nutr. Cancer 2004, 48, 198-206. [CrossRef] [PubMed]

566. Pawlik, A.; Wiczk, A.; Kaczyńska, A.; Antosiewicz, J.; Herman-Antosiewicz, A. Sulforaphane inhibits growth of phenotypically different breast cancer cells. Eur. J. Nutr. 2013, 52, 1949-1958. [CrossRef] [PubMed]

567. Dyshlovoy, S.A.; Hauschild, J.; Amann, K.; Tabakmakher, K.M.; Venz, S.; Walther, R.; Guzii, A.G.; Makarieva, T.N.; Shubina, L.K.; Fedorov, S.N.; et al. Marine alkaloid Monanchocidin a overcomes drug resistance by induction of autophagy and lysosomal membrane permeabilization. Oncotarget 2015, 6, 17328-17341. [CrossRef] [PubMed]

568. Hu, T.; Wang, L.; Zhang, L.; Lu, L.; Shen, J.; Chan, R.L.; Li, M.; Wu, W.K.; To, K.K.; Cho, C.H. Sensitivity of apoptosis-resistant colon cancer cells to tanshinones is mediated by autophagic cell death and p53-independent cytotoxicity. Phytomedicine 2015, 22, 536-544. [CrossRef] [PubMed]

569. Kim, Y.J.; Kang, K.S.; Choi, K.C.; Ko, H. Cardamonin induces autophagy and an antiproliferative effect through JNK activation in human colorectal carcinoma HCT116 cells. Bioorg. Med. Chem. Lett. 2015, 25, 2559-2564. [CrossRef] [PubMed]

570. Armstrong, J.L.; Hill, D.S.; McKee, C.S.; Hernandez-Tiedra, S.; Lorente, M.; Lopez-Valero, I.; Eleni Anagnostou, M.; Babatunde, F.; Corazzari, M.; Redfern, C.P.F.; et al. Exploiting cannabinoid-induced cytotoxic autophagy to drive melanoma cell death. J. Investig. Dermatol. 2015, 135, 1629-1637. [CrossRef] [PubMed]

571. Trzoss, L.; Fukuda, T.; Costa-Lotufo, L.V.; Jimenez, P.; La Clair, J.J.; Fenical, W. Seriniquinone, a selective anticancer agent, induces cell death by autophagocytosis, targeting the cancer-protective protein dermcidin. Proc. Natl. Acad. Sci. USA 2014, 111, 14687-14692. [CrossRef]

572. Xu, D.; Lao, Y.; Xu, N.; Hu, H.; Fu, W.; Tan, H.; Gu, Y.; Song, Z.; Cao, P.; Xu, H. Identification and characterization of anticancer compounds targeting apoptosis and autophagy from Chinese native Garcinia species. Planta Med. 2015, 81, 79-89. [CrossRef]

573. Feng, C.; Zhou, L.Y.; Yu, T.; Xu, G.; Tian, H.L.; Xu, J.J.; Xu, H.X.; Luo, K.Q. A new anticancer compound, oblongifolin C, inhibits tumor growth and promotes apoptosis in HeLa cells through Bax activation. Int. J. Cancer 2012, 131, 1445-1454. [CrossRef]

574. Lao, Y.; Wan, G.; Liu, Z.; Wang, X.; Ruan, P.; Xu, W.; Xu, D.; Xie, W.; Zhang, Y.; Xu, H.; et al. The natural compound oblongifolin C inhibits autophagic flux and enhances antitumor efficacy of nutrient deprivation. Autophagy 2014, 10, 736-749. [CrossRef]

575. Ouyang, L.; Chen, Y.; Wang, X.Y.; Lu, R.F.; Zhang, S.Y.; Tian, M.; Xie, T.; Liu, B.; He, G. Polygonatum odoratum lectin induces apoptosis and autophagy via targeting EGFR-mediated Ras-Raf-MEK-ERK pathway in human MCF-7 breast cancer cells. Phytomedicine 2014, 21, 1658-1665. [CrossRef]

576. Li, C.; Chen, J.; Lu, B.; Shi, Z.; Wang, H.; Zhang, B.; Zhao, K.; Qi, W.; Bao, J.; Wang, Y. Molecular switch role of Akt in Polygonatum odoratum lectin-induced apoptosis and autophagy in human non-small cell lung cancer A549 cells. PLoS ONE 2014, 9, e101526. [CrossRef]

577. Wang, S.Y.; Yu, Q.J.; Bao, J.K.; Liu, B. Polygonatum cyrtonema lectin, a potential antineoplastic drug targeting programmed cell death pathways. Biochem. Biophys. Res. Commun. 2011, 406, 497-500. [CrossRef]

578. Carvalho, F.C.; Soares, S.G.; Tamarozzi, M.B.; Rego, E.M.; Roque-Barreira, M.C. The recognition of N-glycans by the lectin ArtinM mediates cell death of a human myeloid leukemia cell line. PLoS ONE 2011, 6, e27892. [CrossRef]

579. Zhang, C.Z.; Fang, E.F.; Zhang, H.T.; Liu, L.L.; Yun, J.P. Momordica charantia lectin exhibits antitumor activity towards hepatocellular carcinoma. Investig. New Drugs 2015, 33, 1-11. [CrossRef]

580. Pratt, J.; Annabi, B. Induction of autophagy biomarker BNIP3 requires a JAK2/STAT3 and MT1-MMP signaling interplay in Concanavalin-A-activated U87 glioblastoma cells. Cell. Signal. 2014, 26, 917-924. [CrossRef]

581. Chen, M.C.; Lee, N.H.; Hsu, H.H.; Ho, T.J.; Tu, C.C.; Hsieh, D.J.; Lin, Y.M.; Chen, L.M.; Kuo, W.W.; Huang, C.Y. Thymoquinone induces caspase-independent, autophagic cell death in CPT-11-resistant lovo colon cancer via mitochondrial dysfunction and activation of JNK and p38. J. Agric. Food Chem. 2015, 63, 1540-1546. [CrossRef] [PubMed]

582. Hahm, E.R.; Sakao, K.; Singh, S.V. Honokiol activates reactive oxygen species-mediated cytoprotective autophagy in human prostate cancer cells. Prostate 2014, 74, 1209-1221. [CrossRef] [PubMed]

583. Zhang, L.; Wang, H.; Zhu, J.; Xu, J.; Ding, K. Mollugin induces tumor cell apoptosis and autophagy via the PI3K/AKT/mTOR/ p70S6K and ERK signaling pathways. Biochem. Biophys. Res. Commun. 2014, 450, 247-254. [CrossRef] [PubMed]

584. Xu, M.Y.; Lee, S.Y.; Kang, S.S.; Kim, Y.S. Antitumor activity of jujuboside B and the underlying mechanism via induction of apoptosis and autophagy. J. Nat. Prod. 2014, 77, 370-376. [CrossRef]

585. Kaewpiboon, C.; Surapinit, S.; Malilas, W.; Moon, J.; Phuwapraisirisan, P.; Tip-Pyang, S.; Johnston, R.N.; Koh, S.S.; Assavalapsakul, W.; Chung, Y.H. Feroniellin A-induced autophagy causes apoptosis in multidrug-resistant human A549 lung cancer cells. Int. J. Oncol. 2014, 44, 1233-1242. [CrossRef] [PubMed]

586. Jiang, Y.P.; Guo, H.; Wang, X.B. Nobiletin (NOB) suppresses autophagic degradation via over-expressing AKT pathway and enhances apoptosis in multidrug-resistant SKOV3/TAX ovarian cancer cells. Biomed. Pharmacother. 2018, 103, 29-37. [CrossRef] 
587. Zhang, H.; Ge, C.; Lin, C.; Yi, L.; Ran, J.; Shi, X.; Tang, C.; Wu, Y.; Nian, W. Combination of icotinib and wogonin induces apoptosis and autophagy to overcome acquired resistance in lung cancer harbouring EGFR T790M mutation. Int. J. Clin. Exp. Med. 2017, 10, 7553-7562.

588. Liu, Y.; Xu, Y.; Ji, W.; Li, X.; Sun, B.; Gao, Q.; Su, C. Anti-tumor activities of matrine and oxymatrine: Literature review. Tumour Biol. 2014, 35, 5111-5119. [CrossRef]

589. Mariño, G.; Niso-Santano, M.; Baehrecke, E.H.; Kroemer, G. Self-consumption: The interplay of autophagy and apoptosis. Nat. Rev. Mol. Cell Biol. 2014, 15, 81-94. [CrossRef]

590. Rambold, A.S.; Cohen, S.; Lippincott-Schwartz, J. Fatty acid trafficking in starved cells: Regulation by lipid droplet lipolysis, autophagy, and mitochondrial fusion dynamics. Dev. Cell 2015, 32, 678-692. [CrossRef]

591. Estrela, J.M.; Ortega, A.; Obrador, E. Glutathione in cancer biology and therapy. Crit. Rev. Clin. Lab. Sci. 2006, 43, 143-181. [CrossRef]

592. El-Serag, H.B.; Rudolph, K.L. Hepatocellular carcinoma: Epidemiology and molecular carcinogenesis. Gastroenterology 2007, 132, 2557-2576. [CrossRef]

593. D'Arcy, M.S. Cell death: A review of the major forms of apoptosis, necrosis and autophagy. Cell Biol. Int. 2019, 43, 582-592. [CrossRef]

594. Balunas, M.J.; Kinghorn, A.D. Drug discovery from medicinal plants. Life Sci. 2005, 78, 431-441. [CrossRef]

595. Kinghorn, A.D.; Carcache de Blanco, E.J.; Chai, H.B.; Orjala, J.; Farnsworth, N.R.; Soejarto, D.D.; Oberlies, N.H.; Wani, M.C.; Kroll, D.J.; Pearce, C.J.; et al. Discovery of anticancer agents of diverse natural origin. Pure Appl. Chem. 2009, 81, 1051-1063. [CrossRef] [PubMed]

596. Tu, Y. Artemisinin-A Gift from Traditional Chinese Medicine to the World (Nobel Lecture). Angew. Chem. Int. Ed. 2016, 55, 10210-10226. [CrossRef] [PubMed]

597. Jeong, D.; Song, H.; Lim, S.; Lee, S.; Lim, J.; Nam, D.; Joo, K.; Jeong, B.; Jeon, S.; Choi, H. Repurposing the anti-malarial drug artesunate as a novel therapeutic agent for metastatic renal cell carcinoma due to its attenuation of tumor growth, metastasis, and angiogenesis. Oncotarget 2015, 6, 33046-33064. [CrossRef] [PubMed]

598. Li, D.; Zhang, J.; Zhao, X. Mechanisms and molecular targets of artemisinin in cancer treatment. Cancer Investig. 2021, 39, 675-684. [CrossRef]

599. Xu, C.; Zhang, H.; Mu, L.; Yang, X. Artemisinins as anticancer drugs: Novel therapeutic approaches, molecular mechanisms, and clinical trials. Front. Pharmacol. 2020, 11, 1608. [CrossRef]

600. Zhou, X.; Sun, W.-J.; Wang, W.-M.; Chen, K.; Zheng, J.-H.; Lu, M.-D.; Li, P.-H.; Zheng, Z.-Q. Artesunate inhibits the growth of gastric cancer cells through the mechanism of promoting oncosis both in vitro and in vivo. Anti-Cancer Drugs 2013, 24, 920-927. [CrossRef]

601. Maltese, W.A.; Overmeyer, J.H. Methuosis: Nonapoptotic cell death associated with vacuolization of macropinosome and endosome compartments. Am. J. Pathol. 2014, 184, 1630-1642. [CrossRef]

602. Overmeyer, J.H.; Kaul, A.; Johnson, E.E.; Maltese, W.A. Active ras triggers death in glioblastoma cells through hyperstimulation of macropinocytosis. Mol. Cancer Res. 2008, 6, 965-977. [CrossRef]

603. Sun, L.; Li, B.; Su, X.; Chen, G.; Li, Y.; Yu, L.; Li, L.; Wei, W. An Ursolic Acid Derived Small Molecule Triggers Cancer Cell Death through Hyperstimulation of Macropinocytosis. J. Med. Chem. 2017, 60, 6638-6648. [CrossRef] [PubMed]

604. Song, S.; Zhang, Y.; Ding, T.; Ji, N.; Zhao, H. The Dual Role of Macropinocytosis in Cancers: Promoting Growth and Inducing Methuosis to Participate in Anticancer Therapies as Targets. Front. Oncol. 2020, 10, 570108. [CrossRef]

605. Bang, F.B. History of tissue culture at Johns Hopkins. Bull. Hist. Med. 1977, 51, 516-537. [PubMed]

606. Bar-Sagi, D.; Feramisco, J.R. Induction of membrane ruffling and fluid-phase pinocytosis in quiescent fibroblasts by ras proteins. Science 1986, 233, 1061-1068. [CrossRef] [PubMed]

607. Ridley, A.J.; Paterson, H.F.; Johnston, C.L.; Diekmann, D.; Hall, A. The small GTP-binding protein rac regulates growth factorinduced membrane ruffling. Cell 1992, 70, 401-410. [CrossRef]

608. Swanson, J.A.; Watts, C. Macropinocytosis. Trends Cell Biol. 1995, 5, 424-428. [CrossRef]

609. Chi, S.; Kitanaka, C.; Noguchi, K.; Mochizuki, T.; Nagashima, Y.; Shirouzu, M.; Fujita, H.; Yoshida, M.; Chen, W.; Asai, A.; et al. Oncogenic Ras triggers cell suicide through the activation of a caspase-independent cell death program in human cancer cells. Oncogene 1999, 18, 2281-2290. [CrossRef]

610. Zhang, D.; Tang, B.; Xie, X.; Xiao, Y.F.; Yang, S.M.; Zhang, J.W. The interplay between DNA repair and autophagy in cancer therapy. Cancer Biol. Ther. 2015, 16, 1005-1013. [CrossRef]

611. Klappan, A.K.; Hones, S.; Mylonas, I.; Brüning, A. Proteasome inhibition by quercetin triggers macroautophagy and blocks mTOR activity. Histochem. Cell Biol. 2012, 137, 25-36. [CrossRef] [PubMed]

612. Delmas, D.; Solary, E.; Latruffe, N. Resveratrol, a phytochemical inducer of multiple cell death pathways: Apoptosis, autophagy and mitotic catastrophe. Curr. Med. Chem. 2011, 18, 1100-1121. [CrossRef]

613. Arafiles, J.V.V.; Hirose, H.; Akishiba, M.; Tsuji, S.; Imanishi, M.; Futaki, S. Stimulating Macropinocytosis for Intracellular Nucleic Acid and Protein Delivery: A Combined Strategy with Membrane-Lytic Peptides To Facilitate Endosomal Escape. Bioconjug. Chem. 2020, 31, 547-553. [CrossRef] [PubMed] 
614. Commisso, C.; Davidson, S.M.; Soydaner-Azeloglu, R.G.; Parker, S.J.; Kamphorst, J.J.; Hackett, S.; Grabocka, E.; Nofal, M.; Drebin, J.A.; Thompson, C.B.; et al. Macropinocytosis of protein is an amino acid supply route in Ras-transformed cells. Nature 2013, 497, 633-637. [CrossRef] [PubMed]

615. Qian, Y.; Wang, X.; Liu, Y.; Li, Y.; Colvin, R.A.; Tong, L.; Wu, S.; Chen, X. Extracellular ATP is internalized by macropinocytosis and induces intracellular ATP increase and drug resistance in cancer cells. Cancer Lett. 2014, 351, 242-251. [CrossRef] [PubMed]

616. Palm, W.; Park, Y.; Wright, K.; Pavlova, N.N.; Tuveson, D.A.; Thompson, C.B. The Utilization of Extracellular Proteins as Nutrients Is Suppressed by mTORC1. Cell 2015, 162, 259-270. [CrossRef]

617. Tkach, M.; Théry, C. Communication by Extracellular Vesicles: Where We Are and Where We Need to Go. Cell 2016, 164, 1226-1232. [CrossRef]

618. Kim, S.M.; Nguyen, T.T.; Ravi, A.; Kubiniok, P.; Finicle, B.T.; Jayashankar, V.; Malacrida, L.; Hou, J.; Robertson, J.; Gao, D.; et al. PTEN Deficiency and AMPK Activation Promote Nutrient Scavenging and Anabolism in Prostate Cancer Cells. Cancer Discov. 2018, 8, 866-883. [CrossRef]

619. Ramirez, C.; Hauser, A.D.; Vucic, E.A.; Bar-Sagi, D. Plasma membrane V-ATPase controls oncogenic RAS-induced macropinocytosis. Nature 2019, 576, 477-481. [CrossRef]

620. Yao, W.; Rose, J.L.; Wang, W.; Seth, S.; Jiang, H.; Taguchi, A.; Liu, J.; Yan, L.; Kapoor, A.; Hou, P.; et al. Syndecan 1 is a critical mediator of macropinocytosis in pancreatic cancer. Nature 2019, 568, 410-414. [CrossRef]

621. Srivastava, R.K.; Li, C.; Khan, J.; Banerjee, N.S.; Chow, L.T.; Athar, M. Combined mTORC1/mTORC2 inhibition blocks growth and induces catastrophic macropinocytosis in cancer cells. Proc. Natl. Acad. Sci. USA 2019, 116, 24583-24592. [CrossRef]

622. Lee, S.W.; Zhang, Y.; Jung, M.; Cruz, N.; Alas, B.; Commisso, C. EGFR-Pak Signaling Selectively Regulates Glutamine DeprivationInduced Macropinocytosis. Dev. Cell 2019, 50, 381-392.e385. [CrossRef]

623. Li, Z.; Mbah, N.E.; Overmeyer, J.H.; Sarver, J.G.; George, S.; Trabbic, C.J.; Erhardt, P.W.; Maltese, W.A. The JNK signaling pathway plays a key role in methuosis (non-apoptotic cell death) induced by MOMIPP in glioblastoma. BMC Cancer 2019, 19, 77. [CrossRef] [PubMed]

624. Freeman, S.A.; Uderhardt, S.; Saric, A.; Collins, R.F.; Buckley, C.M.; Mylvaganam, S.; Boroumand, P.; Plumb, J.; Germain, R.N.; Ren, D.; et al. Lipid-gated monovalent ion fluxes regulate endocytic traffic and support immune surveillance. Science 2020, 367, 301-305. [CrossRef] [PubMed]

625. King, J.S.; Smythe, E. Water loss regulates cell and vesicle volume. Science 2020, 367, 246-247. [CrossRef] [PubMed]

626. Panda, P.K.; Mukhopadhyay, S.; Das, D.N.; Sinha, N.; Naik, P.P.; Bhutia, S.K. Mechanism of autophagic regulation in carcinogenesis and cancer therapeutics. Proc. Semin. Cell Dev. Biol. 2015, 39, 43-55. [CrossRef]

627. Reyjal, J.; Cormier, K.; Turcotte, S. Autophagy and cell death to target cancer cells: Exploiting synthetic lethality as cancer therapies. Adv. Exp. Med. Biol. 2014, 772, 167-188. [CrossRef]

628. Fulda, S. Therapeutic exploitation of necroptosis for cancer therapy. Semin. Cell Dev. Biol. 2014, 35, 51-56. [CrossRef] [PubMed]

629. Cho, Y.S.; Park, S.Y. Harnessing of Programmed Necrosis for Fighting against Cancers. Biomol. Ther. 2014, 22, 167-175. [CrossRef] [PubMed]

630. Belaid, A.; Ndiaye, P.D.; Filippakis, H.; Roux, J.; Röttinger, É; Graba, Y.; Brest, P.; Hofman, P.; Mograbi, B. Autophagy: Moving Benchside Promises to Patient Bedsides. Curr. Cancer Drug Targets 2015, 15, 684-702. [CrossRef] [PubMed]

631. Tang, J.; Di, J.; Cao, H.; Bai, J.; Zheng, J. p53-mediated autophagic regulation: A prospective strategy for cancer therapy. Cancer Lett. 2015, 363, 101-107. [CrossRef] [PubMed]

632. Li, S.; Wang, L.; Hu, Y.; Sheng, R. Autophagy regulators as potential cancer therapeutic agents: A review. Curr. Top. Med. Chem. 2015, 15, 720-744. [CrossRef] [PubMed]

633. Guamán-Ortiz, L.M.; Orellana, M.I.; Ratovitski, E.A. Natural Compounds As Modulators of Non-apoptotic Cell Death in Cancer Cells. Curr. Genom. 2017, 18, 132-155. [CrossRef]

634. Ozpolat, B.; Akar, U.; Mehta, K.; Lopez-Berestein, G. PKC delta and tissue transglutaminase are novel inhibitors of autophagy in pancreatic cancer cells. Autophagy 2007, 3, 480-483. [CrossRef]

635. Christian, F.; Anthony, D.F.; Vadrevu, S.; Riddell, T.; Day, J.P.; McLeod, R.; Adams, D.R.; Baillie, G.S.; Houslay, M.D. p62 (SQSTM1) and cyclic AMP phosphodiesterase-4A4 (PDE4A4) locate to a novel, reversible protein aggregate with links to autophagy and proteasome degradation pathways. Cell. Signal. 2010, 22, 1576-1596. [CrossRef]

636. Ali, A.B.; Nin, D.S.; Tam, J.; Khan, M. Role of chaperone mediated autophagy (CMA) in the degradation of misfolded N-CoR protein in non-small cell lung cancer (NSCLC) cells. PLoS ONE 2011, 6, e25268. [CrossRef]

637. Lao, F.; Shang, Y.; Liu, D. Autophagy pathway of raji cell death induced by resveratrol. Chin. J. Biol. 2009, 22, 654-658.

638. Lao, C.D.; Demierre, M.F.; Sondak, V.K. Targeting events in melanoma carcinogenesis for the prevention of melanoma. Expert Rev. Anticancer. Ther. 2006, 6, 1559-1568. [CrossRef]

639. Scarlatti, F.; Maffei, R.; Beau, I.; Ghidoni, R.; Codogno, P. Non-canonical autophagy: An exception or an underestimated form of autophagy? Autophagy 2008, 4, 1083-1085. [CrossRef] [PubMed]

640. Puissant, A.; Auberger, P. AMPK- and p62/SQSTM1-dependent autophagy mediate resveratrol-induced cell death in chronic myelogenous leukemia. Autophagy 2010, 6, 655-657. [CrossRef]

641. Zhang, J.; Ma, K.; Qi, T.; Wei, X.; Zhang, Q.; Li, G.; Chiu, J.F. P62 regulates resveratrol-mediated Fas/Cav-1 complex formation and transition from autophagy to apoptosis. Oncotarget 2015, 6, 789-801. [CrossRef] [PubMed] 
642. Dalby, K.N.; Tekedereli, I.; Lopez-Berestein, G.; Ozpolat, B. Targeting the prodeath and prosurvival functions of autophagy as novel therapeutic strategies in cancer. Autophagy 2010, 6, 322-329. [CrossRef] [PubMed]

643. Morselli, E.; Mariño, G.; Bennetzen, M.V.; Eisenberg, T.; Megalou, E.; Schroeder, S.; Cabrera, S.; Bénit, P.; Rustin, P.; Criollo, A.; et al. Spermidine and resveratrol induce autophagy by distinct pathways converging on the acetylproteome. J. Cell Biol. 2011, 192, 615-629. [CrossRef]

644. Filippi-Chiela, E.C.; Villodre, E.S.; Zamin, L.L.; Lenz, G. Autophagy interplay with apoptosis and cell cycle regulation in the growth inhibiting effect of resveratrol in glioma cells. PLoS ONE 2011, 6, e20849. [CrossRef] [PubMed]

645. Mauthe, M.; Jacob, A.; Freiberger, S.; Hentschel, K.; Stierhof, Y.D.; Codogno, P.; Proikas-Cezanne, T. Resveratrol-mediated autophagy requires WIPI-1-regulated LC3 lipidation in the absence of induced phagophore formation. Autophagy 2011, 7, 1448-1461. [CrossRef]

646. Miki, H.; Uehara, N.; Kimura, A.; Sasaki, T.; Yuri, T.; Yoshizawa, K.; Tsubura, A. Resveratrol induces apoptosis via ROS-triggered autophagy in human colon cancer cells. Int. J. Oncol. 2012, 40, 1020-1028. [CrossRef]

647. Back, J.H.; Zhu, Y.; Calabro, A.; Queenan, C.; Kim, A.S.; Arbesman, J.; Kim, A.L. Resveratrol-mediated downregulation of Rictor attenuates autophagic process and suppresses UV-induced skin carcinogenesis. Photochem. Photobiol. 2012, 88, 1165-1172. [CrossRef]

648. Lee, K.H.; Lo, H.L.; Tang, W.C.; Hsiao, H.H.; Yang, P.M. A gene expression signature-based approach reveals the mechanisms of action of the Chinese herbal medicine berberine. Sci. Rep. 2014, 4, 6394. [CrossRef]

649. Lee, M.-H.; Kundu, J.K.; Keum, Y.-S.; Cho, Y.-Y.; Surh, Y.-J.; Choi, B.Y. Resveratrol inhibits IL-6-induced transcriptional activity of AR and STAT3 in human prostate cancer LNCaP-FGC cells. Biomol. Ther. 2014, 22, 426. [CrossRef]

650. Luo, H.; Vong, C.T.; Chen, H.; Gao, Y.; Lyu, P.; Qiu, L.; Zhao, M.; Liu, Q.; Cheng, Z.; Zou, J. Naturally occurring anti-cancer compounds: Shining from Chinese herbal medicine. Chin. Med. 2019, 14, 1-58.

651. Thongrakard, V.; Titone, R.; Follo, C.; Morani, F.; Suksamrarn, A.; Tencomnao, T.; Isidoro, C. Turmeric toxicity in A431 epidermoid cancer cells associates with autophagy degradation of anti-apoptotic and anti-autophagic p53 mutant. Phytother. Res. 2014, 28, 1761-1769. [CrossRef] [PubMed]

652. Li, B.; Takeda, T.; Tsuiji, K.; Wong, T.F.; Tadakawa, M.; Kondo, A.; Nagase, S.; Yaegashi, N. Curcumin induces cross-regulation between autophagy and apoptosis in uterine leiomyosarcoma cells. Int. J. Gynecol. Cancer 2013, 23, 803-808. [CrossRef] [PubMed]

653. Yamauchi, Y.; Izumi, Y.; Asakura, K.; Hayashi, Y.; Nomori, H. Curcumin induces autophagy in ACC-MESO-1 cells. Phytother. Res. 2012, 26, 1779-1783. [CrossRef] [PubMed]

654. Han, J.; Pan, X.Y.; Xu, Y.; Xiao, Y.; An, Y.; Tie, L.; Pan, Y.; Li, X.J. Curcumin induces autophagy to protect vascular endothelial cell survival from oxidative stress damage. Autophagy 2012, 8, 812-825. [CrossRef]

655. Bisht, K.; Wagner, K.H.; Bulmer, A.C. Curcumin, resveratrol and flavonoids as anti-inflammatory, cyto- and DNA-protective dietary compounds. Toxicology 2010, 278, 88-100. [CrossRef]

656. Guo, J.; Huang, X.; Wang, H.; Yang, H. Celastrol Induces Autophagy by Targeting AR/miR-101 in Prostate Cancer Cells. PLoS ONE 2015, 10, e0140745. [CrossRef]

657. Aggarwal, B.B.; Kumar, A.; Bharti, A.C. Anticancer potential of curcumin: Preclinical and clinical studies. Anticancer. Res. 2003, 23, 363-398.

658. De Clercq, E. Current lead natural products for the chemotherapy of human immunodeficiency virus (HIV) infection. Med. Res. Rev. 2000, 20, 323-349. [CrossRef]

659. Larocque, K.; Ovadje, P.; Djurdjevic, S.; Mehdi, M.; Green, J.; Pandey, S. Novel analogue of colchicine induces selective pro-death autophagy and necrosis in human cancer cells. PLoS ONE 2014, 9, e87064. [CrossRef] [PubMed]

660. Amagase, H. Clarifying the real bioactive constituents of garlic. J. Nutr. 2006, 136, 716s-725s. [CrossRef] [PubMed]

661. Altonsy, M.O.; Andrews, S.C. Diallyl disulphide, a beneficial component of garlic oil, causes a redistribution of cell-cycle growth phases, induces apoptosis, and enhances butyrate-induced apoptosis in colorectal adenocarcinoma cells (HT-29). Nutr. Cancer 2011, 63, 1104-1113. [CrossRef]

662. Chu, Y.L.; Ho, C.T.; Chung, J.G.; Rajasekaran, R.; Sheen, L.Y. Allicin induces p53-mediated autophagy in Hep G2 human liver cancer cells. J. Agric. Food Chem. 2012, 60, 8363-8371. [CrossRef]

663. Chu, Y.L.; Ho, C.T.; Chung, J.G.; Raghu, R.; Lo, Y.C.; Sheen, L.Y. Allicin induces anti-human liver cancer cells through the p53 gene modulating apoptosis and autophagy. J. Agric. Food Chem. 2013, 61, 9839-9848. [CrossRef]

664. Lyu, Q.; Tou, F.; Su, H.; Wu, X.; Chen, X.; Zheng, Z. The natural product peiminine represses colorectal carcinoma tumor growth by inducing autophagic cell death. Biochem. Biophys. Res. Commun. 2015, 462, 38-45. [CrossRef]

665. Reya, T.; Morrison, S.J.; Clarke, M.F.; Weissman, I.L. Stem cells, cancer, and cancer stem cells. Nature 2001, 414, 105-111. [CrossRef]

666. Dennis, T.; Fanous, M.; Mousa, S. Natural products for chemopreventive and adjunctive therapy in oncologic disease. Nutr. Cancer 2009, 61, 587-597. [CrossRef]

667. Chai, S.; To, K.K.; Lin, G. Circumvention of multi-drug resistance of cancer cells by Chinese herbal medicines. Chin. Med. 2010, 5, 26. [CrossRef]

668. Yu-Jen, C. Potential role of tetrandrine in cancer therapy. Acta Pharmacol. Sin. 2002, 23, 1102-1106.

669. Talib, W.H.; Alsayed, A.R.; Abuawad, A.; Daoud, S.; Mahmod, A.I. Melatonin in Cancer Treatment: Current Knowledge and Future Opportunities. Molecules 2021, 26, 2506. [CrossRef] 
670. Ma, Z.; Xu, L.; Liu, D.; Zhang, X.; Di, S.; Li, W.; Zhang, J.; Reiter, R.J.; Han, J.; Li, X.; et al. Utilizing Melatonin to Alleviate Side Effects of Chemotherapy: A Potentially Good Partner for Treating Cancer with Ageing. Oxid. Med. Cell. Longev. 2020, 2020, 6841581. [CrossRef]

671. Qazi, M.A.; Vora, P.; Venugopal, C.; Sidhu, S.S.; Moffat, J.; Swanton, C.; Singh, S.K. Intratumoral heterogeneity: Pathways to treatment resistance and relapse in human glioblastoma. Ann. Oncol. 2017, 28, 1448-1456. [CrossRef] [PubMed]

672. Maitra, S.; Bhattacharya, D.; Das, S.; Bhattacharya, S. Melatonin and its anti-glioma functions: A comprehensive review. Rev. Neurosci. 2019, 30, 527-541. [CrossRef] [PubMed]

673. Neamati, F.; Asemi, Z. The effects of melatonin on signaling pathways and molecules involved in glioma. Fundam. Clin. Pharmacol. 2020, 34, 192-199. [CrossRef] [PubMed]

674. Lissoni, P.; Giani, L.; Zerbini, S.; Trabattoni, P.; Rovelli, F. Biotherapy with the pineal immunomodulating hormone melatonin versus melatonin plus aloe vera in untreatable advanced solid neoplasms. Nat. Immun. 1998, 16, 27-33. [CrossRef]

675. McConnell, D.D.; McGreevy, J.W.; Williams, M.N.; Litofsky, N.S. Do Anti-Oxidants Vitamin D(3,) Melatonin, and Alpha-Lipoic Acid Have Synergistic Effects with Temozolomide on Cultured Glioblastoma Cells? Medicines 2018, 5, 58. [CrossRef]

676. Zhou, X.; Seto, S.W.; Chang, D.; Kiat, H.; Razmovski-Naumovski, V.; Chan, K.; Bensoussan, A. Synergistic Effects of Chinese Herbal Medicine: A Comprehensive Review of Methodology and Current Research. Front. Pharmacol. 2016, 7, 201. [CrossRef]

677. Soignet, S.L.; Frankel, S.R.; Douer, D.; Tallman, M.S.; Kantarjian, H.; Calleja, E.; Stone, R.M.; Kalaycio, M.; Scheinberg, D.A.; Steinherz, P.; et al. United States Multicenter Study of Arsenic Trioxide in Relapsed Acute Promyelocytic Leukemia. J. Clin. Oncol. 2001, 19, 3852-3860. [CrossRef]

678. Liu, J.; Wang, S.; Zhang, Y.; Fan, H.T.; Lin, H.S. Traditional Chinese medicine and cancer: History, present situation, and development. Thorac. Cancer 2015, 6, 561-569. [CrossRef] [PubMed]

679. Jiang, C.M.; Pang, M.R.; Gong, L.Y. Clinical observation on effect of chemotherapy combined with Chinese medicine in treating advanced tumor patients and on immunologic parameters. Zhongguo Zhong Xi Yi Jie He Za Zhi (Chin. J. Integr. Tradit. West. Med.) 2001, 21, 885-887.

680. Yan, G.Y.; Xu, Z.Y.; Deng, H.B.; Wan, Z.Y.; Zhang, L.; Zhu, J.Y. Effects of chemotherapy combined with Chinese herbal medicine Kangliu Zengxiao decoction on tumor markers of patients with advanced non-small-cell lung cancer: A randomized, controlled trial. Zhong Xi Yi Jie He Xue Bao (J. Chin. Integr. Med.) 2011, 9, 525-530. [CrossRef] [PubMed]

681. Tang, Z. Combination of traditional Chinese medicine and western medicine in the treatment of liver cancer. J. Clin. Hepatol. 2011, $27,449-450$.

682. Shen, F.; Jin, J.; Cao, F. Clinical observation of combination of Chinese medicines combined with chemoradiotherapy in treatment of patients with stage-III non-small-cell lung cancer. Med. J. West China 2014, 26, 445-449.

683. Chahar, M.K.; Sharma, N.; Dobhal, M.P.; Joshi, Y.C. Flavonoids: A versatile source of anticancer drugs. Pharmacogn. Rev. 2011, 5, 1-12. [CrossRef]

684. Chearwae, W.; Anuchapreeda, S.; Nandigama, K.; Ambudkar, S.; Limtrakul, P. Biochemical mechanism of modulation of human P-glycoprotein (ABCB1) by curcumin I, II, and III purified from Turmeric powder. Biochem. Pharmacol. 2004, 68, $2043-2052$. [CrossRef]

685. Fang, H.Y.; Chen, S.B.; Guo, D.J.; Pan, S.Y.; Yu, Z.L. Proteomic identification of differentially expressed proteins in curcumin-treated MCF-7 cells. Phytomedicine 2011, 18, 697-703. [CrossRef]

686. Ravindran, J.; Prasad, S.; Aggarwal, B.B. Curcumin and Cancer Cells: How Many Ways Can Curry Kill Tumor Cells Selectively? AAPS J. 2009, 11, 495-510. [CrossRef]

687. Jurenka, J.S. Anti-inflammatory properties of curcumin, a major constituent of Curcuma longa: A review of preclinical and clinical research. Altern. Med. Rev. 2009, 14, 141-153.

688. Shishodia, S.; Singh, T.; Chaturvedi, M.M. Modulation of transcription factors by curcumin. Adv. Exp. Med. Biol. 2007, 595, 127-148. [CrossRef] [PubMed]

689. Limtrakul, P.; Chearwae, W.; Shukla, S.; Phisalphong, C.; Ambudkar, S.V. Modulation of function of three ABC drug transporters, P-glycoprotein (ABCB1), mitoxantrone resistance protein (ABCG2) and multidrug resistance protein 1 (ABCC1) by tetrahydrocurcumin, a major metabolite of curcumin. Mol. Cell. Biochem. 2007, 296, 85-95. [CrossRef] [PubMed]

690. Chearwae, W.; Wu, C.P.; Chu, H.Y.; Lee, T.R.; Ambudkar, S.V.; Limtrakul, P. Curcuminoids purified from turmeric powder modulate the function of human multidrug resistance protein 1 (ABCC1). Cancer Chemother. Pharmacol. 2006, 57, 376-388. [CrossRef] [PubMed]

691. Chearwae, W.; Shukla, S.; Limtrakul, P.; Ambudkar, S.V. Modulation of the function of the multidrug resistance-linked ATPbinding cassette transporter ABCG2 by the cancer chemopreventive agent curcumin. Mol. Cancer Ther. 2006, 5, $1995-2006$. [CrossRef]

692. Shukla, S.; Zaher, H.; Hartz, A.; Bauer, B.; Ware, J.A.; Ambudkar, S.V. Curcumin inhibits the activity of ABCG2/BCRP1, a multidrug resistance-linked ABC drug transporter in mice. Pharm. Res. 2009, 26, 480-487. [CrossRef]

693. Sharma, R.; Gescher, A.; Steward, W. Curcumin: The story so far. Eur. J. Cancer 2005, 41, 1955-1968. [CrossRef]

694. Parsons, H.A.; Baracos, V.E.; Hong, D.S.; Abbruzzese, J.; Bruera, E.; Kurzrock, R. The effects of curcumin (diferuloylmethane) on body composition of patients with advanced pancreatic cancer. Oncotarget 2016, 7, 20293. [CrossRef] 
695. Kanai, M.; Otsuka, Y.; Otsuka, K.; Sato, M.; Nishimura, T.; Mori, Y.; Kawaguchi, M.; Hatano, E.; Kodama, Y.; Matsumoto, S. A phase I study investigating the safety and pharmacokinetics of highly bioavailable curcumin (Theracurmin ${ }^{\circledR}$ ) in cancer patients. Cancer Chemother. Pharmacol. 2013, 71, 1521-1530. [CrossRef]

696. Singh, S. From exotic spice to modern drug? Cell 2007, 130, 765-768. [CrossRef]

697. Lao, C.D.; Ruffin, M.T.T.; Normolle, D.; Heath, D.D.; Murray, S.I.; Bailey, J.M.; Boggs, M.E.; Crowell, J.; Rock, C.L.; Brenner, D.E. Dose escalation of a curcuminoid formulation. BMC Complementary Altern. Med. 2006, 6, 10. [CrossRef]

698. Anand, P.; Kunnumakkara, A.B.; Newman, R.A.; Aggarwal, B.B. Bioavailability of Curcumin: Problems and Promises. Mol. Pharm. 2007, 4, 807-818. [CrossRef]

699. Li, L.; Braiteh, F.S.; Kurzrock, R. Liposome-encapsulated curcumin: In vitro and in vivo effects on proliferation, apoptosis, signaling, and angiogenesis. Cancer 2005, 104, 1322-1331. [CrossRef]

700. Bisht, S.; Feldmann, G.; Soni, S.; Ravi, R.; Karikar, C.; Maitra, A.; Maitra, A. Polymeric nanoparticle-encapsulated curcumin ("nanocurcumin"): A novel strategy for human cancer therapy. J. Nanobiotechnol. 2007, 5, 3. [CrossRef]

701. Shaikh, J.; Ankola, D.D.; Beniwal, V.; Singh, D.; Kumar, M.N. Nanoparticle encapsulation improves oral bioavailability of curcumin by at least 9 -fold when compared to curcumin administered with piperine as absorption enhancer. Eur. J. Pharm. Sci. 2009, 37, 223-230. [CrossRef]

702. Mosley, C.A.; Liotta, D.C.; Snyder, J.P. Highly active anticancer curcumin analogues. Adv. Exp. Med. Biol. 2007, 595, 77-103. [CrossRef] [PubMed]

703. Shoba, G.; Joy, D.; Joseph, T.; Majeed, M.; Rajendran, R.; Srinivas, P.S. Influence of piperine on the pharmacokinetics of curcumin in animals and human volunteers. Planta Med. 1998, 64, 353-356. [CrossRef]

704. Greil, R.; Greil-Ressler, S.; Weiss, L.; Schönlieb, C.; Magnes, T.; Radl, B.; Bolger, G.T.; Vcelar, B.; Sordillo, P.P. A phase 1 doseescalation study on the safety, tolerability and activity of liposomal curcumin (Lipocurc ${ }^{\mathrm{TM}}$ ) in patients with locally advanced or metastatic cancer. Cancer Chemother. Pharmacol. 2018, 82, 695-706. [CrossRef] [PubMed]

705. Mahammedi, H.; Planchat, E.; Pouget, M.; Durando, X.; Curé, H.; Guy, L.; Van-Praagh, I.; Savareux, L.; Atger, M.; Bayet-Robert, $\mathrm{M}$. The new combination docetaxel, prednisone and curcumin in patients with castration-resistant prostate cancer: A pilot phase II study. Oncology 2016, 90, 69-78. [CrossRef] [PubMed]

706. Nelson, K.M.; Dahlin, J.L.; Bisson, J.; Graham, J.; Pauli, G.F.; Walters, M.A. The Essential Medicinal Chemistry of Curcumin. J. Med. Chem. 2017, 60, 1620-1637. [CrossRef]

707. Baker, M. Deceptive curcumin offers cautionary tale for chemists. Nature 2017, 541, 144-145. [CrossRef]

708. Cheng, A.L.; Hsu, C.H.; Lin, J.K.; Hsu, M.M.; Ho, Y.F.; Shen, T.S.; Ko, J.Y.; Lin, J.T.; Lin, B.R.; Ming-Shiang, W.; et al. Phase I clinical trial of curcumin, a chemopreventive agent, in patients with high-risk or pre-malignant lesions. Anticancer. Res. 2001, 21, 2895-2900. [PubMed]

709. Grant, K.L.; Schneider, C.D. Turmeric. Am. J. Health-Syst. Pharm. 2000, 57, 1121-1122. [CrossRef] [PubMed]

710. Dhillon, N.; Aggarwal, B.B.; Newman, R.A.; Wolff, R.A.; Kunnumakkara, A.B.; Abbruzzese, J.L.; Ng, C.S.; Badmaev, V.; Kurzrock, R. Phase II trial of curcumin in patients with advanced pancreatic cancer. Clin. Cancer Res. 2008, 14, 4491-4499. [CrossRef]

711. Narayana, K.R.; Reddy, M.S.; Chaluvadi, M.; Krishna, D. Bioflavonoids classification, pharmacological, biochemical effects and therapeutic potential. Indian J. Pharmacol. 2001, 33, 2-16.

712. Patil, B.S.; Jayaprakasha, G.; Murthy, K.N.C.; Vikram, A. Bioactive Compounds: Historical Perspectives, Opportunities, and Challenges. J. Agric. Food Chem. 2009, 57, 8142-8160. [CrossRef] [PubMed]

713. Crozier, A.; Jaganath, I.B.; Clifford, M.N. Dietary phenolics: Chemistry, bioavailability and effects on health. Nat. Prod. Rep. 2009, 26, 1001-1043. [CrossRef]

714. Cushman, M.; Nagarathnam, D. Cytotoxicities of some flavonoid analogues. J. Nat. Prod. 1991, 54, 1656-1660. [CrossRef]

715. Wang, H.K.; Xia, Y.; Yang, Z.Y.; Natschke, S.L.; Lee, K.H. Recent advances in the discovery and development of flavonoids and their analogues as antitumor and anti-HIV agents. Adv. Exp. Med. Biol. 1998, 439, 191-225. [CrossRef]

716. Choi, S.U.; Ryu, S.Y.; Yoon, S.K.; Jung, N.P.; Park, S.H.; Kim, K.H.; Choi, E.J.; Lee, C.O. Effects of flavonoids on the growth and cell cycle of cancer cells. Anticancer Res. 1999, 19, 5229-5233.

717. Leslie, E.M.; Mao, Q.; Oleschuk, C.J.; Deeley, R.G.; Cole, S.P. Modulation of multidrug resistance protein 1 (MRP1/ABCC1) transport and atpase activities by interaction with dietary flavonoids. Mol. Pharmacol. 2001, 59, 1171-1180. [CrossRef]

718. Zhang, S.; Yang, X.; Morris, M.E. Flavonoids are inhibitors of breast cancer resistance protein (ABCG2)-mediated transport. Mol. Pharmacol. 2004, 65, 1208-1216. [CrossRef]

719. Wu, C.P.; Calcagno, A.M.; Hladky, S.B.; Ambudkar, S.V.; Barrand, M.A. Modulatory effects of plant phenols on human multidrugresistance proteins 1, 4 and 5 (ABCC1, 4 and 5). FEBS J. 2005, 272, 4725-4740. [CrossRef]

720. Morris, M.E.; Zhang, S. Flavonoid-drug interactions: Effects of flavonoids on ABC transporters. Life Sci. 2006, 78, 2116-2130. [CrossRef]

721. Efferth, T. From ancient herb to modern drug: Artemisia annua and artemisinin for cancer therapy. Proc. Semin. Cancer Biol. 2017, 46, 65-83. [CrossRef]

722. Zhang, Y.; Xu, G.; Zhang, S.; Wang, D.; Prabha, P.S.; Zuo, Z. Antitumor Research on Artemisinin and Its Bioactive Derivatives. Nat. Prod. Bioprospect. 2018, 8, 303-319. [CrossRef] [PubMed] 
723. Tan, C.J.; Zhao, Y.; Goto, M.; Hsieh, K.Y.; Yang, X.M.; Morris-Natschke, S.L.; Liu, L.N.; Zhao, B.Y.; Lee, K.H. Alkaloids from Oxytropis ochrocephala and antiproliferative activity of sophoridine derivatives against cancer cell lines. Bioorg. Med. Chem. Lett. 2016, 26, 1495-1497. [CrossRef]

724. Ma, Y.; Gao, H.; Liu, J.; Chen, L.; Zhang, Q.; Wang, Z. Identification and determination of the chemical constituents in a herbal preparation, compound kushen injection, by HPLC and LC-DAD-MS/MS. J. Liq. Chromatogr. Relat. Technol. 2013, 37, 207-220. [CrossRef]

725. Wang, W.; You, R.L.; Qin, W.J.; Hai, L.N.; Fang, M.J.; Huang, G.H.; Kang, R.X.; Li, M.H.; Qiao, Y.F.; Li, J.W.; et al. Anti-tumor activities of active ingredients in Compound Kushen Injection. Acta Pharmacol. Sin. 2015, 36, 676-679. [CrossRef] [PubMed]

726. Zhou, D.-H.; Wang, X.; Yang, M.; Shi, X.; Huang, W.; Feng, Q. Combination of low concentration of (-)-epigallocatechin gallate (EGCG) and curcumin strongly suppresses the growth of non-small cell lung cancer in vitro and in vivo through causing cell cycle arrest. Int. J. Mol. Sci. 2013, 14, 12023-12036. [CrossRef]

727. Liu, T.; Liu, M.; Liu, H.; Ren, Y.; Zhao, Y.; Yan, H.; Wang, Q.-P.; Zhang, N.; Ding, Z.; Wang, Z. Co-encapsulation of (-)epigallocatechin-3-gallate and piceatannol/oxyresveratrol in $\beta$-lactoglobulin: Effect of ligand-protein binding on the antioxidant activity, stability, solubility and cytotoxicity. Food Funct. 2021, 16. [CrossRef]

728. Wang, W.; Chen, D.; Zhu, K. SOX2OT variant 7 contributes to the synergistic interaction between EGCG and Doxorubicin to kill osteosarcoma via autophagy and stemness inhibition. J. Exp. Clin. Cancer Res. 2018, 37, 1-16. [CrossRef]

729. Zhao, H.; Zhu, W.; Jia, L.; Sun, X.; Chen, G.; Zhao, X.; Li, X.; Meng, X.; Kong, L.; Xing, L. Phase I study of topical epigallocatechin3-gallate (EGCG) in patients with breast cancer receiving adjuvant radiotherapy. Br. J. Radiol. 2016, 89, 20150665. [CrossRef]

730. Zhao, H.; Xie, P.; Li, X.; Zhu, W.; Sun, X.; Sun, X.; Chen, X.; Xing, L.; Yu, J. A prospective phase II trial of EGCG in treatment of acute radiation-induced esophagitis for stage III lung cancer. Radiother. Oncol. 2015, 114, 351-356. [CrossRef]

731. Wang, X.; Chen, W. Gambogic acid is a novel anti-cancer agent that inhibits cell proliferation, angiogenesis and metastasis. Anti-Cancer Agents Med. Chem. 2012, 12, 994-1000. [CrossRef]

732. Liu, L.; Qi, X.-J.; Zhong, Z.-K.; Zhang, E.-N. Nanomedicine-based combination of gambogic acid and retinoic acid chlorochalcone for enhanced anticancer efficacy in osteosarcoma. Biomed. Pharmacother. 2016, 83, 79-84. [CrossRef] [PubMed]

733. Wang, H.; Zhao, Z.; Lei, S.; Li, S.; Xiang, Z.; Wang, X.; Huang, X.; Xia, G.; Huang, X. Gambogic acid induces autophagy and combines synergistically with chloroquine to suppress pancreatic cancer by increasing the accumulation of reactive oxygen species. Cancer Cell Int. 2019, 19, 1-15. [CrossRef] [PubMed]

734. Zhang, W.; Zhou, H.; Yu, Y.; Li, J.; Li, H.; Jiang, D.; Chen, Z.; Yang, D.; Xu, Z.; Yu, Z. Combination of gambogic acid with cisplatin enhances the antitumor effects on cisplatin-resistant lung cancer cells by downregulating MRP2 and LRP expression. OncoTargets Ther. 2016, 9, 3359. [CrossRef] [PubMed]

735. Xia, G.; Wang, H.; Song, Z.; Meng, Q.; Huang, X.; Huang, X. Gambogic acid sensitizes gemcitabine efficacy in pancreatic cancer by reducing the expression of ribonucleotide reductase subunit-M2 (RRM2). J. Exp. Clin. Cancer Res. 2017, 36, 1-14. [CrossRef] [PubMed]

736. Yihebali, C.; Zhan, X.-K.; Hao, Y.; Xie, G.-R.; Wang, Z.-Z.; Wei, X.; Wang, Y.-G.; Xiong, F.-X.; Hu, J.-F.; Lin, Y. An open-labeled, randomized, multicenter phase IIa study of gambogic acid injection for advanced malignant tumors. Chin. Med. J. 2013, 126, 1642-1646.

737. Goodall, M.L.; Fitzwalter, B.E.; Zahedi, S.; Wu, M.; Rodriguez, D.; Mulcahy-Levy, J.M.; Green, D.R.; Morgan, M.; Cramer, S.D.; Thorburn, A. The Autophagy Machinery Controls Cell Death Switching between Apoptosis and Necroptosis. Dev. Cell 2016, 37, 337-349. [CrossRef]

738. Chen, H.Y.; White, E. Role of autophagy in cancer prevention. Cancer Prev. Res. 2011, 4, 973-983. [CrossRef]

739. Rosenfeldt, M.T.; O’Prey, J.; Morton, J.P.; Nixon, C.; MacKay, G.; Mrowinska, A.; Au, A.; Rai, T.S.; Zheng, L.; Ridgway, R.; et al. p53 status determines the role of autophagy in pancreatic tumour development. Nature 2013, 504, 296-300. [CrossRef]

740. Huo, Y.; Cai, H.; Teplova, I.; Bowman-Colin, C.; Chen, G.; Price, S.; Barnard, N.; Ganesan, S.; Karantza, V.; White, E.; et al. Autophagy Opposes p53-Mediated Tumor Barrier to Facilitate Tumorigenesis in a Model of PALB2-Associated Hereditary Breast Cancer. Cancer Discov. 2013, 3, 894-907. [CrossRef]

741. Hu, K.; Chen, X.; Zuo, M.; Hou, L.; Sun, Z.; Li, Z.; Jiang, M.; Li, W.; Wang, F.; Su, W.; et al. Fritillaria thunbergii powder overcomes drug resistance of acute leukemia patients. China J. Tradit. Chin. Med. Pharm. 2004, 19, 68-70.

742. Wei, L.; Kaiwen, H.; Wei, S. Clinical trial of Fritillaria thunbergii Bulb powder for reversing multidrug resistance in the patients with acute leukemia. J. Beijing Univ. Tradit. Chin. Med. 2004, 27, 63-65.

743. Yongchen, Z.; Xinyi, C.; Yamei, X. Principal Clinical Study on Ligustrazine Injection to Counteract the Multi-drug Resistance of Acute Leukemia. Chin. J. Inf. Tradit. Chin. Med. 2003, 12, 10-11.

744. Deeken, J.F.; Wang, H.; Hartley, M.; Cheema, A.K.; Smaglo, B.; He, A.R.; Weiner, L.M.; Marshall, J.L.; Giaccone, G.; Liu, S.; et al. A phase I study of intravenous artesunate in patients with advanced solid tumor malignancies. Cancer Chemother. Pharmacol. 2018, 81, 587-596. [CrossRef]

745. Von Hagens, C.; Walter-Sack, I.; Goeckenjan, M.; Osburg, J.; Storch-Hagenlocher, B.; Sertel, S.; Elsässer, M.; Remppis, B.A.; Edler, L.; Munzinger, J.; et al. Prospective open uncontrolled phase I study to define a well-tolerated dose of oral artesunate as add-on therapy in patients with metastatic breast cancer (ARTIC M33/2). Breast Cancer Res. Treat. 2017, 164, 359-369. [CrossRef] [PubMed] 
746. von Hagens, C.; Walter-Sack, I.; Goeckenjan, M.; Storch-Hagenlocher, B.; Sertel, S.; Elsässer, M.; Remppis, B.A.; Munzinger, J.; Edler, L.; Efferth, T.; et al. Long-term add-on therapy (compassionate use) with oral artesunate in patients with metastatic breast cancer after participating in a phase I study (ARTIC M33/2). Phytomedicine 2019, 54, 140-148. [CrossRef] [PubMed]

747. Towers, C.G.; Thorburn, A. Therapeutic Targeting of Autophagy. EBioMedicine 2016, 14, 15-23. [CrossRef] [PubMed]

748. Markman, M. Safety issues in using complementary and alternative medicine. J. Clin. Oncol. 2002, 20 (Suppl. 18), 39S-41S.

749. Ben-Arye, E.; Samuels, N.; Goldstein, L.H.; Mutafoglu, K.; Omran, S.; Schiff, E.; Charalambous, H.; Dweikat, T.; Ghrayeb, I.; Bar-Sela, G.; et al. Potential risks associated with traditional herbal medicine use in cancer care: A study of Middle Eastern oncology health care professionals. Cancer 2016, 122, 598-610. [CrossRef] [PubMed]

750. Jatoi, A.; Ellison, N.; Burch, P.A.; Sloan, J.A.; Dakhil, S.R.; Novotny, P.; Tan, W.; Fitch, T.R.; Rowland, K.M.; Young, C.Y.; et al. A phase II trial of green tea in the treatment of patients with androgen independent metastatic prostate carcinoma. Cancer 2003, 97, 1442-1446. [CrossRef]

751. Ernst, E. The risk-benefit profile of commonly used herbal therapies: Ginkgo, St. John's Wort, Ginseng, Echinacea, Saw Palmetto, and Kava. Ann. Intern. Med. 2002, 136, 42-53. [CrossRef] [PubMed]

752. Shi, S.; Klotz, U. Drug interactions with herbal medicines. Clin. Pharmacokinet. 2012, 51, 77-104. [CrossRef] [PubMed]

753. Zeller, T.; Muenstedt, K.; Stoll, C.; Schweder, J.; Senf, B.; Ruckhaeberle, E.; Becker, S.; Serve, H.; Huebner, J. Potential interactions of complementary and alternative medicine with cancer therapy in outpatients with gynecological cancer in a comprehensive cancer center. J. Cancer Res. Clin. Oncol. 2013, 139, 357-365. [CrossRef]

754. Goey, A.K.; Beijnen, J.H.; Schellens, J.H. Herb-drug interactions in oncology. Clin. Pharmacol. Ther. 2014, 95, 354-355. [CrossRef]

755. Goey, A.K.; Mooiman, K.D.; Beijnen, J.H.; Schellens, J.H.; Meijerman, I. Relevance of in vitro and clinical data for predicting CYP3A4-mediated herb-drug interactions in cancer patients. Cancer Treat. Rev. 2013, 39, 773-783. [CrossRef]

756. Golden, E.B.; Lam, P.Y.; Kardosh, A.; Gaffney, K.J.; Cadenas, E.; Louie, S.G.; Petasis, N.A.; Chen, T.C.; Schönthal, A.H. Green tea polyphenols block the anticancer effects of bortezomib and other boronic acid-based proteasome inhibitors. Blood 2009, 113, 5927-5937. [CrossRef]

757. Dy, G.K.; Bekele, L.; Hanson, L.J.; Furth, A.; Mandrekar, S.; Sloan, J.A.; Adjei, A.A. Complementary and alternative medicine use by patients enrolled onto phase I clinical trials. J. Clin. Oncol. 2004, 22, 4810-4815. [CrossRef]

758. Sparreboom, A.; Cox, M.C.; Acharya, M.R.; Figg, W.D. Herbal remedies in the United States: Potential adverse interactions with anticancer agents. J. Clin. Oncol. 2004, 22, 2489-2503. [CrossRef] [PubMed]

759. Arslan, D.; Tural, D.; Akar, E. Herbal administration and interaction of cancer treatment. J. Palliat. Med. 2013, 16, 1466-1476. [CrossRef] [PubMed]

760. Brienza, R.S.; Stein, M.D.; Fagan, M.J. Delay in obtaining conventional healthcare by female internal medicine patients who use herbal therapies. J. Women's Health Gend. Based Med. 2002, 11, 79-87. [CrossRef] [PubMed]

761. Han, E.; Johnson, N.; DelaMelena, T.; Glissmeyer, M.; Steinbock, K. Alternative therapy used as primary treatment for breast cancer negatively impacts outcomes. Ann. Surg. Oncol. 2011, 18, 912-916. [CrossRef] [PubMed]

762. Johnson, S.B.; Park, H.S.; Gross, C.P.; Yu, J.B. Complementary Medicine, Refusal of Conventional Cancer Therapy, and Survival Among Patients With Curable Cancers. JAMA Oncol. 2018, 4, 1375-1381. [CrossRef] [PubMed]

763. Xiang, H.; Zhang, J.; Lin, C.; Zhang, L.; Liu, B.; Ouyang, L. Targeting autophagy-related protein kinases for potential therapeutic purpose. Acta Pharm. Sin. B 2020, 10, 569-581. [CrossRef]

764. Jaskulska, A.; Janecka, A.E.; Gach-Janczak, K. Thapsigargin-From Traditional Medicine to Anticancer Drug. Int. J. Mol. Sci. 2020, 22, 4. [CrossRef] 